\title{
Severe exacerbations and acute respiratory failure in COPD
}

Citation for published version (APA):

Sprooten, R. (2021). Severe exacerbations and acute respiratory failure in COPD. [Doctoral Thesis, Maastricht University]. ProefschriftMaken. https://doi.org/10.26481/dis.20211008rs

Document status and date:

Published: 01/01/2021

DOI:

$10.26481 /$ dis.20211008rs

Document Version:

Publisher's PDF, also known as Version of record

\section{Please check the document version of this publication:}

- A submitted manuscript is the version of the article upon submission and before peer-review. There can be important differences between the submitted version and the official published version of record.

People interested in the research are advised to contact the author for the final version of the publication, or visit the DOI to the publisher's website.

- The final author version and the galley proof are versions of the publication after peer review.

- The final published version features the final layout of the paper including the volume, issue and page numbers.

Link to publication

\footnotetext{
General rights rights.

- You may freely distribute the URL identifying the publication in the public portal. please follow below link for the End User Agreement:

www.umlib.nl/taverne-license

Take down policy

If you believe that this document breaches copyright please contact us at:

repository@maastrichtuniversity.nl

providing details and we will investigate your claim.
}

Copyright and moral rights for the publications made accessible in the public portal are retained by the authors and/or other copyright owners and it is a condition of accessing publications that users recognise and abide by the legal requirements associated with these

- Users may download and print one copy of any publication from the public portal for the purpose of private study or research.

- You may not further distribute the material or use it for any profit-making activity or commercial gain

If the publication is distributed under the terms of Article $25 \mathrm{fa}$ of the Dutch Copyright Act, indicated by the "Taverne" license above, 


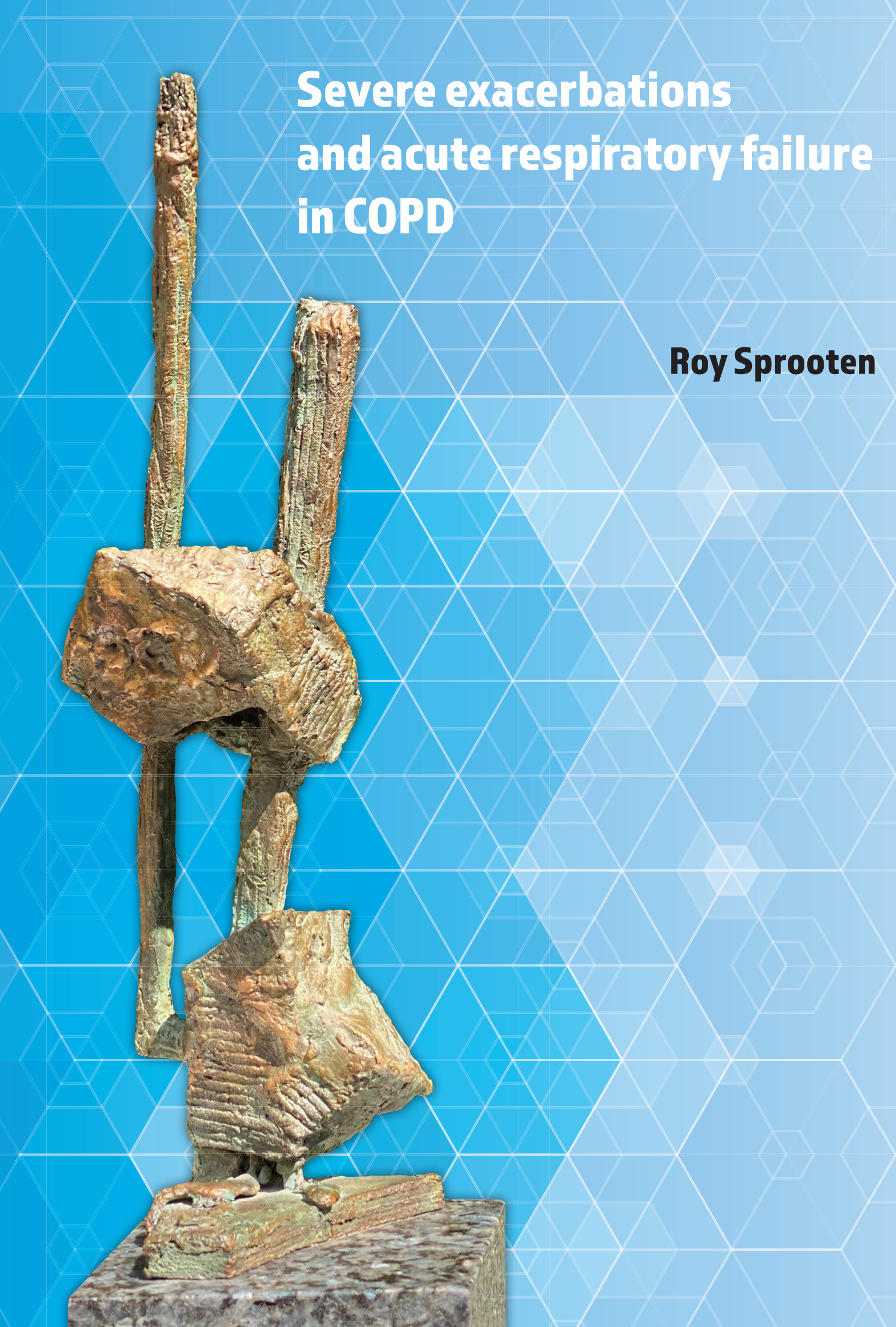



SEVERE EXACERBATIONS

AND

ACUTE RESPIRATORY FAILURE

IN COPD 
copyright $(C)$ Roy Sprooten 2021

All rights reserved. No part of this book may be reproduced or transmitted in any form or by any means without permission in writing by the author, or when appropriate, by the publishers of the publication.

Layout: Tiny Wouters

Omslagontwerp: Strategyminds

Omslagbeeld: "gebroken leven" (compositie in brons)

beeldhouwer Marcel Sprooten

Production: ProefschriftMaken.nl

ISBN: 978-94-6423-370-4 


\title{
SEVERE EXACERBATIONS AND
}

\section{ACUTE RESPIRATORY FAILURE IN COPD}

\author{
Proefschrift \\ ter verkrijging van de graad van doctor aan de Universiteit Maastricht, \\ op gezag van de Rector Magnificus, Prof. dr. Rianne M. Letschert, \\ volgens het besluit van het College van Decanen, \\ in het openbaar te verdedigen \\ op vrijdag 8 oktober 2021 om 12.00 uur
}

door

Roy Sprooten 


\section{Promotor}

Prof. dr. E.F.M. Wouters

\section{Copromotoren}

Prof. dr. F.M.E. Franssen

Prof. dr. G.G.U. Rohde

\section{Beoordelingscommissie}

Prof. dr. R.P. Koopmans (voorzitter)

Prof. dr. H.J.G.M. Crijns

Prof. dr. W. Janssens (Katholieke Universiteit Leuven, België)

Prof. dr. J. Verbraecken (Universitair Ziekenhuis Antwerpen, België)

Dr. D.C.J.J. Bergmans 


\section{Contents}

Chapter 1. General introduction

Chapter 2. Aims and outline of the thesis

Chapter 3. Long-term survival after admission for COPD exacerbation:

a comparison with the general population

Chapter 4. Predictors of 1-year mortality at hospital admission for acute exacerbations of chronic obstructive pulmonary disease

Chapter 5. A risk stratification for short-term mortality at hospital admission for acute exacerbation of COPD

Chapter 6. Predictors for short- and long-term mortality in COPD patients requiring non-invasive positive pressure ventilation for the treatment of acute respiratory failure

Chapter 7. Nocturnal non-invasive ventilation in COPD patients with prolonged hypercapnia after ventilatory support for acute respiratory failure: a randomised, controlled, parallel-group study.

Chapter 8. Increased small intestinal permeability during acute exacerbation of COPD

Chapter 9. Gut integrity in relation to exacerbations in patients with chronic 203 obstructive pulmonary disease

Chapter 10. General discussion 221

Summary 247

Samenvatting 253

Impact paragraph 261

Dankwoord

Curriculum vitae 277

List of publications 


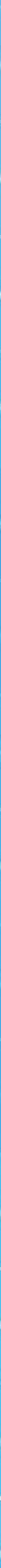




\section{Chapter 1}

General introduction 



\section{Definition and prevalence of COPD: Chronic Obstructive Pulmonary Disease}

Chronic obstructive pulmonary disease (COPD) is defined as a common, preventable and treatable disease that is characterized by persistent respiratory symptoms and airflow limitation due to airway and/or alveolar abnormalities which is the result of small airway remodelling and loss of elastic recoil due to alveolar destruction. ${ }^{1}$ To increase the awareness of diagnosing COPD, a fixed ratio Forced Expiratory Volume in one second $\left(\mathrm{FEV}_{1}\right) /$ Vital Capacity (VC) of 0.70 is generally used as the threshold to define COPD. ${ }^{1}$ COPD is usually assumed as a progressive disorder and associated with an enhanced chronic inflammatory response in the airways and the lungs to noxious particles or gases mostly cigarette smoke. ${ }^{1}$ Indeed, based on a limited set of spirometric data in young working men in London, Fletcher and Peto developed the concept of COPD as a self-inflicted condition caused by tobacco smoking with an accelerated decline of lung function in susceptible smokers. This classic concept of COPD dominated the scientific and clinical scene for almost half of a century. ${ }^{2}$ In the last decade there is upcoming evidence suggesting that risk factors other than smoking play a substantial role in the development of the pathophysiological impairment associated with the definition of COPD: outdoor air pollution, occupational exposure, respiratory infections in childhood, as well as low lung function at birth and childhood are contributing factors to reach the lung function criteria compatible with COPD. ${ }^{3,4}$ In particular, the lung function value reached in early adulthood is important with respect to the pathophysiological diagnosis of COPD later in life. In cohort studies, half of the persons presenting with airflow limitation followed the classic paradigm that implies a rapid decline in $\mathrm{FEV}_{1}$ from a normal level of lung function in early childhood, whereas the other half had a rather normal decline in $\mathrm{FEV}_{1}$ but started from a low initial value of $\mathrm{FEV}_{1}{ }^{4}$ Others confirm that low peak lung function in early adulthood is common in the general population and that trans-generational effects contribute to this early childhood condition. ${ }^{5}$ Airflow limitation is not the only pathophysiologic trait in COPD: airways disease as well as parenchymal destruction or emphysema are considered part of the structural abnormalities in COPD. ${ }^{1}$ Physiomics of lung function involvement in COPD patients clearly demonstrate that this condition is even more heterogeneous than airflow limitation and emphysema only. ${ }^{6}$

Despite this growing understanding in the pathophysiological impairment of COPD, for many years grading of COPD (stage 1 to 4 ) was only based on the values of postbronchodilator $\mathrm{FEV}_{1}{ }^{7}{ }^{7}$ In the most recent revision, assessment of symptoms and risk of exacerbations or hospitalisations are introduced in the combined assessment of COPD 
(Figure 1.1). ${ }^{8}$ The first GOLD 2001 report introduced a new pragmatic classification of COPD severity based on spirometry and arterial blood gas disturbances. ${ }^{9}$ In 2006 the classification was more specified. For the first time it was noted that COPD was also characterised by significant extra-pulmonary manifestations and relevant comorbidities. ${ }^{9}$ In 2011 there was a general consensus that besides FEV 1 other characteristics contribute to the burden of the disease. Multidimensional indices as BODE (body mass index, airflow Obstruction, Dyspnoea, and Exercise capacity) ${ }^{10}$ or ADO (Age, Dyspnoea, and airflow Obstruction) ${ }^{11}$ demonstrated better prediction in outcome than $\mathrm{FEV}_{1}$ only. Later on, the frequent exacerbator phenotype was broadly accepted and used to create the $A B C D$ classification square. ${ }^{12}$ This classification highlighted two features of COPD: symptoms and exacerbations and was aimed to be helpful in the management of COPD. In 2017 the refined ABCD classification was developed. The spirometric grades were separated from the "old" four quadrants and the treatment recommendations were based on symptoms and exacerbation history. ${ }^{9}$ This provides a rough guide in tailoring pharmacological treatment. Nevertheless, a prospective cohort study by Han et al showed that the exacerbation status of most individuals might vary markedly from year to year. Only $2 \%$ of the frequent exacerbators had consistently two or more exacerbations per year. ${ }^{13}$ This instability in exacerbation of COPD (ECOPD) frequency in patient's trajectory limits the clinical use of the threshold of two or more ECOPD in prior year in the refined ABCD classification for COPD. ${ }^{13}$ The use of different scales and non-fitting cut-off points, also result in misclassification of one-third of clinically treated COPD patients with a direct impact on the disease management approach. $^{14}$ In particular, the choice of the symptom measure impacts COPD classification more than the choice of the exacerbation assessment. ${ }^{15}$

The lack of a gold standard to make the diagnosis of COPD can be illustrated by recent findings of the impact of early abnormalities in those subjects without airflow limitation. Tan et al reported exacerbation-like events in half the frequency in individuals without COPD compared with COPD patients. ${ }^{16}$ In a follow-up study of 8246 participants of the COPD Gene study, Bowler et al reported that acute episodes of respiratory disease are common in current and former smokers without COPD, particularly when there is a history of prior events and poor respiratory health status. ${ }^{17}$ Others confirmed these findings: respiratory symptoms and even exacerbations are common in current and former smokers with preserved lung function. ${ }^{18}$ 
Figure 1.1 GOLD classification.

\section{7: The refined $A B C D$ assessment tool}

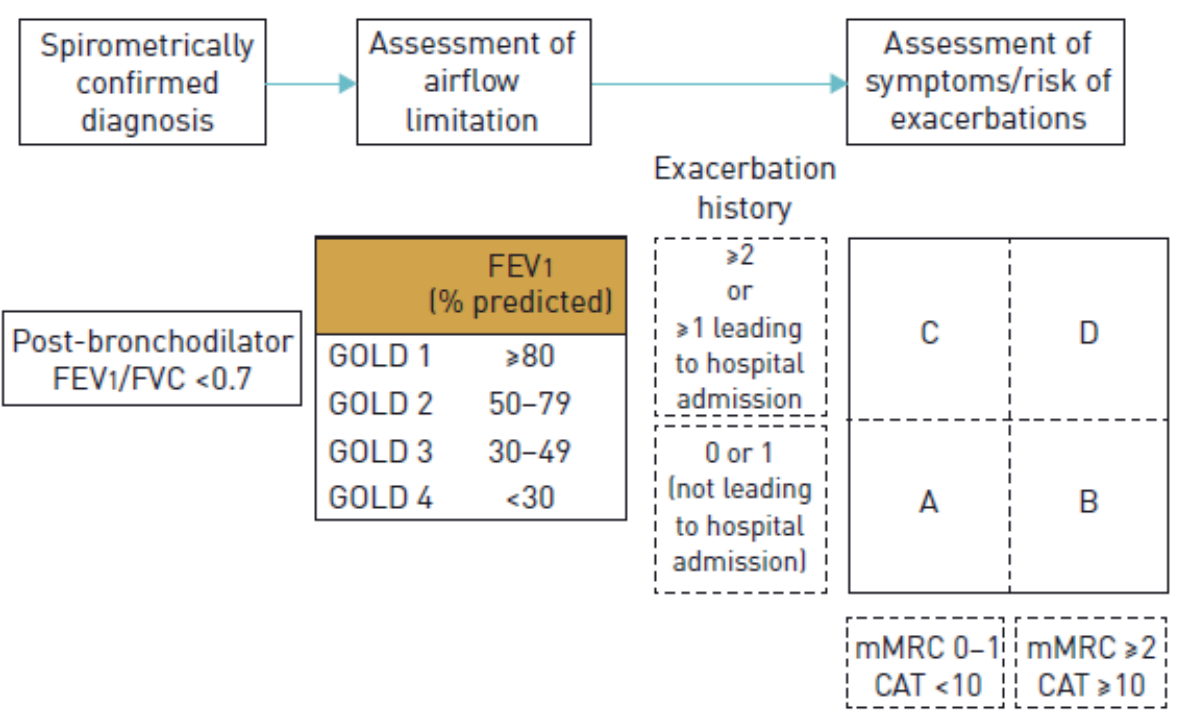

Symptoms

Reference: Rodriguez-Roisin, R., et al, Global Initiative for Chronic Obstructive Lung Disease (GOLD) 20th Anniversary: a brief history of time. European Respiratory Journal, $2017 .^{9}$

Chest Computed Tomography (CT) is a new, widely available non-invasive imaging modality that can provide insight into the structural and pathophysiological pulmonary parameters. In recent years, large observational studies as COPD Gene and CanCOLD significantly contributed to a better understanding of respiratory-related impairments in smokers with normal spirometry. ${ }^{19}$ In so-called GOLD 0 patients (absence of airflow limitation), more than $40 \%$ had CT evidence of emphysema or airway thickening. ${ }^{20}$ These data were confirmed in the Canadian cohort: respiratory bronchiolitis as well as air trapping was more frequent in ever-smokers with normal lung function than in mild or moderate COPD patients. Bronchial wall thickening was found in more than half of smokers without COPD as well as the proportion of individuals with emphysema was elevated in ever-smokers with normal lung function (30\%) versus the normal population (11\%). Intriguingly, this presence of emphysema on CT was associated with chronic cough and phlegm production, wheeze, dyspnoea, impaired health status and even increased risk of more than two exacerbations over 12 months. ${ }^{21}$ In a cohort of symptomatic current or former smokers with preserved pulmonary function, Woodruff 
et al reported a greater airway wall thickening in these current and former smokers without differences in emphysematous features compared to those with COPD related symptoms. ${ }^{18,22}$ All these data clearly illustrate that CT has proven instrumental in identifying structural abnormalities even in smokers with preserved lung function.

Globally, COPD is a high prevalent disease. Despite all efforts of the World Health Organization to reduce the impact of COPD, the prevalence of COPD is high and expected to increase the next years. ${ }^{23}$ Based on a meta-regression epidemiological model of population based studies providing spirometry-based prevalence rates of COPD worldwide from January 1990 to December $2014^{23}$, the authors estimated in 1990 about 227.3 million COPD cases corresponding to a global prevalence of $10.7 \%$. In 2010 these figures increase to 384 million COPD cases with a worldwide prevalence of $11.7 \%$ or an absolute increase of $68.9 \% .^{23}$ In 2019 , Varmaghani published a metaanalysis based on population-based studies performed across the world reported between January 2004 and May 2015. Based on sixty published studies, they calculated a global COPD prevalence of $12.16 \%{ }^{24}$ These results represent a higher global prevalence of COPD compared to previous studies. ${ }^{23}$ The highest prevalence was found in the Region of the Americas (13.3\% in 1990 and 15.2\% in 2010) and the lowest was calculated in the South East-Asia Region (7.9\% in 1990 and 9.7\% in 2010). ${ }^{23,24}$ The percentage increase in COPD subjects was the highest in the Eastern Mediterranean region followed by the African region. The lowest percentage increase of COPD cases $(22.5 \%)$ was estimated in the European region. ${ }^{23}$ This difference of prevalence per region could be partially explained by factors such as level of industrialization, prevalence of smoking and amount of exposure of noxious particles, geographic situation and difference in culture. The difference across countries in approach and in clinical use of the definition and assessment of COPD could be another explanation for the variety in prevalence of COPD across countries. ${ }^{1,25,26} \mathrm{~A}$ worldwide conducted study, measuring the prevalence of spirometrically confirmed COPD according the GOLD definition in 2006, reported a prevalence of stage II $\left(\mathrm{FEV}_{1}<80 \%\right)$ or higher stages of COPD of $10.1 \%$, for men $11.8 \%$ and for women $8.5 \%{ }^{27} \mathrm{~A}$ recent meta-analysis found that more than $12 \%$ of the general population globally suffered from COPD. The authors of this study estimated that of all COPD patients of the world $44.16 \%$ had mild (stage I: $\mathrm{FEV}_{1} / \mathrm{FVC}<70 \%$ and $\mathrm{FEV}_{1} \geq 80 \%$ predicted) COPD, $44.22 \%$ had moderate (stage II: $\mathrm{FEV}_{1} / \mathrm{FVC}<70 \%$ and $50.0 \% \leq \mathrm{FEV}_{1}<80 \%$ predicted) COPD and the remaining patients suffered from severe (stage III: $\mathrm{FEV}_{1} / \mathrm{FVC}<70 \%$ and $30 \% \leq \mathrm{FEV}_{1}<50 \%$ predicted; and very severe COPD or stage IV: $\mathrm{FEV}_{1} / \mathrm{FVC}<70 \%$ and $\mathrm{FEV}_{1}<30 \%$ predicted) COPD. ${ }^{24}$ There figures about prevalence of COPD and prevalence of higher stages of COPD were consistently higher than reported in previous studies. 
In most European countries exact figures of the prevalence of spirometry-defined COPD are lacking. With the available data the global prevalence of COPD in Europe is estimated to be $12.38 \% .{ }^{28}$ In the Netherlands a $4 \%$ prevalence of COPD in the general population has been estimated. ${ }^{28}$ Others reported very different data. A study performed in the general population of Maastricht reported a prevalence of $24 \%$ in inhabitants aged 40 years or over. ${ }^{29}$ Another study performed in Rotterdam reported a prevalence of $12 \% .{ }^{30}$ In absolute numbers, in $2018 .{ }^{31} 613.800$ COPD patients were diagnosed by general practitioners in the Netherlands; 304.800 men (35.6 per 1000) and 309.000 women (35.6 per 1000). The incidence in 2018 was 27.100 new cases: 4.300 (0.5 per 1000) men with chronic bronchitis and 13.400 (1.6 per 1000) cases with emphysema/COPD and 5.900 (0.7 per 1000) and 13.700 (1.6 per 1000) in women. ${ }^{31}$ During the period 2011-2018 the prevalence of COPD (reported by general practitioners) slightly decreased in the Netherlands. These discrepancies in prevalence rates are largely explainable by differences in the definition of COPD and the lack of a gold standard to really estimate prevalence of this disease condition.

\section{Personal burden}

The impact on patient's life and health status due to COPD is tremendous. Health status which is defined as the impact of health on a person's ability to perform and derive fulfilment from activities of daily life, seems to be strongly related to disease burden. COPD is a devastating disease and interrupts daily activity, sleep pattern and the ability to exercise. In a survey performed in Australia in the period 2017- 2018, self-reported health status in COPD patients was worse rated compared to people without COPD: $20 \%$ of patients with COPD aged 45 years or over reported their health status as poor, compared with $5.4 \%$ in subjects without COPD of the same age. Health status was reported as very good and excellent in respectively $17 \%$ and $4.9 \%$ of COPD patients, while it was $34 \%$ and $17 \%$ in the non-COPD group. The same pattern is found regarding the psychological distress experience by people aged 45 years and over with and without COPD. In $19 \%$ of the patients with COPD the psychological distress was rated as high and in $17 \%$ very high compared to $8.3 \%$ and $4.0 \%$ in the non COPD subjects. ${ }^{32}$

The personal burden significantly increases from primary to tertiary care but even in primary care more than $68 \%$ of the COPD patients were highly symptomatic based on COPD Assessment Test (CAT) score: $8 \%$ of them had a high frequency of exacerbations of the disease (29). In the secondary care setting $90 \%$ of patients scaled the burden as highly symptomatic with reduced functional mobility. These data reveal the high 
symptom and disease burden despite standard pharmacological treatment and underscore the need to more tailored treatment independent of the healthcare setting. ${ }^{33}$

Caregivers play a pivotal role in care for COPD patients. They provide the majority of caring at home like helping in household chores, personal care, emotional support or management in treatments or healthcare appointments. ${ }^{34-36}$ COPD patients often reported that their disease affects their family life and has a negative impact on the relationship between caregiver and patient. The other way around, caregivers heath related quality of life can affect the quality of life of the COPD patient. ${ }^{37}$ In particular, the occurrence of COPD exacerbations (ECOPD) and hospitalisations of ECOPD and life threatening events needing mechanical ventilation enhance the caregiver's burden. ${ }^{38}$ Fortunately, despite the huge distress caregivers experience also positive caring appraisal and consider this sacrifice as an opportunity for personal growth. ${ }^{39}$

\section{Health economic burden}

Besides the tremendous personal and social burden of COPD, the economic impact of this disease is enormous and expects to increase in the following years. According to the Global Burden of Disease Study, COPD is ranked as sixth leading cause of global burden of disease in $2017 .{ }^{40}$ The economic burden caused by COPD to the health care system is expected to increase over the time. In the United States the National Heart, Lung and Blood Institute assessed the annual costs of COPD in 2010 at 49.9 billion USD and within direct costs estimated at 29.5 billion USD, which will increase to 49.0 billion USD by $2020 .^{41}$ The indirect morbidity costs (loss productivity because of illness) were estimated at 8 billion USD and the indirect mortality costs (loss of productivity because of early death) were figured at 12.4 billion USD. ${ }^{42,43}$ In Australia COPD was ranked as the third leading specific cause of total disease burden, accounting for huge health care costs. In this continent, the health care costs involving COPD care is estimated 976.9 million USD which represents $24 \%$ of the respiratory diseases expenditure and $0.8 \%$ of total disease expenditure. ${ }^{44}$

Based on European estimates, $6 \%$ of the total healthcare budget is allocated to the respiratory diseases in 2011: 56\% of this budget accounts for COPD. The total costs of respiratory diseases every year is figured at a minimum of 380 billion euros and the estimated annual economic burden of COPD including direct and indirect costs is amounted at 48.4 billion euros (Table 1.1). ${ }^{45}$ In the European region COPD accounts for 
the greatest economic burden of respiratory diseases on healthcare and loss of productivity. ${ }^{45}$

Table 1.1 Aggregated annual direct and indirect costs and the value of disability-adjusted life-years (DALY's) lost for EU countries 2011 by disease (billions of euro at 2011 values).

\begin{tabular}{lcccc}
\hline & $\begin{array}{c}\text { Direct costs } \\
\text { (euro bn) }\end{array}$ & $\begin{array}{c}\text { Indirect costs" } \\
\text { (euro bn) }\end{array}$ & $\begin{array}{c}\text { Monetised value os DALY's } \\
\text { lost (euro bn) }\end{array}$ & $\begin{array}{c}\text { Total costs } \\
\text { (euro bn) }\end{array}$ \\
\hline COPD & 23.3 & 25.1 & 93.0 & 141.4 \\
Asthma & 19.5 & 14.4 & 38.3 & 72.2 \\
Lung cancer & 3.35 & $\mathrm{NA}$ & 103.0 & 106.4 \\
TB & $0.54^{\#}$ & $\#$ & 5.37 & 5.9 \\
OSAS & 5.2 & 1.9 & $\mathrm{Na}$ & 7.1 \\
Cystic fibrosis & 0.6 & $\mathrm{NA}$ & $\mathrm{Na}$ & 0.6 \\
Pneumoniae/ALRI & 2.5 & $\mathrm{NA}$ & 43.5 & 46.0 \\
Total & 55.0 & 41.4 & 283.2 & 379.6 \\
\hline
\end{tabular}

COPD: chronic obstructive pulmonary disease; TB: tuberculosis; OSAS: obstructive sleep apnoea syndrome; ALRI: acute lower respiratory infections; NA: not available. \# primary care, hospital outpatient and inpatient care, drugs and oxygen; ๆ lost production including work absence and early retirement. ${ }^{+}$indirect cost included with direct costs. Reference: European Lung White Book, $2013 .^{45}$

In the Netherlands the healthcare costs for COPD care during the period 2007 to 2017 increased from 415 million to 912 million euro. These tremendous COPD care costs are $1 \%$ of the total health care expenditure. From the total expenditure of respiratory diseases related budget $31 \%$ is directed to COPD care. ${ }^{31}$ of the COPD related health care costs $32 \%$ represents the expenditure for the elderly, $24 \%$ for hospital care and $21 \%$ medical prescription. Of interest, the highest health care expenditure for COPD was taken by subjects aged 65 years and over (Figure 1.2). ${ }^{31}$

Costs related to COPD exacerbations, hospitalizations and related comorbidities impose a tremendous burden on health care systems. ${ }^{26} \mathrm{~A}$ recently published systematic literature study, reviewed the economic burden in USA, Asia and Europe and identified key cost drivers in management of COPD patients. ${ }^{46}$ They found in the USA the annual per patient direct medical costs in 20094273 USD and in 201411.232 USD. The amount of direct medical costs is related to the frequency of exacerbation of COPD (ECOPD). In patients with no ECOPD the annual per patient direct costs were 1425 USD, whereas in patients suffering from two or more ECOPD these costs increased to 12.765 USD. The hospitalisation costs for severe COPD exacerbation was nearly three times higher compared to moderate COPD exacerbation. The same pattern was seen in Asia and Europe. The authors also identified different factors affecting the costs of COPD management including severity of disease frequency of exacerbations and hospital admissions. ${ }^{46}$ Exacerbations of COPD (ECOPD) and hospitalizations for ECOPD represent 
a pivotal healthcare problem in the Netherlands as direct costs of COPD in the Netherlands are for over $55 \%$ driven by emergency visits and hospitalizations related to acute exacerbations. ${ }^{47}$ Besides exacerbations, the personal and economic impact is also related to the severity of the disease and symptoms. ${ }^{48}$ Patients with the worse degree of COPD and a large number of comorbidities represent the highest costs in COPD care. $^{48-51}$

Figure 1.2 COPD related healthcare expenditure in 2017 in the Netherlands.

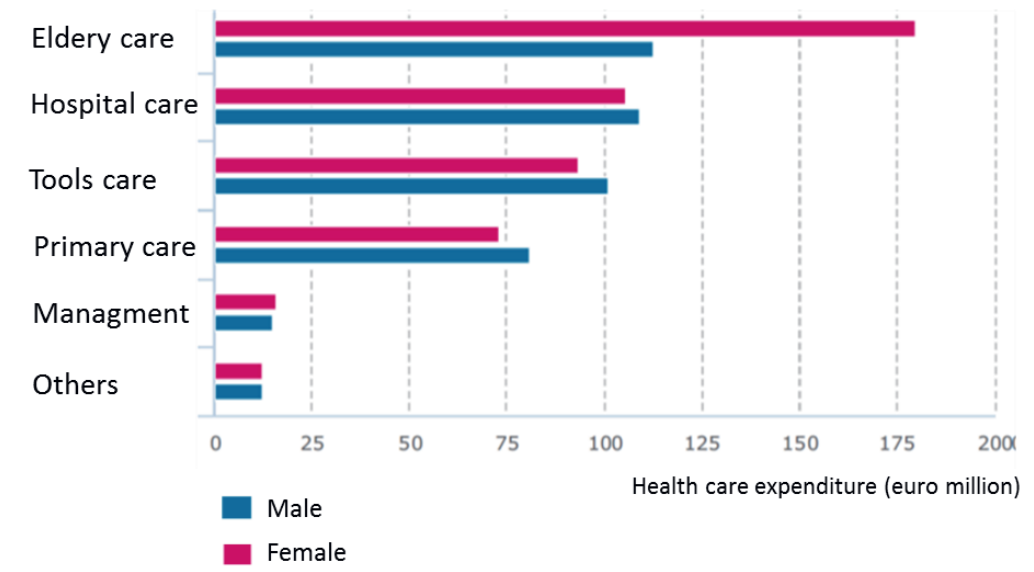

Reference: RIVM https://www.volksgezondheidenzorg.info/onderwerp/copd/kosten. ${ }^{31}$

Loss of productivity due to COPD accounts for $50 \%$ of the overall COPD related societal cost in the Netherlands. ${ }^{52}$ Regarding the indirect costs $6-52 \%$ of patients with COPD has not the ability to maintain a job due to the impairing symptoms of COPD. ${ }^{53}$ Accordingly the average costs due to COPD related absenteeism of the employer were estimated at 1900 euro per year per employee, worsening with increasing age. ${ }^{54}$ In a study in which COPD-related productivity loss was studied, nearly $20 \%$ reported that they were completely impaired to work due to COPD, and $10 \%$ were limited in their work in the previous year. $^{50}$

\section{Social burden}

The Disability Adjusted Life Years (DALYs), combining the loss of quantity of life as well as loss of quality of life, can be used as an indicator for the social burden of COPD. In 
2015, worldwide the DALY's loss due to COPD were estimated at 63.9 billion, which represented $2.6 \%$ of the total global burden of disease and therefore ranking as eighth among the 315 global burden disease causes. ${ }^{40}$ In 2015 in the Netherlands COPD was ranked with 188.900 DALY's as fourth in the top ten list of diseases with the highest DALY's. Only coronary heart disease, cerebrovascular disease and diabetes mellitus are accounting for more DALY's each. In men the DALY's for COPD were 89.900 and for women 92.600, ranking fourth and fifth respectively. Per Dutch COPD patient a mean DALYs of 5.6 was assessed. ${ }^{31,55}$

\section{Mortality}

COPD as life-threatening progressive disease is associated with a high mortality throughout the world. ${ }^{26}$ In 2016 worldwide COPD was the third leading cause of death after ischemic heart disease and stroke ${ }^{56-59}$ : worldwide, 3.041 .000 deaths or $5,7 \%$ of total deaths are related to COPD. ${ }^{59-61}$ In the United States COPD, including emphysema and chronic bronchitis is the fourth leading cause of death. ${ }^{62,63}$ The average number, unadjusted and age adjusted mortality rates per 100.000 persons of self-reported, physician-diagnosed COPD among adults of all ages in the United States were respectively 146.412, 45.7 and 39.7. In Australia, COPD is the fifth leading cause of death after respectively coronary heart disease, dementia and Alzheimer disease, cerebrovascular disease and lung cancer. ${ }^{44}$ Interestingly, based on the mortality data from the Australian Institute for Health and Welfare among men aged over 45 years the mortality rates decreased enormously by two thirds between 1980 and 2010 . Otherwise, the mortality rate among women increased almost three times in the period 1965 to 1996 and is now flattening or even declining in the period 1996 and $2010 .^{44}$ In the Netherlands 6.879 patients diagnosed with COPD died in 2018 of whom 3.442 men (40.2 per 100.000) and 3.437 women (39.6 per 100.000) (Figure 1.3). ${ }^{31}$ The death rate due to COPD in males is decreasing from the beginning of this century. In women the mortality rate increased in the period 1980-1999, and flattened afterwards (Figure 1.3). . $^{31}$

ECOPD have a major negative impact on mortality of this disease. Different studies reported a wide range of mortality rates of hospitalised-ECOPD (H-ECOPD). The inhospital mortality varies between $2.9-10.4 \%$, 90-day mortality between $4.8-13.9 \%$ and 1-year mortality between $23.0-27.7 \%{ }^{64-68}$ 
Figure 1.3 Trend of death rate COPD 1980-2018 in the Netherlands.

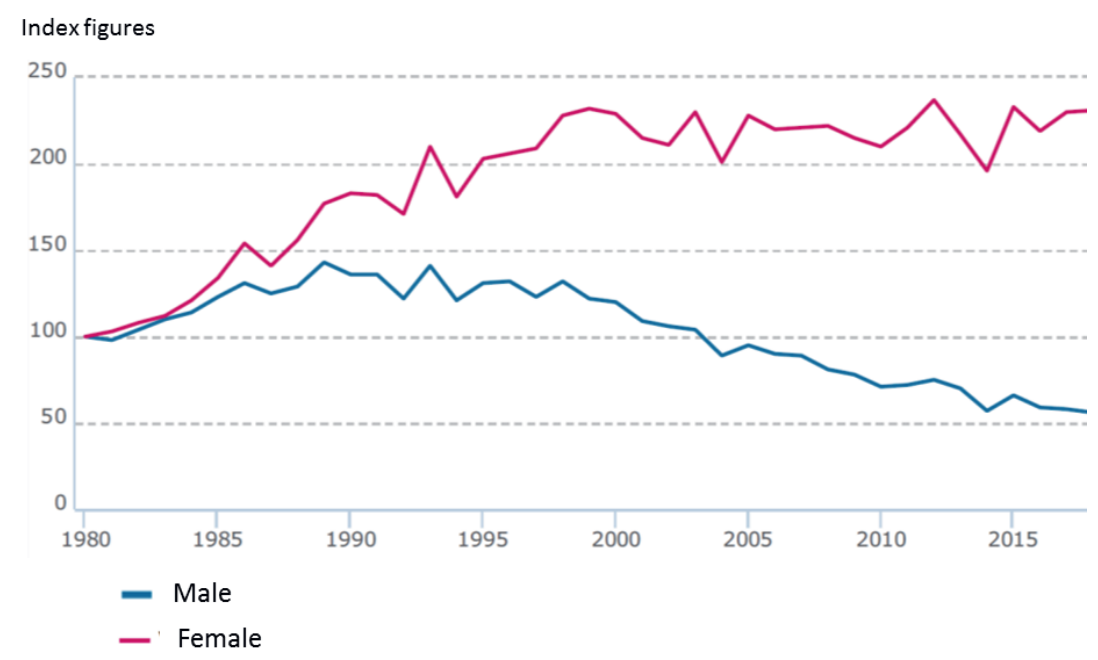

Reference: RIVM, www.volksgezondheidenzorg.info/onderwerp/copd. ${ }^{31}$

\section{Management of COPD: reduce symptoms and risks}

The clinical manifestations of COPD include chronic cough, breathlessness accompanied with impaired exercise tolerance and /or an abnormal and chronic sputum production, which are progressive and can vary from day-to-day. ${ }^{1,69}$ Accordingly, performing daily activities in COPD patients is often challenging ${ }^{70}$, which can lead to a substantial reduction in quality of life. ${ }^{71,72}$ Pharmacological therapy aims to reduce COPD symptoms, reduce the frequency and severity of exacerbations, and improve health status and exercise tolerance. ${ }^{8}$ Symptomatic relief is largely approached by bronchodilator therapy. These medications act by altering airway smooth muscle tone and the improvements in expiratory flow reflect widening of the airways rather than changes in lung elastic recoil. ${ }^{8}$ Two main classes of bronchodilators are used in clinical practice: beta- 2 agonists and anti-cholinergics. The principal action of beta- 2 agonists is to relax airway smooth muscle by stimulating beta-2 receptors, which increases cyclic adenosine monophosphate (CAMP) and produces functional antagonism to bronchoconstriction. A broad set of short-acting beta-2 agonists (SABA) and long-acting beta-2 agonists (LABA) is currently available. Anti-muscarinic drugs block the bronchoconstrictor effects of acetylcholine on $\mathrm{M} 3$ muscarinic receptors expressed in airway smooth muscle. ${ }^{73}$ Short-acting anti-muscarinics (SAMA) also block the inhibitory 
neuronal receptor $\mathrm{M} 2$, which potentially can cause vagally induced bronchoconstriction. $^{74}$ Long-acting muscarinic antagonists (LAMA) have prolonged binding to M3 muscarinic receptors with prolonged duration of the bronchodilator effects. ${ }^{73}$ Bronchodilators with different mechanisms and durations of action are frequently combined to increase the degree of bronchodilation and to lower risk of side-effects. Numerous combinations of a LABA and LAMA in a single inhaler are now available on the market. Alleviation of symptoms such as dyspnoea and cough in moderate to severe COPD can be managed by long acting bronchodilators including LABA and LAMA. A systematic review showed a positive trend in improvement of symptoms and greater quality of life scores in patients using LABA/LAMA combination compared to mono-therapy. The LAMA containing inhalers might be more effective to prevent exacerbations above inhalers without LAMA. Furthermore analysis of the pooled data revealed that usage of inhaled corticosteroids (ICS) containing inhalers is associated with an increased risk of pneumonia. ${ }^{75}$

Based on a huge set of randomized clinical trials, recommendations and guidelines conclude now that LABAs as well as LAMAs significantly improve lung function, dyspnoea and health status but remaining symptoms are in most patients still very prominent and disabling. ${ }^{8}$ LABAs, LAMAs and LABA/LAMA combinations are also studied to reduce exacerbations in COPD patients. A systematic review and network meta-analysis conducted in 2018 showed that the LABA/LAMA combination was the most effective to reduce COPD exacerbations in the moderate to severe COPD group. The LAMA followed hereafter and LABA solely had the worse ranking. ${ }^{75}$ The same pattern was found for the outcome severe exacerbations. ${ }^{76}$ Based on these results it is generally assumed that LAMAs have a greater effect on exacerbation reduction compared with LABAs and that combination treatment with a LABA/LAMA reduces exacerbations compared with monotherapy.

Exacerbations are the main clinically relevant end-point for efficacy assessment of drugs with anti-inflammatory effects. ICS are extensively studied in COPD patients. Although classified as anti-inflammatory drugs, the direct effects of ICS on inflammation in COPD are still poorly documented. Systemic inflammation is one of the main characteristics of COPD and supports the reasoning for use of corticosteroids in the treatment of COPD. ICS are assumed to have effects on the inflammatory process in the bronchi of COPD patients, which is dominated by neutrophilic infiltration as well as by macrophage and CD8-positive T-lymphocytes. ${ }^{77}$ These cells stimulate a cascade of reactive oxygen particles, chemokines, cytokines and proteases. This chemical attack awakens and stimulates the inflammatory state and eventually causes tissue damage. 
Mechanistically, the corticosteroids bind to the intracellular glucocorticoid receptors, which regulate the gene expression through glucocorticoid response elements. Barnes et al showed that these elements do not directly cause the anti-inflammatory effects of the corticosteroids but that the regulation of the histone acetylation plays a pivotal role in ICS intervention. ${ }^{78}$ Corticosteroids inhibit histone acetyltransferase (HAT) activity and accordingly promote the activity of histone deacetylase class 2 (HADAC2). HADAC2 is an enzyme that catalyse the removal of acetyl functional group from lysine residues of both histone and non-histone proteins and is accordingly a key molecule in the inhibition of production pro-inflammatory agents. ${ }^{79}$ The total HADAC activity in patients with COPD is significantly reduced. ${ }^{80}$ This observation may explain the relative steroid resistance in COPD.

Not surprisingly, most studies have found that regular treatment with ICS alone has limited effects on exacerbations. Studies in the 1990s of ICS monotherapy in COPD showed that this anti-inflammatory therapy reduced the bronchial inflammation but had varying effects on lung function measurements such as $\mathrm{FEV}_{1}$ and peak expiratory flow (PEF). In short-term and long term (3 years) randomized placebo controlled studies of ICS in COPD patients no benefit on pulmonary lung function was found: these studies failed to show any lowering on the rate of decline in pulmonary lung function and in addition ICS therapy not changed the $\mathrm{FEV}_{1}$ beneficially in patient with moderate-severe COPD. ${ }^{81,82}$ Recently a systematic review study of randomized controlled trials of inhaled corticosteroid mono-therapy in stable COPD patients concluded that the use of inhaled corticosteroids has beneficial effects on frequency of exacerbations and on the rate of decline of quality of life but in the absence of consistent effects on lung function decline or on mortality. ${ }^{83}$

Combining the ICS and LABA in one inhaler, a two-way synergistic activity between these components is assumed possible leading to improved outcomes. Indeed, the cellular action of ICS to translocate glucocorticoid receptors from the cytoplasm to the nucleus is enhanced in the presence of beta-agonists possibly causing an antiinflammatory effect greater than by ICS alone. Additionally, the ICS components stimulate the beta-receptor genes to produce more beta-receptors, leading to a greater bronchodilator effect of the LABA. ${ }^{84}$. Most studies combining ICS with a LABA are indeed more effective than the individual components to reduce exacerbations. The Towards a Revolution in COPD Health (TORCH) trial demonstrated in the ICS/LABA group a significant exacerbation reduction by $25 \%$ compared to placebo. In addition, they found a reduction of $17 \%$ in the risk of death in the ICS/LABA group versus placebo. Studies comparing ICS/LABA vs LABA alone revealed that the combination 
therapy reduces the exacerbation rate with $24 \%{ }^{85}$ Comparing the combination ICS/LABA vs ICS monotherapy showed a significant reduction of $13 \%$ in rate of exacerbations in favour for the combination therapy. In addition, in the ICS/LABA group the odds ratios of death were significantly lower. Several clinical studies compared the combination therapy ICS/LABA vs LAMA. In the INSPIRE study (Investigating New Standards for prophylaxis in Reducing Exacerbations) the efficacy of SalmeterolFluticasone (LABA/ICS) is compared to LAMA mono-therapy in preventing exacerbations in severe to very severe COPD patients. Between the groups there was no significant difference in exacerbation rate. In the LABA/ICS group a better health status was found as well as a lower withdrawal rate. ${ }^{85,86}$ More recently, the FLAME study (EFfect of Indacaterol Glycopyronium vs Fluticasone Salmeterol on COPD Exacerbations), investigated the comparative efficacy of LAMA/LABA (dual bronchodilators) Indacaterol-Glycopyrronium vs ICS/LABA Fluticasone-Salmeterol in reducing the risk of exacerbations COPD. This study demonstrated that for patients who had one or more exacerbations COPD in previous year the annual rate of moderate to severe exacerbations of COPD was significantly lower in the LABA/LAMA group (0.98) compared to the ICS/LABA group $(1.19, \mathrm{p}<0.001) .^{85}$

Currently, triple therapy of ICS/LAMA/LABA is studied compared to ICS/LABA, LABA/LAMA or LAMA monotherapy on lung function, health status and risk reduction of exacerbations. Several studies demonstrate that the triple therapy in a single inhaler is more effective in improvement of lung function and health status and especially in reducing the rate of exacerbations compared to other interventions. Additionally, usage of triple therapy has no greater risk of adverse events compared to ICS/LABA or LAMA monotherapy. ${ }^{87,88}$ Although mortality was not an endpoint in these studies a reduced mortality up to $42 \%$ in the single inhaler triple therapy was reported. ${ }^{89}$

A number of recent post-hoc studies have shown that blood eosinophil counts predict the magnitude of the effect of ICS in preventing future exacerbations. ${ }^{88,90-94}$ Cheng et al demonstrated a dose response effectiveness of ICS in COPD patients with elevated eosinophils and debated that eosinophil levels may help clinicians in individualizing treatment during exacerbation of COPD. ${ }^{95}$ Others confirm these findings. ${ }^{90}$ Hence, GOLD recommendations advise to use blood eosinophil counts as a marker for prediction the treatment effect of ICS on exacerbation risk. $^{8}$ Such approach may helpful for the clinician to balance the favourable effects of ICS against the risk of pneumonia complicating ICS therapy. ${ }^{83,96,97}$ In summary, initiating ICS treatment must be well considered as a potentially beneficial treatment in a subgroup of COPD patients (Table 1.2). ${ }^{98}$ 
Table 1.2 Factors to consider when initiating inhaled corticosteroids (ICS) treatment in combination with one or two long-acting bronchodilators in COPD patients.

\begin{tabular}{|c|c|c|}
\hline Strong support & Consider use & Avoid use \\
\hline $\begin{array}{c}\text { History of hospitalisation(s) for exacerbations of COPD\# } \\
\begin{array}{c}\text { 2 moderate exacerbations of COPD per year } \\
\text { Blood eosinophils }>300 \text { cells } \mu \mathrm{L}^{-1} \\
\text { History of, or concomitant, asthma }\end{array}\end{array}$ & $\begin{array}{c}1 \text { moderate exacerbation of COPD per year\# } \\
\text { Blood eosinophils } 100-300 \text { cells } \mu \mathrm{L}^{-1}\end{array}$ & $\begin{array}{c}\text { Repeated pneumonia events } \\
\text { Blood eosinophils }<100 \text { cells. } \mu \mathrm{L}^{-1} \\
\text { History of mycobacterial infection }\end{array}$ \\
\hline
\end{tabular}

\#: despite appropriate long-acting bronchodilator maintenance therapy.

Reference: Agusti A, Fabbri LM, Singh D, et al, Inhaled corticosteroids in COPD: friend or foe? European Respiratory Journal, 2018. ${ }^{98}$

Phosphodiesterase-4 (PDE-4) inhibitors form an intriguing new class of antiinflammatory drugs in COPD management. The principal action of PDE-4 inhibitors is to reduce inflammation by inhibiting the breakdown of intracellular CAMP. ${ }^{99,100}$ The PDE-4 inhibitor, roflumilast, has marked anti-inflammatory effects in COPD. ${ }^{100}$ PDE-4 inhibition is probably the only intervention now available to reduce moderate as well as severe exacerbations. Oral PDE-4 administration can have more adverse effects than inhaled medications, which are mostly gastro-intestinal symptoms. In an add-on therapy of roflumilast in COPD patients with uncontrolled disease, a significant reduction of moderate to severe COPD exacerbation was achieved in COPD patients when adding roflumilast on top of ICS/LABA or ICS/LABA/LAMA treatment regimens. ${ }^{101,102}$ Some studies claim anti-inflammatory effects by regular use of some antibiotics, azithromycin or erythromycin particularly. Macrolides have antibiotic and anti-inflammatory properties but its primary mode of action is attributed to its anti-inflammatory effects. Clinical improvement has been reported independent of the presence or absence of chronic airway infection and with antibiotic levels below the minimum inhibitory concentrations of several pathogenic bacteria. ${ }^{103}$ Following admission for a severe ECOPD, patients are likely to have heightened airway and systemic inflammation which may be attenuated by macrolide therapy. Macrolides are also pro-kinetic to the gastrointestinal (GI) tract and have been shown to reduce gastro-esophageal reflux, a major contributor to ECOPD. ${ }^{104}$

There is consistent evidence that prophylactic macrolide therapy reduces exacerbations across a spectrum of chronic airway diseases including COPD. Albert et al, in a randomized placebo-controlled trial, involving $>1100$ patients demonstrated a $30 \%$ reduction in ECOPD with daily azithromycin 250mg over 1 year in COPD patients who had been either hospitalized, treated with long-term oxygen or prescribed oral corticosteroids for an ECOPD in the preceding 12 months. ${ }^{105}$ This benefit was apparent 
even in patients already treated with ICS and bronchodilators. Uzen et al, randomized 94 patients with $\geq 3$ exacerbations in the previous year to azithromycin 500mg three times a week or placebo for 1 year. Azithromycin resulted in a significant reduction in the exacerbation rate versus placebo. ${ }^{106}$ Although a small study, all patients in this trial had persistent exacerbations despite optimal inhaled treatment and the study therefore established that macrolides can provide additive benefit for patients still experiencing exacerbations despite optimal "standard care". Finally, a meta-analysis studying eleven randomized controlled trials and one retrospective study compares the efficacy and safety of long-term prophylactic macrolide treatment to controls with standard care. The use of long-term macrolide treatment achieves a significant reduction of the total number of cases with one or more exacerbations and a lower rate of exacerbations per patient per year (risk ratio 0.60). Additionally, the group with the long-term macrolide treatment shows a significant improvement of St George Respiratory Questionnaire total score. The frequency of hospital admissions for ECOPD is not differ between the long-term macrolide treatment group and the controls. Subanalysis reveals that to achieve these improvements a minimum duration of 6 months macrolide treatment is required to obtain these effects. Clinicians have to be careful with the use of azithromycin as this drug is associated with increased bacterial resistance, prolonged QT interval and loss of hearing. ${ }^{107}$

In conclusion, although currently available interventions are considered evidence-based according standard criteria, effects on symptoms and risk reduction are small to moderate, resulting in a remaining high disease burden.

\section{Exacerbations of COPD: definition, aetiology and pathophysiology}

Exacerbations of COPD (ECOPD) are a pivotal healthcare problem worldwide, accounting for the majority of the COPD-related healthcare costs. ${ }^{1}$ In COPD periods of relative clinical stability are frequently interrupted by recurrent exacerbations defined as acute worsening of respiratory symptoms that requires changes in medication and/or hospitalization. ${ }^{1}$ These acute events or lung attacks frequently result in hospitalizations, accelerated disease progression, an important negative impact on patient's wellbeing, consumption of significant financial resources and a high mortality $(4-29 \%))^{1,13,108}$ 
Up to now a standard definition of exacerbation of COPD is not universally established. This absence of homogeneity in the definition of ECOPD hinders progress in clinical research. In the 80's Anthonisen formulated criteria to define ECOPD based on patients' symptoms. ${ }^{109}$ Anthonisen defined exacerbations as type 1 if they had all the major symptoms of increased dyspnea, sputum volume and sputum purulence, type 2 exacerbations with two of the above symptoms and type 3 exacerbations when one of the symptoms was combined with cough, wheeze or symptoms of an upper airway infection. ${ }^{109}$ The recent European Respiratory Society/ American Thoracic Society guidelines on the management of COPD exacerbations, define exacerbations as "episodes of increasing respiratory symptoms, particularly dyspnea, cough and sputum production and increased sputum purulence". ${ }^{110}$ In 2000, a consensus definition for COPD exacerbations was proposed: exacerbations were defined as "a sustained worsening of the patient's condition, from the stable state and beyond normal day-today variations, that is acute in onset and necessitates a change in regular medication in a patient with underlying COPD". ${ }^{111}$ In the absence of adequate biomarkers, limited studies have evaluated this day-to-day variability. Studies implementing daily measurements of oxygen saturation or forced oscillations reported important withinday and intra-day variability of these physiological variables in stable COPD patients. ${ }^{112,113}$ The clinical presentation of exacerbation of COPD can vary broadly and ranges from worsening of dyspnoea to devastating life threatening events. Categorization of ECOPD in mild, moderate and severe based on health care utilization of medication needs is proposed by GOLD ${ }^{8}$ :

Mild: increasing COPD symptoms for $>2$ consecutive days but not prescribed systemic corticosteroids or antibiotics

Moderate: increasing COPD symptoms leading to treatment with systemic corticosteroids or antibiotics

Severe: increasing COPD symptoms leading to hospitalization or emergency department stay $>24 \mathrm{hrs}$.

However, health care utilization in COPD varies from country to country and thus there may be considerable difficulty in standardisation of such definition. Furthermore, events not reported to healthcare professionals may not been captured and this would bias any definition based on access to health care. Otherwise, these unreported events may contribute to impaired health-related quality of life ${ }^{115,116}$ and increase the risk of hospital admission. ${ }^{117}$ Frequently used definitions of COPD exacerbations are summarized in Table $1.3 .^{114}$ All these definitions have a poor specificity and sensitivity. ${ }^{114}$ 
Table 1.3 Frequently used definitions and diagnostic criteria for COPD exacerbations.

\begin{tabular}{|c|c|}
\hline GOLD definition & $\begin{array}{l}\text { Acute exacerbations are episodes of acute worsening } \\
\text { of the respiratory symptoms of patients with COPD, } \\
\text { that result in additional therapy }\end{array}$ \\
\hline $\begin{array}{l}\text { Consensus conference } 2002 \text { (Rodriguez-Roisin) } \\
\text { definition }\end{array}$ & $\begin{array}{l}\text { In patients with underlying COPD, exacerbation is an } \\
\text { acute sustained symptoms worsening from the stable } \\
\text { state that is beyond normal day-to-day variation and } \\
\text { necessitates a change in regular medications }\end{array}$ \\
\hline $\begin{array}{l}\text { COPD-X } \\
\text { (Australian and New Zealand Guidelines) }\end{array}$ & $\begin{array}{l}\text { A COPD exacerbation is characterized by a change in } \\
\text { the patient's baseline dyspnea, cough, and/or sputum } \\
\text { that is beyond normal day-to-day variations, is acute } \\
\text { in onset, and may warrant a change in regular } \\
\text { medication or hospital admission }\end{array}$ \\
\hline NICE (UK Guidelines) & $\begin{array}{l}\text { An exacerbation is a sustained worsening of the } \\
\text { patient's symptoms from their usual stable state } \\
\text { which is beyond day-to-day variations and is acute in } \\
\text { onset. Commonly reported symptoms are worsening } \\
\text { breathlessness, cough, increased sputum production } \\
\text { and change in sputum colour. The change in these } \\
\text { symptoms often necessitates a change in medication }\end{array}$ \\
\hline Anthonisen criteria & $\begin{array}{l}\text { In patients with underlying COPD, an exacerbation is } \\
\text { an acute, sustained deterioration of at least two of } \\
\text { the following symptoms: Increased sputum volume; } \\
\text { increased sputum purulence; breathlessness }\end{array}$ \\
\hline Modified Anthonisen criteria & $\begin{array}{l}\text { In patients with underlying COPD, an exacerbation is } \\
\text { an acute sustained deterioration of at least two of the } \\
\text { following major symptoms or at least one major and } \\
\text { one minor symptom. Major symptoms: Increased } \\
\text { sputum volume; increased sputum purulence; } \\
\text { breathlessness. Minor symptoms: cough; wheeze; } \\
\text { nasal discharge; sore throat; pyrexia }\end{array}$ \\
\hline EXACT criteria & $\begin{array}{l}\text { The EXACT patient-reported diary is a patient- } \\
\text { reported outcome measure developed to identify } \\
\text { COPD exacerbations, by quantifying daily the ntensity } \\
\text { of the following symptoms: congestion, cough, } \\
\text { sputum production, sputum thickness, chest } \\
\text { discomfort, chest tightness, and breathlessness. An } \\
\text { exacerbation is defined by an increase of at least } 12 \\
\text { points for two consecutive days or an increase of at } \\
\text { least } 9 \text { points for at least three consecutive days }\end{array}$ \\
\hline
\end{tabular}

Abbreviations: COPD: Chronic obstructive pulmonary disease; EXACT: the exacerbations of chronic obstructive pulmonary disease tool; GOLD: Global initiative for chronic obstructive lung disease; NICE: National Institute for health and Care Excellence. Reference: Mathioudakis et al, acute exacerbations of chronic obstructive pulmonary disease: in search of diagnostic biomarkers and treatable traits. Thorax, $2020 .^{114}$

To achieve effective management strategies of COPD exacerbation a thorough and better understanding of the underlying pathophysiology and its clinical expression is urgently needed. Not reflected in current practice yet, growing consensus exits to 
consider evokes ECOPD as complex flare-ups of the disease with heterogeneous underlying mechanisms in response to different provoking stimuli. In an approach to dissect COPD exacerbations, system network analysis of 69 quantified variables including clinical, routine laboratory, microbiological, cytokines and imaging was conducted. ${ }^{118}$ The authors reported that during the acute phase of hospitalization for an exacerbation, there is a disruption of the organizational interaction that exists amongst all the variables grouped in modules compared to that observed after 3 months of recovery. Furthermore, the authors also observed that during the acute phase of the event, there was a measurable increase in the perception of dyspnoea. $^{118,119}$

COPD exacerbations have been associated with a number of etiological factors, including infection and pollution episodes (Table 1.4). Non-infectious conditions account for $15-20 \%$ of exacerbations including air pollution (Sulphur dioxide, Nitrogen dioxide, Ozone), meteorological effects and accompanied comorbidities of the patients like pulmonary embolism and heart failure. However, in up to $30 \%$ the cause of ECOPD remains unknown. ${ }^{120}$

Table 1.4 Causes of COPD exacerbations.

\begin{tabular}{lll}
\hline Viruses & Bacteria & Common pollutants \\
\hline Rhinovirus (common cold) & Haemophilus influenzae & Nitrogen dioxide \\
Influenza & Streptococcus pneumoniae & Particulates \\
Parainfluenza & Branhamella catarrhalis & Sulphur dioxide \\
Coronavirus & Staphylococcus aureus & Ozone \\
Adenovirus & Pseudomonas aeruginosa & \\
Respiratory syncytial virus & & \\
Chlamydia pneumoniae & & \\
\hline
\end{tabular}

COPD exacerbations are frequently triggered by upper respiratory infections, most common in winter months. In winter months, patients with COPD are also more prone to small but significant falls in lung function due to reduction in outdoor temperature. ${ }^{121}$ The precise role of bacterial infections is difficult to assess since bacterial colonization is associated with the same organisms as those isolated at exacerbations. ${ }^{120}$ Presentation with purulent sputum during an exacerbation of COPD is highly correlated with a bacterial infection of the lower respiratory tract. ${ }^{122}$ Bacteriae which play an important role in exacerbations are: Haemophilus influenzae (20-30\%), Streptococcus pneumoniae (10-15\%), Moraxella catarrhalis (10-15\%), Pseudomonas aeruginosa (5-10\% prevalent in advanced disease) and Enterobacteriaceae (in advanced disease). ${ }^{123}$ 
In one study in patients with moderate to severe COPD, bacteria were seen in $48.2 \%$ of patients in the stable state, raising to $69.6 \%$ at exacerbation with an associated rise in airway bacterial load. ${ }^{124}$ The presence of increased bacterial loads at exacerbation remains controversial. ${ }^{125,126}$ Bronchoscopic sampling revealed that bacteria were present in a clinical relevant concentration in the airways of $4 \%$ healthy persons, $29 \%$ stable COPD patients and in 54\% COPD patients with exacerbation. ${ }^{127}$ The presence of intracellular Haemophilus influenzae was found in $87 \%$ COPD patients who were intubated due to an exacerbation while Haemophilus influenzae could be detected in $33 \%$ of patients with stable COPD and in $0 \%$ of healthy controls. ${ }^{128}$ Others reported that the presence of potentially pathogenetic organisms in bronchoalveolar lavage from COPD patients was associated with a greater degree of inflammation. ${ }^{129}$ Evidence for the involvement of bacteria has come from studies of antibiotic therapy since exacerbations are often associated with increased purulence and volume and antibiotics have traditionally used as first line therapy. ${ }^{130}$ In 1987 , Anthonisen et al demonstrated that during exacerbation of COPD usage of antibiotics was associated with significant benefit and higher success rate, a lower treatment failure and faster recovery. ${ }^{109}$ Later on, the results of meta-analyses have shown that antibiotic therapy offered a small but significant benefit in treatment failure and mortality. ${ }^{131}$ Substantial progress has been made on investigating the role of bacterial infection at exacerbation by using molecular typing methods. These techniques allow detection of changes in bacterial strains, rather than species. ${ }^{132}$ Sethi and colleagues reported that in particular isolation of a new strain was associated with an increased risk of an exacerbation. However, this study does not prove causal relationship because not all exacerbations were associated with strain changes. ${ }^{133}$ The concept that bacteria are just innocent bystanders in the lower airways during exacerbations is further challenged by reports on strain-specific immune responses to colonizing bacterial species. ${ }^{134}$ Indeed, the outcome of acquisition of a bacterial strain depends on the interaction between the pathogenicity of bacteria and host factors. Genomic differences between strains of Haemophilus Influenzae will represent different outcomes and leading to different host response. ${ }^{135}$ Failure of the host to encounter the bacteria is also a key factor in the pathogenesis of an exacerbation. Insufficient innate immune system in COPD patients (host) will develop proliferation and colonization of bacteria in the airways. ${ }^{123}$ Compared to non-bacterial exacerbations bacterial exacerbations lead to higher systemic inflammation which is the highest with acquisition of new bacterial strain. ${ }^{136}$

Lung microbiota alterations are now also incriminated as potential causes of exacerbations. Modern molecular sequencing techniques have revealed that the healthy respiratory tract is colonized by microbiota consisting of complex bacterial 
communities. ${ }^{137}$ The microbiota of COPD are characterized by an outgrowth of the Proteobacteria phylum and an increase in the proportion of Streptococci and Staphylococci within the Firmicutes phylum. ${ }^{137-139}$ Recent studies reported an expansion of the Proteobacteria (Moraxella) within the microbiota at exacerbation. ${ }^{140,141}$ Further studies are needed to understand how shifts in microbiota may lead to exacerbation and to understand the immune mechanisms for these changes.

COPD exacerbations are frequently triggered by upper respiratory tract infections. Exacerbations triggered by respiratory viral infections are more severe and associated with longer recovery times than those triggered by other factors. ${ }^{142,143}$ Exacerbations triggered by respiratory viruses also have more chance of hospital admission than exacerbations where respiratory viruses were not detected. ${ }^{144}$ Molecular diagnostic techniques have now enabled detection of respiratory viruses at exacerbation, which have been isolated in around half of exacerbations ${ }^{142,143}$, but the precise proportion of exacerbations due to viruses is hardly to assess. ${ }^{123}$ The most common viruses isolated are human rhinoviruses, and other viruses including coronavirus, respiratory syncytial virus, influenza-, parainfluenza- and adenovirus. ${ }^{120,127}$ The precise mechanism how viral infections cause an exacerbation is still not fully understood.

In the last decade, there is growing interest in the eosinophilic COPD phenotype in order to allow more targeted treatment strategies. Until recently, COPD was considered as primarily a neutrophilic-mediated inflammatory disease. ${ }^{145}$ However, $20 \%-40 \%$ of patients with COPD have an eosinophilic airway inflammation, both during stable disease and during exacerbations. ${ }^{146-149}$ In stable COPD, a higher blood eosinophilic count is associated with an increased risk for future exacerbations ${ }^{150}$ as well with a better response to corticosteroid therapy in both stable COPD and during exacerbations. $^{151-153}$ COPD exacerbations characterized by enhanced airway eosinophilic inflammation are generally milder, reflected in lower mortality and shorter length of hospital stay. ${ }^{65,154}$ Otherwise, eosinophilia in inflammatory airway disease is reported to be associated with increased all cause-mortality. ${ }^{155,156}$ All these data probably highlight different genetic, biologic and pathologic processes.

The heterogeneity of exacerbations of COPD was clearly illustrated by the study of Bafadhel et al, published in 2011: 145 patients with COPD were observed for 1 year at stable and exacerbation visits and biomarkers were measured in sputum and serum. Four distinct biologic exacerbation clusters were identified: a bacterial, a viral, an eosinophilic-predominant as well as a pauci-inflammatory cluster: $28 \%$ of exacerbations were associated with sputum eosinophilia. ${ }^{146}$ The usefulness of blood eosinophils to 
direct corticosteroid therapy during exacerbations was proven by the same authors in a consecutive study. ${ }^{153}$ This randomized study evaluating 166 mostly moderate severe exacerbations demonstrated the safety of withholding systemic corticosteroids in those characterized by a blood eosinophilic count of less than $2 \%$ of total white blood cell count at baseline. The preferable use of additional eosinophilic measurements to guide systemic corticosteroids administration is also confirmed by the Corticosteroid Reduction in COPD (CORTICO-COP) trial. ${ }^{152}$ These data indicate that a biomarkerdirected strategy using the peripheral blood eosinophil count can be used to direct corticosteroid treatment during acute exacerbations of COPD and allows the identification of subgroups that have benefit and detriment from the use of this therapy. ${ }^{152}$

Besides understanding of the triggering mechanisms leading to an exacerbation, understanding of the underlying pathophysiological consequences is critical to implement effective targeted management strategies.

Exacerbations fundamentally reflect acute worsening of Expiratory Flow Limitation (EFL) by both increased airway inflammatory activity and acute worsening of airway obstruction resulting in abrupt increases of airways resistance. ${ }^{157}$ The time constant for lung emptying will be prolonged resulting in dynamic increases of End-Expiratory Lung Volume (EELV). Rapid shallow breathing, anxiety or transient hypoxemia will all contribute to increase in EELV (Figure 1.4). ${ }^{158}$ This acute Dynamic Hyperinflation (DH) has devastating negative effects on respiratory mechanics and cardiac dysfunction as depicted in Figure 1.5. ${ }^{158}$

Figure 1.4 Changes in lung volume during exercise $(A)$ in healthy subjects and in (B) in patients with chronic obstructive pulmonary diseases.

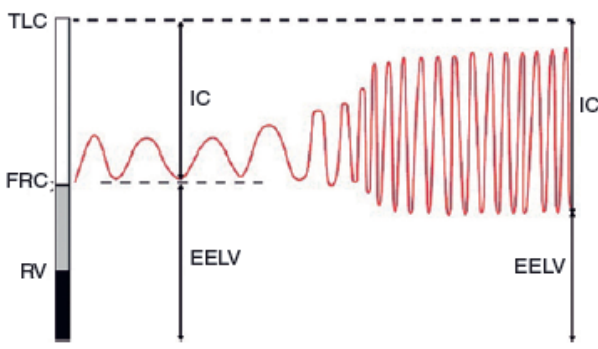

B

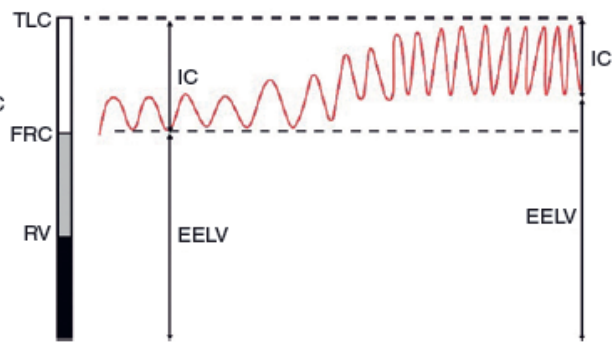

Abbreviations: EELV, end-expiratory lung volume; FRC: functional residual capacity; IC: inspiratory capacity; RV: residual volume; TLC: total long capacity. Reference: Garcia-Rio F., Lung hyperinflation in chronic obstructive pulmonary disease: clinical and therapeutic relevance. Barcelona Respiratory Network reviews, 2020. ${ }^{159}$ 
Figure 1.5 The negative consequences of dynamic hyperinflation during an acute exacerbation of COPD. Dynamic hyperinflation develops as a consequence of worsening expiratory flow limitation.

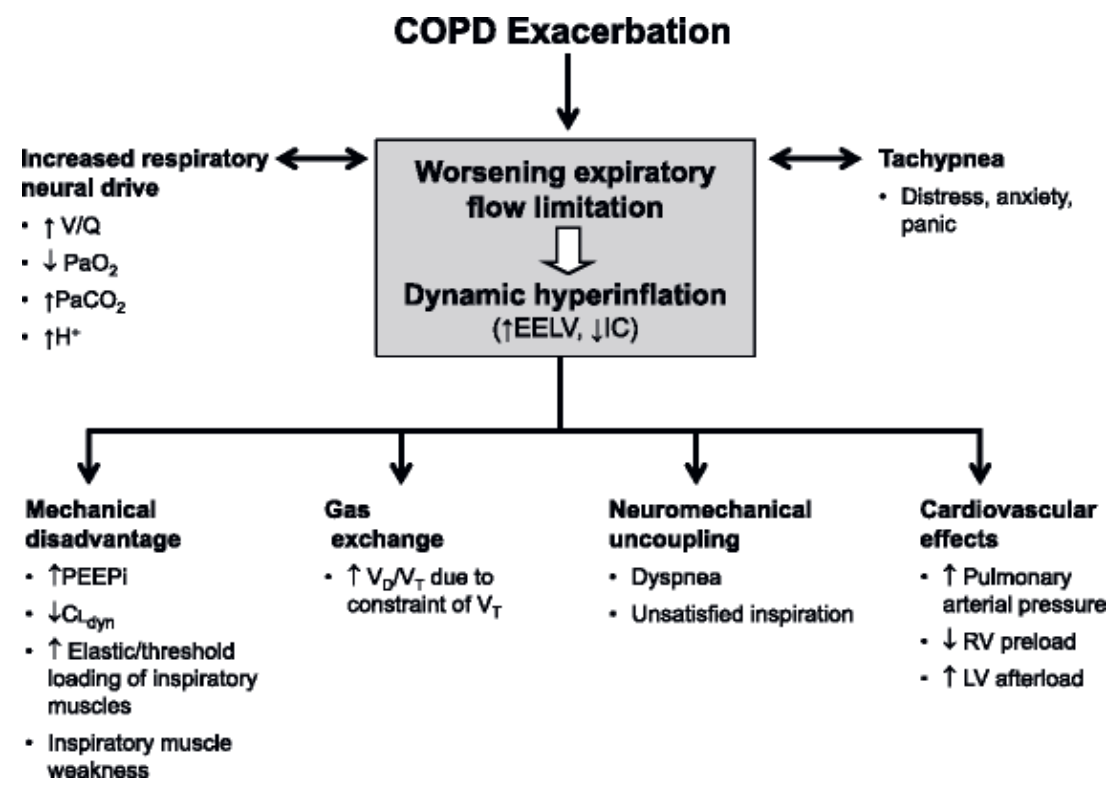

Abbreviations: EELV, end-expiratory lung volume; IC, inspiratory capacity; $\mathrm{PaO}_{2}$, arterial oxygen tension; $\mathrm{PaCO}_{2}$, arterial carbon dioxide tension; $\mathrm{PEEPi}$, intrinsic positive end-expiratory pressure; $\mathrm{CL}_{\text {dyn }}$, dynamic lung compliance; $\mathrm{H}^{+}$, hydrogen ion concentration; $\mathrm{LV}$, left ventricular $\mathrm{RV}$, right ventricular; $\mathrm{V}_{\mathrm{D}} / \mathrm{V}_{\mathrm{T}}$, physiological dead space; $\mathrm{V} / \mathrm{Q}$, ventilation/perfusion; $\mathrm{V}_{\mathrm{T}}$, tidal volume. Reference: O’Donnell; COPD exacerbations: 3/ pathophysiology. Thorax, $2003 .^{158}$

Dynamic Hyperinflation (DH) forces the respiratory system to operate on a flatter part of the compliance curve where progressive pressure increases will generate smaller incremental volume changes (Figure 1.6). ${ }^{159}$ Severe $\mathrm{DH}$ imposes a restrictive mechanical constraint on the respiratory system. Furthermore, where ventilatory muscles partially adapt to chronic hyperinflation to preserve the force generating capacity during resting breathing, acute $\mathrm{DH}$ will overwhelm these adaptations. ${ }^{160,161}$ This results in increased burden of the respiratory muscles by increased elastic loading on top of increased resistive loading. In addition, acute $\mathrm{DH}$ shortens the inspiratory muscles, resulting in functional muscle weakness. As a consequence of EFL, intrapulmonary pressures are positive at the end of expiration representing Intrinsic or auto-Positive End Expiratory Pressure (PEEPi): this PEEPi will act as an inspiratory threshold. During acute on chronic hyperinflation in COPD exacerbations the tachypnea will result in reduced dynamic compliance as a reflection of the time constant inequalities of alveolar units throughout the lungs. ${ }^{158}$ The net effect of this increased loading and functional weakness of the 
inspiratory muscles is that the effort required for tidal inspiration represents a relatively high fraction of the maximal possible effort that the patient can develop at that lung volume. On the other hand, neural drive to breathe is preserved or is increased. This central drive further increases in the presence of critical arterial oxygen desaturation, carbon dioxide retention and/or acidosis, by fever and by increased sympathetic nervous system activation. This imbalance between mechanical output and neural drive results in neuro-mechanical dissociation or uncoupling of the respiratory system and is perceived as dyspnea. ${ }^{158,162-165}$ Patients with severe or advanced disease with less physiologic reserve are prone to develop respiratory failure as a consequence of these mechanical disadvantages.

Figure 1.6 Schematic representation of static lung volumes and pressure-volume curves in normal subjects and in OCPD patient with hyperinflation.

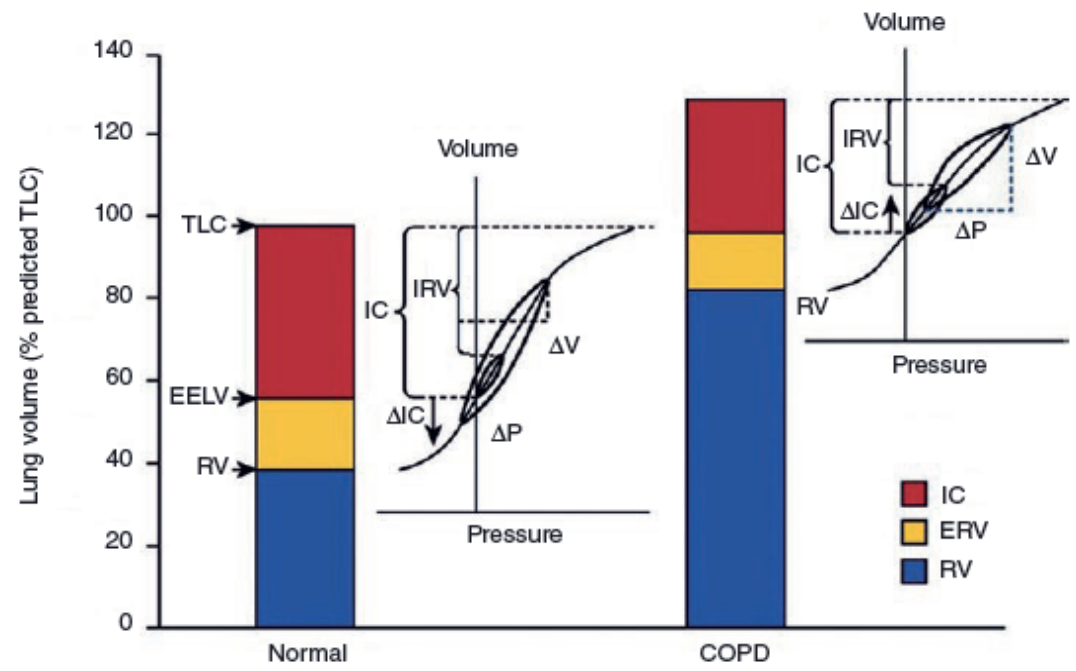

Abbreviations: COPD: chronic obstructive pulmonary diseases; EELV, end-expiratory lung volume; IC, inspiratory capacity; IRV: inspiratory reserve volume; ERV: expiratory reserve volume; PV: pressure-volume; RV: residual volume; TLC: total long capacity; Delta IC: change in IC from rest to exercise; Delta P: change in pleural pressure during tidal breath during exercise: Delta $\mathrm{V}$ : change in volume during tidal breath during exercise. Reference: Garcia-Rio F., Lung hyperinflation in chronic obstructive pulmonary disease: clinical and therapeutic relevance. Barcelona Respiratory Network reviews, $2020{ }^{159}$

Acute respiratory failure in the setting of COPD is characterized by worsening hypoxaemia and often presents with variable degrees of carbon dioxide retention and acidemiae. ${ }^{158}$ Worsening of the ratio of ventilation/perfusion (V/Q) relationships with increased V/Q mismatching accounts for nearly $50 \%$ of the observed hypoxaemia. Another part can be explained by a reduction in the mixed venous oxygen tension 
reflecting increased oxygen consumption due to increased work of breathing. $\mathrm{CO}_{2}$ retention in these patients is also a consequence of worsening $V / Q$ mismatching rather than alveolar ventilation. ${ }^{166}$ This $\mathrm{V} / \mathrm{Q}$ mismatching will also increase the physiological dead space resulting in reduction of alveolar ventilation.

Acute development of PEEPi due to $\mathrm{DH}$ will reduce right ventricular (RV) preload due to impaired venous return to the right atrium. ${ }^{167} \mathrm{DH}$ may also increases pulmonary vascular resistance due to breathing at lung volumes close to total lung capacity (TLC). To note is that pulmonary artery pressures are already higher at any cardiac output in patients with COPD. ${ }^{168-171}$ Left ventricular diastolic function may also be adversely affected by the increases in right ventricular end diastolic pressures as a consequence of ventricular interdependence. This may impair left ventricular filling (Figure 1.7). ${ }^{172,173}$

Figure 1.7 Schematic diagram showing the potential deleterious effects of lung hyperinflation on cardiopulmonary interactions in patients with COPD. These interactions may vary according to phase alignment between the respiratory and cardiac cycles. There are also important modulating effects of volumic status, sympathetic nervous system activation, ventilation-related vagal reflexes and comorbidities (e.g., pulmonary hypertension and chronic heart failure) which are not depicted.

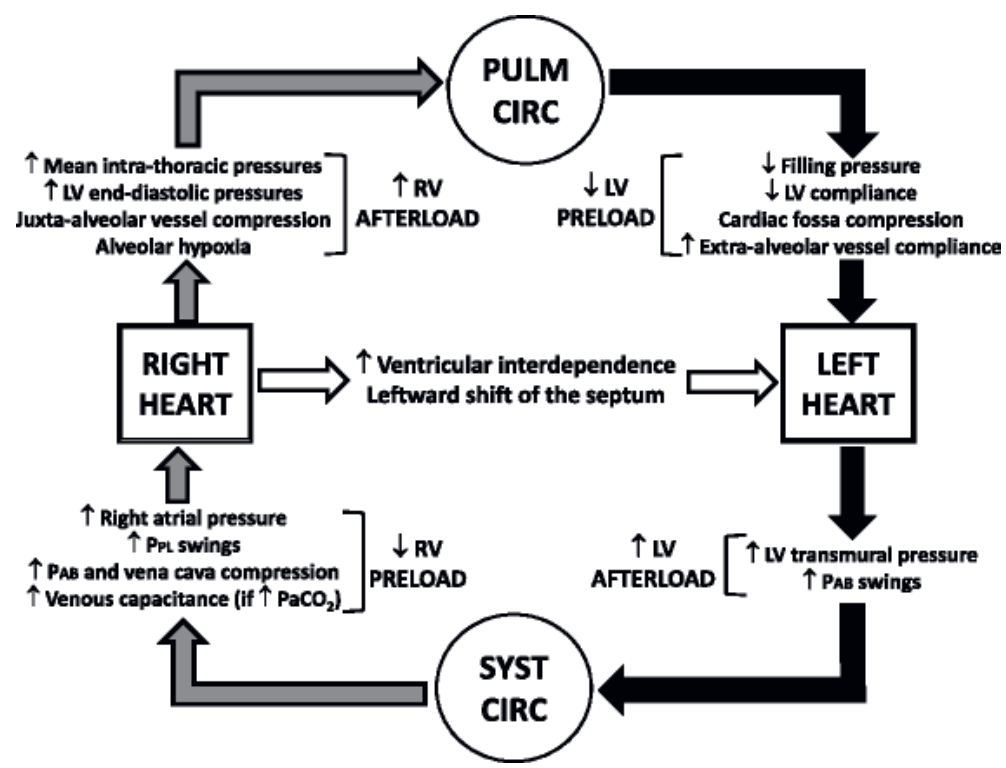

Abbreviations: Circ, circulation; LV, left ventricular; $\mathrm{Pab}$, abdominal pressure; $\mathrm{PaCO}_{2}$, partial pressure of arterial carbon dioxide; Ppl, pleural pressure; Pulm, pulmonary; Syst, systemic; RV, right ventricular. Reference: O'Donnell; Lung hyperinflation in COPD: applying physiology to clinical practice. COPD research and practice, 2015. 
In order to further improve personalized treatment for patients with COPD exacerbations, a two-axes classification was proposed by combination of a pathobiological axis with a clinical axis or severity score. ${ }^{174,175}$ Recently Van Geffen et al suggested to add hyperinflation as a third axis to better classify COPD exacerbations. Intriguingly, the same authors recently reported in a prospective observational cohort study that static hyperinflation is increased during severe ECOPD requiring hospitalization compared to stable state, but they could not found a metronome-paced dynamic hyperinflation increase. ${ }^{176}$ In fact, the authors conclude that they could not demonstrate a further increase in $\mathrm{DH}$ over and above the change in hyperinflation already induced by the exacerbation. The authors believe that these data support the suggestion to incorporate the occurrence of static hyperinflation during severe exacerbation along current parameters of inflammation and respiratory infections in a new definition of ECOPD.

Particularly during episodes of acute respiratory failure, changes in fluid homeostasis can contribute to edema. Both hypoxemia and hypercapnia can affect renal sodium excretion in severe COPD. Even in clinically stable hypercapnic patients impaired excretion of sodium and water is present and correlations have been found between the degree of hypercapnia and the impairment in sodium excretion. Renin and aldosterone concentrations are inversely correlated with total sodium loss, in both patients with severe COPD and those with acute respiratory failure. ${ }^{177-180}$ On the other hand, hypoxaemia causes a significant fall in urinary sodium output with no changes in water excretion, probably related to a decline in glomerular filtration rate. ${ }^{181}$ So, under conditions of hypoxaemia as well as hypercapnia kidneys of patients with COPD are in a sodium-retaining state. ${ }^{52,182}$ Severe hypoxaemia negatively affects renal blood flow and in the presence of hypercapnia renal perfusion progressively falls and this vasoconstrictor mechanism withstands vasodilatory stimuli. ${ }^{182-187}$

In conclusion, exacerbations seem very heterogeneous in biologic and mechanical characteristics. Understanding these underlying factors may help to offer more targeted and personalised treatment strategies for these disabling conditions.

\section{Hospitalizations and COPD exacerbations}

Patients admitted to the hospital with an acute exacerbation are at risk for future exacerbations, protracted recovery, impaired health status and death. Hospitalizations for ECOPD are frightening and create disruption to people's lives and that of the family 
members. ${ }^{188}$ Despite the personal and economical impact of these acute events, decision to admit a patient is still based on patient reported factors as the experienced sensation of dyspnea and non-specific clinical findings. Indeed, an objective marker of severity of ECOPD or an admission index for ECOPD is still lacking. Moreover, predictors of high likelihood of hospitalization are scarcely studied.

A systematic review conducted to identify potential risk factors for hospital admission and/or re-admission among patients experiencing ECOPD, could found no more than 17 articles that met predefined inclusion criteria but heterogeneity of study designs, risk factors and outcomes precluded a formal meta-analysis. ${ }^{189}$ Increased $\mathrm{PaCO}_{2}$ was shown to be an independent risk factor for hospital admission: this conclusion was based on the data of only a few studies. ${ }^{189-192}$ A study conducted to identify factors associated with hospital admission in patients reaching the emergency department with ECOPD identified impaired oxygenation, neutrophilia and prescription of antibiotics in the emergency department as factors associated with greater probability of admission. ${ }^{193}$ Another study looked at a prediction model for hospital admission due to ECOPD in subjects with COPD during primary care visits. Following predictive parameters for hospital admission were identified: age, sex, previous exacerbations, number of visits to the primary care center, comorbidities, smoking, disease severity and influenza immunization. $^{194}$

As reduction of early readmissions has become a policy target in many developed countries as a measure to judge both process and quality outcomes of care, concern is growing to identify risk factors for these early readmissions. ${ }^{195}$ Indeed, one of five patients admitted for an ECOPD are re-hospitalized within 30 days after discharge. ${ }^{196-198}$ A number of factors have been identified as increasing risk for early readmission after ECOPD (Table 1.5).

Table 1.5 Factors associated with increased risk for early readmission for exacerbation after index admission for exacerbation of COPD.

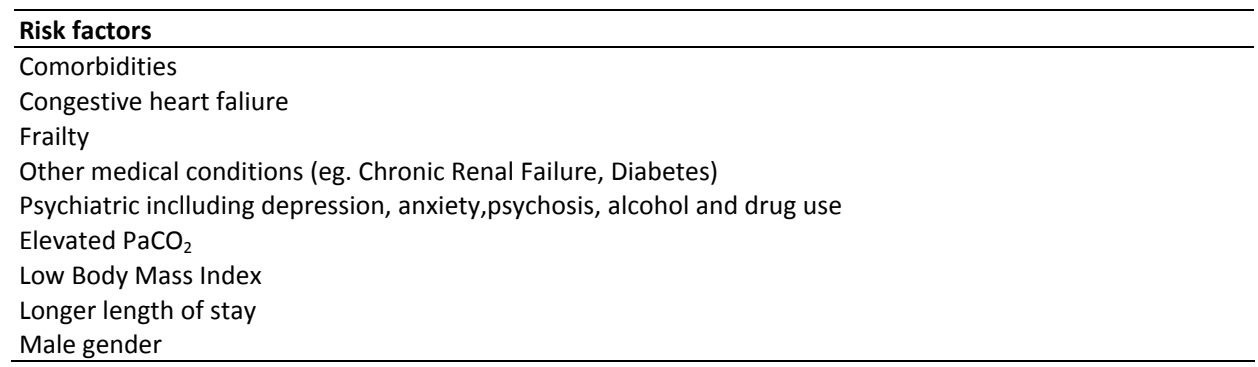


Particularly, the role of comorbidities is overlooked in clinical practice: congestive heart failure, anxiety, depression, obesity, osteoporosis, chronic kidney disease, diabetes mellitus, hypertension and obstructive sleep apnea are all related to higher rates of readmission. ${ }^{199,200}$ Congestive heart failure and osteoporosis are found to have the greatest risk of readmission. ${ }^{200}$ The number of comorbidities is related to a stepwise increase in the rate of readmissions: the rate of readmission increases by $47 \%$ by every single unit of increase in comorbidities. ${ }^{201}$ Another key factor for readmission is frailty: frail patients have significantly greater risk of hospitalization, disability and death. ${ }^{202,203}$ A more recent Korean study reported a readmission rate of $26.4 \%$ among 15101 studied patients and over 50\% of these readmissions occurred within 10 days of discharge. Remarkably, major respiratory causes of readmission were COPD, pneumonia and lung cancer. Risk factors for readmission within 30 days of discharge in that study were male sex, longer hospital stay, longer duration of systemic steroid use during hospital stay, high comorbid condition index and discharge to skilled nursing facilities. $^{198}$ Clear markers to monitor the treatment of ECOPD and to predict readmission are still lacking. The PEARL (Previous admissions Extended Medical Research Council dyspnea score, Age, Right-sided heart failure, Left-sided heart failure) is currently one of the most accurate scores to stratify patient's risk of readmission and death. ${ }^{204}$ Future predictive scores are needed to address the multifactorial and comorbid underlying pathology. ${ }^{205}$ ECOPD care pathways, the use of multifaceted care bundles, readmissions programs, a treatable traits approach and other initiatives are urgently needed to tackle the enormous ECOPD burden but the way to truly deliver value-based health care to these patients is still very long. ${ }^{188,205,206}$

The important role of hypercapnia in ECOPD admissions is now widely accepted. ${ }^{207-209}$ The application of Non-Invasive Ventilation (NIV) for acute hypercapnic respiratory failure is one of the major improvements and breakthroughs in threating hospitalizedECOPD accompanied with Acute Respiratory Failure (ARF). Treatment with (NIV is strongly recommended in patients with ECOPD accompanied with ARF leading to acute or acute on chronic respiratory acidosis $(\mathrm{pH} \leq 7.35) .{ }^{210}$ NIV has to be considered in patients with Hospitalized-ECOPD (H-ECOPD) developing respiratory failure in particularly when $\mathrm{pH} \leq 7.35, \mathrm{PaCO}_{2}>45 \mathrm{mmHg}$ and the respiratory rate $>20-24$ breaths/minute despite standard medical care. ${ }^{210-212}$ Using NIV the mechanistic advantage consists of unloading the respiratory muscles and reducing the work of breathing. ${ }^{211}$ Usage of NIV may help to avoid endotracheal intubation and prevent deterioration to a point when invasive ventilation is required. ${ }^{213}$ The severity of hypercapnia and acidosis at admission are associated with early treatment failure. ${ }^{214,215}$ On the other hand improvements in acidosis and breathing rate after four hours use of 
NIV were related to successful outcome. Confalonieri et al established a comprehensive chart of risk describing the probability of failure in patients admitted with ECOPD with ARF treated with NIV. ${ }^{216}$ The investigators based their risk stratification of NIV failure on assessment on four factors: Glasgow coma scale (GSC), Acute Physiology and Chronic Health Evaluation (APACHE) II, respiratory rate and $\mathrm{pH}$ at admission (before start NIV). These clinical symptoms were assessed in 1.033 patients of whom 797 patients were successfully treated with NIV. Patients with GSC $<11$ points, APACHE II $\geq 29$, respiratory rate $\geq 30$ breaths/min, and $\mathrm{pH}$ at admission $<7.25$ had a predicted risk of failure treatment of NIV of more than $70 \%$. Lower $\mathrm{pH}$ than 7.25 after two hours monitoring will worsening the outcome (Figure 1.8). ${ }^{216}$

Figure 1.8 Failure risk chart of non-invasive positive pressure ventilation at admission (the values in the table correspond to the percentage of patients who fail in each category: green: 0-24\%; yellow: 25-49\%; orange: $50-74 \%$; red: $75-100 \%$ ).

\begin{tabular}{|c|c|c|c|c|c|c|c|}
\hline & \multirow[b]{2}{*}{ RR } & \multicolumn{2}{|c|}{$\mathrm{pH}$ admission $<7.25$} & \multicolumn{2}{|c|}{$\mathrm{pH}$ admission $7.25-7.29$} & \multicolumn{2}{|c|}{$\mathrm{pH}$ admission $>7.30$} \\
\hline & & APACHE $\geq 29$ & APACHE $<29$ & APACHE $\geq 29$ & APACHE $<29$ & APACHE $\geq 29$ & APACHE $<29$ \\
\hline \multirow{3}{*}{$\begin{array}{c}\text { GCS } \\
15\end{array}$} & $<30$ & 29 & 11 & 18 & 6 & 17 & 6 \\
\hline & $30-34$ & 42 & 18 & 29 & 11 & 27 & 10 \\
\hline & $\geq 35$ & 52 & 24 & 37 & 15 & 35 & 14 \\
\hline \multirow{3}{*}{$\begin{array}{c}\text { GCS } \\
12-14\end{array}$} & $<30$ & 48 & 22 & 33 & 13 & 32 & 12 \\
\hline & $30-34$ & 63 & 34 & 48 & 22 & 46 & 21 \\
\hline & $\geq 35$ & 71 & 42 & 57 & 29 & 55 & 27 \\
\hline \multirow{3}{*}{$\begin{array}{l}\text { GCS } \\
\leq 11\end{array}$} & $<30$ & 64 & 35 & 49 & 23 & 47 & 21 \\
\hline & $30-34$ & 76 & 49 & 64 & 35 & 62 & 33 \\
\hline & $\geq 35$ & 82 & 59 & 72 & 44 & 70 & 42 \\
\hline
\end{tabular}

Abbreviations: RR: respiratory rate; APACHE: acute physiology and chronic health evaluation II score; GCS: Glasgow Coma Scale. Reference: Confalonieri et al, A chart of failure risk for non-invasive ventilation in patient with COPD exacerbations. European Respiratory Journal 2005. ${ }^{216}$

Ventilator asynchrony is another major factor for uncomforting and failure of NIV treatment. This uncomforting ventilatory asynchrony is frequently overlooked. Adequate attention to flow and pressure waveforms related to patients thorax excursions and patients feelings are mandatory after every change in ventilatory settings to prevent NIV failure and to improve patient's outcome. ${ }^{217}$ Furthermore, selection of an appropriate fitting interface is also of major importance because of the vast leaks with unfitting masks will cause an insensitive trigger, affecting patient tolerance and synchrony. ${ }^{218}$

Reported adverse effects of NIV treatment are claustrophobia, skin abrasion over the application site of the mask interface, gastric distension, and pneumonia (less common 
as by invasive mechanical ventilation). ${ }^{219}$ Treatment with NIV in patients admitted for ECOPD with ARF may result in effective weaning within 2-10 days. ${ }^{220}$

Inhaled helium /oxygen mixture (Heliox) has been proposed as a adjunctive therapy to NIV in severe ECOPD. In theory Helium has a lower density compared to air and physiologically reduces the work of breathing and the level of $\mathrm{PaCO}_{2}$. Nevertheless, clinical trials in hypercapnic ECOPD patients did not show significant effects on clinical outcomes. $^{221}$ Indeed, higher viscosity of Heliox may negatively affect resistance in laminar flow areas as in the peripheral airways.

Despite the extensive use of NIV to treat patients with H-ECOPD and ARF, knowledge on long-term outcome is scarce. Patients who are admitted for ECOPD accompanied with acute hypercapnic respiratory failure and require mechanical ventilation have a very poor outcome regarding mortality and quality of life after hospital discharge. ${ }^{222-226}$ Chung et al demonstrated that advanced age, low BMI and prior domiciliary oxygen use are associated with death within five years in COPD patients surviving the first episode of respiratory failure requiring NIV. ${ }^{227}$ Further Chu et al reported in these patient group a readmission rate of more than $60 \%$ and a mortality rate of more than $25 \%$ within the first year after discharge. ${ }^{223}$ However, more studies are needed on predictive characteristics of in-hospital as well as of long-term mortality in these patients with acute respiratory failure requiring NIV. Therefore, it might be attractive to continue the NIV at home after discharge of a ECOPD with life-threatening acute respiratory failure, targeted to reduce and delay readmissions, lower the mortality rate and to improve the quality of life. In particular, the application of chronic nocturnal NIV in patients admitted for ECOPD who remain hypercapnic after an episode of invasive or noninvasive ventilatory support during ARF is poorly documented. Some studies showed benefit in long-term outcomes using chronic NIV in patients who previously needed ventilatory support for treatment of ARF and persisted hypercapnia. ${ }^{228,229}$

In conclusion, despite the tremendous personal and economical burden, the high number of hospitalizations and readmission rates, ECOPD remain a conundrum for health care professionals and our current management is still largely experienced instead of knowledge based. Scanty information is derived from a limited number of observational studies. Just considering the high number of annual in-hospital deaths worldwide, ECOPD merits and urgently needs a systematic research approach to address the underlying disease mechanisms by better treatment regimens. 


\section{References}

1. Vogelmeier, C.F., et al., Global Strategy for the Diagnosis, Management, and Prevention of Chronic Obstructive Lung Disease 2017 Report. GOLD Executive Summary. Am J Respir Crit Care Med, 2017. 195(5): p. 557-582.

2. Fletcher, C. and R. Peto, The natural history of chronic airflow obstruction. Br Med J, 1977. 1(6077): p. 1645-8.

3. Lange, P., et al., Lung-Function Trajectories Leading to Chronic Obstructive Pulmonary Disease. N Engl J Med, 2015. 373(2): p. 111-22.

4. Lange, P., B. Celli, and A. Agusti, Lung-Function Trajectories and Chronic Obstructive Pulmonary Disease. N Engl J Med, 2015. 373(16): p. 1575.

5. Agusti, A., et al., Lung function in early adulthood and health in later life: a transgenerational cohort analysis. Lancet Respir Med, 2017. 5(12): p. 935-945.

6. Augustin, I.M.L., et al., The respiratory physiome: Clustering based on a comprehensive lung function assessment in patients with COPD. PLoS One, 2018. 13(9): p. e0201593.

7. Pauwels, R.A., et al., Global strategy for the diagnosis, management, and prevention of chronic obstructive pulmonary disease. NHLBI/WHO Global Initiative for Chronic Obstructive Lung Disease (GOLD) Workshop summary. Am J Respir Crit Care Med, 2001. 163(5): p. 1256-76.

8. GOLD, GOLD global strategy for the diagnosis. management and prevention of Chronic Obstructive Pulmonary disease Report https://goldcopd.org, 2020.

9. Rodriguez-Roisin, R., et al., Global Initiative for Chronic Obstructive Lung Disease (GOLD) 20th Anniversary: a brief history of time. Eur Respir J, 2017. 50(1).

10. Celli, B.R., et al., The body-mass index, airflow obstruction, dyspnea, and exercise capacity index in chronic obstructive pulmonary disease. N Engl J Med, 2004. 350(10): p. 1005-12.

11. Puhan, M.A., et al., Expansion of the prognostic assessment of patients with chronic obstructive pulmonary disease: the updated BODE index and the ADO index. Lancet, 2009. 374(9691): p. 704-11.

12. Hurst, J.R., et al., Susceptibility to exacerbation in chronic obstructive pulmonary disease. N Engl J Med, 2010. 363(12): p. 1128-38.

13. Han, M.K., et al., Frequency of exacerbations in patients with chronic obstructive pulmonary disease: an analysis of the SPIROMICS cohort. Lancet Respir Med, 2017. 5(8): p. 619-626.

14. Smid, D.E., et al., Redefining Cut-Points for High Symptom Burden of the Global Initiative for Chronic Obstructive Lung Disease Classification in 18,577 Patients With Chronic Obstructive Pulmonary Disease. J Am Med Dir Assoc, 2017. 18(12): p. 1097 e11-1097 e24.

15. Wilke, S., et al., The 2014 Updated GOLD Strategy: A Comparison of the Various Scenarios. Chronic Obstr Pulm Dis, 2014. 1(2): p. 212-220.

16. Tan, W.C., et al., Exacerbation-like respiratory symptoms in individuals without chronic obstructive pulmonary disease: results from a population-based study. Thorax, 2014. 69(8): p. 709-17.

17. Bowler, R.P., et al., Prediction of acute respiratory disease in current and former smokers with and without COPD. Chest, 2014. 146(4): p. 941-950.

18. Woodruff, P.G., D. Couper, and M.K. Han, Symptoms in Smokers with Preserved Pulmonary Function. N Engl J Med, 2016. 375(9): p. 896-7.

19. Regan, E.A., et al., Genetic epidemiology of COPD (COPDGene) study design. COPD, 2010. 7(1): p. 32-43.

20. Regan, E.A., et al., Clinical and Radiologic Disease in Smokers With Normal Spirometry. JAMA Intern Med, 2015. 175(9): p. 1539-49.

21. Tan, W.C., et al., Findings on Thoracic Computed Tomography Scans and Respiratory Outcomes in Persons with and without Chronic Obstructive Pulmonary Disease: A Population-Based Cohort Study. PLoS One, 2016. 11(11): p. e0166745.

22. Woodruff, P.G., et al., Clinical Significance of Symptoms in Smokers with Preserved Pulmonary Function. N Engl J Med, 2016. 374(19): p. 1811-21.

23. Adeloye, D., et al., Global and regional estimates of COPD prevalence: Systematic review and metaanalysis. J Glob Health, 2015. 5(2): p. 020415. 
24. Varmaghani, M., et al., Global prevalence of chronic obstructive pulmonary disease: systematic review and meta-analysis. East Mediterr Health J, 2019. 25(1): p. 47-57.

25. Gibson, G.J., et al., Respiratory health and disease in Europe: the new European Lung White Book. Eur Respir J, 2013. 42(3): p. 559-63.

26. Lopez-Campos, J.L., W. Tan, and J.B. Soriano, Global burden of COPD. Respirology, 2016. 21(1): p. 1423.

27. Buist, A.S., et al., International variation in the prevalence of COPD (the BOLD Study): a populationbased prevalence study. Lancet, 2007. 370(9589): p. 741-50.

28. Afonso, A.S., et al., COPD in the general population: prevalence, incidence and survival. Respir Med, 2011. 105(12): p. 1872-84.

29. Vanfleteren, L.E., et al., The prevalence of chronic obstructive pulmonary disease in Maastricht, the Netherlands. Respir Med, 2012. 106(6): p. 871-4.

30. van Durme, Y., et al., Prevalence, incidence, and lifetime risk for the development of COPD in the elderly: the Rotterdam study. Chest, 2009. 135(2): p. 368-377.

31. Volksgezondheidenzorg.info, https://www.volksgezondheidenzorg.info/onderwerp/copd, COPD.

32. Statistics, A.B.o., https://www.abs.gov.au/statistics/health.

33. Smid, D.E., et al., Burden of COPD in patients treated in different care settings in the Netherlands. Respir Med, 2016. 118: p. 76-83.

34. Spence, A., et al., Active carers: living with chronic obstructive pulmonary disease. Int J Palliat Nurs, 2008. 14(8): p. 368-72.

35. Gautun, H., A. Werner, and H. Luras, Care challenges for informal caregivers of chronically ill lung patients: results from a questionnaire survey. Scand J Public Health, 2012. 40(1): p. 18-24.

36. Hynes, G., A. Stokes, and M. McCarron, Informal care-giving in advanced chronic obstructive pulmonary disease: lay knowledge and experience. J Clin Nurs, 2012. 21(7-8): p. 1068-77.

37. Kuhl, K., W. Schurmann, and W. Rief, Mental disorders and quality of life in COPD patients and their spouses. Int J Chron Obstruct Pulmon Dis, 2008. 3(4): p. 727-36.

38. Gabriel, R., et al., Day-to-day living with severe chronic obstructive pulmonary disease: towards a family-based approach to the illness impacts. Psychol Health, 2014. 29(8): p. 967-83.

39. Bergs, D., "The Hidden Client"--women caring for husbands with COPD: their experience of quality of life. J Clin Nurs, 2002. 11(5): p. 613-21.

40. 2017, F.f.t.G.B.o.D.S., http://www.healthdata.org/policy-report/findings-global-burden-disease-study2017.

41. Centers for Disease, C.a.p., COPD, https://www.cdc.gov/copd.

42. American Lung Association, t.i.C., https://www.lung.org/research/trends-in-lung-disease, 2013.

43. Guarascio, A.J., et al., The clinical and economic burden of chronic obstructive pulmonary disease in the USA. Clinicoecon Outcomes Res, 2013. 5: p. 235-45.

44. Welfare, A.I.o.H.a., Chronic obstructive pulmonary disease (COPD), associated comorbidities and risk factors. https://www.aihw.gov.au/reports/chronic-respiratory-conditions/copd-associated-comorbiditiesrisk-factors/related-material.

45. book, E.I.w., https: //www.erswhi teboo k.org/chapt ers/chron ic-obstr uctiv e-pulmo nary-disea. 2013.

46. Anees Ur, R., et al., The economic burden of chronic obstructive pulmonary disease (COPD) in the USA, Europe, and Asia: results from a systematic review of the literature. Expert Rev Pharmacoecon Outcomes Res, 2019: p. 1-12.

47. Rutten van-Molken, M.P. and T.L. Feenstra, The burden of asthma and chronic obstructive pulmonary disease: data from The Netherlands. Pharmacoeconomics, 2001. 19 Suppl 2: p. 1-6.

48. Srivastava, K., et al., Systematic review of humanistic and economic burden of symptomatic chronic obstructive pulmonary disease. Pharmacoeconomics, 2015. 33(5): p. 467-88.

49. Polatli, M., et al., Chronic obstructive pulmonary disease and associated healthcare resource consumption in the Middle East and North Africa: the BREATHE study. Respir Med, 2012. 106 Suppl 2: p. S75-85.

50. Wouters, E.F., The burden of COPD in The Netherlands: results from the Confronting COPD survey. Respir Med, 2003. 97 Suppl C: p. S51-9. 
51. Kirsch, F., et al., Direct and indirect costs of COPD progression and its comorbidities in a structured disease management program: results from the LQ-DMP study. Respir Res, 2019. 20(1): p. 215.

52. Wouters, E.F., Management of severe COPD. Lancet, 2004. 364(9437): p. 883-95.

53. Foo, J., et al., Continuing to Confront COPD International Patient Survey: Economic Impact of COPD in 12 Countries. PLoS One, 2016. 11(4): p. e0152618.

54. Anita W.M. Suijkerbuijk, G.A.A.d.W., Alet H. Wijga, Monique J.W.M. Heijmans, Martine Hoogendoorn, Maureen P.M.H. Rutten-van Mölken, Erica E.M. Maurits, Rudolf T. Hoogenveen en Talitha L. Feenstra, Maatschappelijke kosten van astma, COPD en respiratoire allergie. Ned Tijdschr Geneeskd, 2013. 2013;157:A6562

55. Gijsen R., P.M., Slobbe L., Mulder M., in het Panhuis M., Hoeymans N.,, Een nieuwe selectie van ziekten voor de Volksgezondheid Toekomst Verkenningen. RIVM rapport, 2013.

56. WHO Chronic Obstructive Pulmonary Disease (COPD). 2016. www.who.int/respiratory/copd/en/.

57. Wouters, E., F,M, The Lung-Gut-Cross Talk in Chronic Airways Diseases. Barcelona respiratory Network reviews, 2019. 5(1): p. 19-34.

58. Mathers, C.D. and D. Loncar, Projections of global mortality and burden of disease from 2002 to 2030. PLoS Med, 2006. 3(11): p. e442.

59. (WHO), W.H.O., Global Health Observatory (GHO) data; Top 10 causes of death. https://www.who.int/gho/mortality_burden_disease/causes_death/top_10/en/.

60. Mortality, G.B.D. and C. Causes of Death, Global, regional, and national life expectancy, all-cause mortality, and cause-specific mortality for 249 causes of death, 1980-2015: a systematic analysis for the Global Burden of Disease Study 2015. Lancet, 2016. 388(10053): p. 1459-1544.

61. Collaborators, G.B.D.C.R.D., Global, regional, and national deaths, prevalence, disability-adjusted life years, and years lived with disability for chronic obstructive pulmonary disease and asthma, 1990-2015: a systematic analysis for the Global Burden of Disease Study 2015. Lancet Respir Med, 2017. 5(9): p. 691-706.

62. Kochanek, K.D., et al., Mortality in the United States, 2016. NCHS Data Brief, 2017(293): p. 1-8.

63. Prevention, C.f.D.C.a. https://www.cdc.gov/nchs/products/databriefs/db 293.htm.

64. Groenewegen, K.H., A.M. Schols, and E.F. Wouters, Mortality and mortality-related factors after hospitalization for acute exacerbation of COPD. Chest, 2003. 124(2): p. 459-67.

65. Steer, J., J. Gibson, and S.C. Bourke, The DECAF Score: predicting hospital mortality in exacerbations of chronic obstructive pulmonary disease. Thorax, 2012. 67(11): p. 970-6.

66. Roberts, C.M., et al., Co-morbidities and 90-day outcomes in hospitalized COPD exacerbations. COPD, 2011. 8(5): p. 354-61.

67. Tabak, Y.P., et al., Development and validation of a mortality risk-adjustment model for patients hospitalized for exacerbations of chronic obstructive pulmonary disease. Med Care, 2013. 51(7): p. 597605.

68. Almagro, P., et al., Short- and medium-term prognosis in patients hospitalized for COPD exacerbation: the CODEX index. Chest, 2014. 145(5): p. 972-980.

69. Kart, L., et al., COPD: an underdiagnosed disease at hospital environment. Wien Klin Wochenschr, 2014. 126(3-4): p. 73-8.

70. Janssen, D.J., et al., Symptoms, comorbidities, and health care in advanced chronic obstructive pulmonary disease or chronic heart failure. J Palliat Med, 2011. 14(6): p. 735-43.

71. Gore, J.M., C.J. Brophy, and M.A. Greenstone, How well do we care for patients with end stage chronic obstructive pulmonary disease (COPD)? A comparison of palliative care and quality of life in COPD and lung cancer. Thorax, 2000. 55(12): p. 1000-6.

72. Mahler, D.A., et al., Impact of dyspnea and physiologic function on general health status in patients with chronic obstructive pulmonary disease. Chest, 1992. 102(2): p. 395-401.

73. Melani, A.S., Long-acting muscarinic antagonists. Expert Rev Clin Pharmacol, 2015. 8(4): p. 479-501.

74. Barnes, Bronchodilators: basic pharmacology. 1995: London: Chapman and Hal.

75. Oba, Y., et al., Dual combination therapy versus long-acting bronchodilators alone for chronic obstructive pulmonary disease (COPD): a systematic review and network meta-analysis. Cochrane Database Syst Rev, 2018. 12: p. CD012620. 
76. Kew, K.M., C. Mavergames, and J.A. Walters, Long-acting beta2-agonists for chronic obstructive pulmonary disease. Cochrane Database Syst Rev, 2013(10): p. CD010177.

77. Barnes, P.J., S.D. Shapiro, and R.A. Pauwels, Chronic obstructive pulmonary disease: molecular and cellular mechanisms. Eur Respir J, 2003. 22(4): p. 672-88.

78. Barnes, P.J., How corticosteroids control inflammation: Quintiles Prize Lecture 2005. Br J Pharmacol, 2006. 148(3): p. 245-54.

79. Seto, E. and M. Yoshida, Erasers of histone acetylation: the histone deacetylase enzymes. Cold Spring Harb Perspect Biol, 2014. 6(4): p. a018713.

80. Ito, K., et al., Decreased histone deacetylase activity in chronic obstructive pulmonary disease. N Engl J Med, 2005. 352(19): p. 1967-76.

81. Highland, K.B., C. Strange, and J.E. Heffner, Long-term effects of inhaled corticosteroids on FEV1 in patients with chronic obstructive pulmonary disease. A meta-analysis. Ann Intern Med, 2003. 138(12): p. $969-73$.

82. Soriano, J.B., et al., A pooled analysis of FEV1 decline in COPD patients randomized to inhaled corticosteroids or placebo. Chest, 2007. 131(3): p. 682-689.

83. Yang, I.A., et al., Inhaled corticosteroids for stable chronic obstructive pulmonary disease. Cochrane Database Syst Rev, 2012(7): p. CD002991.

84. Barnes, P.J., Scientific rationale for inhaled combination therapy with long-acting beta2-agonists and corticosteroids. Eur Respir J, 2002. 19(1): p. 182-91.

85. Tashkin, D.P. and N.J. Gross, Inhaled glycopyrrolate for the treatment of chronic obstructive pulmonary disease. Int J Chron Obstruct Pulmon Dis, 2018. 13: p. 1873-1888.

86. Wedzicha, J.A., et al., The prevention of chronic obstructive pulmonary disease exacerbations by salmeterol/fluticasone propionate or tiotropium bromide. Am J Respir Crit Care Med, 2008. 177(1): p. 19-26.

87. Singh, D., et al., Single inhaler triple therapy versus inhaled corticosteroid plus long-acting beta2agonist therapy for chronic obstructive pulmonary disease (TRILOGY): a double-blind, parallel group, randomised controlled trial. Lancet, 2016. 388(10048): p. 963-73.

88. Lipson, D.A., et al., Once-Daily Single-Inhaler Triple versus Dual Therapy in Patients with COPD. N Engl J Med, 2018. 378(18): p. 1671-1680.

89. Ferguson, G.T., et al., Triple therapy with budesonide/glycopyrrolate/formoterol fumarate with cosuspension delivery technology versus dual therapies in chronic obstructive pulmonary disease (KRONOS): a double-blind, parallel-group, multicentre, phase 3 randomised controlled trial. Lancet Respir Med, 2018. 6(10): p. 747-758.

90. Bafadhel, M., et al., Predictors of exacerbation risk and response to budesonide in patients with chronic obstructive pulmonary disease: a post-hoc analysis of three randomised trials. Lancet Respir Med, 2018. 6(2): p. 117-126.

91. Siddiqui, S.H., et al., Blood Eosinophils: A Biomarker of Response to Extrafine Beclomethasone/Formoterol in Chronic Obstructive Pulmonary Disease. Am J Respir Crit Care Med, 2015. 192(4): p. 523-5.

92. Papi, A., et al., Extrafine inhaled triple therapy versus dual bronchodilator therapy in chronic obstructive pulmonary disease (TRIBUTE): a double-blind, parallel group, randomised controlled trial. Lancet, 2018. 391(10125): p. 1076-1084.

93. Pascoe, S., et al., Blood eosinophil counts, exacerbations, and response to the addition of inhaled fluticasone furoate to vilanterol in patients with chronic obstructive pulmonary disease: a secondary analysis of data from two parallel randomised controlled trials. Lancet Respir Med, 2015. 3(6): p. 43542.

94. Vestbo, J., et al., Single inhaler extrafine triple therapy versus long-acting muscarinic antagonist therapy for chronic obstructive pulmonary disease (TRINITY): a double-blind, parallel group, randomised controlled trial. Lancet, 2017. 389(10082): p. 1919-1929.

95. Cheng, S.L. and C.H. Lin, Effectiveness using higher inhaled corticosteroid dosage in patients with COPD by different blood eosinophilic counts. Int J Chron Obstruct Pulmon Dis, 2016. 11: p. 2341-2348.

96. Dransfield, M.T., et al., Fluticasone furoate and vilanterol for COPD - authors' reply. Lancet Respir Med, 2013. 1(5): p. e21-2. 
97. Janson, C., et al., Scientific rationale for the possible inhaled corticosteroid intraclass difference in the risk of pneumonia in COPD. Int J Chron Obstruct Pulmon Dis, 2017. 12: p. 3055-3064.

98. Agusti, A., et al., Inhaled corticosteroids in COPD: friend or foe? Eur Respir J, 2018. 52(6).

99. Rabe, K.F., Update on roflumilast, a phosphodiesterase 4 inhibitor for the treatment of chronic obstructive pulmonary disease. Br J Pharmacol, 2011. 163(1): p. 53-67.

100. Rabe, K.F., et al., Anti-inflammatory effects of roflumilast in chronic obstructive pulmonary disease (ROBERT): a 16-week, randomised, placebo-controlled trial. Lancet Respir Med, 2018. 6(11): p. 827-836.

101. Martinez, F.J., et al., Effect of roflumilast on exacerbations in patients with severe chronic obstructive pulmonary disease uncontrolled by combination therapy (REACT): a multicentre randomised controlled trial. Lancet, 2015. 385(9971): p. 857-66.

102. Martinez, L., et al., Infectiousness of HIV-Seropositive Patients with Tuberculosis in a High-Burden African Setting. Am J Respir Crit Care Med, 2016. 194(9): p. 1152-1163.

103. Parnham, M.J., et al., Azithromycin: mechanisms of action and their relevance for clinical applications. Pharmacol Ther, 2014. 143(2): p. 225-45.

104. Dicker, A.J., et al., Neutrophil extracellular traps are associated with disease severity and microbiota diversity in patients with chronic obstructive pulmonary disease. J Allergy Clin Immunol, 2018. 141(1): p. 117-127.

105. Albert, R.K., et al., Azithromycin for prevention of exacerbations of COPD. N Engl J Med, 2011. 365(8): p. 689-98.

106. Uzun, S., et al., Azithromycin maintenance treatment in patients with frequent exacerbations of chronic obstructive pulmonary disease (COLUMBUS): a randomised, double-blind, placebo-controlled trial. Lancet Respir Med, 2014. 2(5): p. 361-8.

107. Cui, Y., et al., Long-term macrolide treatment for the prevention of acute exacerbations in COPD: a systematic review and meta-analysis. Int J Chron Obstruct Pulmon Dis, 2018. 13: p. 3813-3829.

108. Halpin, D.M., et al., Exacerbation frequency and course of COPD. Int J Chron Obstruct Pulmon Dis, 2012. 7: p. 653-61.

109. Anthonisen, N.R., et al., Antibiotic therapy in exacerbations of chronic obstructive pulmonary disease. Ann Intern Med, 1987. 106(2): p. 196-204.

110. Wedzicha, J.A.E.C.-C., et al., Management of COPD exacerbations: a European Respiratory Society/American Thoracic Society guideline. Eur Respir J, 2017. 49(3).

111. Rodriguez-Roisin, R., Toward a consensus definition for COPD exacerbations. Chest, 2000. 117(5 Suppl 2): p. 398S-401S.

112. Buekers, J., et al., Wearable Finger Pulse Oximetry for Continuous Oxygen Saturation Measurements During Daily Home Routines of Patients With Chronic Obstructive Pulmonary Disease (COPD) Over One Week: Observational Study. JMIR Mhealth Uhealth, 2019. 7(6): p. e12866.

113. Zimmermann, S.C., et al., Day-to-day variability of forced oscillatory mechanics for early detection of acute exacerbations in COPD. Eur Respir J, 2020. 56(3).

114. Mathioudakis, A.G., et al., Acute exacerbations of chronic obstructive pulmonary disease: in search of diagnostic biomarkers and treatable traits. Thorax, 2020. 75(6): p. 520-527.

115. Jones, P.W., et al., Characterisation and impact of reported and unreported exacerbations: results from ATTAIN. Eur Respir J, 2014. 44(5): p. 1156-65.

116. Xu, W., et al., Negative impacts of unreported COPD exacerbations on health-related quality of life at 1 year. Eur Respir J, 2010. 35(5): p. 1022-30.

117. Wilkinson, T.M., et al., Early therapy improves outcomes of exacerbations of chronic obstructive pulmonary disease. Am J Respir Crit Care Med, 2004. 169(12): p. 1298-303.

118. Noell, G., et al., Multi-level differential network analysis of COPD exacerbations. Eur Respir J, 2017. 50(3).

119. Celli, B.R., Dissecting COPD exacerbations: time to rethink our definition. Eur Respir J, 2017. 50(3).

120. Sapey, E. and R.A. Stockley, COPD exacerbations . 2: aetiology. Thorax, 2006. 61(3): p. 250-8.

121. Donaldson, G.C., et al., Effect of temperature on lung function and symptoms in chronic obstructive pulmonary disease. Eur Respir J, 1999. 13(4): p. 844-9.

122. Soler, N., et al., Bronchoscopic validation of the significance of sputum purulence in severe exacerbations of chronic obstructive pulmonary disease. Thorax, 2007. 62(1): p. 29-35. 
123. Sethi, S. and T.F. Murphy, Infection in the pathogenesis and course of chronic obstructive pulmonary disease. N Engl J Med, 2008. 359(22): p. 2355-65.

124. Wilkinson, T.M., et al., Effect of interactions between lower airway bacterial and rhinoviral infection in exacerbations of COPD. Chest, 2006. 129(2): p. 317-24.

125. Monso, E., et al., Risk factors for lower airway bacterial colonization in chronic bronchitis. Eur Respir J, 1999. 13(2): p. 338-42.

126. Wilson, R., Bacterial infection and chronic obstructive pulmonary disease. Eur Respir J, 1999. 13(2): p. 233-5.

127. Rosell, A., et al., Microbiologic determinants of exacerbation in chronic obstructive pulmonary disease. Arch Intern Med, 2005. 165(8): p. 891-7.

128. Bandi, V., et al., Nontypeable Haemophilus influenzae in the lower respiratory tract of patients with chronic bronchitis. Am J Respir Crit Care Med, 2001. 164(11): p. 2114-9.

129. Soler, N., et al., Airway inflammation and bronchial microbial patterns in patients with stable chronic obstructive pulmonary disease. Eur Respir J, 1999. 14(5): p. 1015-22.

130. Stockley, R.A., et al., Relationship of sputum color to nature and outpatient management of acute exacerbations of COPD. Chest, 2000. 117(6): p. 1638-45.

131. Ram, F.S., et al., Antibiotics for exacerbations of chronic obstructive pulmonary disease. Cochrane Database Syst Rev, 2006(2): p. CD004403.

132. Wedzicha, J.A. and T.A. Seemungal, COPD exacerbations: defining their cause and prevention. Lancet, 2007. 370(9589): p. 786-96.

133. Sethi, S., et al., New strains of bacteria and exacerbations of chronic obstructive pulmonary disease. $\mathrm{N}$ Engl J Med, 2002. 347(7): p. 465-71.

134. Yi, K., S. Sethi, and T.F. Murphy, Human immune response to nontypeable Haemophilus influenzae in chronic bronchitis. J Infect Dis, 1997. 176(5): p. 1247-52.

135. Chin, C.L., et al., Haemophilus influenzae from patients with chronic obstructive pulmonary disease exacerbation induce more inflammation than colonizers. Am J Respir Crit Care Med, 2005. 172(1): p. 85-91.

136. Sethi, S., et al., Inflammatory profile of new bacterial strain exacerbations of chronic obstructive pulmonary disease. Am J Respir Crit Care Med, 2008. 177(5): p. 491-7.

137. Hilty, M., et al., Disordered microbial communities in asthmatic airways. PLoS One, 2010. 5(1): p. e8578.

138. Sze, M.A., et al., The lung tissue microbiome in chronic obstructive pulmonary disease. Am J Respir Crit Care Med, 2012. 185(10): p. 1073-80.

139. Pragman, A.A., et al., The lung tissue microbiota of mild and moderate chronic obstructive pulmonary disease. Microbiome, 2018. 6(1): p. 7.

140. Wang, Z., et al., Sputum microbiome temporal variability and dysbiosis in chronic obstructive pulmonary disease exacerbations: an analysis of the COPDMAP study. Thorax, 2018. 73(4): p. 331-338.

141. Mayhew, D., et al., Longitudinal profiling of the lung microbiome in the AERIS study demonstrates repeatability of bacterial and eosinophilic COPD exacerbations. Thorax, 2018. 73(5): p. 422-430.

142. Seemungal, T., et al., Respiratory viruses, symptoms, and inflammatory markers in acute exacerbations and stable chronic obstructive pulmonary disease. Am J Respir Crit Care Med, 2001. 164(9): p. 1618-23.

143. Rohde, G., et al., Respiratory viruses in exacerbations of chronic obstructive pulmonary disease requiring hospitalisation: a case-control study. Thorax, 2003. 58(1): p. 37-42.

144. Greenberg, S.B., et al., Respiratory viral infections in adults with and without chronic obstructive pulmonary disease. Am J Respir Crit Care Med, 2000. 162(1): p. 167-73.

145. Fujimoto, K., et al., Airway inflammation during stable and acutely exacerbated chronic obstructive pulmonary disease. Eur Respir J, 2005. 25(4): p. 640-6.

146. Bafadhel, M., et al., Acute exacerbations of chronic obstructive pulmonary disease: identification of biologic clusters and their biomarkers. Am J Respir Crit Care Med, 2011. 184(6): p. 662-71.

147. Brightling, C.E., et al., Sputum eosinophilia and short-term response to prednisolone in chronic obstructive pulmonary disease: a randomised controlled trial. Lancet, 2000. 356(9240): p. 1480-5.

148. Singh, D., et al., Eosinophilic inflammation in COPD: prevalence and clinical characteristics. Eur Respir J, 2014. 44(6): p. 1697-700. 
149. Singh, S.J., et al., An official systematic review of the European Respiratory Society/American Thoracic Society: measurement properties of field walking tests in chronic respiratory disease. Eur Respir J, 2014. 44(6): p. 1447-78.

150. Vedel-Krogh, S., et al., Blood Eosinophils and Exacerbations in Chronic Obstructive Pulmonary Disease. The Copenhagen General Population Study. Am J Respir Crit Care Med, 2016. 193(9): p. 965-74.

151. Siva, R., et al., Eosinophilic airway inflammation and exacerbations of COPD: a randomised controlled trial. Eur Respir J, 2007. 29(5): p. 906-13.

152. Sivapalan, P., et al., Eosinophil-guided corticosteroid therapy in patients admitted to hospital with COPD exacerbation (CORTICO-COP): a multicentre, randomised, controlled, open-label, non-inferiority trial. Lancet Respir Med, 2019. 7(8): p. 699-709.

153. Bafadhel, M., et al., Blood eosinophils to direct corticosteroid treatment of exacerbations of chronic obstructive pulmonary disease: a randomized placebo-controlled trial. Am J Respir Crit Care Med, 2012. 186(1): p. 48-55.

154. Bafadhel, M., et al., Blood Eosinophils and Outcomes in Severe Hospitalized Exacerbations of COPD. Chest, 2016. 150(2): p. 320-8.

155. Hospers, J.J., et al., Asthma attacks with eosinophilia predict mortality from chronic obstructive pulmonary disease in a general population sample. Am J Respir Crit Care Med, 1999. 160(6): p. 1869-74.

156. Hospers, J.J., et al., Eosinophilia is associated with increased all-cause mortality after a follow-up of 30 years in a general population sample. Epidemiology, 2000. 11(3): p. 261-8.

157. Bhowmik, A., et al., Relation of sputum inflammatory markers to symptoms and lung function changes in COPD exacerbations. Thorax, 2000. 55(2): p. 114-20.

158. O'Donnell, D.E. and C.M. Parker, COPD exacerbations . 3: Pathophysiology. Thorax, 2006. 61(4): p. 35461.

159. Garcia-Rio, F., Lung Hyperinflation in Chronic Obstructive Pulmonary Disease: Clinical and Therapeutic Relevance. the Barcelona Respiratory Network Reviews, 2020. 6: p. 67-86.

160. Orozco-Levi, M., et al., Subcellular adaptation of the human diaphragm in chronic obstructive pulmonary disease. Eur Respir J, 1999. 13(2): p. 371-8.

161. Similowski, T., et al., Contractile properties of the human diaphragm during chronic hyperinflation. $\mathrm{N}$ Engl J Med, 1991. 325(13): p. 917-23.

162. Chen, Z., F.L. Eldridge, and P.G. Wagner, Respiratory-associated thalamic activity is related to level of respiratory drive. Respir Physiol, 1992. 90(1): p. 99-113.

163. De Troyer, A., et al., Neural drive to the diaphragm in patients with severe COPD. Am J Respir Crit Care Med, 1997. 155(4): p. 1335-40.

164. Sinderby, C., et al., Diaphragm activation during exercise in chronic obstructive pulmonary disease. Am J Respir Crit Care Med, 2001. 163(7): p. 1637-41.

165. O'Donnell, D.E., et al., Qualitative aspects of exertional breathlessness in chronic airflow limitation: pathophysiologic mechanisms. Am J Respir Crit Care Med, 1997. 155(1): p. 109-15.

166. Barbera, J.A., et al., Mechanisms of worsening gas exchange during acute exacerbations of chronic obstructive pulmonary disease. Eur Respir J, 1997. 10(6): p. 1285-91.

167. Pepe, P.E. and J.J. Marini, Occult positive end-expiratory pressure in mechanically ventilated patients with airflow obstruction: the auto-PEEP effect. Am Rev Respir Dis, 1982. 126(1): p. 166-70.

168. Light, R.W., et al., Hemodynamics of patients with severe chronic obstructive pulmonary disease during progressive upright exercise. Am Rev Respir Dis, 1984. 130(3): p. 391-5.

169. Magee, F., et al., Pulmonary vascular structure and function in chronic obstructive pulmonary disease. Thorax, 1988. 43(3): p. 183-9.

170. Agusti, A.G., et al., Hypoxic pulmonary vasoconstriction and gas exchange during exercise in chronic obstructive pulmonary disease. Chest, 1990. 97(2): p. 268-75.

171. Mahler, D.A., et al., Right ventricular performance and central circulatory hemodynamics during upright exercise in patients with chronic obstructive pulmonary disease. Am Rev Respir Dis, 1984. 130(5): p. 722-9.

172. Scharf, S.M., et al., Hemodynamic characterization of patients with severe emphysema. Am J Respir Crit Care Med, 2002. 166(3): p. 314-22. 
173. Vizza, C.D., et al., Right and left ventricular dysfunction in patients with severe pulmonary disease. Chest, 1998. 113(3): p. 576-83.

174. Lopez-Campos, J.L. and A. Agusti, Heterogeneity of chronic obstructive pulmonary disease exacerbations: a two-axes classification proposal. Lancet Respir Med, 2015. 3(9): p. 729-734.

175. van Geffen, W.H., D.J. Slebos, and H.A. Kerstjens, Hyperinflation in COPD exacerbations. Lancet Respir Med, 2015. 3(12): p. e43-4.

176. van Geffen, W.H. and H.A. Kerstjens, Static and dynamic hyperinflation during severe acute exacerbations of chronic obstructive pulmonary disease. Int J Chron Obstruct Pulmon Dis, 2018. 13: p. 1269-1277.

177. Farber, M.O., et al., Studies on plasma vasopressin and the renin-angiotensin-aldosterone system in chronic obstructive lung disease. J Lab Clin Med, 1977. 90(2): p. 373-80.

178. Farber, M.O., et al., Abnormalities of sodium and $\mathrm{H} 2 \mathrm{O}$ handling in chronic obstructive lung disease. Arch Intern Med, 1982. 142(7): p. 1326-30.

179. Farber, M.O., et al., Hormonal abnormalities affecting sodium and water balance in acute respiratory failure due to chronic obstructive lung disease. Chest, 1984. 85(1): p. 49-54.

180. Stewart, A.G., et al., Changes in atrial natriuretic peptide concentrations during intravenous saline infusion in hypoxic cor pulmonale. Thorax, 1991. 46(11): p. 829-34.

181. Mannix, E.T., et al., The effect of oxygen on sodium excretion in hypoxemic patients with chronic obstructive lung disease. Chest, 1990. 97(4): p. 840-4.

182. Sharkey, R.A., et al., Renal functional reserve in patients with severe chronic obstructive pulmonary disease. Thorax, 1997. 52(5): p. 411-5.

183. Palange, P., Renal and hormonal abnormalities in chronic obstructive pulmonary disease (COPD). Thorax, 1998. 53(11): p. 989-91.

184. Baudouin, S.V., et al., Short term effect of oxygen on renal haemodynamics in patients with hypoxaemic chronic obstructive airways disease. Thorax, 1992. 47(7): p. 550-4.

185. Sharkey, R.A., E.M. Mulloy, and S.J. O'Neill, The acute effects of oxygen and carbon dioxide on renal vascular resistance in patients with an acute exacerbation of COPD. Chest, 1999. 115(6): p. 1588-92.

186. Howes, T.Q., et al., Effect of L-arginine on renal blood flow in normal subjects and patients with hypoxic chronic obstructive pulmonary disease. Thorax, 1996. 51(5): p. 516-9.

187. Schalekamp, M.A., et al., Studies on the mechanism of hypernatriuresis in essential hypertension in relation to measurements of plasma renin concentration, body fluid compartments and renal function. Clin Sci, 1971. 41(3): p. 219-31.

188. McDonald, V.M., C.R. Osadnik, and P.G. Gibson, Treatable traits in acute exacerbations of chronic airway diseases. Chron Respir Dis, 2019. 16: p. 1479973119867954.

189. Bahadori, K. and J.M. FitzGerald, Risk factors of hospitalization and readmission of patients with COPD exacerbation--systematic review. Int J Chron Obstruct Pulmon Dis, 2007. 2(3): p. 241-51.

190. Kessler, R., et al., Predictive factors of hospitalization for acute exacerbation in a series of 64 patients with chronic obstructive pulmonary disease. Am J Respir Crit Care Med, 1999. 159(1): p. 158-64.

191. Lau, A.C., L.Y. Yam, and E. Poon, Hospital re-admission in patients with acute exacerbation of chronic obstructive pulmonary disease. Respir Med, 2001. 95(11): p. 876-84.

192. Garcia-Aymerich, J., et al., Risk factors for hospitalization for a chronic obstructive pulmonary disease exacerbation. EFRAM study. Am J Respir Crit Care Med, 2001. 164(6): p. 1002-7.

193. Garcia-Sanz, M.T., et al., Factors associated with hospital admission in patients reaching the emergency department with COPD exacerbation. Multidiscip Respir Med, 2012. 7(1): p. 6.

194. Montserrat-Capdevila, J., et al., Predictive Model of Hospital Admission for COPD Exacerbation. Respir Care, 2015. 60(9): p. 1288-94.

195. Kristensen, S.R., M. Bech, and W. Quentin, A roadmap for comparing readmission policies with application to Denmark, England, Germany and the United States. Health Policy, 2015. 119(3): p. 26473.

196. Jencks, S.F., M.V. Williams, and E.A. Coleman, Rehospitalizations among patients in the Medicare feefor-service program. N Engl J Med, 2009. 360(14): p. 1418-28. 
197. Shah, T., et al., Understanding why patients with COPD get readmitted: a large national study to delineate the Medicare population for the readmissions penalty expansion. Chest, 2015. 147(5): p. 1219-1226.

198. Jo, Y.S., et al., Risk factors for early readmission after acute exacerbation of chronic obstructive pulmonary disease. Ther Adv Respir Dis, 2020. 14: p. 1753466620961688.

199. Jiang, X., et al., Trends in Readmission Rates, Hospital Charges, and Mortality for Patients With Chronic Obstructive Pulmonary Disease (COPD) in Florida From 2009 to 2014. Clin Ther, 2018. 40(4): p. 613-626 e1.

200. Sharif, R., et al., Predictors of early readmission among patients 40 to 64 years of age hospitalized for chronic obstructive pulmonary disease. Ann Am Thorac Soc, 2014. 11(5): p. 685-94.

201. Wong, A.W., et al., Acute exacerbation of chronic obstructive pulmonary disease: influence of social factors in determining length of hospital stay and readmission rates. Can Respir J, 2008. 15(7): p. 361-4.

202. Fried, L.P., et al., Frailty in older adults: evidence for a phenotype. J Gerontol A Biol Sci Med Sci, 2001. 56(3): p. M146-56.

203. McNallan, S.M., et al., Frailty and healthcare utilization among patients with heart failure in the community. JACC Heart Fail, 2013. 1(2): p. 135-41.

204. Echevarria, C., et al., The PEARL score predicts 90-day readmission or death after hospitalisation for acute exacerbation of COPD. Thorax, 2017. 72(8): p. 686-693.

205. Kong, C.W. and T.M.A. Wilkinson, Predicting and preventing hospital readmission for exacerbations of COPD. ERJ Open Res, 2020. 6(2).

206. Shah, T., et al., COPD Readmissions: Addressing COPD in the Era of Value-based Health Care. Chest, 2016. 150(4): p. 916-926.

207. Calverley, P.M., Respiratory failure in chronic obstructive pulmonary disease. Eur Respir J Suppl, 2003. 47: p. 26s-30s.

208. Plant, P.K., J.L. Owen, and M.W. Elliott, One year period prevalence study of respiratory acidosis in acute exacerbations of COPD: implications for the provision of non-invasive ventilation and oxygen administration. Thorax, 2000. 55(7): p. 550-4.

209. Roberts, C.M., et al., Acidosis, non-invasive ventilation and mortality in hospitalised COPD exacerbations. Thorax, 2011. 66(1): p. 43-8.

210. Rochwerg, B., et al., Official ERS/ATS clinical practice guidelines: noninvasive ventilation for acute respiratory failure. Eur Respir J, 2017. 50(2).

211. Mas, A. and J. Masip, Noninvasive ventilation in acute respiratory failure. Int J Chron Obstruct Pulmon Dis, 2014. 9: p. 837-52.

212. Davidson, A.C., et al., BTS/ICS guideline for the ventilatory management of acute hypercapnic respiratory failure in adults. Thorax, 2016. 71 Suppl 2: p. ii1-35.

213. Crisafulli, E., et al., Management of severe acute exacerbations of COPD: an updated narrative review. Multidiscip Respir Med, 2018. 13: p. 36.

214. Plant, P.K., J.L. Owen, and M.W. Elliott, Non-invasive ventilation in acute exacerbations of chronic obstructive pulmonary disease: long term survival and predictors of in-hospital outcome. Thorax, 2001. 56(9): p. 708-12.

215. Ambrosino, N., et al., Non-invasive mechanical ventilation in acute respiratory failure due to chronic obstructive pulmonary disease: correlates for success. Thorax, 1995. 50(7): p. 755-7.

216. Confalonieri, M., et al., A chart of failure risk for noninvasive ventilation in patients with COPD exacerbation. Eur Respir J, 2005. 25(2): p. 348-55.

217. Longhini, F., et al., Efficacy of ventilator waveform observation for detection of patient-ventilator asynchrony during NIV: a multicentre study. ERJ Open Res, 2017. 3(4).

218. Bello, G., G. De Pascale, and M. Antonelli, Noninvasive ventilation: practical advice. Curr Opin Crit Care, 2013. 19(1): p. 1-8.

219. Prasad, S.B., D. Chaudhry, and R. Khanna, Role of noninvasive ventilation in weaning from mechanical ventilation in patients of chronic obstructive pulmonary disease: an Indian experience. Indian J Crit Care Med, 2009. 13(4): p. 207-12. 
220. Lun, C.T., et al., A pilot randomized study comparing two methods of non-invasive ventilation withdrawal after acute respiratory failure in chronic obstructive pulmonary disease. Respirology, 2013. 18(5): p. 814-9.

221. Jolliet, P., et al., A Multicenter Randomized Trial Assessing the Efficacy of Helium/Oxygen in Severe Exacerbations of Chronic Obstructive Pulmonary Disease. Am J Respir Crit Care Med, 2017. 195(7): p. 871-880.

222. Titlestad, I.L., A.T. Lassen, and J. Vestbo, Long-term survival for COPD patients receiving noninvasive ventilation for acute respiratory failure. Int J Chron Obstruct Pulmon Dis, 2013. 8: p. 215-9.

223. Chu, C.M., et al., Readmission rates and life threatening events in COPD survivors treated with noninvasive ventilation for acute hypercapnic respiratory failure. Thorax, 2004. 59(12): p. 1020-5.

224. Niewoehner, D.E., The impact of severe exacerbations on quality of life and the clinical course of chronic obstructive pulmonary disease. Am J Med, 2006. 119(10 Suppl 1): p. 38-45.

225. Suissa, S., S. Dell'Aniello, and P. Ernst, Long-term natural history of chronic obstructive pulmonary disease: severe exacerbations and mortality. Thorax, 2012. 67(11): p. 957-63.

226. McGhan, R., et al., Predictors of rehospitalization and death after a severe exacerbation of COPD. Chest, 2007. 132(6): p. 1748-55.

227. Chung, L.P., et al., Five-year outcome in COPD patients after their first episode of acute exacerbation treated with non-invasive ventilation. Respirology, 2010. 15(7): p. 1084-91.

228. Funk, G.C., et al., Long-term non-invasive ventilation in COPD after acute-on-chronic respiratory failure. Respir Med, 2011. 105(3): p. 427-34.

229. Cheung, A.P., et al., A pilot trial of non-invasive home ventilation after acidotic respiratory failure in chronic obstructive pulmonary disease. Int J Tuberc Lung Dis, 2010. 14(5): p. 642-9. 


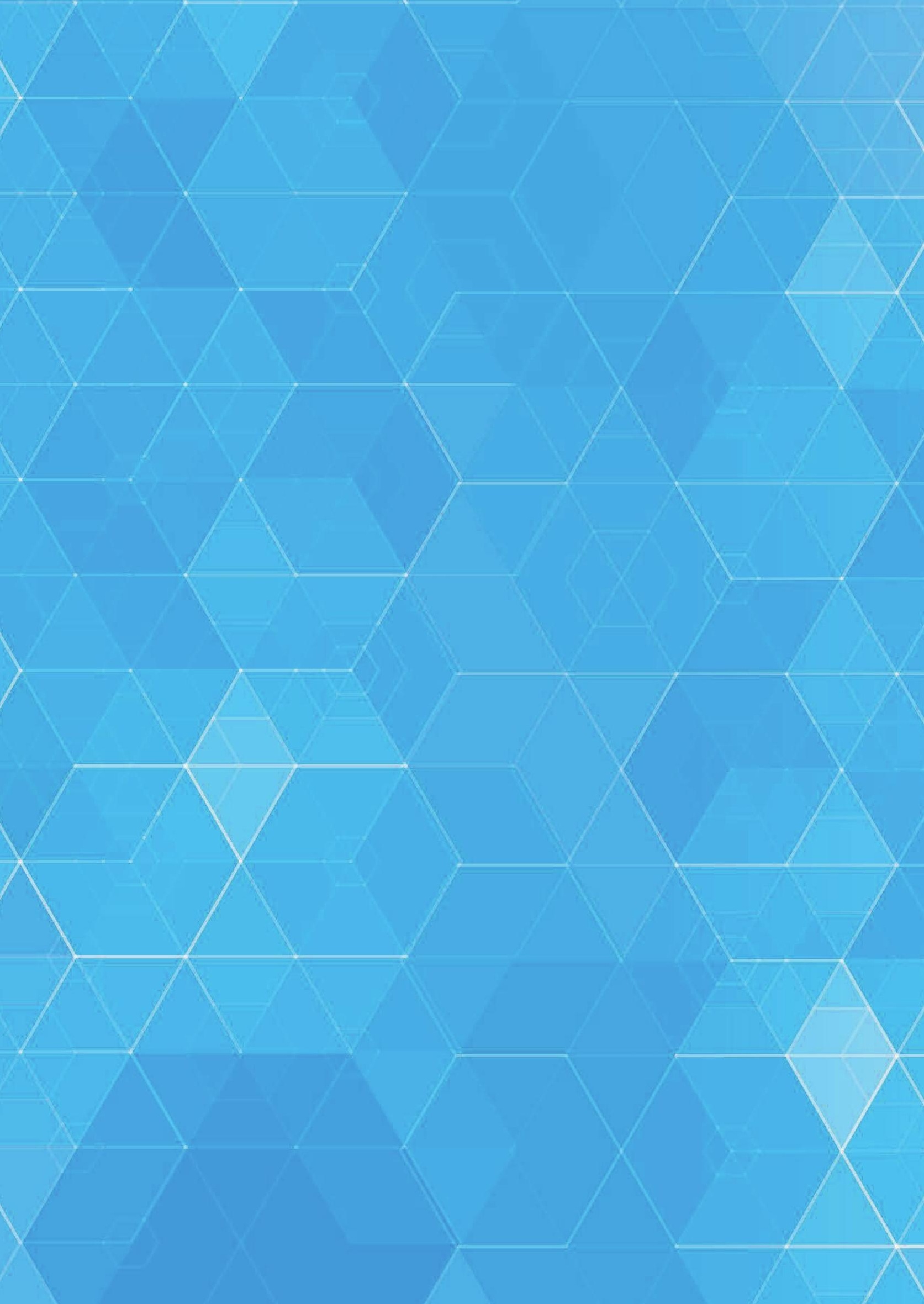




\section{Chapter 2}

Aims and outline of the thesis 



\section{Aims and outline of the thesis}

In order to improve management and outcomes of hospital based exacerbations of chronic obstructive pulmonary disease (COPD) a first part of this thesis focuses on short- and long-term mortality rates as well as on identification of predictive parameters for mortality. These findings are reported in chapters 3-6:

Chapter 3 investigated 15-year survival following hospitalization for exacerbation of COPD (ECOPD) in comparison to the general population.

Chapter 4 describes the results of a retrospective, observational study assessing the mortality rate as well as potential determinants of mortality.

Chapter 5 describes the outcomes of an easy, feasible and comprehensive predictive algorithm stratification using classification and regression trees algorithm (CART) analysis on 90 day mortality.

Chapter 6 investigates the response on non-invasive ventilation (NIV) as a predictor for mortality in patients hospitalized for ECOPD accompanied with acute respiratory failure.

The effectiveness of non-invasive ventilation in COPD patients with prolonged hypercapnia after ventilator support for acute respiratory failure is still unclear. Chapter $\mathbf{7}$ describes the results of the RESCUE study investigating if nocturnal NIV in these patients prolongs the time to readmission for respiratory causes or death in a one-year follow up period.

In a second part of this thesis we will explore the effects of blood gas disturbances on the functional integrity of the gastro-intestinal tract, a crucial organ in the transport of fluid, nutrients and electrolytes to and from the intestinal lumen. This epithelium exists at the interface of the richly vascularized mucosa and the anoxic luminal environment. ${ }^{1}$ The intestinal epithelium separates the human body from the harsh and changeable environment of the intestinal lumen. Therefore, the mucosal epithelial integrity has a pivotal role in preventing the entry of potentially harmful luminal particles, e.g. bacteria and their products into systemic blood compartment through trans-cellular and paracellular pathways. ${ }^{2}$ Disturbed intestinal integrity might result to an increased permeability of the intestinal mucosal barrier. This is one of the mechanisms enhancing bacterial translocation, which could potentially contribute to acute chronic flare-ups of systemic inflammation reported in severe ECOPD. ${ }^{3-6}$ 
To maintain adequate oxygen $\left(\mathrm{O}_{2}\right)$ availability in the intestinal epithelium, intestinal vascular flow regulation and particularly at the villi, is very crucial. The splanchnic blood flow must be considered in relation to the splanchnic metabolic activity and the oxygen extraction capabilities of the splanchnic tissues. Normally, the total hepato-splanchnic blood flow is approximately $20-30 \%$ of cardiac output. The splanchnic oxygen consumption is about $20-35 \%$ of the whole body oxygen consumption. ${ }^{7}$ Regulation of blood volume and blood flow in the splanchnic region is a crucial regulator of this metabolic homeostasis: an increase of about $50-100 \%$ has been reported in the celiac and superior mesenteric artery flow following meal ingestion. ${ }^{8}$ Incretins as glucosedependent insulin-trophic polypeptide (GIP) and glucagon-like peptide-1 (GLP-1) play an important role in the blood flow redistribution in the splanchnic vascular bed. ${ }^{9}$ The blood flow of the small intestinal villus is very particular: the artery and vein run in parallel but their blood flows are in opposite directions. This anatomical arrangement allows countercurrent exchange of oxygen from the artery to the vein along their course within the villus, resulting in a descending gradient of tissue $\mathrm{PO}_{2}$ from the base of the villus to its tip. ${ }^{10,11}$ This mechanism may make the villus susceptible to tissue hypoxia. $^{7}$

The splanchnic region has a large capacity to adapt to reducing blood flow by increasing the oxygen extraction but particularly the gut is more prone to develop hypoxia as has been found in experimental studies where the gut may become hypoxic when its oxygen extraction approaches $70 \% .^{7,12,13}$ It can be hypothesized that besides modulation of blood flow, oxygen content of arterial blood is another important factor for villus oxygenation. Interestingly, it has been demonstrated that hypercapnia improves oxygen delivery and microvascular oxygenation of the gastric mucosa not only during physiological conditions, but also during hemorrhagic shock. ${ }^{14,15}$ However, these positive effects on microcirculatory oxygenation might be partially counterbalanced by an increase in vasopressin levels, induced by hypercapnia. ${ }^{16,17}$ Vasopressin is regarded as a potent vasoconstrictor particularly in the splanchnic region and reduces jejunal mucosal oxygen partial pressure. ${ }^{18,19}$ Vasopressin V1a receptors (V1AR) seem to mediate hypercapnic increase in gastric mucosal oxygenation. ${ }^{20}$ In general, these findings illustrate the need to consider the effects of oxygenation and hypercapnia on gut integrity at least in patients with respiratory failure.

The emerging role of the gut in chronic diseases, including chronic heart failure (CHF), another multicomponent chronic organ disease characterized by a combination of increased sympathetic tone, anabolic/catabolic dysbalance and chronic inflammation. ${ }^{21}$ Even in stable, compensated $\mathrm{CHF}$ patients an increased intestinal para-cellular 
permeability is reported. ${ }^{22,23}$ In patients with acute heart failure or cardiogenic shock, disturbances of intestinal integrity, manifested by increased intestinal fatty acid binding protein (I-FABP) levels are associated with higher mortality, suggesting that inadequate perfusion of the small intestine might have an pivotal role in the worse outcome. ${ }^{24}$ In stable COPD patients, Rutten et al. reported significantly elevated serum I-FABP levels measured after performing daily life activities ${ }^{25}$ : redistribution of blood flow from the splanchnic region to the exercising muscles could be a contributing factor in this temporary increase in I-FABP levels. ${ }^{26}$

The role and potential impact of gut dysintegrity in relation to ECOPD is studied in chapters 8 and 9.

Chapter $\mathbf{8}$ explores the impact of severe COPD on the gastro-intestinal permeability in patients admitted with ECOPD and hypoxemia at admission and after 4 weeks recovery.

Chapter 9 a study which was aimed to provide more insight of the association between gastro-intestinal integrity, systemic inflammation and risk of exacerbation.

In Chapter $\mathbf{1 0}$ the results of the previous chapters in the current dissertation are discussed and future perspectives for research in this area are suggested in order to improve the burden and outcomes of these life-threatening events in the lives of COPD patients. 


\section{References}

1. Ward, J.B., S.J. Keely, and S.J. Keely, Oxygen in the regulation of intestinal epithelial transport. J Physiol, 2014. 592(12): p. 2473-89.

2. Chelakkot, C., J. Ghim, and S.H. Ryu, Mechanisms regulating intestinal barrier integrity and its pathological implications. Exp Mol Med, 2018. 50(8): p. 103.

3. van Eeden, S.F. and D.D. Sin, Chronic obstructive pulmonary disease: a chronic systemic inflammatory disease. Respiration, 2008. 75(2): p. 224-38.

4. Bjarnason, I., A. MacPherson, and D. Hollander, Intestinal permeability: an overview. Gastroenterology, 1995. 108(5): p. 1566-81.

5. Berg, R.D., Bacterial translocation from the gastrointestinal tract. Adv Exp Med Biol, 1999. 473: p. 11-30.

6. Turner, J.R., Intestinal mucosal barrier function in health and disease. Nat Rev Immunol, 2009. 9(11): p. 799-809.

7. Takala, J., Determinants of splanchnic blood flow. Br J Anaesth, 1996. 77(1): p. 50-8.

8. Someya, N., et al., Blood flow responses in celiac and superior mesenteric arteries in the initial phase of digestion. Am J Physiol Regul Integr Comp Physiol, 2008. 294(6): p. R1790-6.

9. Koffert, J., et al., Effects of meal and incretins in the regulation of splanchnic blood flow. Endocr Connect, 2017. 6(3): p. 179-187.

10. Lundgren, O. and U. Haglund, The pathophysiology of the intestinal countercurrent exchanger. Life Sci, 1978. 23(14): p. 1411-22.

11. Geboes, K., K.P. Geboes, and G. Maleux, Vascular anatomy of the gastrointestinal tract. Best Pract Res Clin Gastroenterol, 2001. 15(1): p. 1-14.

12. Nelson, D.P., et al., Pathological supply dependence of systemic and intestinal $\mathrm{O} 2$ uptake during endotoxemia. J Appl Physiol (1985), 1988. 64(6): p. 2410-9.

13. Zhang, H., et al., Tirilazad mesylate (U-74006F) inhibits effects of endotoxin in dogs. Am J Physiol, 1995. 268(5 Pt 2): p. H1847-55.

14. Schwartges, I., et al., Hypercapnia induces a concentration-dependent increase in gastric mucosal oxygenation in dogs. Intensive Care Med, 2008. 34(10): p. 1898-906.

15. Schwartges, I., et al., Hypercapnic acidosis preserves gastric mucosal microvascular oxygen saturation in a canine model of hemorrhage. Shock, 2010. 34(6): p. 636-42.

16. Farber, M.O., et al., Abnormalities of sodium and $\mathrm{H} 2 \mathrm{O}$ handling in chronic obstructive lung disease. Arch Intern Med, 1982. 142(7): p. 1326-30.

17. Rose, C.E., Jr., R.J. Anderson, and R.M. Carey, Antidiuresis and vasopressin release with hypoxemia and hypercapnia in conscious dogs. Am J Physiol, 1984. 247(1 Pt 2): p. R127-34.

18. Knotzer, H., et al., Arginine vasopressin reduces intestinal oxygen supply and mucosal tissue oxygen tension. Am J Physiol Heart Circ Physiol, 2005. 289(1): p. H168-73.

19. Knotzer, H., et al., Arginine vasopressin does not alter mucosal tissue oxygen tension and oxygen supply in an acute endotoxemic pig model. Intensive Care Med, 2006. 32(1): p. 170-4.

20. Vollmer, C., et al., Vasopressin $\mathrm{V}(1 \mathrm{~A})$ receptors mediate the increase in gastric mucosal oxygenation during hypercapnia. J Endocrinol, 2013. 217(1): p. 59-67.

21. Peschel, T., et al., Invasive assessment of bacterial endotoxin and inflammatory cytokines in patients with acute heart failure. Eur J Heart Fail, 2003. 5(5): p. 609-14.

22. Teixeira, T.F., et al., Intestinal permeability parameters in obese patients are correlated with metabolic syndrome risk factors. Clin Nutr, 2012. 31(5): p. 735-40.

23. Sandek, A., et al., Altered intestinal function in patients with chronic heart failure. J Am Coll Cardiol, 2007. 50(16): p. 1561-9.

24. Kastl, S.P., et al., Intestinal Fatty Acid Binding Protein is Associated With Mortality in Patients With Acute Heart Failure or Cardiogenic Shock. Shock, 2019. 51(4): p. 410-415.

25. Rutten, E.P.A., et al., Disturbed intestinal integrity in patients with COPD: effects of activities of daily living. Chest, 2014. 145(2): p. 245-252.

26. Kartaram, S., et al., Plasma citrulline concentration, a marker for intestinal functionality, reflects exercise intensity in healthy young men. Clin Nutr, 2019. 38(5): p. 2251-2258. 



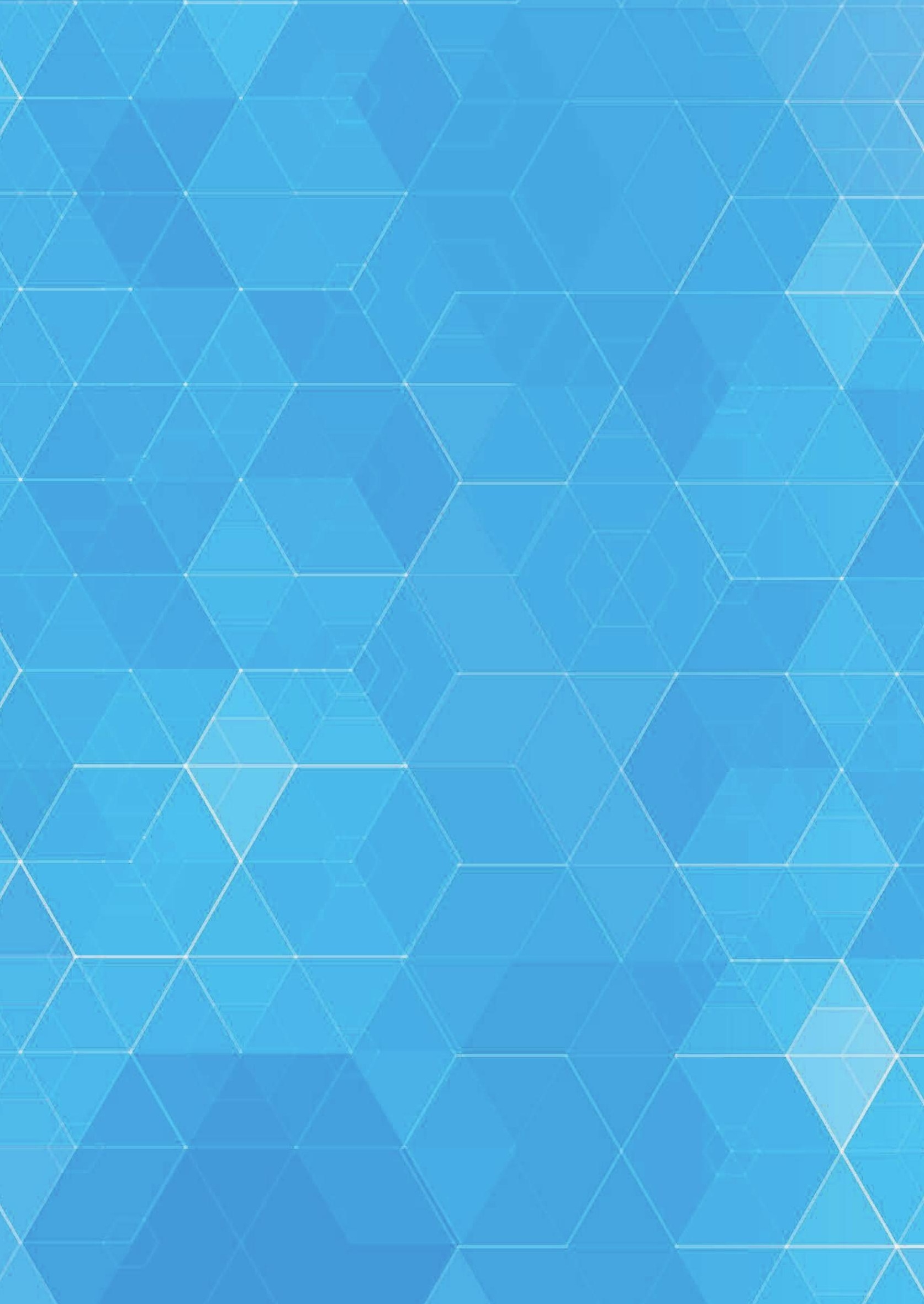




\section{Chapter 3}

\section{Long-term survival after admission for COPD exacerbation: a comparison with}

the general population

Pauline V. van Hirtum*, Roy T.M. Sprooten*, Jan A. van Noord, Monique van Vliet, Martijn D. de Kruif

* These authors contributed equally to the study Published in Respiratory Medicine 2018;137:77-82 


\section{Abstract}

\section{Background}

Life expectancy data of Chronic Obstructive Pulmonary Disease (COPD) patients in comparison to the general population are primarily based upon long-term population cohort studies. These studies are limited by a poor definition of clinically significant COPD. The key element in the course of COPD is a clinical exacerbation. Therefore, this study investigated 15-year survival following hospitalization for an exacerbation of COPD in comparison to the general population.

\section{Methods}

A number of 4229 subjects was studied, including 845 hospitalized COPD patients and 3384 age and sex matched controls. Mortality risks were assessed using Kaplan-Meier survival curves, and hazard rate ratios for death were estimated using Cox proportional hazards regression models, for each GOLD class separately.

\section{Results}

Overall 15-year survival was $7.3 \%$ in the COPD group and $40.6 \%$ in the general population. Survival was $24 \%, 11.1 \%, 5.3 \%$ and $0 \%$ for COPD GOLD I-IV. The mean life expectancy following hospitalization was 9.7, 7.1, 6.1 and 3.4 years for stage GOLD I-IV and 10.2 years for the general population. Overall, negative prognostic factors were age, male gender, low Forced Expiratory Volume in 1 second $\left(\mathrm{FEV}_{1}\right)$, low transfer factor of the lung for carbon monoxide (TLCO), respiratory insufficiency, Charlson comorbidity class, ICU-admission and exacerbation frequency. Factors differed among GOLD stages.

\section{Conclusions}

The 15 -year survival for hospitalized COPD patients is reduced by $82 \%$ in comparison to the general population. This indicates a more deleterious course of clinically significant COPD in comparison to population cohorts. As such, every possible effort should be taken to reduce exacerbations in a personalized way. 


\section{Introduction}

Chronic Obstructive Pulmonary Disease (COPD) is one of the leading causes of death worldwide. ${ }^{1}$ In addition, COPD is associated with a high morbidity and socio-economic impact. The long term mortality in COPD has been studied in several population cohorts. $^{2-6}$ The main prognostic factors in these cohort studies appeared to be age and GOLD stage.

Despite the helicopter view offered by population cohort studies, these studies remain limited by a shortage of clinical information. One needs to keep in mind that COPD represents a wide spectrum of subtypes, each of them associated with specific mortality and morbidity figures. ${ }^{7-9}$ In particular, the key element in the course of COPD is admission to the hospital due to an exacerbation. Hospital admission is not only associated with increased mortality and decline of lung function and performance status, but it also represents the mainstay of costs in COPD care. ${ }^{10}$ Therefore, many studies have investigated the short term impact of clinical exacerbations on survival and studied prognostic factors. ${ }^{8,11-14}$ However, long term survival after a clinical exacerbation remained to be determined, and no figures were available relating survival after a clinical exacerbation with the general population.

Therefore, this study aimed to determine long-term survival in a cohort of COPD patients following a clinical exacerbation, in comparison to survival in age and sex matched members of the general population from the same region. In addition, to address the diversity of COPD patients, this study investigated predictors of mortality per GOLD class separately.

\section{Methods}

\section{Study population}

The study was performed at the Zuyderland Medical Center (location Heerlen, The Netherlands), which is a large teaching hospital in a former coal mining area in the southeast part of the province of Limburg, The Netherlands. Patients were retrospectively included between January $1^{\text {st }} 1996$ and September $15^{\text {th }} 1999$. All subjects were admitted to the hospital with a clinical diagnosis of COPD exacerbation. COPD was diagnosed by a physician based upon clinical symptoms, evidence of airway obstruction (ratio Forced Expiratory Volume in one second $\left(\mathrm{FEV}_{1}\right)$ /Vital Capacity (VC) $<70 \%$ ) and previous exposure to noxious gasses. Patients with asthma were excluded based upon extensive chart review. An exacerbation was defined as an increase in 
respiratory symptoms like dyspnoea, cough, and/or increased sputum production. All patients received standard care according to international guidelines. ${ }^{15}$ The study design was approved by the ethics committee of the Zuyderland Hospital (approval number 16N164).

Clinical data were obtained from patient records: age, sex, gender, Body Mass Index (BMI), Forced Expiratory Volume in 1 second $\left(\mathrm{FEV}_{1}\right.$, expressed as percent predicted according to EGKS normal values), transfer factor of the lung for carbon monoxide (TLCO), chronic respiratory insufficiency, Charlson comorbidity index, smoking status, mechanical ventilation at the intensive care unit (ICU), a history revealing any previous admission within a year from inclusion and the mean number of admissions per year in the first five years after inclusion. GOLD stage was defined according to Global Initiative for Chronic Obstructive Lung Disease (GOLD). Chronic respiratory insufficiency was defined by either chronic hypoxemia $\left(\mathrm{PaO}_{2}<8.0 \mathrm{kPa}\right)$ or chronic hypercapnia $\left(\mathrm{PaCO}_{2} \geq 6.0 \mathrm{kPa}\right)$ in stable condition, either measured at the end of hospital stay or at the outpatient clinic. Patients were considered smoker if they had $>10$ pack years and ex-smoker if they quit smoking for $>6$ months. All variables except for re-admission rates and chronic hypercapnia data were determined at the index clinical exacerbation.

The date and cause of death (COPD, cardiovascular, lung cancer or other) were collected from the patient records and/or from information obtained by contacting the general practitioner by phone. When no information was available, the last date was recorded when the patient had visited the hospital or general practitioner. For survival analysis, these data were censored.

\section{General population}

For each study patient, four age and sex matched controls were collected at random from the general population in the southeast part of the province of Limburg, The Netherlands, by the Dutch National Statistics Office (Centraal Bureau voor de Statistiek, Heerlen, The Netherlands). Both the year of birth and the year of inclusion were required to be the same for patients as well as their matched subjects and the subjects had to be alive at the time of inclusion. For all subjects, gender, age and survival data were registered.

\section{Statistics}

Analysis was performed using SPSS version 20.0 Patient characteristics were compared per GOLD class using One-Way ANOVA. Survival was estimated with Kaplan-Meier 
survival plots with log-rank, Breslow and Tarone-Ware testing. Mean life expectancy \pm SE was derived from Kaplan-Meier analysis. Annual mortality rate and excess death rate (EDR) were calculated from survival data similar to the method described by Shavelle. ${ }^{6}$ Both bivariate and multivariate Cox regression analyses were performed to estimate the effect of potential covariates on survival. A p-value $<0.05$ was considered statistically significant.

\section{Results}

\section{Subjects}

A total of 4229 subjects was studied, including 845 COPD patients and 3384 age and sex matched subjects from the general population. The clinical characteristics of the COPD patients are shown in Table 3.1. Distribution of GOLD stage I to IV was $8.9 \%, 34.2 \%$, $26.5 \%, 30.5 \%$, respectively. For each increasing GOLD stage, a decreasing FEV $_{1}$ and TLCO was observed, as well as an increasing proportion of males and slightly decreasing BMI levels.

Table 3.1 Patient characteristics. Data are presented as numbers with percentages or means with standard deviation. p-values were calculated by One-Way ANOVA for differences among GOLD classes.

\begin{tabular}{|c|c|c|c|c|c|c|}
\hline Characteristics & $\begin{array}{c}\text { All patients } \\
\mathrm{n}=\mathbf{8 4 5}\end{array}$ & $\begin{array}{c}\text { GOLD I } \\
n=75\end{array}$ & $\begin{array}{c}\text { GOLD II } \\
n=288\end{array}$ & $\begin{array}{c}\text { GOLD III } \\
n=224\end{array}$ & $\begin{array}{c}\text { GOLD IV } \\
n=258\end{array}$ & $\begin{array}{c}\mathrm{p}- \\
\text { value }\end{array}$ \\
\hline Age at admission (y) & $70( \pm 10)$ & $69( \pm 12)$ & $70( \pm 10)$ & $69( \pm 10)$ & $70( \pm 8)$ & 0.406 \\
\hline Gender (male) & $490(58 \%)$ & $29(38.7 \%)$ & $156(54.2 \%)$ & $137(61.2 \%)$ & $168(65.1 \%)$ & 0.001 \\
\hline BMI $\left(\mathrm{kg} / \mathrm{m}^{2}\right)$ & $24.8( \pm 5.8)$ & $26.2( \pm 5.9)$ & $26.1( \pm 6.7)$ & $24.0( \pm 4.7)$ & $23.6( \pm 5.3)$ & $<0.001$ \\
\hline Underweight & $78(9.2 \%)$ & $3(4 \%)$ & $18(6.3 \%)$ & $22(9.8 \%)$ & 35 (13.6\%) & 0.001 \\
\hline \multicolumn{7}{|l|}{ Smoking status } \\
\hline current & $360(42.6 \%)$ & 25 (33\%) & $134(46.5 \%)$ & $101(45.1 \%)$ & 99 (38.5\%) & $<0.001$ \\
\hline ex-smoker & $459(54.3 \%)$ & 47 (62.7\%) & $146(50.7 \%)$ & $119(53.1 \%)$ & $147(57.2 \%)$ & $<0.001$ \\
\hline unknown & $24(2.8 \%)$ & $3(4 \%)$ & $8(2.8 \%)$ & $4(1.7 \%)$ & $11(4.3 \%)$ & $<0.001$ \\
\hline $\mathrm{FEV}_{1}$ (\%predicted) & $49( \pm 18)$ & $81( \pm 7)$ & $62( \pm 9)$ & $40( \pm 6)$ & $33( \pm 11)$ & $<0.001$ \\
\hline TLCO (\%predicted) & $54( \pm 22)$ & $74( \pm 21)$ & $61( \pm 20)$ & $51( \pm 20)$ & $42( \pm 17)$ & $<0.001$ \\
\hline CRF & $179(21.2 \%)$ & 0 & 0 & 0 & $179(69.4 \%)$ & $<0.001$ \\
\hline $\mathrm{CCl}$ & $2.9( \pm 1.5)$ & $2.7( \pm 1.5)$ & $3.0( \pm 1.6)$ & $2.8( \pm 1.5)$ & $2.8( \pm 1.5)$ & 0.275 \\
\hline ICU mechanical ventilation & $34(4 \%)$ & 0 & $7(2.4 \%)$ & $12(5.4 \%)$ & $15(5.8 \%)$ & 0.042 \\
\hline Previous admission $<1 \mathrm{y}$ & $523(61.9 \%)$ & $58(77.3 \%)$ & $231(80.2 \%)$ & $171(76.3 \%)$ & $163(63 \%)$ & $<0.001$ \\
\hline Admissions/y (<5 y) & $1.1( \pm 1.0)$ & $0.5( \pm 0.5)$ & $0.9( \pm 0.9)$ & $1.1( \pm 1.2)$ & $1.3( \pm 0.9)$ & $<0.001$ \\
\hline
\end{tabular}

Data are presented as $\mathrm{n}(\%)$ or mean \pm SD. Abbreviations: y: year, BMI: Body mass index, FEV ${ }_{1}$ : forced expiratory volume in 1 second, TLCO: diffusion capacity of carbon monoxide, CRF: chronic respiratory failure, $\mathrm{CCl}$ : Charlson comorbidity index, ICU: intensive care unit. 


\section{Survival}

The 5, 10 and 15-year survival of COPD patients following a clinical exacerbation was $43.7 \%, 19.9 \%$ and $7.3 \%$, respectively (Table 3.2 ). In the general population, survival was $76.9 \%, 57.2 \%$ and $40.6 \%$, respectively. The 15 -year survival per GOLD stage I-IV was $24 \%, 11.1 \%, 5.3 \%$ and $0 \%$, respectively (Figure 3.1). All stages except for GOLD I differed significantly from the general population $(p<0.000)$. Overall, the GOLD I group was not significantly different, but interval analysis demonstrated increased mortality after 5 years $(p=0.011)$.

Table 3.2 Survival data and cause of death. Censored cases represent cases lost to follow up during the observation period.

\begin{tabular}{|c|c|c|c|c|c|}
\hline Survival & $\begin{array}{c}\text { All patients } \\
n=845\end{array}$ & $\begin{array}{c}\begin{array}{c}\text { COPD GOLD I } \\
\mathrm{n}=75\end{array} \\
\end{array}$ & $\begin{array}{c}\text { COPD GOLD II } \\
\mathrm{n}=\mathbf{2 8 8}\end{array}$ & $\begin{array}{c}\text { COPD GOLD III } \\
n=224\end{array}$ & $\begin{array}{c}\text { COPD GOLD IV } \\
\mathrm{n}=\mathbf{2 5 8} \\
\end{array}$ \\
\hline \multicolumn{6}{|l|}{ Survival $<5$ y } \\
\hline Survivors & 369 (43.7\%) & 55 (73.3\%) & 151 (52.4\%) & 106 (47.3\%) & 57 (22.0\%) \\
\hline Non survivors & 441 (52.2\%) & $14(18.7 \%)$ & $126(43.8 \%)$ & 109 (48.7\%) & $192(74.4 \%)$ \\
\hline Censored & 35 (4.1\%) & $6(8 \%)$ & $11(3.8 \%)$ & $9(4 \%)$ & $9(3.4 \%)$ \\
\hline \multicolumn{6}{|l|}{ Survival <10 y } \\
\hline Survivors & 168 (19.9\%) & $33(44 \%)$ & $78(27.1 \%)$ & $42(18.8 \%)$ & 15 (5.8\%) \\
\hline Non survivors & $612(72.4 \%)$ & 32 (42.7\%) & $182(63.2 \%)$ & 165 (73.7\%) & 233 (90.3\%) \\
\hline Censored & $65(7.7 \%)$ & $10(13.3 \%)$ & $28(9.7 \%)$ & 17 (7.5\%) & $10(3.9 \%)$ \\
\hline \multicolumn{6}{|l|}{ Survival <15 y } \\
\hline Survivors & $62(7.3 \%)$ & $18(24 \%)$ & 32 (11.1\%) & $12(5.3 \%)$ & 0 \\
\hline Non survivors & 705 (83.6\%) & 46 (61.3\%) & 222 (77.1\%) & $192(85,7 \%)$ & 245 (95.0\%) \\
\hline Censored & 78 (9.2\%) & $11(14.7 \%)$ & 34 (11.8\%) & $20(8.9 \%)$ & $13(5.0 \%)$ \\
\hline \multicolumn{6}{|l|}{ Cause of death } \\
\hline COPD & $368(43.6 \%)$ & $6(8 \%)$ & $87(30.2 \%)$ & 107 (47.8\%) & $168(65.1 \%)$ \\
\hline Cardiovascular & 119 (14.1\%) & $12(16 \%)$ & $51(17.7 \%)$ & $20(8.9 \%)$ & $36(14 \%)$ \\
\hline Lung carcinoma & $32(3.8 \%)$ & $2(2,7 \%)$ & $4(1.4 \%)$ & $20(8.9 \%)$ & $6(2.3 \%)$ \\
\hline Other & $113(13.4 \%)$ & $16(21.3 \%)$ & 47 (16.3\%) & $27(12.1 \%)$ & $23(8.9 \%)$ \\
\hline Unknown & $213(25.2 \%)$ & 39 (52.0\%) & $99(34.3 \%)$ & $50(22.3 \%)$ & $25(9.7 \%)$ \\
\hline
\end{tabular}

Data are presented as $\mathrm{n}(\%) ; \mathrm{y}$ : year.

Cause of death was COPD (54\%), cardiovascular disease (16\%), lung carcinoma (4\%) or other (18\%). Death due to COPD was $18 \%$ versus $71 \%$ among GOLD I and GOLD IV patients, respectively.

\section{Life expectancy}

The mean life expectancy following an exacerbation of COPD was 9.7, 7.1, 6.1 and 3.4 years for stage GOLD I-IV, respectively (Table 3.3). In the general population, mean survival was 10.2 years. 
Figure 3.1 15-year survival in a cohort of COPD patients after clinical exacerbation ( $n=845)$, stratified into GOLD stage I-IV, in comparison to sex and age matched subjects from general population $(n=3384)$.

\section{Survival of Gold stages}

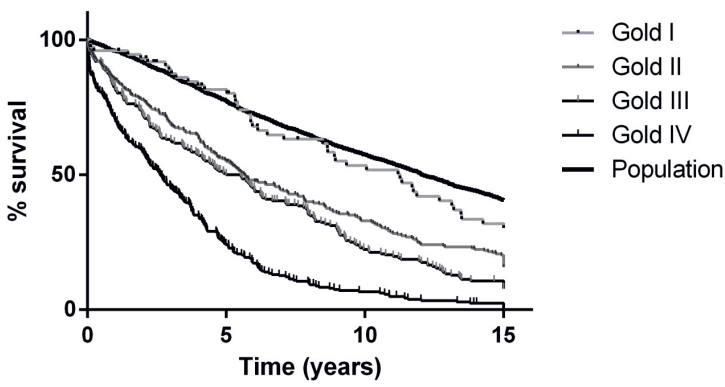

The annual mortality rate and excess death rate of the COPD group versus the general population are shown in table 3. Due to lack of survivors, data were not calculated for COPD GOLD IV after 15 years.

Table 3.3 Life expectancy. Life expectancy (LE), annual mortality rate (AMR) and excess death rate (EDR) after clinical exacerbation COPD.

\begin{tabular}{lccccc}
\hline & General population & GOLD I & GOLD II & GOLD III & GOLD IV \\
\hline LE 5 y & $12.1 \pm 0.91$ & $11.60 \pm 0.66$ & $8.86 \pm 0.39$ & $8.14 \pm 0.44$ & $4.776 \pm 0.35$ \\
LE 10 y & $10.64 \pm 0.92$ & $9.16 \pm 0.64$ & $6.83 \pm 0.33$ & $6.03 \pm 0.34$ & $3.40 \pm 0.23$ \\
LE 15 y & $10.21 \pm 0.88$ & $9.66 \pm 0.61$ & $7.11 \pm 0.33$ & $6.13 \pm 0.33$ & $3.41 \pm 0.22$ \\
AMR 5 y & 0.0525 & 0.0629 & 0.1293 & 0.1506 & 0.3010 \\
AMR 10 y & 0.0559 & 0.0821 & 0.1306 & 0.1677 & 0.2847 \\
AMR 15 y & 0.0601 & 0.0951 & 0.1465 & 0.1958 & NA \\
EDR 5 y & -3.311 & 0.0104 & 0.0767 & 0.0980 & 0.2485 \\
EDR 10 y & 0.0559 & 0.0262 & 0.0747 & 0.1118 & 0.2289 \\
EDR 15 y & 0.0601 & 0.0350 & 0.0865 & 0.1357 & NA \\
\hline
\end{tabular}

Mean life expectancy (LE) is presented in years (y), \pm SE. NA=not applicable.

\section{Prognostic factors}

In bivariate analysis, negative prognostic factors for survival after clinical exacerbation of COPD were high age, male gender, low $\mathrm{FEV}_{1}$, low TLCO, chronic respiratory insufficiency, high Charlson comorbidity index, current smoking at time of admission, $\mathrm{BMI}<18.5 \mathrm{~kg} / \mathrm{m}^{2}$, ICU-admission for mechanical ventilation, previous admission $<1$ year and mean number of admissions. 
In multivariate analysis, the strongest predictors were age, FEV ${ }_{1}$, TLCO, chronic respiratory insufficiency, Charlson comorbidity index and mean number of admissions in first five years, whereas a weaker relationship was found for gender, a trend towards significance for previous admission <1 year and ICU admission and no significant relationship was found for active smoking and being underweight.

In all GOLD classes, age and mean number of admissions in the first five years were the most important risk factors for mortality. Furthermore, in the GOLD I group, also gender and Charlson comorbidity index contributed significantly. In the GOLD II group, TLCO added further prognostic value whereas gender, ICU admission and previous admission <1 year showed a trend towards significance. In the GOLD III group, TLCO and Charlson comorbidity index also contributed to prognosis and gender and $\mathrm{FEV}_{1}$ showed a trend towards significance. In the GOLD IV group, a lower FEV $_{1}$ and chronic respiratory insufficiency increased the mortality risk further whereas a trend towards significance was observed for TLCO.

An additional multivariate analysis was performed in five-year survivors in the GOLD I group $(n=55)$. Apart from age, active smoking was the most important risk factor in this particular group ( $H R$ 3.31; $p=0.020$ ). Other risk factors were male gender (HR 2.8; $\mathrm{p}=0.018)$, Charlson comorbidity index (HR 1.53; $\mathrm{p}=0.015)$ and previous admission $<1$ year (HR 2.95; $p=0.025)$.

Finally, since BMI $<18.5 \mathrm{~kg} / \mathrm{m}^{2}$ was a relevant risk factor in bivariate, but not in multivariate analysis, confounding factors for BMI were explored. Being underweight was associated a decreased TLCO, number of readmissions and current smoking at inclusion (ODDS ratio=0.956, $p=0.000$; $O R=1.484, p=0.001$ and $p=0.041$ by Chi Square, respectively). 
Table 3.4 Prognostic factors. Prognostic factors in 15-year survival after clinical COPD exacerbation. Hazard ratio (HR) reflecting mortality risk was calculated by multivariate cox regression.

\begin{tabular}{|c|c|c|c|c|c|c|c|c|c|c|}
\hline & \multicolumn{2}{|c|}{ All COPD patients } & \multicolumn{2}{|c|}{ COPD GOLD I } & \multicolumn{2}{|c|}{ COPD GOLD II } & \multicolumn{2}{|c|}{ COPD GOLD III } & \multicolumn{2}{|c|}{ COPD GOLD IV } \\
\hline & $\mathrm{HR}$ & $p$ & $\mathrm{HR}$ & $p$ & $\mathrm{HR}$ & $p$ & $\mathrm{HR}$ & $p$ & $\mathrm{HR}$ & $p$ \\
\hline Age & 1.069 & 0.000 & 1.086 & 0.000 & 1.073 & 0.000 & 1.068 & 0.000 & 1.067 & 0.000 \\
\hline Gender (male) & 1.208 & 0.005 & 2.929 & 0.005 & 1.336 & 0.053 & 1.360 & 0.080 & 0.813 & NS \\
\hline $\mathrm{FEV}_{1}$ (\%predicted) & 0.988 & 0.000 & 1.027 & NS & 0.987 & NS & 0.976 & 0.081 & 0.978 & 0.007 \\
\hline TLCO (\%predicted) & 0.988 & 0.000 & 0.997 & NS & 0.985 & 0.000 & 0.987 & 0.009 & 0.992 & 0.087 \\
\hline BMI $\left(<18.5 \mathrm{~kg} / \mathrm{m}^{2}\right)$ & 1.119 & NS & 0.954 & NS & 1.420 & NS & 1.179 & NS & 1.001 & NS \\
\hline $\mathrm{CCl}$ & 1.125 & 0.000 & 1.681 & 0.000 & 1.060 & NS & 1.115 & 0.016 & 1.082 & NS \\
\hline Current smoking & 1.089 & NS & 2.161 & NS & 0.905 & NS & 1.124 & NS & 1.158 & NS \\
\hline CRF & 1.693 & 0.000 & NA & NA & NA & NA & NA & NA & 1.616 & 0.003 \\
\hline $\begin{array}{l}\text { ICU mechanical } \\
\text { ventilation }\end{array}$ & 1.408 & 0.099 & NA & NA & 2.402 & 0.062 & 1.247 & NS & 1.158 & NS \\
\hline $\begin{array}{l}\text { Any previous } \\
\text { admission }<1 \text { year }\end{array}$ & 1.208 & 0.052 & 1.244 & NS & 1.365 & 0.091 & 1.153 & NS & 1.149 & NS \\
\hline $\begin{array}{l}\text { Re-admissions } \\
\text { first } 5 \text { years }\end{array}$ & 1.646 & 0.000 & 7.691 & 0.000 & 1.961 & 0.000 & 1.516 & 0.000 & 1.661 & 0.000 \\
\hline
\end{tabular}

$\mathrm{NS}=$ Not Significant $(\mathrm{p}>0.100) . \mathrm{NA}=$ not applicable. $\mathrm{FEV}_{1}$ : forced expiratory volume in 1 second, TLCO: diffusion capacity of carbon monoxide, BMI: Body mass index, $\mathrm{CCl}$ : Charlson Comorbidity Index, CRF: chronic respiratory failure, ICU: Intensive care unit.

\section{Discussion}

The importance of this study is foremost that for the first time, long term life expectancy following a clinical exacerbation of COPD has been calculated and compared with the general population. The overall 15-year survival after a clinical exacerbation of COPD was only $7.3 \%$, whereas survival in the matched general population was $40.6 \%$. Since clinical exacerbations are the mainstay of costs in COPD care, these figures are relevant for physicians as well as their patients and policy makers.

When the figures from our study are compared with data from the only large population cohort COPD that presented life expectancy data before ${ }^{6}$, it shows that our death rates are increased by a factor up to 2.9 for subgroup COPD GOLD II (EDR=0.0747 vs. $E D R=0.0257$, respectively). These data support the concept that clinical exacerbations have a major, deleterious impact on the course of COPD. ${ }^{16}$ As such, one could argue that the COPD GOLD II group in particular should receive extra specialist attention following a clinical exacerbation. The EDR of the combined GOLD III and IV groups differed to a lesser extent (factor 1.5). Probably, patients with these GOLD 
stages in the population cohort studies already received extra specialist attention and were more frequently hospitalized.

As for the COPD GOLD I patients, overall survival did not differ from the general population. These data are in contrast to population cohort studies. ${ }^{2,4}$ Therefore, the data were studied in further detail and sub analysis did show an effect upon survival after exclusion of non-survivors within five years, possibly reflecting progression of COPD severity in this particular group, where loss of lung function is known to be accelerated. ${ }^{17}$ Of interest, smoking status especially influenced survival here, whereas smoking status did not particularly influence other GOLD stage groups. This dominant effect of smoking status upon survival in early COPD stage groups, but not in late groups, was also noted by others. ${ }^{18}$

Overall, the strongest predictors of mortality were age, FEV 1 , TLCO, Charlson comorbidity index, chronic respiratory insufficiency, and clinical exacerbation frequency (defined by mean number of admissions in first five years following inclusion). A weaker relationship was found for male gender, previous admission $<1$ year and ICU admission and no significant relationship with active smoking. In general, these results are supported by others. ${ }^{19-23}$ One problem in the field is that studies are often difficult to compare since most studies implemented a different set of variables. ${ }^{21}$ Some researchers have aimed to develop a prognostic scoring model to predict mortality after clinical exacerbation ${ }^{24}$, yet the lack of consensus about the variable set is clearly limiting these efforts. In addition, from time to time new variables are introduced, including biomarkers, physical activity measurements or presence of interstitial lung disease or sleep apnoea. ${ }^{19,25-28}$ Another approach using the standardized and generally applied hospital scoring system NEWS (National Early Warning Score) also appeared to be of limited value for COPD patients due to interference of the disease with the variables. $^{29}$

In our study, a low BMI $<18.5 \mathrm{~kg} / \mathrm{m}^{2}$ was a significant predictor of mortality only in bivariate analysis. The parameter was closely associated with a decreased TLCO and increased clinical exacerbation frequency, which obscured the influence of BMI in multivariable analysis in all COPD groups. This possibility of confounding factors may be taken into account when interpreting reports about the influence of a low BMI on mortality in COPD. ${ }^{30-32}$

One novel approach in our study was to differentiate prognostic factors among COPD GOLD classes. Despite the fact that many prognostic factors cannot be influenced such 
as gender or age, some factors may be special targets for treatment. As such, our findings may help to individualize treatment focus per COPD group. In all groups, apart from age, clinical exacerbation frequency was a significant risk factor. Therefore, every effort should be taken to prevent the need for hospitalization. For example, this may include an aggressive treatment approach by the general practitioner, methods to enhance cooperation between the general practitioner and specialist care and/or immediate delivery of care at the emergency department aiming to avoid hospital admission. As for COPD GOLD I and II, smoking cessation should be a primary goal to prevent any further loss of lung function. As for COPD GOLD III and IV, comorbidities should be actively looked for, weight and muscle mass should be monitored and correction of chronic hypercapnia by non-invasive home ventilation may be considered. ${ }^{33,34}$ Furthermore, the insights gained by our study may stimulate specific patient group targeting for other interventions not taken into account in the current study, like pulmonary rehabilitation or sleep apnoea treatment. ${ }^{25,26}$

The study of lung function parameters in our study demonstrated one interesting trend: whereas mortality within the earlier COPD groups was merely affected by a decreased diffusion capacity, in the later stages $\mathrm{FEV}_{1}$ was the predominant predictor of mortality. Hence, decreased diffusion capacity in COPD may be considered an early warning sign. Possibly, the association between decreased diffusion capacity and pulmonary hypertension may play a role here. ${ }^{35,36}$ However, cardiovascular mortality varied only little among the COPD groups. Another explanation may be a lack of physical reserves due to muscle wasting $^{37}$ since a decreased diffusion capacity correlated well with a low BMI.

In comparison to population cohort studies, our study design offers advantages in terms of a thorough, clinical characterization of our COPD patients. The risk of confounding asthma diagnoses was optimally reduced in our cohort by thorough chart review. Moreover, whereas the population cohort studies originated from 1971 to 1989, our cohort started in 1996, after publication of the first modern COPD guidelines. ${ }^{15}$ Another difference with population cohort studies is the relatively large proportion of COPD GOLD IV in our cohort. However, our design was limited by the small number of data available from the general population cohort (age, gender and date of death). As such, it was not possible to correct excess death rate data for other variables such as smoking status or BMI. In addition, some of these subjects may have been suffering from COPD themselves, since the estimated prevalence of COPD is estimated about $7.6 \%{ }^{38}$ In order to minimize this error, four control subjects were obtained for each COPD patient. 


\section{Conclusions}

In conclusion, long term mortality after clinical exacerbation of COPD appeared to be increased up to $82 \%$ in comparison the general population. Different factors contributed per COPD GOLD group. As such, every possible effort should be taken to reduce these factors in a personalized way. 


\section{References}

1. Lozano, R., et al., Global and regional mortality from 235 causes of death for 20 age groups in 1990 and 2010: a systematic analysis for the Global Burden of Disease Study 2010. Lancet. 380(9859): p. 2095128.

2. Mattila, T., et al., Association between all-cause and cause-specific mortality and the GOLD stages 1-4: A 30-year follow-up among Finnish adults. Respir Med. 109(8): p. 1012-8.

3. Mannino, D.M., et al., Lung function and mortality in the United States: data from the First National Health and Nutrition Examination Survey follow up study. Thorax, 2003. 58(5): p. 388-93.

4. Mannino, D.M., A. Sonia Buist, and W.M. Vollmer, Chronic obstructive pulmonary disease in the older adult: what defines abnormal lung function? Thorax, 2007. 62(3): p. 237-41.

5. Jensen, H.H., et al., Potential misclassification of causes of death from COPD. Eur Respir J, 2006. 28(4): p. 781-5.

6. Shavelle, R.M., et al., Life expectancy and years of life lost in chronic obstructive pulmonary disease: findings from the NHANES III Follow-up Study. Int J Chron Obstruct Pulmon Dis, 2009. 4: p. 137-48.

7. Dransfield, M.T., et al., Acute Exacerbations and Lung Function Loss in Smokers with and without Chronic Obstructive Pulmonary Disease. Am J Respir Crit Care Med. 195(3): p. 324-330.

8. Almagro, P., et al., Mortality after hospitalization for COPD. Chest, 2002. 121(5): p. 1441-8.

9. Soler-Cataluna, J.J., et al., Severe acute exacerbations and mortality in patients with chronic obstructive pulmonary disease. Thorax, 2005. 60(11): p. 925-31.

10. Miravitlles, M., et al., Costs of chronic bronchitis and COPD: a 1-year follow-up study. Chest, 2003. 123(3): p. 784-91.

11. Alfageme, I., et al., The effect of airflow limitation on the cause of death in patients with COPD. Chron Respir Dis. 7(3): p. 135-45.

12. Brusse-Keizer, M., et al., Comparing the 2007 and 2011 GOLD Classifications as Predictors of all-Cause Mortality and Morbidity in COPD. Copd. 14(1): p. 7-14.

13. Connors, A.F., Jr., et al., Outcomes following acute exacerbation of severe chronic obstructive lung disease. The SUPPORT investigators (Study to Understand Prognoses and Preferences for Outcomes and Risks of Treatments). Am J Respir Crit Care Med, 1996. 154(4 Pt 1): p. 959-67.

14. Slenter, R.H., et al., Predictors of 1-year mortality at hospital admission for acute exacerbations of chronic obstructive pulmonary disease. Respiration. 85(1): p. 15-26.

15. Standards for the diagnosis and care of patients with chronic obstructive pulmonary disease. American Thoracic Society. Am J Respir Crit Care Med, 1995. 152(5 Pt 2): p. S77-121.

16. Hillas, G., F. Perlikos, and N. Tzanakis, Acute exacerbation of COPD: is it the "stroke of the lungs"? Int J Chron Obstruct Pulmon Dis. 11: p. 1579-86.

17. Tantucci, C. and D. Modina, Lung function decline in COPD. Int J Chron Obstruct Pulmon Dis. 7: p. 95-9.

18. Ekberg-Aronsson, M., et al., Mortality in GOLD stages of COPD and its dependence on symptoms of chronic bronchitis. Respir Res, 2005. 6: p. 98.

19. Celli, B.R., Predictors of mortality in COPD. Respir Med. 104(6): p. 773-9.

20. Gudmundsson, G., et al., Long-term survival in patients hospitalized for chronic obstructive pulmonary disease: a prospective observational study in the Nordic countries. Int J Chron Obstruct Pulmon Dis. 7: p. 571-6.

21. Singanayagam, A., S. Schembri, and J.D. Chalmers, Predictors of mortality in hospitalized adults with acute exacerbation of chronic obstructive pulmonary disease. Ann Am Thorac Soc. 10(2): p. 81-9.

22. Ho, T.W., et al., In-hospital and one-year mortality and their predictors in patients hospitalized for firstever chronic obstructive pulmonary disease exacerbations: a nationwide population-based study. PLoS One. 9(12): p. e114866.

23. Flattet, Y., et al., Determining prognosis in acute exacerbation of COPD. Int J Chron Obstruct Pulmon Dis. 12: p. 467-475.

24. Vanasse, A., et al., Predicting One-year Mortality After a "First" Hospitalization for Chronic Obstructive Pulmonary Disease: An Eight-Variable Assessment Score Tool. Copd. 14(5): p. 490-497. 
25. Houchen-Wolloff, L., et al., Survival following pulmonary rehabilitation in patients with COPD: the effect of program completion and change in incremental shuttle walking test distance. Int J Chron Obstruct Pulmon Dis. 13: p. 37-44.

26. Marin, J.M., et al., Outcomes in patients with chronic obstructive pulmonary disease and obstructive sleep apnea: the overlap syndrome. Am J Respir Crit Care Med. 182(3): p. 325-31.

27. Waschki, B., et al., Physical activity is the strongest predictor of all-cause mortality in patients with COPD: a prospective cohort study. Chest. 140(2): p. 331-342.

28. Putman, R.K., et al., Association Between Interstitial Lung Abnormalities and All-Cause Mortality. Jama. 315(7): p. 672-81.

29. Hodgson, L.E., et al., A validation of the National Early Warning Score to predict outcome in patients with COPD exacerbation. Thorax. 72(1): p. 23-30.

30. Vestbo, J., et al., Body mass, fat-free body mass, and prognosis in patients with chronic obstructive pulmonary disease from a random population sample: findings from the Copenhagen City Heart Study. Am J Respir Crit Care Med, 2006. 173(1): p. 79-83.

31. Cao, C., et al., Body mass index and mortality in chronic obstructive pulmonary disease: a metaanalysis. PLoS One. 7(8): p. e43892.

32. Guo, Y., et al., Body mass index and mortality in chronic obstructive pulmonary disease: A doseresponse meta-analysis. Medicine (Baltimore). 95(28): p. e4225.

33. Struik, F.M., et al., Nocturnal non-invasive ventilation in COPD patients with prolonged hypercapnia after ventilatory support for acute respiratory failure: a randomised, controlled, parallel-group study. Thorax. 69(9): p. 826-34.

34. Murphy, P.B., et al., Effect of Home Noninvasive Ventilation With Oxygen Therapy vs Oxygen Therapy Alone on Hospital Readmission or Death After an Acute COPD Exacerbation: A Randomized Clinical Trial. Jama. 317(21): p. 2177-2186.

35. Nakahara, Y., et al., Exercise hypoxaemia as a predictor of pulmonary hypertension in COPD patients without severe resting hypoxaemia. Respirology. 22(1): p. 120-125.

36. Seeger, W., et al., Pulmonary hypertension in chronic lung diseases. J Am Coll Cardiol. 62(25 Suppl): p. D109-16.

37. van den Borst, B., H.R. Gosker, and A.M. Schols, Central fat and peripheral muscle: partners in crime in chronic obstructive pulmonary disease. Am J Respir Crit Care Med. 187(1): p. 8-13.

38. Halbert, R.J., et al., Global burden of COPD: systematic review and meta-analysis. Eur Respir J, 2006. 28(3): p. 523-32. 



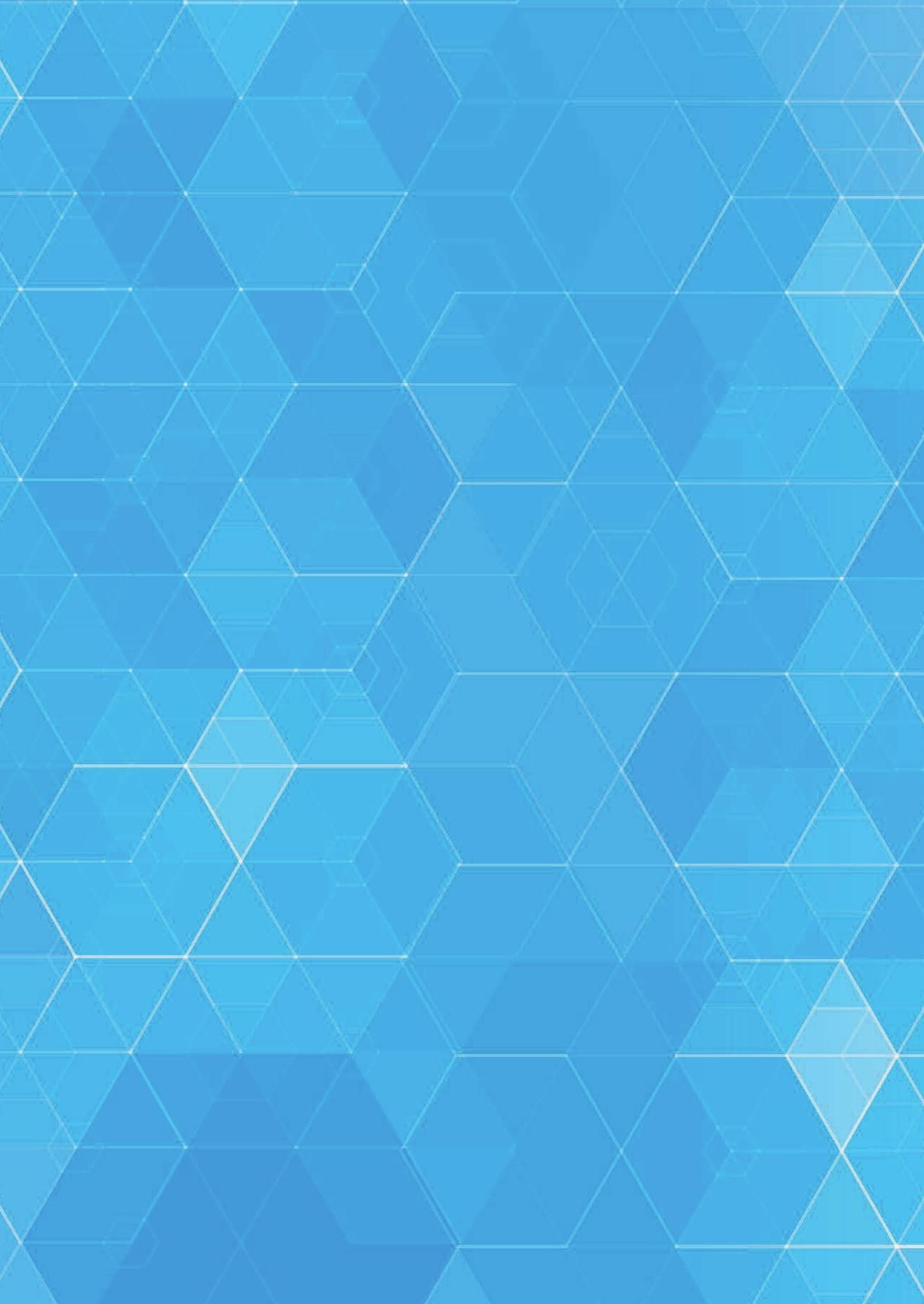




\section{Chapter 4}

Predictors of 1-year mortality at hospital admission for acute exacerbations of COPD

Roel H.J. Slenter ${ }^{*}$, Roy T.M. Sprooten*, Daniel Kotz, Geertjan Wesseling, Emiel F.M. Wouters, Gernot G.U. Rohde * These authors contributed equally to this work Published in Respiration 2013;85:15-26 


\section{Abstract}

\section{Background}

Acute exacerbations of chronic obstructive pulmonary disease (ECOPD) are related to high mortality, especially when hospitalized. Predictors for severe outcomes are still not sufficiently defined. The aim of the present study is (1) to assess the mortality rate and (2) to identify potential determinants of mortality in a cohort of patients hospitalized for ECOPD.

\section{Methods}

Retrospective, observational cohort study including all consecutive patients admitted between January 1, 2009 and April 1, 2010 for ECOPD. Potential predictors were assessed at initial presentation at the emergency room. The primary outcome was mortality during 1-year follow-up. Univariate and multivariate time-to-event analyses using Cox proportional hazard models were employed for statistical analysis.

\section{Results}

A total of 260 patients were enrolled in this study. Mean age was $70.5 \pm 10.8$ years, $50.0 \%$ were male and $63.4 \%$ had severe Chronic Obstructive Pulmonary Disease COPD. The in-hospital mortality rate was $5.8 \%$ and the 1-year mortality $27.7 \%$. Independent risk factors for mortality were age (Hazard Ratio $[H R]=1.04$; 95\% confidence interval $[\mathrm{Cl}]=1.01-1.07)$, male sex $(\mathrm{HR}=2.00 ; 95 \% \mathrm{Cl}=1.15-3.48)$, prior hospitalization for $\mathrm{ECOPD}$ in the last 2 years ( $\mathrm{HR}=2.56 ; 95 \% \mathrm{Cl}=1.52-4.30)$, prior recorded congestive heart failure ( $\mathrm{HR}=1.75 ; 95 \% \mathrm{Cl}=1.03-2.97), \mathrm{PaCO}_{2} \geq 6.0 \mathrm{kPa}(\mathrm{HR}=2.90 ; 95 \% \mathrm{Cl}=1.65-5.09)$ and urea $\geq 8.0 \mathrm{mmol} / \mathrm{l}(\mathrm{HR}=2.38 ; 95 \% \mathrm{Cl}=1.42-3.99)$ at admission.

\section{Conclusions}

Age, male sex, prior hospitalization for ECOPD in the last 2 years, prior recorded congestive heart failure, hypercapnia and elevated levels of urea at hospital admission are independent predictors of mortality within the first year after admission. 


\section{Introduction}

Chronic obstructive pulmonary disease (COPD) is one of the leading causes of death and disability worldwide that still has a rising prevalence and mortality rate. ${ }^{1,2}$ The natural course of COPD is characterized by a progressive decline in pulmonary function and recurrent exacerbations. Exacerbations are major events in the course of the disease, especially when hospitalization is required, and represent an important component of the socioeconomic burden related to COPD. ${ }^{3}$ An exacerbation of COPD is defined as an event in the natural course of the disease characterized by a change in the patient's baseline dyspnoea, cough, and/or sputum that is beyond normal day-today variations, is acute in onset, and may warrant a change in regular medication in a patient with underlying COPD. ${ }^{4}$ Mortality related to acute exacerbations of COPD (ECOPD) is particularly high during hospitalization, and the months thereafter. ${ }^{5}$ The inhospital mortality and also 1-year mortality vary markedly between studies depending on the mode of recruitment and setting, and range from $2.5-30 \%$ to $22-43 \%$ in relation to severity of disease. - $^{6}$

Despite being the only major disease showing increasing prevalence and mortality, factors that determine the short- and long-term outcomes of patients with COPD are not yet precisely understood. Several studies have identified predictive factors independently associated with an increased mortality risk due to COPD in general, and acute exacerbations in particular. Predictors thought to be related to mortality include age, body mass index (BMI), Forced Expiratory Volume in one second $\left(\mathrm{FEV}_{1}\right), \mathrm{pH}, \mathrm{PaCO}_{2}$, $\mathrm{PaO}_{2}$, blood chemistry, previous hospitalizations, specific treatments, cardiac factors and other comorbidities. ${ }^{6,7,10-18}$ However, independent prognostic factors are quite variable between studies, probably because of differences in patient populations, settings, duration of follow-up, collected variables and statistical methods used. In addition, most of these studies did not yet take non-invasive ventilation into account.

The present study was designed to assess the mortality rate and potential determinants of mortality in a cohort of patients admitted to Maastricht University Hospital for an ECOPD. Factors related to increased mortality rate were analysed in order to identify independent predictors for use in the hospital setting. 


\section{Methods}

\section{Study design and participants}

A retrospective, observational cohort study was performed including all consecutive patients who had been admitted to any of the pulmonary wards of Maastricht University Hospital for ECOPD. This university hospital has an important function as a regional hospital besides its academic function, and the patient population is therefore representative of a general hospital. A stepwise retrospective selection process was used for identification of patients. Patients were initially identified on the basis of ICD-9 codes 496 (COPD) and 786.0-9 (acute respiratory symptoms). Patients already hospitalized before January 1, 2009 were excluded. Discharge letters were screened and patients without a diagnosis of COPD were excluded. Further screening excluded patients without a final diagnosis of ECOPD, patients without administration of systemic glucocorticoids or patients initially admitted to another hospital and later referred to our hospital. Then spirometry data were analysed and patients with an ratio Forced Expiratory Volume in one second ( $\left.\mathrm{FEV}_{1}\right)$ /Vital Capacity (VC) (FEV $1 / F V$ ) above $70 \%$ were excluded (Figure 4.1).

Patients were enrolled between January 1, 2009 and April 1, 2010 according to the following inclusion criteria: established diagnosis of COPD as identified from the patient's pulmonary function test results $\left(\mathrm{FEV}_{1} / \mathrm{FVC}<70 \%\right.$ and $\mathrm{FEV}_{1}$ reversibility $\left.<11 \%\right)$ or, if unavailable, based on the patient's clinical history with compatible physical examination findings in accordance with the GOLD guidelines ${ }^{19}$; symptoms indicating a severe acute exacerbation of COPD requiring hospital admission; and treatment with systemic glucocorticoids. An exacerbation was defined by the presence of a sustained increase in at least two of the three following symptoms: dyspnoea; cough; and sputum purulence, requiring modification of regular treatment and necessitating admission to the hospital. ${ }^{20}$ The clinical setting consists of a regular pulmonary ward with integrated intermediate care available to all acute patients. Intensive care units are located on the same floor of the building. Non-invasive and also invasive ventilation was accessible immediately to all patients if needed.

Each patient was included only once in the study and received medical treatment at the discretion of the attending physician. Exclusion criteria included follow-up at a different hospital than Maastricht University Hospital, other primary diagnoses than ECOPD at hospital discharge, or objection against the use of patient data for research goals. The 
study protocol was reviewed and approved by the Hospital Research and Medical Ethics Committee.

Figure 4.1 Flowchart cohort selection.

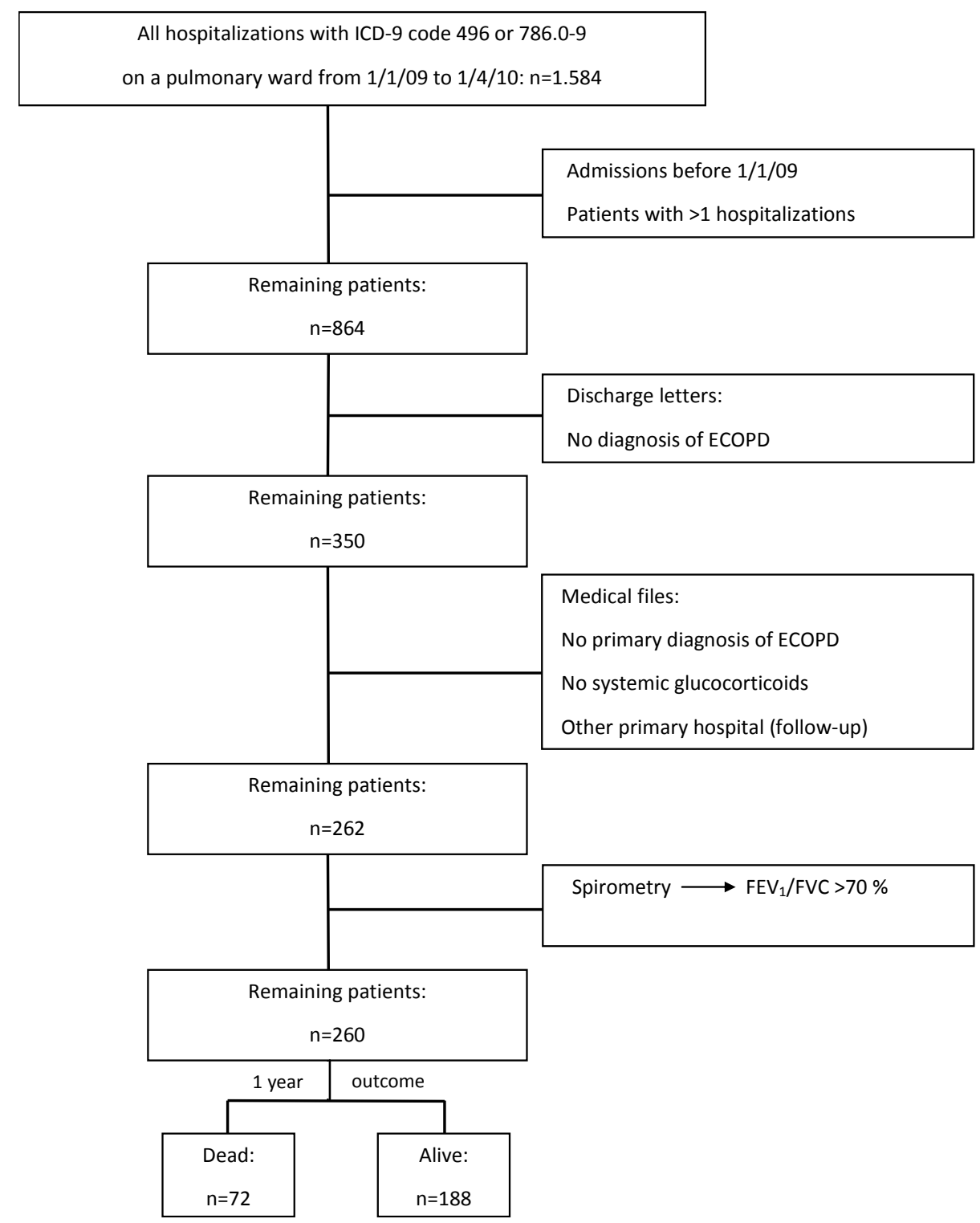

ICD-9 code 496: Chronic airway obstruction, not elsewhere classified (Chronic: nonspecific lung disease, obstructive lung disease, obstructive pulmonary disease [COPD] NOS). ICD-9 code 786-09: Symptoms involving respiratory system and other chest symptoms (Respiratory: distress, insufficiency). 


\section{Data collection}

Data were collected retrospectively from discharge letters, medical files and electronic patient records. If any data regarding pulmonary function testing or survival status was missing, we contacted the general practitioner or municipal personal records database.

\section{Measurements}

The following measurements were assessed during hospital stay: arterial blood gas levels, blood chemistry, need for intubation or non-invasive ventilation and active comorbidity. The decision to start and stop ventilation and the orders of supplementary arterial blood gas or blood chemistry reports were made on clinical grounds.

\section{Outcomes and study end points}

In-hospital mortality and length of hospital stay were determined for each patient. Patients were followed up for 1 year after hospital admission by review of clinical notes and electronic in-patient and out-patient records. Information on the following parameters were collected: survival status, survival time, cause of death, need of hospital readmission, number of hospital readmissions, need of hospital readmission for acute exacerbation of COPD and time to (first) readmission for acute exacerbation of COPD. The primary outcome was 1-year all-cause mortality, determined in the following ways. In case of an in-hospital death the date was recorded and verified by hospital records. The date of death of patients who died after hospital discharge was verified from hospital records, general practitioners records or the death records of the patients' residence. The survival status and cause of death of all patients was confirmed in this way. Unfortunately, mortality registration in the Netherlands does not allow access to actual records to determine the cause of death, which is why the cause of death could not be clarified in 23 of the deceased patients (31.9\%).

\section{Statistical analysis}

All analyses were performed using SPSS for Windows (version 18). Univariate analyses were performed using independent T-tests, $\mathrm{X}^{2}$ tests or Fisher's exact tests, as appropriate. Continuous variables that were not normally distributed were categorized and divided into clinically relevant groups prior to the analysis. A multiple Cox model for proportional hazards was used for our primary analysis of the independent effects of each variable on time to death. Potentially relevant clinical predictors, according to the univariate analyses $(p<0.05)$, were introduced into the starting model and then eliminated manually backwards step-by-step, dependent on the largest $p$-value. 
Patients with missing covariate data of any of the introduced predictors were excluded at starting point and remained so during the following process. All items showing statistical significance at $p<0.05$ were retained in the final prediction model.

\section{Results}

\section{Patient characteristics}

Between January 1, 2009, and April 1, 2010, 260 patients were admitted to any of the pulmonary wards of Maastricht University Hospital with a primary diagnosis of ECOPD and treated with systemic glucocorticoids. Patient selection and 1-year outcome are displayed in Figure 4.1 and the most important demographic characteristics are listed in Table 4.1. Mean age was $70.5 \pm 10.8$ years, and 130 patients $(50.0 \%)$ were male. The majority of the patients was retired (96.4\%), lived at home $(84.5 \%)$ with a partner or extra care (52.2\%) and had severe COPD defined as GOLD stage III-IV (63.4\%). One hundred thirty patients (50.0\%) had been previously admitted for a COPD exacerbation, $86(33.1 \%)$ had been hospitalized for this cause within the last 2 years and 23 (8.8\%) had received treatment with non-invasive positive pressure ventilation during this period. Of the study population, 242 (93.4\%) patients already had home-treatment for ECOPD, mostly intensified inhalation medication, and 69 (26.7\%) were on long-term oxygen therapy.

Clinically relevant comorbidity was present in more than half of all patients prior to hospital admission. Mainly cardiac and respiratory diseases were highly prevalent, in $65 \%$ and $60 \%$ of the patients, respectively. Also, $26.2 \%$ of patients had cancer and $30 \%$ peripheral vascular disease. Furthermore, psychiatric comorbidity had been recorded frequently, mainly composed of conditions like depression, anxiety and addiction. 
Table 4.1 Demographic and prior health-care utilization characteristics of patients hospitalized for acute exacerbation of COPD, stratified by 1-year outcome.

\begin{tabular}{|c|c|c|c|c|}
\hline Characteristics & All $(n=260)$ & Deceased $(n=72)$ & Alive $(n=188)$ & p-value \\
\hline \multicolumn{5}{|l|}{ Sex } \\
\hline Male & $130(50.0)$ & $45(62.5)$ & $85(45.2)$ & $<0.05^{\#}$ \\
\hline Female & $130(50.0)$ & $27(37.5)$ & $103(54.8)$ & \\
\hline Age, years & $70.5 \pm 10.8$ & $73.7 \pm 10.6$ & $69.3 \pm 10.7$ & $<0.05^{\#}$ \\
\hline \multicolumn{5}{|l|}{ Social status $(n=252 / 68 / 184)^{*}$} \\
\hline Retired $(n=251 / 68 / 183)^{*}$ & $242(96.4)$ & $67(98.5)$ & $175(95.6)$ & NS \\
\hline Living at own home & $213(84.5)$ & $54(79.4)$ & $159(86.4)$ & NS \\
\hline Living alone $(n=251 / 68 / 183)^{*}$ & $120(47.8)$ & $32(47.1)$ & $88(48.1)$ & NS \\
\hline \multicolumn{5}{|l|}{ GOLD stage $(n=246 / 65 / 181)^{*}$} \\
\hline 1 & $15(6.1)$ & $2(3.1)$ & $13(7.2)$ & $<0.10$ \\
\hline II & $75(30.5)$ & $15(23.1)$ & $60(33.1)$ & \\
\hline III & $77(31.3)$ & $19(29.2)$ & $58(32.1)$ & \\
\hline IV & $79(32.1)$ & $29(44.6)$ & $50(27.6)$ & \\
\hline $\mathrm{FEV}_{1}, \mathrm{~L}(n=245 / 64 / 181)^{*}$ & $1.05 \pm 0.42$ & $0.92 \pm 0.34$ & $1.09 \pm 0.44$ & $<0.05$ \\
\hline $\mathrm{FEV}_{1}, \%$ predicted $(n=246 / 65 / 181)^{*}$ & $45.0 \pm 18.0$ & $39.5 \pm 17.4$ & $47.0 \pm 17.8$ & $<0.05^{\#}$ \\
\hline FVC, $\%$ predicted $(n=246 / 65 / 181)^{*}$ & $84.5 \pm 22.7$ & $77.4 \pm 23.0$ & $87.1 \pm 22.2$ & $<0.05$ \\
\hline $\mathrm{FEV}_{1} / \mathrm{FVC}, \%(n=246 / 65 / 181)^{*}$ & $41.0 \pm 0.8$ & $39.2 \pm 11.6$ & $41.7 \pm 12.0$ & NS \\
\hline PEF, \% predicted $(n=245 / 64 / 181)^{*}$ & $52.9 \pm 19.2$ & $47.0 \pm 17.3$ & $55.0 \pm 19.5$ & $<0.05$ \\
\hline RV/TLC, \% (n=226/59/167)* & $60.0 \pm 11.0$ & $63.8 \pm 10.7$ & $58.7 \pm 10.7$ & $<0.05$ \\
\hline TLCO, \% predicted ( $n=213 / 55 / 158)^{*}$ & $47.4 \pm 19.3$ & $42.9 \pm 20.6$ & $49.0 \pm 18.6$ & $<0.05$ \\
\hline TLCO/VA, $\%$ predicted $(n=212 / 55 / 157) *$ & $58.8 \pm 23.8$ & $54.0 \pm 23.8$ & $60.4 \pm 23.7$ & $<0.10$ \\
\hline \multicolumn{5}{|l|}{ Hospital admissions for COPD } \\
\hline \multicolumn{5}{|l|}{ exacerbation in the last 2 year, No. } \\
\hline 0 & $174(66.9)$ & $39(54.2)$ & $135(71.8)$ & $<0.05$ \\
\hline 1 & $45(17.4)$ & $14(19.4)$ & $31(16.5)$ & \\
\hline $2-3$ & $30(11.5)$ & $12(16.7)$ & $18(9.6)$ & \\
\hline$\geq 4$ & $11(4.2)$ & $7(9.7)$ & $4(2.1)$ & \\
\hline \multicolumn{5}{|l|}{ Hospital admission for COPD } \\
\hline exacerbation in the last 2 year & $86(33.1)$ & $33(45.8)$ & $53(28.2)$ & $<0.05^{\#}$ \\
\hline Treated with antibiotics & $72(27.7)$ & $29(40.3)$ & 43 (22.9) & $<0.05$ \\
\hline Treated with NIV & $23(8.8)$ & $11(15.3)$ & $12(6.4)$ & $<0.05$ \\
\hline Treated with MV & $2(0.8)$ & $0(0.0)$ & $2(1.1)$ & NS \\
\hline Home treatment $(n=258 / 70 / 188)^{*}$ & $241(93.4)$ & $173(92.0)$ & $68(97.1)$ & NS \\
\hline Long-acting $\aleph_{2}$-agonists & $209(81.0)$ & $61(87.1)$ & $148(78.7)$ & NS \\
\hline Short-acting $ß_{2}$-agonists & $133(51.6)$ & 30 (42.9) & $103(54.8)$ & $<0.10$ \\
\hline Short-acting anticholinergs & $83(32.2)$ & $30(42.9)$ & $53(28.2)$ & $<0.05$ \\
\hline Long-acting anticholinergs & $156(60.5)$ & $43(61.4)$ & $113(60.1)$ & NS \\
\hline Inhaled glucocorticoids & 201 (77.9) & $55(78.6)$ & $146(77.7)$ & NS \\
\hline Long-term oral glucocorticoids & $38(14.7)$ & $15(21.4)$ & $23(12.2)$ & $<0.10$ \\
\hline Theophylline & $23(8.9)$ & $7(10.0)$ & $16(8.5)$ & NS \\
\hline LTOT & $69(26.7)$ & $27(38.6)$ & $42(22.3)$ & $<0.05^{\#}$ \\
\hline NIV & $9(3.5)$ & $5(7.1)$ & $4(2.1)$ & $<0.10$ \\
\hline CPAP & $3(1.2)$ & $1(1.4)$ & $2(1.1)$ & NS \\
\hline Antibiotics $^{\S}$ & $96(37.2)$ & $24(34.3)$ & $72(38.3)$ & NS \\
\hline
\end{tabular}

Data are presented as $\mathrm{n}(\%)$ or mean \pm SD. NIV: non-invasive positive pressure ventilation; MV: mechanical ventilation; LTOT: long-term oxygen therapy; CPAP: continuous positive airway pressure; NS: not significant. * ( $n=a$ all/dead/alive). \# Included in multivariate analysis. ${ }^{\S}$ pre-existing antibiotic treatment for the acute event. 


\section{Clinical data}

Forty-three patients (16.7\%) had a BMI below $20 \mathrm{~kg} / \mathrm{m}^{2}, 111(43.7 \%)$ were current smokers, 99 (39.1\%) had a pulse oxygen saturation value below $90 \%$ and 117 (46.4\%) had oxygen demand defined as the need for constant oxygen administration (Table 4.2). Signs of peripheral oedema and neurological impairment were present in 83 (31.9\%) and 26 (10.0\%) patients, respectively. Blood gas measurements showed acidosis, hypercapnia and moderate hypoxemia in 84 (32.7\%), 138 (53.7\%) and 121 (47.1\%) patients, respectively. Mean haemoglobin was $8.6 \pm 1.1 \mathrm{mmol} / \mathrm{l}, 77$ (30.1\%) patients had an urea $\geq 8.0 \mathrm{mmol} / \mathrm{I}$ and 54 (20.9\%) had increased creatinine levels. X-ray reports revealed signs of pneumonia and tumour in 71 (27.3\%) and 7 (2.7\%) cases, respectively. Patients with pneumonia were explicitly not excluded as the intention of this study was to give a real-life picture of COPD management, and up to now there is no consensus as how to deal with patients clinically presenting as ECOPD and concurrent pulmonary infiltrates. Moreover, the presence of pulmonary infiltrates could present a risk factor for mortality.

\section{Mortality and readmissions during 1-year follow-up}

Median hospital stay was 8 (5-14) days, and the majority of patients $(62.7 \%)$ was discharged within 10 days. The in-hospital mortality rate was $5.8 \%$ and the 1-year mortality rate $27.7 \%$ because of 15 and 72 deaths, respectively (see also additional table 3e). Of the 15 in-hospital deaths, 13 (86.7\%) were caused by respiratory failure. Of the 245 discharged patients, 135 (55.1\%) were readmitted within one year, $91(37.1 \%)$ of which because of acute exacerbations. The distribution between GOLD stage unknown and I-IV of the 91 readmitted patients was 3.3\%, 3.3\%, 27.5\%, 28.6\% and $37.4 \%$. These 91 patients had a total of 168 ECOPD readmissions in the first year of follow-up leading to an average readmission rate of 1.85 per readmitted patient, ranging from 1 to 6 , and a median time to readmission of 47 (21-156) days, ranging from 1 to 336 days. The majority of these patients received antibiotics (74.7\%) and $32(35.2 \%)$ have been treated with NIV during at least one admission. 
Table 4.2 Characteristics at Emergency Room presentation, stratified by 1-year outcome.

\begin{tabular}{|c|c|c|c|c|}
\hline Characteristics & All $(n=260)$ & Deceased $(n=72)$ & Alive $(n=188)$ & p-value \\
\hline \multicolumn{5}{|l|}{$\mathrm{BMI}, \mathrm{kg} / \mathrm{m}^{2}(n=258 / 70 / 188)^{*}$} \\
\hline$<20$ & $43(16.7)$ & $19(27.1)$ & $24(12.8)$ & $<0.05^{\#}$ \\
\hline $20-25$ & $122(47.3)$ & $34(48.6)$ & $88(46.8)$ & \\
\hline$>25$ & $93(36.0)$ & $17(24.3)$ & $76(40.4)$ & \\
\hline \multicolumn{5}{|l|}{ Smoking $(n=254 / 69 / 185)^{*}$} \\
\hline Current smoker & $111(43.7)$ & $31(44.9)$ & $80(43.2)$ & NS \\
\hline Ex-smoker & $140(55.1)$ & $36(52.2)$ & $102(56.3)$ & \\
\hline Non smoker & $3(1.2)$ & $2(2.9)$ & $1(0.5)$ & \\
\hline Pack Years $(n=129 / 20 / 109)^{*}$ & $43.8 \pm 16.1$ & $54.9 \pm 19.0$ & $41.8 \pm 14.7$ & $<0.05$ \\
\hline Oxygen saturation $<90 \%(n=253 / 69 / 184)^{*}$ & $99(39.1)$ & $25(36.2)$ & $74(40.2)$ & NS \\
\hline Additional oxygen $(n=252 / 68 / 184)^{*}$ & $117(46.4)$ & $44(64.7)$ & $73(39.7)$ & $<0.05$ \\
\hline Impaired consciousness & $26(10.0)$ & $12(16.7)$ & $14(7.4)$ & $<0.05^{\#}$ \\
\hline Use of accessory respiratory muscles & $52(20.0)$ & $14(19.4)$ & $38(20.2)$ & NS \\
\hline Elevated CVP & $26(10.0)$ & $10(13.9)$ & $16(8.5)$ & NS \\
\hline Peripheral edema & $83(31.9)$ & $29(40.3)$ & $54(28.7)$ & $<0.10$ \\
\hline Wheezing & $196(75.4)$ & $51(70.8)$ & $145(77.1)$ & NS \\
\hline Crepitations & $79(30.4)$ & $23(31.9)$ & $56(29.8)$ & NS \\
\hline \multicolumn{5}{|l|}{ Arterial blood gas $(n=257 / 72 / 185)^{*}$} \\
\hline $\mathrm{pH} \leq 7.25$ & $21(8.2)$ & $11(15.3)$ & $10(5.4)$ & $<0.05^{\#}$ \\
\hline $\mathrm{pH} 7.25-7.35$ & $63(24.5)$ & $23(31.9)$ & $40(21.6)$ & \\
\hline $\mathrm{pH} \geq 7.35$ & $173(67.3)$ & $38(52.8)$ & $135(73.0)$ & \\
\hline $\mathrm{PaCO}_{2}, \mathrm{kPa} \geq 6.0$ & $138(53.7)$ & $50(69.4)$ & $88(47.6)$ & $<0.05^{\#}$ \\
\hline $\mathrm{PaO}_{2}, \mathrm{kPa}<7.5$ & $121(47.1)$ & $32(44.4)$ & $89(48.1)$ & NS \\
\hline $\mathrm{BE}, \mathrm{mmol} / \mathrm{I} \geq 2.5$ & $105(40.9)$ & $37(51.4)$ & $68(36.8)$ & $<0.05^{\#}$ \\
\hline $\mathrm{HCO}_{3}^{-}, \mathrm{mmol} / \mathrm{l} \geq 27.0$ & $85(33.1)$ & $32(44.4)$ & $53(28.6)$ & $<0.05$ \\
\hline $\mathrm{SaO}_{2}<90 \%$ & $131(51.0)$ & $38(52.8)$ & $93(50.3)$ & NS \\
\hline Hemoglobin, $\mathrm{mmol} / \mathrm{I}(n=258 / 72 / 186)^{*}$ & $8.6 \pm 1.1$ & $8.3 \pm 1.4$ & $8.8 \pm 0.9$ & $<0.05^{\#}$ \\
\hline WBC, $10^{9} / I>15.0(n=259 / 72 / 187)^{*}$ & $69(26.6)$ & $24(33.3)$ & $45(24.1)$ & NS \\
\hline Glucose, $\mathrm{mmol} / \mathrm{l}>11,0(n=246 / 69 / 177)^{*}$ & $36(14.6)$ & $13(18.8)$ & $23(13.0)$ & NS \\
\hline Sodium, $\mathrm{mmol} / \mathrm{l}<135(n=257 / 72 / 185)^{*}$ & $50(19.5)$ & $14(19.4)$ & $36(19.5)$ & NS \\
\hline Urea, $\mathrm{mmol} / \mathrm{l} \geq 8.0(n=256 / 70 / 186)^{*}$ & $77(30.1)$ & $32(45.7)$ & $45(24.2)$ & $<0.05^{\#}$ \\
\hline Creatinine, $\mu \mathrm{mol} / \mathrm{l}>115(n=258 / 72 / 186)^{*}$ & $54(20.9)$ & $23(31.9)$ & $31(16.7)$ & $<0.05$ \\
\hline CRP, $\mathrm{mg} / \mathrm{l}<50(n=257 / 72 / 185)^{*}$ & 141 (54.9) & $36(50.0)$ & $105(56.8)$ & $<0.05^{\#}$ \\
\hline $50-200$ & $80(31.1)$ & $31(43.1)$ & $49(26.5)$ & \\
\hline$>200$ & $36(14.0)$ & $5(6.9)$ & $31(16.7)$ & \\
\hline \multicolumn{5}{|l|}{ Chest $\mathrm{X}$-ray findings } \\
\hline Pulmonary edema & $26(10.0)$ & $12(16.7)$ & $14(7.4)$ & $<0.10$ \\
\hline Pneumonia & $71(27.3)$ & $23(31.9)$ & $48(25.5)$ & NS \\
\hline Tumour & $7(2.7)$ & $4(5.6)$ & $3(1.6)$ & $<0.10$ \\
\hline Heart failure & $34(13.1)$ & $10(13.9)$ & $24(12.8)$ & NS \\
\hline
\end{tabular}

Data are presented as No. (\%) or mean \pm SD. NS: not significant; BMI: body mass index; Pack years: 20 cigarettes per day for 1 year; CVP: central venous pressure; WBC: white blood cells; CRP: C-reactive protein. * $(\mathrm{n}=$ all/dead/alive) \# Included in multivariate analysis.

\section{Predictors of mortality}

Univariate analyses were performed on all available data to identify variables associated with increased 1-year mortality. These variables included male sex, 


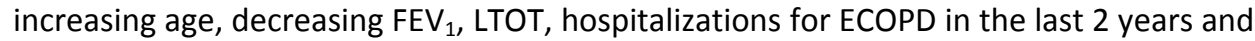
certain comorbidities (cardiac arrhythmia, congestive heart failure, pneumonia) recorded prior to the current admission (tables 1 and 1e). Further risk factors at hospital admission associated with lower survival rates included a BMI below $20 \mathrm{~kg} / \mathrm{m}^{2}$, abnormalities in arterial blood gas $\left(\mathrm{pH}, \mathrm{PaCO}_{2}\right.$, base excess $\left.(\mathrm{BE})\right)$ and blood values (hemoglobin, urea, C-reactive protein (CRP); Table 4.2).

Of the original 260 patients, 24 were excluded because of missing covariate data, leaving 236 patients for the primary analysis. Table 4.3 shows the independent predictors of 1-year mortality at hospital admission for ECOPD that remained in the final model: age (Hazard Ratio $[H R]=1.04 ; 95 \%$ confidence interval $[\mathrm{Cl}]=1.01-1.07$ ), male sex $(\mathrm{HR}=2.00 ; 95 \% \mathrm{Cl}=1.15-3.48)$, prior hospitalization for ECOPD in the last 2 years ( $\mathrm{HR}=2.56 ; 95 \% \mathrm{Cl}=1.52-4.30)$, prior recorded congestive heart failure ( $\mathrm{HR}=1.75 ; 95 \%$ $\mathrm{Cl}=1.03-2.97), \mathrm{PaCO}_{2} \geq 6.0 \mathrm{kPa}(\mathrm{HR}=2.90 ; 95 \% \mathrm{Cl}=1.65-5.09)$ and urea $\geq 8.0 \mathrm{mmol} / \mathrm{l}$ $(\mathrm{HR}=2.38 ; 95 \% \mathrm{Cl}=1.42-3.99)$ at admission.

Table 4.3 Predictors of 1-year mortality measured at hospital admission for ECOPD: multivariate analysis (Cox proportional hazards).

\begin{tabular}{lccc}
\hline Characteristics & HR & $\mathbf{9 5 \%} \mathbf{C l}$ & p-value \\
\hline Sex, male & 2.00 & $1.15-3.48$ & 0.014 \\
Age, years & 1.04 & $1.01-1.07$ & 0.016 \\
Hospital admission for COPD exacerbation in the last 2 year prior & 2.56 & $1.52-4.30$ & $<0.001$ \\
$\quad$ to current admission & & & \\
Congestive heart failure, documented prior to current admission & 1.75 & $1.03-2.97$ & 0.038 \\
$\mathrm{PaCO}_{2}, \mathrm{kPa} \geq 6.0$ & 2.90 & $1.65-5.09$ & $<0.001$ \\
Urea, $\mathrm{mmol} / \mathrm{I} \geq 8.0$ & 2.38 & $1.42-3.99$ & 0.001 \\
\hline
\end{tabular}

HR: hazard ratio; Cl: confidence interval. Total patients 236 (dead: 61, alive: 175).

\section{Characteristics of patients stratified by gender}

Male patients had significantly lower spirometric values (FEV 1 , FVC, FEV $1 / F V C$ ), more comorbidities (cardiac and respiratory) and higher serum CRP and creatinine levels (Table 4.4). None of these variables, however, appeared to be independently associated with an increased risk of death.

\section{Survival of patients stratified by gender}

Figure 4.2 shows the cumulative survival of patients stratified by gender. Male patients with confirmed COPD by spirometry showed a significantly decreased rate of survival compared to female patients $(p=0.004)$. 
Table 4.4 Selected characteristics of patients hospitalized for acute exacerbation of COPD stratified by gender.

\begin{tabular}{|c|c|c|c|c|}
\hline Characteristics & All $(n=260)$ & Female $(n=130)$ & Male $(n=130)$ & p-value \\
\hline Age, years & $70.5 \pm 10.8$ & $69.8 \pm 11.2$ & $71.2 \pm 10.5$ & NS \\
\hline \multicolumn{5}{|l|}{ Social status $(n=252 / 127 / 125)^{*}$} \\
\hline Retired $(n=251 / 127 / 124)^{*}$ & $242(96.4)$ & $124(97.6)$ & $118(95.2)$ & NS \\
\hline Living at own home & $213(84.5)$ & $108(85.0)$ & $105(84.0)$ & NS \\
\hline Living alone $(n=251 / 127 / 124)^{*}$ & $120(47.8)$ & $74(58.3)$ & $46(37.1)$ & $<0.05$ \\
\hline \multicolumn{5}{|l|}{ GOLD stage $(n=246 / 120 / 126)^{*}$} \\
\hline 1 & $15(6.1)$ & $13(10.8)$ & $2(1.6)$ & $<0.05$ \\
\hline II & $75(30.5)$ & $43(35.8)$ & $32(25.4)$ & \\
\hline III & $77(31.3)$ & $31(25.8)$ & $46(36.5)$ & \\
\hline IV & $79(32.1)$ & $33(27.5)$ & $46(36.5)$ & \\
\hline $\mathrm{FEV}_{1}, \mathrm{~L}(n=245 / 120 / 125)^{*}$ & $1.05 \pm 0.42$ & $0.94 \pm 0.36$ & $1.15 \pm 0.45$ & $<0.05$ \\
\hline $\mathrm{FEV}_{1}, \%$ predicted $(n=246 / 120 / 126)^{*}$ & $45.0 \pm 18.0$ & $49.6 \pm 19.4$ & $40.6 \pm 15.3$ & $<0.05$ \\
\hline FVC, $\%$ predicted $(n=246 / 120 / 126)^{*}$ & $84.5 \pm 22.7$ & $90.1 \pm 23.9$ & $79.2 \pm 20.3$ & $<0.05$ \\
\hline Tiffeneau, \% ( $n=246 / 120 / 126)^{*}$ & $41.0 \pm 0.8$ & $43.6 \pm 11.7$ & $38.6 \pm 11.6$ & $<0.05$ \\
\hline \multirow{2}{*}{\multicolumn{5}{|c|}{$\begin{array}{l}\text { Hospital admissions for COPD } \\
\text { exacerbation in the last } 2 \text { year, No. }\end{array}$}} \\
\hline & & & & \\
\hline 0 & $174(66.9)$ & $91(70.0)$ & $83(63.8)$ & $<0.05$ \\
\hline 1 & $45(17.4)$ & $26(20.0)$ & $19(14.6)$ & \\
\hline $2-3$ & $30(11.5)$ & $11(8.5)$ & $19(14.6)$ & \\
\hline$\geq 4$ & $11(4.2)$ & $2(1.5)$ & $9(6.9)$ & \\
\hline \multicolumn{5}{|l|}{ Hospital admission for COPD } \\
\hline exacerbation in the last 2 year & $86(33.1)$ & $39(30.0)$ & $47(36.2)$ & NS \\
\hline Treated with antibiotics & $72(27.7)$ & $30(23.1)$ & $42(32.2)$ & $<0.10$ \\
\hline Treated with NIV & $23(8.8)$ & $14(10.8)$ & $9(6.9)$ & NS \\
\hline Treated with MV & $2(0.8)$ & $1(0.8)$ & $1(0.8)$ & NS \\
\hline Comorbidities** & $169(65.0)$ & 87 (66.9) & $82(63.1)$ & NS \\
\hline Cardiac comorbidities & $58(22.3)$ & $23(17.7)$ & 35 (26.9) & $<0.10$ \\
\hline \multicolumn{5}{|l|}{ Congestive heart failure } \\
\hline Respiratory comorbidities & $156(60.0)$ & $69(53.1)$ & 87 (66.9) & $<0.05$ \\
\hline Pneumonia*** & $82(31.5)$ & $31(23.8)$ & $51(39.2)$ & $<0.05$ \\
\hline Pneumothorax & $15(5.8)$ & $2(1.5)$ & $13(10.0)$ & $<0.05$ \\
\hline Psychiatric comorbidities & $47(18.1)$ & $23(17.7)$ & $24(18.5)$ & NS \\
\hline Depression/anxiety & $27(10.4)$ & $18(13.8)$ & $9(6.9)$ & $<0.10$ \\
\hline Addiction & $21(8.1)$ & $4(3.1)$ & $17(13.1)$ & $<0.05$ \\
\hline Gastro-intestinal disease & $39(15.0)$ & $21(16.2)$ & $18(13.8)$ & NS \\
\hline Gastrointestinal bleeding & $11(4.2)$ & $10(7.7)$ & $1(0.8)$ & $<0.05$ \\
\hline \multicolumn{5}{|l|}{ Further characteristics } \\
\hline Peripheral oedema & 83 (31.9) & $34(26.2)$ & $49(37.7)$ & $<0.05$ \\
\hline Creatinine, $\mu \mathrm{mol} / \mathrm{l}>115(n=258 / 129 / 129)^{*}$ & $54(20.9)$ & $18(14.0)$ & $36(27.9)$ & $<0.05$ \\
\hline \multicolumn{5}{|l|}{$\mathrm{CRP}, \mathrm{mg} / \mathrm{l}$} \\
\hline$<50(n=257 / 129 / 128)^{*}$ & $141(54.9)$ & $79(61.2)$ & $62(48.4)$ & $<0.10$ \\
\hline $50-200$ & $80(31.1)$ & $37(28.7)$ & $43(33.6)$ & \\
\hline$>200$ & $36(14.0)$ & $13(10.1)$ & $23(18.0)$ & \\
\hline \multicolumn{5}{|l|}{ Chest X-ray findings } \\
\hline Pneumonia & $71(27.3)$ & $26(20.0)$ & $45(34.6)$ & $<0.05$ \\
\hline
\end{tabular}

Data are presented as $n(\%)$ or mean \pm SD. NPPV: non-invasive ventilation; MV: mechanical ventilation; NS: not significant. ${ }^{*}$ (n=all/female/male) ${ }^{* *}$ Comorbid conditions do not have to be active. ${ }^{* *}$ recorded by pulmonary physician. 


\section{Sensitivity analysis}

To assess the potential influence of patients who did not have a (recent) lung function test to confirm the diagnosis or stage of COPD, we created Cox proportional hazards models without $\mathrm{FEV}_{1}$ as potential predictor, thereby including patients with missing values for pulmonary function tests. These models were not substantially different from the primary models that included $\mathrm{FEV}_{1}$ for any of the significant variables.

Figure 4.2 Cumulative survival of patients with confirmed COPD by spirometry stratified by gender ( $n=246$, $\mathrm{p}=0.004)$.

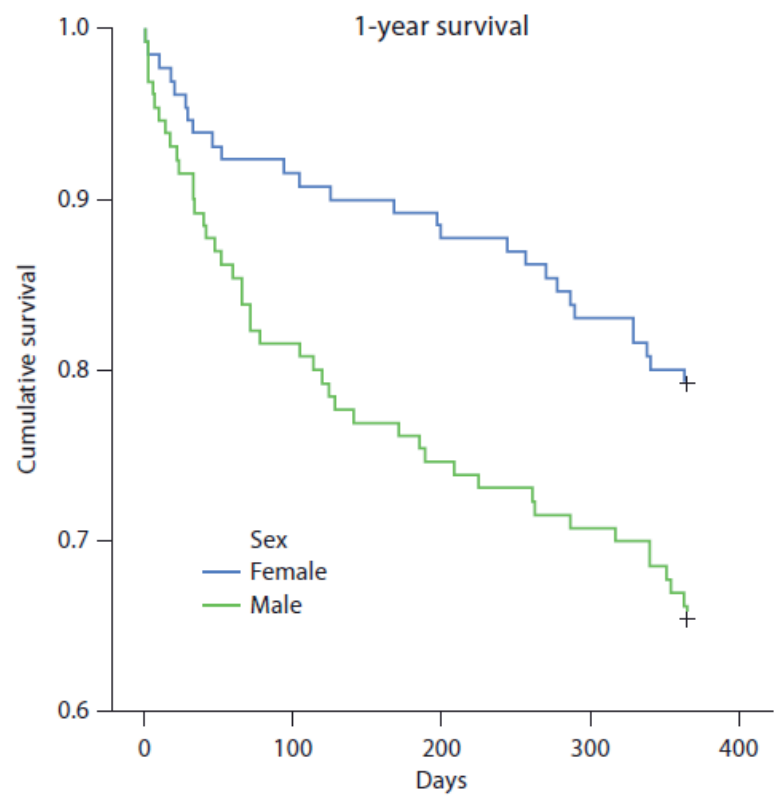

\section{Discussion}

The purpose of the present study was to determine predictors of mortality in patients hospitalized for acute exacerbations of COPD. We identified 6 simple, immediately accessible and strong predictors of 1-year mortality at hospital admission for acute exacerbation of COPD: age, male sex, prior hospitalization for ECOPD in the last 2 years, prior recorded congestive heart failure, hypercapnia and elevated urea at hospital admission. 
We observed an in-hospital mortality rate of $5.8 \%$ and a 1-year mortality rate of $27.7 \%$ which is similar to findings of other studies, demonstrating a high mortality in cohorts of unselected patients after hospitalization for ECOPD, both in-hospital and during 1year follow-up. ${ }^{6,7,21}$ It is important to acknowledge that mortality continues to rise, even after hospital discharge, which is only in part attributable to the natural course of the disease. The main cause of in-hospital death in our population was respiratory failure. A recent post-mortem analysis of major causes of early death in patients hospitalized with COPD exacerbation found that cardiac failure was the most frequent primary cause of death followed by pneumonia and pulmonary thromboembolism. Respiratory failure only ranked fourth as primary cause of death in this study. ${ }^{22}$ However, all patients died within 24 hours of admission to the hospital in this study whereas in our study almost all patients died much later after admission (142 \pm 125 days). Therefore these patients may represent a different group of high-risk patients compared to our patients.

Another important aspect is that during follow-up, the number of hospital readmissions was high, with $55.1 \%$ of patients having one or more hospital readmissions, and $37.1 \%$ of the population having been readmitted because of an acute exacerbation. This is similar to other reports and highlights the importance of ECOPD requiring hospital admission for the course of the disease. ${ }^{21}$

Significant findings in this study include the fact that increasing age, male sex, prior hospitalization for ECOPD, prior recorded congestive heart failure, $\mathrm{PaCO}_{2} \geq 6.0 \mathrm{kPa}$ and urea $\geq 8.0 \mathrm{mmol} / \mathrm{I}$ at hospital admission seem to be independent predictors associated with increased mortality. Aside from male sex, most of these factors have been well established in previous work, and some have also been applied in prediction models. $7,17,21,23$

Male sex is an independent predictor of death in our study. The effect of gender on mortality in patients with COPD remains controversial. To our knowledge, only a few studies have previously shown an increased risk of death in men with COPD, and only found a small influence. ${ }^{8,21,24}$ A possible explanation why most reports did not find a gender effect could be the fact that the majority of these studies included only a few or no female patients at all, therefore lacking the possibility of detecting an actual difference. Due to the gender distribution in our patient population, this study appears to be ideal to address this issue and we are therefore the first to report such a prominent association of male sex with an increased risk of death. In comparison with female patients, men had significant lower spirometric values (FEV $\left.1, F V C, F E V_{1} / F V C\right)$, 
more comorbidity (cardiac and respiratory), higher serum CRP and creatinine levels. None of these variables however, appeared to be independently associated with an increased risk of death. However, these findings do indicate a poorer health status of male COPD patients admitted to hospital with an ECOPD.

Our findings and those of other studies suggest that a history of hospitalization for ECOPD identifies a subgroup of COPD patients with a poor prognosis. ${ }^{6,14,15,17,25}$ We observed a higher rate of mortality among patients who had been admitted in the 2 years prior to the study period. Furthermore, the frequency of admissions and treatment with antibiotics or NIV during these admissions are also associated with increased mortality. Our findings support previous reports about the influence of hospital admission on mortality in ECOPD. ${ }^{26}$

The presence of congestive heart failure was an independent predictor of 1-year mortality in our study. The effect of comorbidity on mortality in general is complex. Instead of compressing all comorbidity into an index like the Charlson index, we explored the impact of specific comorbid conditions separately. ${ }^{27}$ Selected diseases like heart failure, cardiac ischemia and pneumonia have been previously shown to influence the prognosis in severe COPD patients. ${ }^{13,15,28-30}$ Congestive heart failure as well as COPD has to be considered as advanced chronic organ failure. Clinically, these entities are difficult to differentiate, as symptoms are very similar. Moreover both diseases often coexist and patients suffer from multiple symptoms, which are often under treated. ${ }^{31,32}$ Acute left heart dysfunction has been shown to be present in $25-30 \%$ of patients with ECOPD. ${ }^{33,34}$ Furthermore, also other studies have shown that congestive heart failure is an independent risk factor for survival in patients with COPD who had acute exacerbations requiring hospitalization. ${ }^{15}$

We found an increased $\mathrm{PaCO}_{2}$ to be a significant risk factor for mortality in our population, similar to findings of other studies. ${ }^{7,14,35,36}$ Hypercapnia is a result of alveolar hypoventilation. In COPD it results mainly from severe airflow limitation and hyperinflation, which increase during acute exacerbations. The respiratory muscle load, which is already high during stable state, may increase and a respiratory muscle fatigue may develop. Hypercapnia and respiratory acidosis can further decrease respiratory muscle function due to the deleterious effects of acidosis on mitochondrial metabolism and decreased energy production. A vicious circle may develop leading to increased morbidity and mortality. 
An interesting finding of this study is that increased urea levels were predictive of mortality. This is in analogy to the situation in community acquired pneumonia (CAP). ${ }^{37}$ In COPD clinically stable patients with hypercapnia show impaired secretion of sodium and water and correlations have been found between the degree of hypercapnia and the impairment in sodium excretion. ${ }^{38}$ Moreover hypoxemia decreases urinary sodium output probably related to a decline in glomerular filtration rate. Correction of hypoxemia has been shown to result in increased natriuresis. ${ }^{39}$ Moreover renal blood flow is severely decreased during ECOPD, extensive oedema may develop and hypercapnia even further diminishes renal blood flow. ${ }^{40}$ All these mechanisms may help to understand why elevated urea is predictive of mortality in ECOPD and highlights the complex interactions of different pathophysiological factors. Interestingly most of the relevant pathophysiological factors also play an important role as predictors of mortality. Furthermore it has been shown that sufficient metabolic compensation of respiratory acidosis and adequate renal function significantly decrease mortality. ${ }^{41}$

Although all our patients presented with the clinical picture of ECOPD and clearly fulfilled the proposed and generally accepted criteria for ECOPD, 71 of them showed infiltrates on chest $\mathrm{X}$-ray and were retrospectively also diagnosed with pneumonia. This represents a common clinical situation and up to now there is no consensus on how to categorize these patients. Almost all of them (93\%) were treated with antibiotics and thus received efficient therapy. If we excluded these 71 patients from the multivariate analysis the variables age, gender, prior hospitalization for ECOPD and hypercapnia remained significantly related to mortality. Urea was the last variable to be excluded from analysis $(p=0.075)$ just preceded by prior recorded congestive heart failure. This finding allows 2 different conclusions. First, the identification of elevated urea as a predictor of mortality was driven by ECOPD patients with pulmonary infiltrates. This would imply that detection of elevated urea in ECOPD patients identifies a subgroup of patients with probable pneumonia at increased risk of death. Second, it seems also plausible that elevation in urea is a marker of specific pathophysiological changes (see above) associated with increased mortality permitting progression of airways infection to parenchymal infection. Similar reasoning may explain the influence of prior recorded congestive heart failure on mortality, but further research is clearly warranted to clarify these important aspects.

Changes in lung function play an important role in the pathophysiology of COPD and almost all spirometric measurements were predictive of mortality in univariate analysis but not in multivariate analysis. In particular, no predictive value was suggested when $\mathrm{FEV}_{1}$ was assessed in multivariate analysis, despite being the most widely used 
parameter of lung function impairment and its role in classification of the disease into GOLD stages. ${ }^{19}$ On the one hand this might be explained by the low variability of FEV 1 values in our patients, making differentiating between survivors and non-survivors difficult. Although almost all patients (94.6\%) in our study have had a lung function measurement to confirm the diagnosis of COPD, a considerable proportion did not have a pulmonary function test within 3 years of index hospitalization. The accuracy and validity of these measurements are therefore at least questionable, especially for application in a prediction model to be used at hospital admission. However this represents a frequently encountered situation of daily clinical practice, as many COPD patients do not present with recent lung function measurements. On the other hand this result is not surprising, as $\mathrm{FEV}_{1}$ as well as other lung function parameters have been shown to be poor predictors of mortality in stable disease. ${ }^{42}$ Moreover FEV $_{1}$ measured at exacerbation might well be an important predictor but cannot be measured during acute exacerbation in most patients.

Similar to other studies we found an influence of BMI on survival, showing that low body weight is a predictor of long-term mortality in hospitalized patients with ECOPD. ${ }^{15,35,43,44}$ Our results support the concept that wasting represents an important component of systemic manifestations of COPD related to morbidity and mortality.

Although current smoking status did not have any influence on mortality, the amount of pack years smoked did clearly have predictive value. Unfortunately, this parameter was not recorded systematically and we could not use this variable for the multivariate analysis. However, these results support the notion that smoking has a negative effect on different features of COPD and therefore should be regarded as an important risk factor for death.

Our study has some limitations. First, it is a retrospective, single-centre cohort study and data might not be representative for other centers. Because we used administrative data, we were limited by the accuracy with which diagnoses were routinely coded, so we might have missed some patients. The sample size of 260 patients allows for reliable analyses but might have been too low to detect other potentially important predictors. Furthermore the sample size might be too small to extrapolate our findings to other settings because it was too low for cross-validating the prediction model. Further research is needed to externally validate the model in a different patient population. 
Second, assessments of variables were not standardized due to the retrospective design of the study. Results of spirometry were not obtained in a systematic way making the accuracy and validity of these values at least questionable. Nevertheless, we are convinced that data presented here are accurate because of the meticulous criteria used and validation of the data as outlined above. Finally, the high prevalence of (active) comorbidity has to be taken into account when interpreting the results, although it reflects to what happens in daily practice.

We believe, however, that these limitations did not significantly influence the main findings of the present study. Moreover, the data obtained represent clinical reality and may therefore be very relevant to clinical practice. Our work identified several variables that may be useful predictors of mortality at hospital admission for acute exacerbation of COPD. Strengths of our study include the ideal gender distribution, the amount of collected data and the fact that the diagnosis of COPD in this study relied on original doctor's records and was confirmed by lung function in almost all patients. All patients finally included into the study definitively had a diagnosis of ECOPD.

\section{Conclusions}

The present study provides clear prognostic factors for use in patients with an acute exacerbation of chronic obstructive pulmonary disease requiring hospitalization presenting to the emergency room. Age, male sex, prior hospitalization for ECOPD in the last 2 years, prior recorded congestive heart failure, $\mathrm{PaCO}_{2} \geq 6.0 \mathrm{kPa}$ and urea $\geq 8.0$ $\mathrm{mmol} / \mathrm{l}$ at hospital admission represent powerful predictors of the risk of death within the first year after admission. These predictors are easily registered at the ER rendering an application in a general clinical setting possible. 


\section{References}

1. Hurd, S., The impact of COPD on lung health worldwide: epidemiology and incidence. Chest, 2000. 117(2 Suppl): p. 1S-4S.

2. Mannino, D.M., et al., The natural history of chronic obstructive pulmonary disease. Eur Respir J, 2006. 27(3): p. 627-43.

3. Rutten-van Molken, M.P., et al., Current and future medical costs of asthma and chronic obstructive pulmonary disease in The Netherlands. Respir Med, 1999. 93(11): p. 779-87.

4. Global Strategy for the Diagnosis, Management and Prevention of COPD, Global Initiative for Chronic Obstructive Lung Disease (GOLD) 2010. 2010 25.10.2011]; Available from: http://www.goldcopd.org/.

5. Roberts, C.M., et al., Clinical audit indicators of outcome following admission to hospital with acute exacerbation of chronic obstructive pulmonary disease. Thorax, 2002. 57(2): p. 137-141.

6. Almagro, P., et al., Mortality after hospitalization for COPD. Chest, 2002. 121(5): p. 1441-8.

7. Groenewegen, K.H., A.M. Schols, and E.F. Wouters, Mortality and mortality-related factors after hospitalization for acute exacerbation of COPD. Chest, 2003. 124(2): p. 459-67.

8. Patil, S.P., et al., In-hospital mortality following acute exacerbations of chronic obstructive pulmonary disease. Arch Intern Med, 2003. 163(10): p. 1180-6.

9. Wang, Q. and J. Bourbeau, Outcomes and health-related quality of life following hospitalization for an acute exacerbation of COPD. Respirology, 2005. 10(3): p. 334-40.

10. Tan, W.C., Factors associated with outcomes of acute exacerbations of chronic obstructive pulmonary disease. Copd, 2004. 1(2): p. 225-47.

11. Yang, S., et al., Acute exacerbation of COPD requiring admission to the intensive care unit. Respirology, 2004. 9(4): p. 543-9.

12. Gunen, H., et al., Factors affecting survival of hospitalised patients with COPD. Eur Respir J, 2005. 26(2): p. 234-41.

13. Fuso, L., et al., Predicting mortality of patients hospitalized for acutely exacerbated chronic obstructive pulmonary disease. Am J Med, 1995. 98(3): p. 272-7.

14. Seneff, M.G., et al., Hospital and 1-year survival of patients admitted to intensive care units with acute exacerbation of chronic obstructive pulmonary disease. JAMA, 1995. 274(23): p. 1852-1857.

15. Connors, A.F., Jr., et al., Outcomes following acute exacerbation of severe chronic obstructive lung disease. The SUPPORT investigators (Study to Understand Prognoses and Preferences for Outcomes and Risks of Treatments). Am J Respir Crit Care Med, 1996. 154(4 Pt 1): p. 959-67.

16. Antonelli Incalzi, R., et al., Co-morbidity contributes to predict mortality of patients with chronic obstructive pulmonary disease. Eur Respir J, 1997. 10(12): p. 2794-800.

17. Soler-Cataluna, J.J., et al., Severe acute exacerbations and mortality in patients with chronic obstructive pulmonary disease. Thorax, 2005. 60(11): p. 925-31.

18. Matkovic, Z., et al., Predictors of Adverse Outcome in Patients Hospitalised for Exacerbation of Chronic Obstructive Pulmonary Disease. Respiration, 2012.

19. Global initiative for chronic obstructive lung disease. Global Strategy for the Diagnosis, Management and Prevention of COPD, Global Initiative for Chronic Obstructive Lung Disease (Revised 2011). 2011 03.05.2012]; Available from: www.goldcopd.org.

20. Anthonisen, N.R., et al., Antibiotic therapy in exacerbations of chronic obstructive pulmonary disease. Ann Intern Med, 1987. 106(2): p. 196-204.

21. McGhan, R., et al., Predictors of rehospitalization and death after a severe exacerbation of COPD. Chest, 2007. 132(6): p. 1748-55.

22. Zvezdin, B., et al., A postmortem analysis of major causes of early death in patients hospitalized with COPD exacerbation. Chest, 2009. 136(2): p. 376-80.

23. Hurst, J.R. and J.A. Wedzicha, Management and prevention of chronic obstructive pulmonary disease exacerbations: a state of the art review. BMC Med, 2009. 7: p. 40.

24. Faustini, A., et al., The impact on risk-factor analysis of different mortality outcomes in COPD patients. Eur Respir J, 2008. 32(3): p. 629-36. 
25. Garcia-Aymerich, J., et al., Lung function impairment, COPD hospitalisations and subsequent mortality. Thorax, 2011.

26. Hoogendoorn, M., et al., Case fatality of COPD exacerbations: a meta-analysis and statistical modelling approach. Eur Respir J, 2011. 37(3): p. 508-15.

27. Charlson, M.E., et al., A new method of classifying prognostic comorbidity in longitudinal studies: development and validation. J Chronic Dis, 1987. 40(5): p. 373-83.

28. Brekke, P.H., et al., Troponin T elevation and long-term mortality after chronic obstructive pulmonary disease exacerbation. Eur Respir J, 2008. 31(3): p. 563-570.

29. Fruchter, O. and M. Yigla, Cardiac troponin-I predicts long-term mortality in chronic obstructive pulmonary disease. COPD, 2009. 6(3): p. 155-61.

30. Lieberman, D., et al., Pneumonic vs nonpneumonic acute exacerbations of COPD. Chest, 2002. 122(4): p. 1264-70.

31. Rutten, F.H., et al., Heart failure and chronic obstructive pulmonary disease: An ignored combination? Eur J Heart Fail, 2006. 8(7): p. 706-11.

32. Janssen, D.J., et al., Symptoms, comorbidities, and health care in advanced chronic obstructive pulmonary disease or chronic heart failure. J Palliat Med, 2011. 14(6): p. 735-43.

33. Abroug, F., et al., Association of left-heart dysfunction with severe exacerbation of chronic obstructive pulmonary disease: diagnostic performance of cardiac biomarkers. Am J Respir Crit Care Med, 2006. 174(9): p. 990-6.

34. Tung, R.H., et al., Amino-terminal pro-brain natriuretic peptide for the diagnosis of acute heart failure in patients with previous obstructive airway disease. Ann Emerg Med, 2006. 48(1): p. 66-74.

35. Gray-Donald, K., et al., Nutritional status and mortality in chronic obstructive pulmonary disease. Am J Respir Crit Care Med, 1996. 153(3): p. 961-6.

36. Ai-Ping, C., K.H. Lee, and T.K. Lim, In-hospital and 5-year mortality of patients treated in the ICU for acute exacerbation of COPD: a retrospective study. Chest, 2005. 128(2): p. 518-24.

37. Lim, W.S., et al., Defining community acquired pneumonia severity on presentation to hospital: an international derivation and validation study. Thorax, 2003. 58(5): p. 377-382.

38. Wouters, E.F., Management of severe COPD. Lancet, 2004. 364(9437): p. 883-95.

39. De Angelis, C., et al., Oxygen administration increases plasma digoxin-like substance and renal sodium excretion in chronic hypoxic patients. Am J Nephrol, 1993. 13(3): p. 173-7.

40. Sharkey, R.A., et al., Renal functional reserve in patients with severe chronic obstructive pulmonary disease. Thorax, 1997. 52(5): p. 411-5.

41. Ucgun, I., et al., Relationship of metabolic alkalosis, azotemia and morbidity in patients with chronic obstructive pulmonary disease and hypercapnia. Respiration, 2008. 76(3): p. 270-4.

42. Cazzola, M., et al., Outcomes for COPD pharmacological trials: from lung function to biomarkers. Eur Respir J, 2008. 31(2): p. 416-69.

43. Hallin, R., et al., Nutritional status and long-term mortality in hospitalised patients with chronic obstructive pulmonary disease (COPD). Respir Med, 2007. 101(9): p. 1954-60.

44. Landbo, C., et al., Prognostic value of nutritional status in chronic obstructive pulmonary disease. American Journal of Respiratory and Critical Care Medicine, 1999. 160(6): p. 1856-1861. 



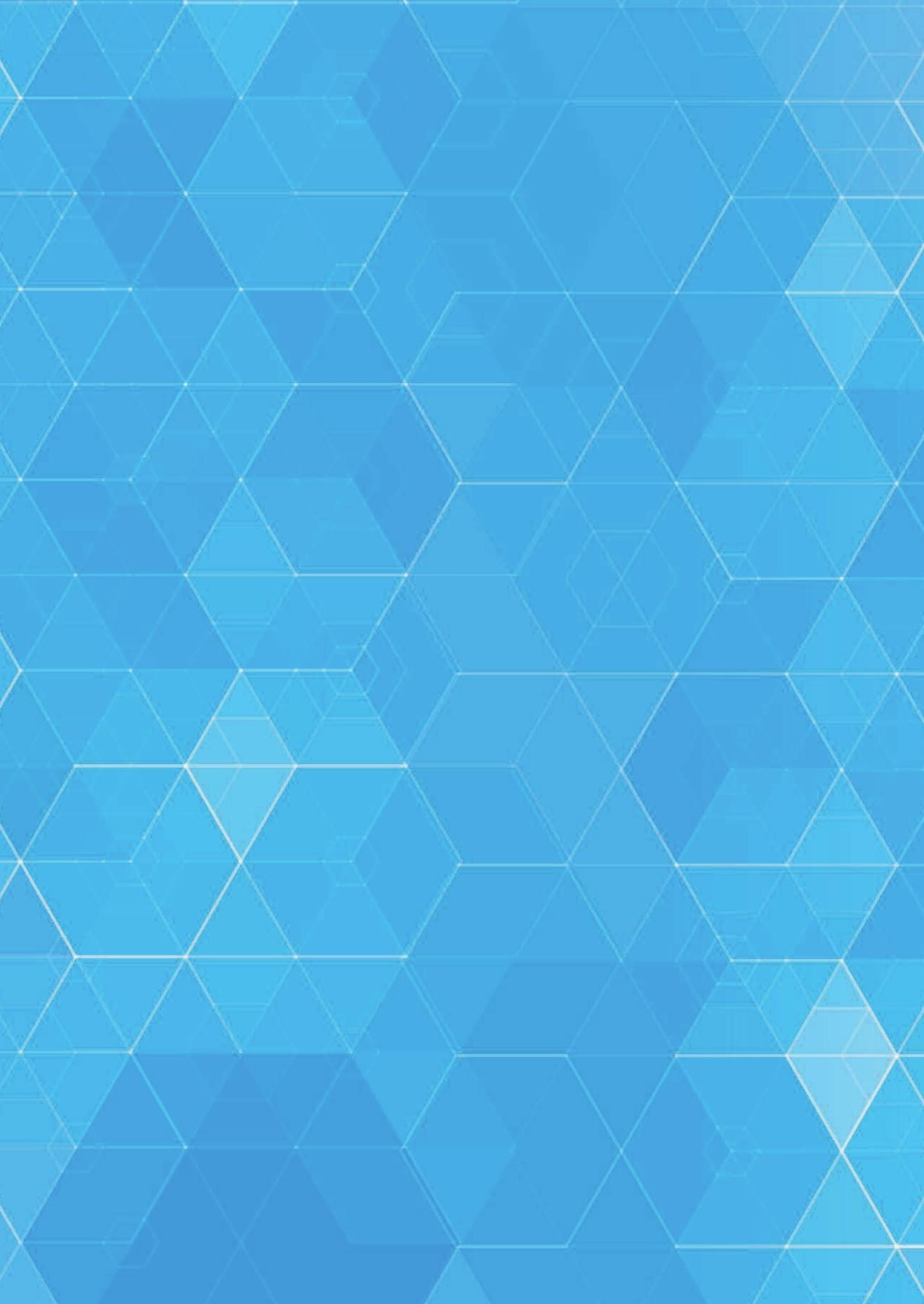




\section{Chapter 5}

Risk stratification for short-term

mortality at hospital admission for acute exacerbation of COPD

Roy T.M. Sprooten*, Gernot G.U. Rohde*, Glenn Lawyer, Wouter T. Leijte, Emiel F.M. Wouters, Frits M.E. Franssen

${ }^{*}$ These authors contributed equally to this work Published in Respirology 2019;24:765-176 


\section{Abstract}

\section{Background}

Exacerbations of COPD (ECOPD) are associated with increased in-hospital and shortterm mortality. Developing an easy-to-use model to predict adverse outcomes will be useful in daily clinical practice and will facilitate management decisions. We aimed to assess mortality rates and potential predictors for short-term mortality after severe ECOPD. Classification and Regression Tree (CART) model was used to identify predictors of adverse outcome.

\section{Methods}

A retrospective observational cohort study, including all patients admitted to Maastricht University Medical Center with ECOPD between June 2011 and December 2014 was performed. The last admission was taken into account and its demographic, clinical and biochemical data were recorded.

\section{Results}

364 hospitalized patients were enrolled. Mean (SD) age was 70.5 (10.2) years, 54.4\% were male and mean $\mathrm{FEV}_{1} 45.2 \%$ (17.7) of predicted. The in-hospital and 90-day mortality were respectively $8.5 \%$ and $16.2 \%$. Independent risk factors for 90-day mortality were: $\mathrm{PaCO}_{2}$ (odds ratio (OR): 1.31; 95\% confidence interval (Cl): 1.00-0.35), age (OR: 1.09; $\mathrm{Cl}$ : 0.06-0.11), $\mathrm{BMI}<18.5 \mathrm{~kg} / \mathrm{m}^{2}$ (OR: $2.72 ; 95 \% \mathrm{Cl}: 0.53-1.47$ ) and previous admission for ECOPD in last two years (OR: $1.29 ; 95 \% \mathrm{Cl}:-0.14-0.65$ ). The CART model selected $\mathrm{PaCO}_{2} \geq 9.1 \mathrm{kPa}$, age $>80$ years, $\mathrm{BMI}<18.5 \mathrm{~kg} / \mathrm{m}^{2}$ and previous admission for ECOPD as the most discriminatory factors.

\section{Conclusions}

According CART analysis, high $\mathrm{PaCO}_{2}$ and age, low BMI and previous admission for ECOPD in last two years were the strongest predictors of 90-day mortality in patients with severe ECOPD. In absence of any of these factors, no patients died, suggesting that this model indeed enables risk stratification. 


\section{Introduction}

Acute exacerbations of chronic obstructive pulmonary disease (ECOPD) play a pivotal role in the natural course of morbidity associated with this disease as they contribute to declines in lung function ${ }^{1}$, health status ${ }^{2}$, and physical activity level. ${ }^{3}$ Moreover, exacerbations are the major cause of hospitalisations in patients with chronic obstructive pulmonary disease (COPD), which are among the key drivers of the economic burden of the disease ${ }^{4}$ and are associated with substantial mortality risk. ${ }^{5}$ Previous studies showed a broad range of mortality rates after ECOPD with an inhospital mortality of $2.9-10.4 \%, 90$-day mortality of $4.8-13.9 \%$ and a 1 -year mortality of 23.0-27.7\%. ${ }^{6-12}$ Predicting in-hospital and short-term mortality, using clinical, biological and biochemical factors strongly associated with high risk of adverse outcome is crucial for clinicians in a hospital department or emergency room (ER) to make decisions regarding management of ECOPD, setting of treatment (at home versus in-hospital), length of hospital stay and advance care planning. Several prognostic tools for stable COPD are available, including $B O D E$ and $A D O^{13-15}$, while other studies investigated potential factors associated with high in-hospital and short-term mortality after hospital admission for ECOPD. Various predictive tools were introduced ${ }^{8,12,16-19}$, although some were not specifically developed for COPD $^{16,17}$, used readmissions and/or mortality at 90 days as combined endpoints ${ }^{12,18}$ or focussed on in-hospital mortality only. ${ }^{8}$ While these studies used linear and additive models to predict outcomes, the use of a classification and regression tree (CART) could be a helpful alternative approach. CART results in a decision rule with a hierarchical sequential structure which can be easily used in daily clinical practice. To our knowledge two studies used CART analysis to predict mortality in COPD $^{20,21}$ of which one identified several predictors for 30 day mortality after attending ER. ${ }^{21}$

The aims of the present study were to (i) assess the short-term (in-hospital and 90-day) mortality rate of ECOPD, (ii) identify clinically available predictors for 90-day mortality risk in patients admitted for severe ECOPD and (iii) to construct an easy, feasible and comprehensive predictive algorithm for 90-day mortality in this patient population.

\section{Methods}

We conducted a retrospective, observational cohort study at the department of respiratory medicine of Maastricht University Medical Center (MUMC) in Maastricht, the Netherlands. Clinical data of eligible patients who had been hospitalized for ECOPD 
on our pulmonary ward between 01-06-2011 and 31-12-2014 were included. Patients were initially identified on the basis of the ICD-9 (International Statistical Classification of Diseases and Related Health Problems) and ICD-10 (2014) codes for COPD (J42-44) and 'acute respiratory symptoms or pneumonia' (ICD-9 786.0-9 and ICD-10 J12-16) in the hospital electronic database. The index admission was the last admission during the study period. The inclusion criteria were: (i) severe ECOPD according to the GOLD definition $^{22}$ and (ii) confirmation of obstructive lung function by a ratio of postbronchodilator forced expiratory volume in 1 second to forced vital capacity (FEV $1 / F V C$ ) $<70 \%{ }^{22}$ in the medical record of the patient. Exclusion criteria were: age $<40$ years, a previous diagnosis with asthma, active pulmonary malignancy, follow-up in another hospital and primary reason for hospitalization other than ECOPD. Patients admitted for ECOPD and consolidation on chest X-ray were not excluded. This study was conducted according to the Declaration of Helsinki $\left(59^{\text {nd }}\right.$ WMA General Assembly, Seoul, October 2008) and Good Clinical Practice guidelines. According the Dutch law, for this retrospective study informed consent was not required. Because we used deidentified data in this retrospective study ethical approval was not necessary according the local ethical committee of the MUMC (METC 13-4-041).

After the descriptive analyses, univariate analyses were performed using independenttests, $\mathrm{X}^{2}$-tests or fisher's exact tests as appropriate to compare the characteristics of patients who survived the hospital admission and 90 days or who died during hospital stay and within 90-days of follow-up. Clinically relevant factors with a p-value $<0.05$ in univariate analysis were included in the multivariate analysis. Clinically relevant and significant predictors were used as input to the CART model. ${ }^{23}$ (See Appendix S5.1 in Supplementary information)

\section{Results}

The flow chart for patient selection is shown in Figure 5.1. The clinical characteristics of the study population is depicted in Table 5.1. Vital signs and laboratory results at the time of presentation at the ER are presented in Table 5.2. 
Figure 5.1 Flowchart of cohort selection.

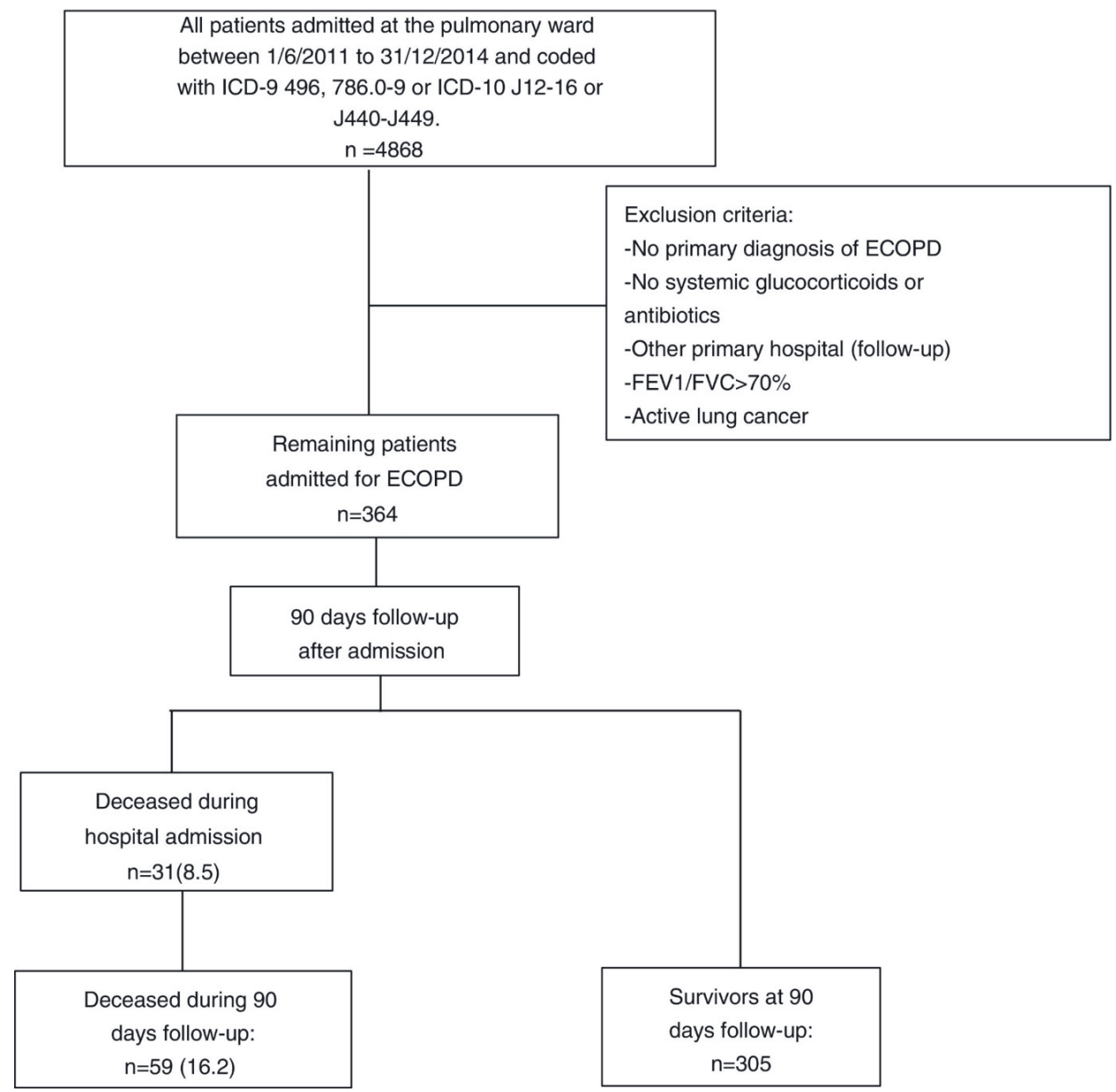

Data presented as $\mathrm{n}(\%)$. Abbreviations: ICD: International statistical classification of diseases and related health problems; ECOPD: exacerbation of chronic obstructive pulmonary disease; $\mathrm{FEV}_{1}$ : forced expiratory volume in 1s; FVC: forced vital capacity. 
Table 5.1 Demographic and prior health-care utilization characteristics of patients hospitalized for ECOPD, stratified by in-hospital mortality as outcome.

\begin{tabular}{|c|c|c|c|c|}
\hline Characteristics (n) & $\begin{array}{c}\text { All } \\
(n=364)\end{array}$ & $\begin{array}{c}\text { Alive } \\
(n=333) \\
\end{array}$ & $\begin{array}{c}\text { Deceased } \\
(n=31)\end{array}$ & p-value \\
\hline Age (years) & $70.52 \pm 10.19$ & $70.11 \pm 10.16$ & $74.84 \pm 9.62$ & 0.013 \\
\hline Age $\geq 80$ (years) & $79(21.7)$ & $53(17.4)$ & $26(44.1)$ & $<0.0001$ \\
\hline Gender, Male (364) & $169(46.4)$ & $154(46.2)$ & $15(48.4)$ & $0.819^{\#}$ \\
\hline \multicolumn{5}{|l|}{ Smoking status (357/326/31) } \\
\hline Current smoker & $132(37.0)$ & $124(38.0)$ & $8(25.8)$ & $0.178^{\#}$ \\
\hline \multicolumn{5}{|l|}{ Supported living and extra care } \\
\hline Extra care $(349 / 321 / 28)$ & $185(53.0)$ & $164(51.1)$ & $21(75.0)$ & $0.015^{\#}$ \\
\hline \multicolumn{5}{|l|}{ Cohabiting (332/305/27) } \\
\hline Yes & $196(59.0)$ & $180(59.0)$ & $16(59.3)$ & $0.980^{\#}$ \\
\hline \multicolumn{5}{|l|}{ Residence status(351/322/29) } \\
\hline Own home & $292(83.2)$ & $274(85.1)$ & $18(62.1)$ & $0.006^{\#}$ \\
\hline Retirement home & $47(13.4)$ & $38(11.8)$ & $9(31.0)$ & \\
\hline Other & $12(3.4)$ & $10(3.1)$ & $2(6.9)$ & \\
\hline \multicolumn{5}{|l|}{ Policy restrictions } \\
\hline Yes $(364 / 335 / 31)$ & $92(25.3)$ & $74(22.2)$ & $18(58.1)$ & $<0.001^{\#}$ \\
\hline \multicolumn{5}{|l|}{ Gold-stage (364/333/31) } \\
\hline 1: $\mathrm{FEV}_{1} \geq 80 \%$ & $10(2.7)$ & $9(2.7)$ & $1(3.2)$ & $0.428^{\#}$ \\
\hline $2: 50 \% \leq \mathrm{FEV}_{1}<80 \%$ & 119 (32.7) & $113(33.9)$ & $6(19.4)$ & \\
\hline $3: 30 \% \leq \mathrm{FEV}_{1}<50 \%$ & $170(46.7)$ & 153 (45.9) & $17(54.8)$ & \\
\hline 4: $\mathrm{FEV}_{1}<30 \%$ & $65 \quad(17.0)$ & $58(17.4)$ & $7(22.6)$ & \\
\hline \multicolumn{5}{|l|}{ Lung function } \\
\hline $\mathrm{FEV}_{1}$ liters $(364 / 333 / 31)$ & $1.05 \pm 0.44$ & $1.06 \pm 0.44$ & $0.87 \pm 0.32$ & 0.003 \\
\hline $\mathrm{FEV}_{1}$ pred\% $(364 / 333 / 31)$ & $45.19 \pm 16.76$ & $45.66 \pm 16.78$ & $40.14 \pm 15.83$ & 0.079 \\
\hline $\mathrm{FEV}_{1} / \mathrm{VC}(364 / 333 / 31)$ & $41.56 \pm 11.82$ & $41.93 \pm 11.96$ & $37.51 \pm 9.44$ & 0.046 \\
\hline \multicolumn{5}{|l|}{ Body box } \\
\hline RV/TLC pred\% (227/206/21) & $146.29 \pm 28.64$ & $146.14 \pm 28.46$ & $147.75 \pm 31.04$ & 0.81 \\
\hline \multicolumn{5}{|l|}{ Diffusion } \\
\hline DLCO/SB pred\% (206/191/15) & $46.72 \pm 17.87$ & $47.52 \pm 17.97$ & $36.74 \pm 13.44$ & 0.024 \\
\hline \multicolumn{5}{|l|}{ Previous admission } \\
\hline Admission ever (364/333/31) & $204(56.0)$ & $178(53.5)$ & $26(12.7)$ & $0.001^{\#}$ \\
\hline \multicolumn{5}{|l|}{ Admissions last 2 year } \\
\hline An admission (364/305/59) & $160(44.0)$ & $140(42.0)$ & $21(67.7)$ & $0.008^{\#}$ \\
\hline-0 & $203(55.8)$ & $193(58.0)$ & $10(32.3)$ & $0.134^{\#}$ \\
\hline-1 & $89(24.5)$ & $76(22.8)$ & $13(41.9)$ & \\
\hline-2 & 35 (9.6) & $31(9.3)$ & 4 (12.9) & \\
\hline$-\geq 3$ & $37(10.2)$ & $27(8.1)$ & 4 (12.9) & \\
\hline \multicolumn{5}{|l|}{ An admission } \\
\hline - non-frequent 0-1 & $292(80.2)$ & $269(80.8)$ & $23(74.2)$ & $0.379^{\#}$ \\
\hline - frequent & $72(19.8)$ & $64(19.2)$ & $8(25.8)$ & \\
\hline Acute NPPV & $44(12.1)$ & $38(11.4)$ & $6(19.47)$ & $0.194^{\#}$ \\
\hline \multicolumn{5}{|l|}{ Comorbidities } \\
\hline Charlson's index & $2.22 \pm 1.44$ & $2.23 \pm 1.46$ & $2.10 \pm 1.25$ & 0.61 \\
\hline \multicolumn{5}{|l|}{$\mathrm{CCl}$ category } \\
\hline $\mathrm{CCl} 1-2$ points & 245 (67.3) & $223(67.0)$ & $22(71.0)$ & $0.650^{\#}$ \\
\hline $\mathrm{CCl} \geq 3$ points & $119(32.7)$ & $110(33.0)$ & $9(29.0)$ & \\
\hline
\end{tabular}


Table 5.1 (continued)

\begin{tabular}{lcccc}
\hline Characteristics $(\mathbf{n})$ & $\begin{array}{c}\text { All } \\
(\mathbf{n}=\mathbf{3 6 4})\end{array}$ & $\begin{array}{c}\text { Alive } \\
(\mathbf{n}=\mathbf{3 3 3})\end{array}$ & $\begin{array}{c}\text { Deceased } \\
(\mathbf{n}=\mathbf{3 1})\end{array}$ & p-value \\
\hline Home treatment & & & & \\
None (359/328/31) & $16(4.5)$ & $16(4.9)$ & $0(0.0)$ & $0.208^{\#}$ \\
ICS (351/306/29) & $276(78.6)$ & $250(81.7)$ & $26(89.7)$ & $0.282^{\#}$ \\
SABA (353/309/30) & $206(58.4)$ & $183(59.2)$ & $23(76.7)$ & $0.620^{\#}$ \\
LABA (352/307/29) & $291(82.7)$ & $266(86.6)$ & $25(86.2)$ & $0.947^{\#}$ \\
SAMA (350/306/28) & $93(26.6)$ & $80(26.1)$ & $13(46.4)$ & $0.022^{\#}$ \\
LAMA (353/307/30) & $265(75.1)$ & $241(78.5)$ & $24(80.0)$ & $0.848^{\#}$ \\
Prednisolone*(352/308/29) & $37(10.5)$ & $31(10.1)$ & $6(20.7)$ & $0.080^{\#}$ \\
Antibiotics* (351/307/29) & $39(11.1)$ & $36(11.7)$ & $3(10.3)$ & $0.825^{\#}$ \\
LTOT (355/309/31) & $93(26.2)$ & $78(25.2)$ & $15(48.4)$ & $0.006^{\#}$ \\
CPAP (352/308/30) & $13(3.7)$ & $13(4.2)$ & $0(0)$ & $0.251^{\#}$ \\
cNIV (352/307/30) & $14(4.0)$ & $13(4.2)$ & $1(3.3)$ & $0.813^{\#}$ \\
\hline
\end{tabular}

Data presented as $\mathrm{n}(\%)$ or medium with standard deviation depicted after this symbol \pm , unless otherwise stated. "Pearson Chi square, *maintenance therapy. Abbreviations: FEV ${ }_{1}$ : forced expiratory volume in 1 second, $\mathrm{FEV}_{1} / \mathrm{FVC}$ : ratio $\mathrm{FEV}_{1} / \mathrm{FVC}$, RV/TLC: ratio residual volume and total lung capacity, TLCO: diffusion capacity of carbon monoxide, NIV: non-invasive positive pressure ventilation, cNIV: chronic NIV, CCl: Charlson's comorbidity index, ICS: inhalation corticosteroids, SABA: short acting beta agonist, LABA: long acting beta agonist, SAMA: short acting muscarinic antagonist, LAMA: long acting muscarinic antagonist, LTOT: long term oxygen therapy, CPAP: continuous positive pressure ventilation, pred\%: percentage of predicted value.

Table 5.2 Measurements of patients at emergency room (ER) presentation and hospitalized for ECOPD, stratified by in-hospital mortality as outcome.

\begin{tabular}{|c|c|c|c|c|}
\hline Characteristics (n) & $\begin{array}{c}\text { All } \\
(n=364)\end{array}$ & $\begin{array}{c}\text { Alive } \\
(n=333)\end{array}$ & $\begin{array}{c}\text { Deceased } \\
(n=31)\end{array}$ & p-value \\
\hline \multicolumn{5}{|l|}{ Vital signs } \\
\hline Temperature, C (323/300/23) & $37.3 \pm 0.99$ & $37.31 \pm 0.99$ & $37.13 \pm 0.91$ & 0.394 \\
\hline Systolic BP, mmHg(355/326/29) & $141 \pm 26.38$ & $140.4 \pm 25.78$ & $143.0 \pm 32.88$ & 0.616 \\
\hline Diastolic BP, mmHg & $77.0 \pm 16.28$ & $76.6 \pm 15.97$ & $79.0 \pm 19.65$ & 0.437 \\
\hline Pulse, beats/min $(357 / 328 / 29)$ & $101.0 \pm 20.97$ & $99.6 \pm 20.46$ & $111.0 \pm 24.13$ & 0.005 \\
\hline Pulse $>100 / \mathrm{min}$ & $148(41.5)$ & $130(39.4)$ & $18(62.1)$ & $0.019^{\#}$ \\
\hline $\mathrm{SpO}_{2} \%(358 / 328 / 30)$ & $91 \pm 6.26$ & $90.9 \pm 5.51$ & $87.7 \pm 11.26$ & 0.136 \\
\hline $\mathrm{SpO} 2<90 \%$ & $155(43.3)$ & $137(42.8)$ & $18(60.0)$ & $0.540^{\#}$ \\
\hline Extra $\mathrm{FiO}_{2}(335 / 283 / 52)$ & $206(61.5)$ & $183(59.6)$ & $23(82.1)$ & $0.019^{\#}$ \\
\hline \multicolumn{5}{|l|}{ Physical examination } \\
\hline Respiratory rate/min $(173 / 162 / 11)$ & $25 \pm 6.13$ & $24.9 \pm 5.90$ & $27.6 \pm 8.86$ & 0.355 \\
\hline Confused $(27 / 21 / 6)$ & $8(29.6)$ & $6(28.4)$ & $2(33.3)$ & $0.822^{\#}$ \\
\hline Use of accessory muscles (34/30/4) & $30(88.2)$ & $26(86.7)$ & $4(100)$ & $0.437^{\#}$ \\
\hline Elevated CVP (119/109/10) & $20(16.8)$ & $17(15.6)$ & $3(30.0)$ & $0.244^{\#}$ \\
\hline Peripheral edema $(307 / 282 / 25)$ & $88(28.7)$ & $81(28.7)$ & $7(28.0)$ & $0.939^{\#}$ \\
\hline Wheezing $(360 / 329 / 31)$ & $212(58.9)$ & $195(59.3)$ & $17(54.8)$ & $0.632^{\#}$ \\
\hline Crackles $(360 / 229 / 31)$ & 115 (31.9) & $107(32.5)$ & $8(25.8)$ & $0.443^{\#}$ \\
\hline BMI, kg/m² (353/327/26) & $25.00 \pm 6.39$ & $25.05 \pm 6.43$ & $24.26 \pm 5.95$ & 0.451 \\
\hline Underweight $\mathrm{BMI}<18.5 \mathrm{~kg} / \mathrm{m}^{2}$ & $52(14.7)$ & $48(14.7)$ & $4(15.4)$ & $0.552^{\#}$ \\
\hline Overweight $\mathrm{BMI}>25 \mathrm{~kg} / \mathrm{m}^{2}$ & $151(42.8)$ & $133(44)$ & $8(30.7)$ & $0.244^{\#}$ \\
\hline
\end{tabular}


Table 5.2 (continued)

\begin{tabular}{|c|c|c|c|c|}
\hline Characteristics (n) & $\begin{array}{c}\text { All } \\
(n=364)\end{array}$ & $\begin{array}{c}\text { Alive } \\
(n=333)\end{array}$ & $\begin{array}{c}\text { Deceased } \\
(n=31)\end{array}$ & p-value \\
\hline \multicolumn{5}{|l|}{ Arterial blood gas. } \\
\hline $\mathrm{pH}(362 / 331 / 31)$ & $7.37 \pm 0.088$ & $7.38 \pm 0.084$ & $7.31 \pm 0.105$ & 0.001 \\
\hline$<7.25$ & $39(10.8)$ & $29(8.8)$ & $10(32.3)$ & $<0.001^{\#}$ \\
\hline $7.25-7.35$ & $80(22.1)$ & $72(21.8)$ & $8(25.8)$ & \\
\hline$>7.35$ & $243(67.1)$ & $230(69.5)$ & 13 (41.9) & \\
\hline $\mathrm{PaCO}_{2} \mathrm{kPa}(362 / 331 / 31)$ & $6.98 \pm 3.17$ & $6.80 \pm 2.21$ & $8.94 \pm 3.24$ & 0.001 \\
\hline $\mathrm{PaCO}_{2} \geq 6.0 \mathrm{kPa}$ & $206(56.9)$ & $181(54.7)$ & $25(12.1)$ & $0.005^{\#}$ \\
\hline $\mathrm{PaCO}_{2} \geq 7.0 \mathrm{kPa}$ & $154(42.5)$ & $131(39.6)$ & $23(74.2)$ & $<0.001^{\#}$ \\
\hline $\mathrm{PaCO}_{2}>9.1 \mathrm{kPa}$ & $61(16.9)$ & 46 (13.9) & $15(48.4)$ & $<0.001^{\#}$ \\
\hline $\mathrm{HCO}_{3} \geq 27.0 \mathrm{mmol} / \mathrm{L}(358 / 327 / 31)$ & $222(62)$ & $198(60.6)$ & $24(77.4)$ & $0.064^{\#}$ \\
\hline $\mathrm{HCO}_{3}^{-} \mathrm{mmol} / \mathrm{L}$ & $29.50 \pm 6.32$ & $29.23 \pm 6.13$ & $32.29 \pm 7.57$ & 0.010 \\
\hline Base excess $(358 / 327 / 31)$ & $3.17 \pm 5.04$ & $3.01 \pm 4.91$ & $3.94 \pm 6.34$ & 0.374 \\
\hline$<-2.5$ & $40(10.7)$ & $36(11.0)$ & 4 (12.9) & $0.121^{\#}$ \\
\hline$-2.5-2.5$ & $142(39.7)$ & $135(41.3)$ & $7(22.6)$ & \\
\hline$>2.5$ & $176(49.2)$ & $156(47.7)$ & $20(64.5)$ & \\
\hline $\mathrm{PaO}_{2} \mathrm{kPa}(358 / 327 / 31)$ & $8.15 \pm 3.17$ & $7.98 \pm 2.78$ & $9.96 \pm 5.64$ & 0.620 \\
\hline $\mathrm{SaO}_{2} \%(357 / 326 / 31)$ & $86.04 \pm 8.16$ & $85.99 \pm 7.79$ & $86.61 \pm 11.51$ & 0.686 \\
\hline $\left.\mathrm{SaO}_{2}<90 \%\right)$ & $155(43.3)$ & $126(42.0)$ & $29(50.0)$ & $0.260^{\#}$ \\
\hline Extra $\mathrm{FiO}_{2}(314 / 266 / 48)$ & $142(45.2)$ & $102(38.2)$ & $40(85.1)$ & $<0.001^{\#}$ \\
\hline $\mathrm{PaO}_{2}<7.5 \mathrm{kPa}(358 / 327 / 31)$ & $178(49.7)$ & $167(51.1)$ & $11(35.5)$ & $0.097^{\#}$ \\
\hline \multicolumn{5}{|l|}{ Laboratory results } \\
\hline Hemoglobin mmol/L (354/323/31) & $8.38 \pm 1.21$ & $8.40 \pm 1.23$ & $8.18 \pm 1.05$ & 0.864 \\
\hline Low $<8.2$ & $151(42.7)$ & $136(42.1)$ & $15(48.4)$ & $0.707^{\#}$ \\
\hline Normal $8.2-11.0$ & $200(56.5)$ & $184(57.0)$ & $16(51.6)$ & \\
\hline High $>11.0$ & $3(0.8 \%)$ & $3(0.9)$ & $0(0.0)$ & \\
\hline Hematocrit $(188 / 171 / 17)$ & $0.42 \pm 0.06$ & $0.42 \pm 0.06$ & $0.42 \pm 0.05$ & 0.864 \\
\hline WBC $10^{9} / L(362 / 331 / 31$ & $13.40 \pm 5.98$ & $13.28 \pm 5.85$ & $14.64 \pm 7.22$ & 0.229 \\
\hline$W B C>1110^{9} / L$ & $218(60.2)$ & $198(59.8)$ & $20(64.5)$ & $0.609^{\#}$ \\
\hline Eosinophilia \% (138/124/14) & $1.04 \pm 1.98$ & $1.14 \pm 2.06$ & $0.14 \pm 0.36$ & $<0.00$ \\
\hline $\mathrm{Na} \mathrm{mmol} / \mathrm{L}(360 / 329 / 31)$ & $137.27 \pm 4.87$ & $137.16 \pm 4.91$ & $138.39 \pm 4.24$ & 0.182 \\
\hline $\mathrm{Na}<135 \mathrm{mmol} / \mathrm{L}$ & $87(24.2)$ & $80(24.3)$ & $7(22.6)$ & $0.829^{\#}$ \\
\hline Urea mmol/L (347/317/30) & $7.72 \pm 5.02$ & $7.36 \pm 4.50$ & $11.50 \pm 8.00$ & 0.009 \\
\hline Urea $\geq 8 \mathrm{mmol} / \mathrm{L}$ & $114(32.9)$ & $97(30.6)$ & $17(56.7)$ & $0.004^{\#}$ \\
\hline Creatinine mmol/L (357/326/31) & $93.89 \pm 62.96$ & $92.33 \pm 59.29$ & $110.29 \pm 93.07$ & 0.300 \\
\hline Creatinine $>115 \mu \mathrm{mol} / \mathrm{L}$ & $73(20.4)$ & 65 (19.9) & $8(25.8)$ & $0.439^{\#}$ \\
\hline ASAT U/L(159/139/20) & $62.52 \pm 341.22$ & $67.00 \pm 364.78$ & $31.35 \pm 23.96$ & 0.664 \\
\hline ASAT $>35 \mathrm{U} / \mathrm{L}$ & $49(30.8)$ & $44(31.7)$ & $5(25.0)$ & $0.547^{\#}$ \\
\hline ALAT U/L (157/140/17) & $43.34 \pm 157.04$ & $45.36 \pm 166.08$ & $26.71 \pm 22.56$ & 0.645 \\
\hline ALAT $>45 \mathrm{U} / \mathrm{L}$ & $20(12.7)$ & $19(13.6)$ & $1(5.9)$ & $0.328^{\#}$ \\
\hline LDH U/L (118/103/15) & $310.83 \pm 322.32$ & $314.32 \pm 342.80$ & $286.87 \pm 106.36$ & 0.759 \\
\hline $\mathrm{LDH} \geq 500 \mathrm{U} / \mathrm{L}$ & $8(6.8)$ & $7(6.8)$ & $1(6.7)$ & 1.00 \\
\hline Troponin $>14 \mathrm{ng} / \mathrm{L}$ & $89(24.5)$ & $81(24.3)$ & $8(25.8)$ & $0.854^{\#}$ \\
\hline NT-proBNP pmol/L (139/124/15) & $435 \pm 636.58$ & $428.47 \pm 658.38$ & $494.55 \pm 0.016$ & 0.706 \\
\hline NT-proBNP $\geq 500 \mathrm{pmol} / \mathrm{L}$ & $31(22.3)$ & $25(20.2)$ & $6(40.0)$ & $0.081^{\#}$ \\
\hline Albumin g/L (9/8/1) & $32.39 \pm 4.20$ & $31.98 \pm 4.29$ & 35.7 & 0.000 \\
\hline Albumin <35 g/L (9/8/1) & $6(66.7)$ & $6(75)$ & $0(0)$ & $0.333^{\#}$ \\
\hline
\end{tabular}


Table 5.2 (continued)

\begin{tabular}{|c|c|c|c|c|}
\hline Characteristics (n) & $\begin{array}{c}\text { All } \\
(n=364)\end{array}$ & $\begin{array}{c}\text { Alive } \\
(n=333)\end{array}$ & $\begin{array}{c}\text { Deceased } \\
(n=31)\end{array}$ & p-value \\
\hline CRP mg/L (361/330/31) & $70.96 \pm 87.35$ & $70.31 \pm 86.28$ & $77.92 \pm 99.30$ & 0.643 \\
\hline$<50$ & $212(58.7)$ & $193(58.5)$ & $19(61.3)$ & $0.410^{\#}$ \\
\hline $50-200$ & $111(30.7)$ & $104(31.5)$ & $7(22.6)$ & \\
\hline$>200$ & $38(10.5)$ & $33(10.0)$ & $5(16.1)$ & \\
\hline Glucose $\mathrm{mmol} / \mathrm{L}(321 / 292 / 29)$ & $7.86 \pm 2.96$ & $7.81 \pm 2.93$ & $8.42 \pm 3.20$ & 0.291 \\
\hline Glucose $\geq 11 \mathrm{mmol} / \mathrm{L}$ & $42(13.1)$ & $38(13.0)$ & $4(13.8 \%)$ & $0.906^{\#}$ \\
\hline \multicolumn{5}{|l|}{ Chest X-ray $(363 / 332 / 31)$} \\
\hline Consolidation & $107(29.5)$ & $93(28.0)$ & $14(45.2)$ & $0.045^{\#}$ \\
\hline Signs of congestion & $52(14.3)$ & $49(18.8)$ & $3(9.7)$ & $0.440^{\#}$ \\
\hline \multicolumn{5}{|l|}{ ECG $(329 / 302 / 27)$} \\
\hline Atrial fibrillation & $43(13.1)$ & $34(11.3)$ & $9(33.3)$ & $0.001^{\#}$ \\
\hline Signs of ischemia & $22(6.7)$ & $21(7.0)$ & $1(3.7)$ & $0.517^{\#}$ \\
\hline
\end{tabular}

Data presented as $\mathrm{n}(\%)$ or medium with standard deviation depicted after \pm , unless otherwise stated. \#Pearson Chi square. Abbreviations: $\mathrm{BP}$ : blood pressure, $\mathrm{SpO}_{2}$ : oxygen saturation measured by pulse oximetry, $\mathrm{FiO}_{2}$ : fraction of inspired oxygen, CVP: central venous pressure, BMI: body mass index, SaO $\mathrm{O}_{2}$ : oxygen saturation measured in arterial blood gas, WBC: white blood cell, Na: sodium, ASAT: aminotransferase, ALAT: alanineaminotranferase, LDH: lactic acid dehydrogenase, NT-proBNP: brain natriuremic peptide, CRP: Creactive protein, ECG: electrocardiogram, ABG: arterial blood gas, ER: emergency room. kPa: kilopascal, $\mathrm{mmol} / \mathrm{l}$ : millimol per liter, $\mu \mathrm{mol} / \mathrm{L}$ : micromol per liter, pmol/L: picomol per liter, U/L: units per liter, $\mathrm{ng} / \mathrm{L}$ : nanogram per liter, \%: percentage.

\section{Follow-up and mortality}

During the 90 days follow-up 59 (16.2\%) patients died of which 30 (8.2\%) died inhospital and $38(10.4 \%)$ patients were dead at 30 days after admission. The mean length of hospital stay was $9.0 \pm 7.6$ days. During the admission mechanical ventilation was required in 115 (31.5\% form the study population) patients, of these 110 (30.2\%) patients needed non-invasive ventilation (NIV) of whom in 7 patients NIV failed and switched to invasive ventilation (IV). In 8 patients IV was initially started of whom 3 patients were weaned in later phase with NIV. In total 15 (4.1\%) patients were treated with IV. Of the patients who required mechanical ventilation, thirty-two (27.8\%) died during 90-days follow-up and this group had a significantly increased 90-day mortality rate compared to patients not requiring mechanical ventilation (10.8\%) (Pearson$\left.x^{2}: 16.706 ; p<0.0001\right)$. The in-hospital mortality in patients using NIV $(n=110)$ was 21 (19\%) patients and was significant higher than in patients not using mechanical ventilation (Pearson- $\mathrm{x}^{2}$ : 24.535, $\left.\mathrm{p}<0001\right)$. Twenty-six patients $(7.8 \%$ of discharged population), had an early readmission for respiratory problems within 90-days followup. Of these patients 7 died after their readmission. 


\section{Univariate and multivariate analysis}

Patients who died in hospital were older, more care-dependent, had more severe lung function impairment, more often a restricted policy towards cardiopulmonary resuscitation and invasive mechanical ventilation (Table 5.1), significantly more usage of Long-term oxygen treatment (LTOT), more often previous admission in the last two years. On presentation at ER, non-survivors were characterized by increased pulse rate, increased need for supplemental oxygen, higher levels of $\mathrm{PaCO}_{2}$ retention, higher level of urea, lower eosinophil counts, increased frequency of consolidation and signs of cardiovascular instability with atrial fibrillation. The same outcomes were observed in patients that died within 90 days (Tables 5.1-5.4). The univariate analysis in the dataset suggested 9 clinically relevant and nominally significant candidate coefficients: age (continuous variable), Body mass index (BMI) (categorical, $<18.5 \mathrm{~kg} / \mathrm{m}^{2}$ ), infiltration (categorical), previous admission (categorical), LTOT (categorical), supported living (categorical "no extra care", "extra care"), $\mathrm{PaCO}_{2}$ (continuous), Gold stage for airflow limitation (categorical), Urea (continuous). The associated odds ratios and $p$-values for all covariates are shown in Table S5.1 in supplementary information.

\section{Classification tree analysis}

CART analysis using the full data selected four variables as having discriminant power: $\mathrm{PaCO}_{2}$, age, $\mathrm{BMI}$ and prior admission last two years. These discriminant variables and cut-off points were supported by the randomized subsampling. The decision tree was built from full data from 306 patients. The tree first splits patients based on $\mathrm{PaCO}_{2}$ levels. Those with lower $\mathrm{PaCO}_{2}$ are then split on age, and younger patients are again split on BMI and last split on prior admissions (last two years, see Figure 5.2). Patients were thus placed into one of five categories. Patients are initially divided based on $\mathrm{PaCO}_{2}$ level of $9.1 \mathrm{kPa}$. Of the 58 patients with $\mathrm{PaCO}_{2}$ higher than this threshold, $34.5 \%$ $(n=20)$ had 90-day mortality. For the remaining patients, i.e. those with $\mathrm{PaCO}_{2}$ equal to or less than $9.1 \mathrm{kPa}$, the next most discriminant factor was age greater than 80 years old $(n=57)$. In this category $16(28.1 \%)$ patients were dead after 90-days follow-up. For the remaining patients, i.e. those with $\mathrm{PaCO}_{2}$ equal to or less than $9.1 \mathrm{kPa}$ and age less than 80 years, the most discriminant factor was $\mathrm{BMI}<18.5 \mathrm{~kg} / \mathrm{m}^{2}$. Of the 30 patients in this category, $20 \%(n=6)$ died within 90 days. Prior admissions in last two years was the next most discriminating factor. Of the 70 patients in group II $8.6 \%(n=6)$ died within 90 days. Of the remaining 90 patients $\left(\mathrm{PaCO}_{2}\right.$ less than $9.1 \mathrm{kPa}$, aged under 80 years, $\mathrm{BMI}$ $>18.5 \mathrm{~kg} / \mathrm{m}^{2}$ and no prior admission), no one died within 90 days (Figure 5.2 and supplementary information, Table S5.2). The remaining variables were not used by the tree. Figure 5.2 gives a visual representation of the tree. In the CART model the most 
predictive factors are level of $\mathrm{PaCO}_{2}$, Age, $\mathrm{BMI}$ and prior admissions for ECOPD in last two years. (See Appendix S5.2 in supplementary material)

Table 5.3 Demographic and prior health-care utilization characteristics of patients hospitalized for ECOPD, stratified by 90 -days outcome.

\begin{tabular}{|c|c|c|c|}
\hline Characteristics (n) & $\begin{array}{c}\text { Alive } \\
(n=305)\end{array}$ & $\begin{array}{c}\text { Deceased } \\
(n=59)\end{array}$ & $p$-value \\
\hline Age (years) & $69.45 \pm 10.07$ & $75.98 \pm 8.93$ & $<0.001$ \\
\hline Age $\geq 80$ (years) & $53(17.4)$ & $26(44.1)$ & $<0.0001^{\#}$ \\
\hline Gender, Male (364) & $139(45.6)$ & $30(50.8)$ & $0.457^{\#}$ \\
\hline \multicolumn{4}{|l|}{ Smoking status (357/299/57) } \\
\hline Current smoker & $114(38.3)$ & $18(30.5 \%)$ & $0.306^{\#}$ \\
\hline \multicolumn{4}{|l|}{ Supported living and extra care } \\
\hline Extra care $(349 / 296 / 53)$ & $145(49.0)$ & $40(75.5)$ & $<0.0001^{\#}$ \\
\hline \multicolumn{4}{|l|}{ Cohabiting } \\
\hline Yes & $169(60.1)$ & $27(52.9)$ & 0.336 \\
\hline \multicolumn{4}{|l|}{ Residence status } \\
\hline Own home & $258(87.2)$ & $34(61.8)$ & $<0.001^{\#}$ \\
\hline Retirement home & $30(10.1)$ & $17(30.9)$ & \\
\hline Other & $9(3.0)$ & $3(5.6)$ & \\
\hline \multicolumn{4}{|l|}{ Policy restrictions } \\
\hline Yes $(364 / 305 / 59)$ & $63(20.7)$ & $29(49.2)$ & $<0.001^{\#}$ \\
\hline \multicolumn{4}{|l|}{ Gold-stage $(364 / 305 / 59)$} \\
\hline 1: $\mathrm{FEV}_{1} \geq 80 \%$ & $9(3)$ & $1(1.7)$ & $0.006^{\#}$ \\
\hline $2: 50 \% \leq \mathrm{FEV}_{1}<80 \%$ & $107(35.2)$ & $12(20.0)$ & \\
\hline $3: 30 \% \leq \mathrm{FEV}_{1}<50 \%$ & $138(45.2)$ & $32(54.2)$ & \\
\hline 4: $\mathrm{FEV}_{1}<30 \%$ & $51(16.8)$ & $14(23.3)$ & \\
\hline \multicolumn{4}{|l|}{ Lung function } \\
\hline $\mathrm{FEV}_{1}$ liters $(364 / 305 / 59)$ & $1.08 \pm 0.45$ & $0.86 \pm 0.29$ & $<0.001$ \\
\hline $\mathrm{FEV}_{1}$ pred\% $(364 / 305 / 59)$ & $46.24 \pm 16.93$ & $39.76 \pm 14.75$ & 0.006 \\
\hline $\mathrm{FEV}_{1} / \mathrm{VC}(364 / 305 / 59)$ & $42.36 \pm 11.95$ & $37.71 \pm 10.41$ & 0.003 \\
\hline \multicolumn{4}{|l|}{ Body box } \\
\hline RV/TLC pred\% (227/188/39) & $145.35 \pm 28.74$ & $150.65 \pm 28.12$ & 0.290 \\
\hline \multicolumn{4}{|l|}{ Diffusion } \\
\hline DLCO/SB pred\% $(206 / 179 / 27)$ & $47.90 \pm 17.78$ & $39.26 \pm 16.94$ & 0.017 \\
\hline \multicolumn{4}{|l|}{ Previous admission } \\
\hline Admission ever (364/305/59) & $161(52.8)$ & 43 (72.9) & $0.004^{\#}$ \\
\hline \multicolumn{4}{|l|}{ Admissions last 2 year } \\
\hline An admission (364/305/59) & $127(41.6)$ & $34(57.6)$ & $0.024^{\#}$ \\
\hline-0 & $178(58.6)$ & $25(41.7)$ & $0.081^{\#}$ \\
\hline-1 & $68(22.4)$ & $21(35.0)$ & \\
\hline-2 & $30(9.9)$ & $5(8.3)$ & \\
\hline$-\geq 3$ & $29(9.5)$ & $8(10.2 .0)$ & \\
\hline \multicolumn{4}{|l|}{ An admission } \\
\hline - non-frequent 0-1 & $246(80.9)$ & $46(76.6)$ & $0.635^{\#}$ \\
\hline - frequent & $59(19.3)$ & $13(22.0)$ & \\
\hline Acute NPPV & $34(11.2)$ & $10(16.7)$ & $0.277^{\#}$ \\
\hline \multicolumn{4}{|l|}{ Comorbidities } \\
\hline Charlson,'s index & $2.2 \pm 1.41$ & $2.36 \pm 1.58$ & 0.500 \\
\hline
\end{tabular}


Table 5.3 (continued)

\begin{tabular}{|c|c|c|c|}
\hline Characteristics (n) & $\begin{array}{c}\text { Alive } \\
(n=305)\end{array}$ & $\begin{array}{c}\text { Deceased } \\
(n=59)\end{array}$ & p-value \\
\hline \multicolumn{4}{|l|}{$\mathrm{CCl}$ category } \\
\hline CCI1-2points & 207 (67.9) & $38(64.4)$ & $0.604^{\#}$ \\
\hline $\mathrm{CCl} \geq 3$ points & $98(32.1)$ & $21(35.6)$ & \\
\hline \multicolumn{4}{|l|}{ Home treatment } \\
\hline None $(359 / 298 / 59)$ & $15(5.0)$ & $1(1.7)$ & $0.487^{\#}$ \\
\hline ICS (351/294/57) & $229(82.1)$ & 47 (83.9) & $0.740^{\#}$ \\
\hline SABA $(353 / 295 / 58)$ & $167(59.2)$ & $39(68.4)$ & $0.194^{\#}$ \\
\hline LABA $(352 / 295 / 57)$ & $244(87.1)$ & 47 (83.9) & $0.519^{\#}$ \\
\hline SAMA (350/294/49) & $71(25.4)$ & $22(40.0)$ & $0.028^{\#}$ \\
\hline LAMA (353/295/57) & $220(78.6)$ & 45 (78.9) & $0.950^{\#}$ \\
\hline Prednisolone*(352/295/57) & $26(9.3)$ & $11(19.6)$ & $0.023^{\#}$ \\
\hline Antibiotics* $(351 / 293 / 58)$ & $33(11.3)$ & $6(10.3)$ & $1.000^{\#}$ \\
\hline LTOT (355/296/59) & $67(23.8)$ & $26(44.8)$ & $0.001^{\#}$ \\
\hline CPAP $(352 / 293 / 59)$ & $12(4.1)$ & $1(1.7)$ & $0.704^{\#}$ \\
\hline cNIV $(352 / 293 / 59)$ & $13(4.4)$ & $1(1.7)$ & $0.480^{\#}$ \\
\hline
\end{tabular}

Data presented as $\mathrm{n}(\%)$ or medium with standard deviation depicted after \pm , unless otherwise stated. "Pearson Chi square, *maintenance therapy. Abbreviations: FEV ${ }_{1}$ : forced expiratory volume in 1 second, $\mathrm{FEV}_{1} / \mathrm{FVC}$ : ratio $\mathrm{FEV}_{1} / \mathrm{FVC}, \mathrm{RV} / \mathrm{TLC}$ : ratio residual volume and total lung capacity, TLCO: diffusion capacity of carbon monoxide, NPPV: non-invasive positive pressure ventilation, cNIV: chronic NPPV, CCI: Charlson's comorbidity index, ICS: inhalation corticosteroids, SABA: short acting beta agonist, LABA: long acting beta agonist, SAMA: short acting muscarinic antagonist, LAMA: long acting muscarinic antagonist, LTOT: long term oxygen therapy, CPAP: continuous positive pressure ventilation, pred\%: percentage of predicted value.

Table 5.4 Measurements of patients at emergency room (ER) presentation and hospitalized for ECOPD, stratified by 90 -days outcome.

\begin{tabular}{|c|c|c|c|}
\hline Characteristics (n) & $\begin{array}{c}\text { Alive } \\
(n=305)\end{array}$ & $\begin{array}{c}\text { Deceased } \\
(n=59)\end{array}$ & p-value \\
\hline \multicolumn{4}{|l|}{ Vital signs } \\
\hline Temperature, C (323/275/48) & $37.3 \pm 0.93$ & $37.1 \pm 1.25$ & 0.189 \\
\hline Systolic BP, mmHg(355/298/57) & $140 \pm 25.91$ & $143 \pm 28.9$ & 0.561 \\
\hline Diastolic BP, mmHg (355/298/57) & $76 \pm 16.15$ & $78 \pm 17.02$ & 0.411 \\
\hline Pulse, beats/min $(357 / 300 / 57)$ & $99 \pm 20.52$ & $108 \pm 22.04$ & 0.005 \\
\hline Pulse $>100 / \mathrm{min}$ & $116(32.5)$ & $32(9.0)$ & 0.011 \\
\hline $\mathrm{SpO}_{2} \%$ & $91 \pm 5.38$ & $89 \pm 9.47$ & $0.332^{\#}$ \\
\hline $\mathrm{SpO}_{2}<90 \%$ & $126(42.0)$ & $29(50.0$ & 0.260 \\
\hline Extra $\mathrm{FiO}_{2}(335 / 283 / 52)$ & $164(58.0)$ & $42(80.8)$ & $0.002^{\#}$ \\
\hline \multicolumn{4}{|l|}{ Physical examination } \\
\hline Respiratory rate /min $(173 / 148 / 25)$ & $25 \pm 6.00$ & $26 \pm 6.84$ & 0.313 \\
\hline Confused $(27 / 5 / 3)$ & $5(29.4)$ & $3(30.0)$ & $0.974^{\#}$ \\
\hline Use of accessory muscles (34/23/7) & $23(85.2)$ & $7(100)$ & $0.278^{\#}$ \\
\hline Elevated CVP (119/100/19) & $15(15)$ & $5(26.3)$ & $0.227^{\#}$ \\
\hline Peripheral edema $(307 / 258 / 49)$ & $76(29.5)$ & $12(24.5)$ & $0.481^{\#}$ \\
\hline Wheezing (360/301/59) & $180(59.8)$ & $33(45.2)$ & $0.427^{\#}$ \\
\hline Crackles on inspiration $(360 / 299 / 59)$ & $95(31.6)$ & $20(33.9)$ & $0.725^{\#}$ \\
\hline $\mathrm{BMI}, \mathrm{kg} / \mathrm{m}^{2}(353 / 301 / 52)$ & $25.21 \pm 6.45$ & $23.74 \pm 5.93$ & 0.126 \\
\hline Underweight $\mathrm{BMI}<18.5 \mathrm{~kg} / \mathrm{m}^{2}$ & $39(13.0)$ & $13(25.0)$ & $0.033^{\#}$ \\
\hline Overweight $\mathrm{BMI}>25 \mathrm{~kg} / \mathrm{m}^{2}$ & $132(44)$ & $19(35.8)$ & $0.269^{\#}$ \\
\hline
\end{tabular}


Table 5.4 (continued)

\begin{tabular}{|c|c|c|c|}
\hline Characteristics (n) & $\begin{array}{c}\text { Alive } \\
(n=305)\end{array}$ & $\begin{array}{c}\text { Deceased } \\
(n=59)\end{array}$ & p-value \\
\hline \multicolumn{4}{|l|}{ Arterial blood gas. } \\
\hline $\mathrm{pH}(362 / 303 / 59)$ & $7.38 \pm 0.079$ & $7.33 \pm 0.155$ & $<0.001$ \\
\hline$<7.25$ & $23(7.6)$ & $16(26.7)$ & $<0.001^{\#}$ \\
\hline $7.25-7.35$ & $66(21.9)$ & $14(23.3)$ & \\
\hline$>7.35$ & $214(70.6)$ & $29(49.2)$ & \\
\hline $\mathrm{PaCO}_{2} \mathrm{kPa}(362 / 303 / 59)$ & $6.70 \pm 2.14$ & $8.46 \pm 3.01$ & $<0.001$ \\
\hline $\mathrm{PaCO}_{2} \geq 6.0 \mathrm{kPa}(362 / 303 / 59)$ & $160(52.8)$ & $46(78.0)$ & $<0.001^{\#}$ \\
\hline $\mathrm{PaCO}_{2} \geq 7.0 \mathrm{kPa}(358 / 299 / 59)$ & $1141(37.6)$ & $40(67.8)$ & $0.000^{\#}$ \\
\hline $\mathrm{PaCO}_{2}>9.1 \mathrm{kPa}(362 / 303 / 59)$ & $36(11.9)$ & $25(42.4)$ & $0.000^{\#}$ \\
\hline $\mathrm{HCO}_{3}^{-} \mathrm{mmol} / \mathrm{L}$ & $28.99 \pm 6.10$ & $32.05 \pm 6.80$ & 0.001 \\
\hline $\mathrm{HCO}_{3}{ }^{-} \geq 27.0 \mathrm{mmol} / \mathrm{L}(358 / 299 / 58)$ & $175(58.5)$ & $47(79.7)$ & $0.002^{\#}$ \\
\hline Base excess $(358 / 299 / 59)$ & $2.95 \pm 4.80$ & $4.27 \pm 6.03$ & 0.066 \\
\hline$<-2.5$ & $32(10.7)$ & $8(13.3)$ & $0.010^{\#}$ \\
\hline$-2.5 \mathrm{t} / \mathrm{m} 2.5$ & $129(43.3)$ & $142(39.7)$ & \\
\hline$>2.5$ & $138(46.2)$ & $38(64.4)$ & \\
\hline $\mathrm{PaO}_{2} \mathrm{kPa}(358 / 299 / 59)$ & $7.83 \pm 2.32$ & $9.79 \pm 5.54$ & 0.009 \\
\hline $\mathrm{SaO}_{2} \%$ (in $\mathrm{ABG}$ ) & $85.77 \pm 7.94$ & $87.42 \pm 9.15$ & 0.156 \\
\hline $\mathrm{SaO}_{2}<90 \%(358 / 300 / 58)$ & $126(42.0)$ & $29(50.0)$ & $0.260^{\#}$ \\
\hline Extra $\mathrm{FiO}_{2}(314 / 266 / 48)$ & $102(38.2)$ & $40(85.1)$ & $<0.001^{\#}$ \\
\hline $\mathrm{PaO}_{2}<7.5 \mathrm{kPa}$ & $156(52.2)$ & $22(37.3)$ & $0.037^{\#}$ \\
\hline \multicolumn{4}{|l|}{ Laboratory results } \\
\hline Hemoglobin mmol/L (354/296/58) & $8.47 \pm 1.19$ & $7.89 \pm 1.22$ & 0.001 \\
\hline Low $<8.2$ & $116(39.3)$ & $35(59.3)$ & $0.010^{\#}$ \\
\hline Normal $8.2-11.0$ & $177(59.8)$ & $23(39.7)$ & \\
\hline High $>11.0$ & $3(1.0)$ & $0(0.0)$ & \\
\hline Hematocrit (188/158/30) & $0.42 \pm 0.06$ & $0.41 \pm 0.06$ & 0.124 \\
\hline WBC $10^{9} / \mathrm{L}(362 / 303 / 59)$ & $13.17 \pm 5.75$ & $14.58 \pm 6.98$ & 0.099 \\
\hline$W B C>1110^{9} / L$ & $178(58.7)$ & $40(67.8)$ & $0.194^{\#}$ \\
\hline Eosinophilia \% (138/112/26) & $1.19 \pm 2.15$ & $0.38 \pm 0.64$ & 0.001 \\
\hline Na mmol/L (360/301/59) & $137.21 \pm 4.82$ & $137.59 \pm 5.16$ & 0.577 \\
\hline $\mathrm{Na}<135 \mathrm{mmol} / \mathrm{L}$ & $71(23.6)$ & $16(27.1)$ & $0.562^{\#}$ \\
\hline Urea mmol/L (347/288/59) & $7.10 \pm 4.33$ & $10.80 \pm 6.84$ & 0.000 \\
\hline Urea $\geq 8 \mathrm{mmol} / \mathrm{L}$ & $79(27.3)$ & $35(60.3)$ & $<0.001^{\#}$ \\
\hline Creatinine mmol/L (357/298/59) & $91.93 \pm 60.45$ & $103.78 \pm 74.16$ & 0.187 \\
\hline Creatinine $>115 \mu \mathrm{mol} / \mathrm{L}$ & $57(19.1)$ & $16(27.1)$ & $0.164^{\#}$ \\
\hline ASAT U/L (159/126/33) & $71.27 \pm 383.01$ & $29.09 \pm 19.49$ & 0.529 \\
\hline ASAT $>35 \mathrm{U} / \mathrm{L}$ & $41(32.5)$ & $8(24.2)$ & $0.358^{\#}$ \\
\hline ALAT U/L (157/127/30) & $47.76 \pm 174.23$ & $24.67 \pm 18.20$ & 0.471 \\
\hline ALAT $>45 \mathrm{U} / \mathrm{L}$ & $19(15.0)$ & $1(3.3)$ & $0.126^{\#}$ \\
\hline LDH U/L (118/96/22 & $317.93 \pm 353.93$ & $279.86 \pm 104.37$ & 0.619 \\
\hline $\mathrm{LDH} \geq 500 \mathrm{U} / \mathrm{L})$ & $7(7.3)$ & $1(4.5)$ & $1.00^{\#}$ \\
\hline Troponin $>14 \mathrm{ng} / \mathrm{L}$ & $77(25.2)$ & $12(20.3)$ & $0.422^{\#}$ \\
\hline NT-proBNP pmol/L (139/115/24) & $393.09 \pm 617.85$ & $639.30 \pm 697.83$ & 0.085 \\
\hline NT-proBNP $\geq 500 \mathrm{pmo} / \mathrm{L}$ & $20(17.4)$ & $11(45.8)$ & $0.002^{\#}$ \\
\hline Albumin g/l & $31.98 \pm 4.29$ & - & \\
\hline Albumin <35 g/l (9/8/1) & $6(75)$ & $1(11.1)$ & $0.333^{\#}$ \\
\hline CRP, mg/L (361/330/31) & $68.41 \pm 86.16$ & $84.01 \pm 92.88$ & 0.210 \\
\hline$<50$ & $183(60.6)$ & $29(49.2)$ & \\
\hline $50-200$ & $90(29.9)$ & $21(35.0)$ & \\
\hline$>200$ & $29(9.6)$ & $9(15.0)$ & $0.208^{\#}$ \\
\hline
\end{tabular}


Table 5.4 (continued)

\begin{tabular}{lccc}
\hline Characteristics (n) & $\begin{array}{c}\text { Alive } \\
(\mathbf{n = 3 0 5 )}\end{array}$ & $\begin{array}{c}\text { Deceased } \\
(\mathbf{n = 5 9 )}\end{array}$ & p-value \\
\hline Glucose mmol/L (321/267/54) & $7.75 \pm 2.85$ & $8.44 \pm 3.42$ & 0.115 \\
Glucose $\geq 11 \mathrm{mmol} / \mathrm{L}$ & $34(12.7)$ & $8(14.8)$ & $0.679^{\#}$ \\
Chest X-ray (363/304/59) & & & \\
$\quad$ Consolidation & $82(27.0)$ & $25(42.4)$ & $0.018^{\#}$ \\
Signs of congestion & $44(14.5)$ & $8(13.6)$ & $0.854^{\#}$ \\
ECG (279/238/41) & & & \\
Atrial fibrillation & $24(10.1)$ & $14(34.1)$ & $<0.001^{\#}$ \\
Signs of ischemia & $19(6.9)$ & $3(5.6)$ & $0.716^{\#}$ \\
\hline
\end{tabular}

Data presented as $\mathrm{n}(\%)$ or medium with standard deviation depicted after \pm , unless otherwise stated. \#(Pearson) Chi square. Abbreviations: $\mathrm{BP}$ : blood pressure, $\mathrm{SpO}_{2}$ : oxygen saturation measured by pulse oximetry, $\mathrm{FiO}_{2}$ : fraction of inspired oxygen, CVP: central venous pressure, $\mathrm{BMI}$ : body mass index, $\mathrm{SaO}_{2}$ : oxygen saturation measured in arterial blood gas, WBC: white blood cell, Na: sodium, ASAT: aminotransferase, ALAT: alanineaminotranferase, LDH: lactic acid dehydrogenase, NT proBNP: brain natriuremic peptide, CRP: Creactive protein, ECG: electrocardiogram, ABG: arterial blood gas, ER: emergency room, kPa: kilopascal, $\mathrm{mmol} / \mathrm{l}$ : millimol per liter, $\mu \mathrm{mol} / \mathrm{L}$ : micromol per liter, pmol/L: picomol per liter, U/L: units per liter, $\mathrm{ng} / \mathrm{L}$ : nanogram per liter, \%: percentage.

Figure 5.2 Visual representation of the classification tree. The classification and regression tree (CART) analysis in patients with hospitalized for ECOPD $(n=306)$. The decision tree is built from full data from 306 patients. The tree first splits patients based on $\mathrm{PaCO}_{2}$ levels. Those with lower $\mathrm{PaCO}_{2}$ are then split on age, and younger ( $<80$ years) patients are again split on BMI. After this step patients were split again on prior admission in last 2 years. Patients are thus placed into one of five category, where the number of the patient is given in the category and the percentage of 90 day mortality is depicted as \%. The $p$ value describes the statistical significance of the node.

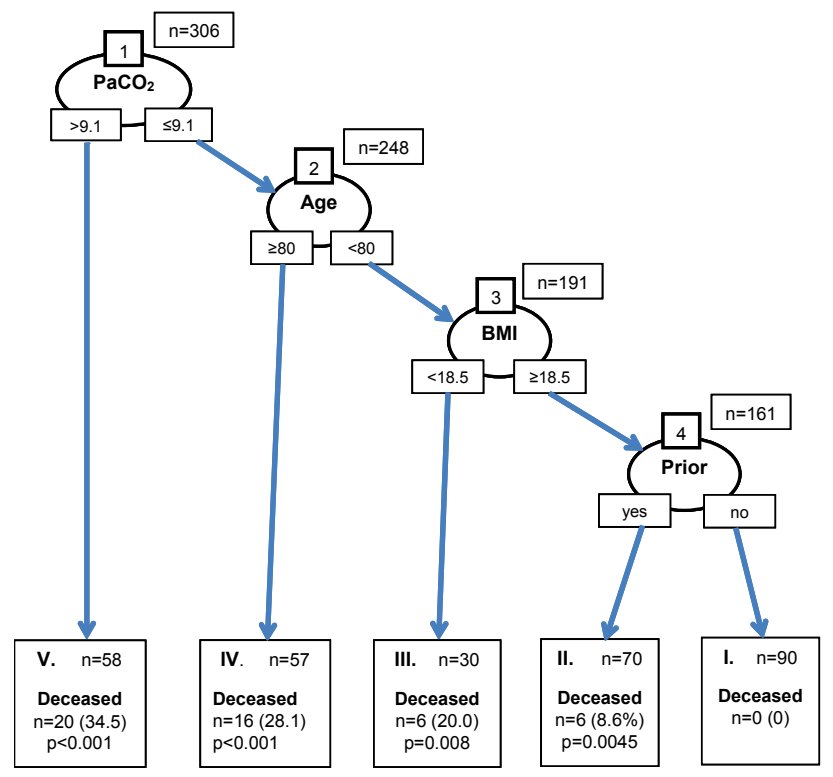

Abbreviations: $\mathrm{BMI}$ : Body mass index, $\mathrm{PaCO}_{2}$ in $\mathrm{kPa}$, Age in years, $\mathrm{BMI}$ in $\mathrm{kg} / \mathrm{m}^{2}$, prior means prior admission in last two years. 


\section{Discussion}

The present study confirmed the high in-hospital and short-term mortality rates associated with severe ECOPD; more than $8 \%$ of patients died during hospitalization and more than one in six did not survive after 90 days. Using a very extensive set of demographic, clinical, biochemical and other parameters collected at hospital admission, this study identified four independent predictors of 90-days mortality: age, $\mathrm{PaCO}_{2}$, urea and BMI. In a subsequent CART analysis, the four most discriminant predictive factors for 90-days mortality were: $\mathrm{PaCO}_{2}>9.1 \mathrm{kPa}$, Age $\geq 80 \mathrm{y}, \mathrm{BMI}$ $<18.5 \mathrm{~kg} / \mathrm{m}^{2}$, and prior admission for ECOPD during the previous 2 years. This CART enables risk stratification of COPD patients with severe ECOPD and thus may support clinical decision making.

Previous studies showed an in-hospital mortality of $4.8-10.4 \%^{7-9,24}$ and a 90 -days mortality after admission of $9.0-13.9 \% .^{8,9,12,18,25}$ Thus, the present study reported a comparable in-hospital mortality. However, 90-day mortality in the present cohort was slightly worse (16.2\%). Kumar et all reported a 90-day mortality of $9 \%$. Nineteen percent of that cohort used LTOT, which is less compared to ours. ${ }^{25}$ Data in this study about the severity of the exacerbation were not available. ${ }^{25}$ Another study described by Roberts et al reported 90-day mortality of $13,9 \%$ with a mean $\mathrm{PaCO}_{2} 5.9 \mathrm{kPa}$ and mean

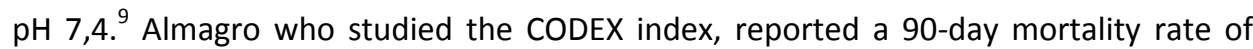
$4.8 \% .{ }^{12}$ In this cohort on admission the mean $\mathrm{PaCO}_{2}$ was $6.3 \mathrm{kPa} .{ }^{12}$ In our cohort, the mean $\mathrm{PaCO}_{2}$ on admission was $7.0 \mathrm{kPa}$. In the cohort of the PEARL study, less patients had LTOT and the mean pH at admission was lower than 7.35 and less patients needed NIV during admission compared to the current study. ${ }^{8}$ Compared with the study cohort of Steer et al. ${ }^{8}$ the current patients had a lower $\mathrm{pH}$ and a higher $\mathrm{PaCO}_{2}$ and a larger percentage of patients (30\%) needed NIV. ${ }^{18}$ This suggests, that the patients in our cohort had a more pronounced respiratory failure which might have a negative impact on the short term survival.

The 90-day mortality was increased in patients requiring mechanical ventilation $(27.8 \%)$ compared to patients not requiring mechanical ventilation (10.8\%) (Pearson- $\mathrm{x}^{2}: 16.706$; $p<0.0001)$. This is comparable to the study by Roberts et al. ${ }^{26}$ who observed a 90 -day mortality of $33 \%$ in those receiving non-invasive ventilation and $11 \%$ in those hospitalized COPD patients not receiving NIV. Almagro and colleagues did not take mechanical ventilation into account in their study on determinants of 90-day mortality in patients hospitalized for COPD exacerbation. ${ }^{12}$ These combined results suggest that indeed patients treated in usual clinical care with mechanical ventilation have poor 
outcomes, potentially as this intervention is the last resource of medical care for these very severe patients.

For hospitalized ECOPD several predictors ${ }^{7,8,11,12,19,27-30}$ for short-term but mainly for long-term mortality have been identified. Several multi-dimensional indexes have been proposed for predicting in-hospital mortality or mortality after hospital discharge. $8,10,12,18,19,31$ In 2010 the CURB65 score was suggested to be effective for predicting early mortality in hospitalized ECOPD. ${ }^{16}$ However, Steer et al showed that the use of the CURB65 score was suboptimal in these patients. ${ }^{32}$ The DECAF score predicts only the in-hospital mortality. ${ }^{8}$ A predictive score was presented by Quintana in 2014 for 30-day mortality in patient with ECOPD attending in emergency department whether admitted or discharged at home. ${ }^{19}$ The LACE score is not disease specific and not primarily designed to predict mortality but predicts readmissions rate. $^{17}$ In the PEARL score and the CODEX score readmission or death after admission for ECOPD was used as a combined primary endpoint after 90 days. ${ }^{12,18}$ But to our knowledge none of these predictive scores have been validated for 90-day mortality assessment after hospital admission or were not feasible in ER setting.

In the current study, high level of $\mathrm{PaCO}_{2}(>9.1 \mathrm{kPa})$ was assessed as the first discriminant predictive factor in the stratification tree. This finding is in line with previous studies reporting a relationship between high levels of $\mathrm{PaCO}_{2}$ on admission and survival in ECOPD. ${ }^{6,7,33}$ However, none of the previous predictive scores included $\mathrm{PaCO}_{2}$ levels. Instead, the DECAF score included acidaemia as a surrogate. In most studies, dyspnoea or use of accessory inspiratory muscles were predictors for mortality. These parameters indicate respiratory distress or reflect respiratory failure. Alternatively, high levels of $\mathrm{PaCO}_{2}$ reflect decreased alveolar ventilation, indicating very severe respiratory pump failure in which patients in acute settings are dyspnoeic or use their inspiratory accessory muscles. Acidaemia and high levels of $\mathrm{PaCO}_{2}$ can be considered as an potentially modifiable factors in acute setting. ${ }^{30}$

The present study observed that elderly patients with severe ECOPD had worse outcomes, which is in concordance with previous studies. ${ }^{6}$ In addition, a review concluded that age was consistently associated with short term mortality in hospitalized COPD adults. ${ }^{30}$ Age is a physiological parameter used in many disease indices and clinical scales such as the APACHE ${ }^{34,35}$ and LACE $^{17,36}$ score. Multi-morbidity, progressive muscle atrophy and lung function loss, declining biological cell and system dysfunction are among the factors underlying the increased mortality risk with aging. ${ }^{15}$ As in other studies ${ }^{18,19}$, the cut-off for age was rather high, around 80 years. 
The third factor in the present risk stratification model was BMI. Low body weight is a well-recognized determinant of poor survival in stable COPD ${ }^{37}$ and is incorporated in the BODE index. ${ }^{13}$ The impact of $\mathrm{BMI}$ on survival in unstable COPD is less studied. Lainscak et al observed highest long-term mortality rates in hospitalized patients with $\mathrm{BMI}<21.55 \mathrm{~kg} / \mathrm{m}^{2}{ }^{38}$ As weight gain is associated with reduced mortality ${ }^{39}$, body weight is an important treatable trait in COPD.

Finally, previous hospitalization for ECOPD within 2 years was found to be the last discriminating factor in the CART model. In the CODEX model and in the PEARL score, previous admissions within 1 year was also identified as an important predictor. In other previous studies investigating predictors of mortality after hospitalized ECOPD, a previous admission was found to be an important contributor to mortality. ${ }^{40}$ These results highlight the great clinical importance of prevention of hospitalized ECOPD in the management of COPD and justifies that hospitalizations for ECOPD are taken into account for the risk stratification in the GOLD guideline. ${ }^{22}$

Another strong independent predictor we found in our data was circulating urea. ${ }^{10,32}$ This finding is in line with our report about urea as an independent predictor for 1-year mortality. ${ }^{7}$ Interestingly, urea is also used as a factor in the CURB65 score. Impairment of the renal function and relationship with high levels of $\mathrm{PaCO}_{2}$ was already suggested in a former review. ${ }^{41}$

Other strong predictors we found were infiltration ${ }^{8,32,42}$, Gold stage ${ }^{43,44}$, supported living $^{45}$, admission in the last two year ${ }^{27,46}$ and LTOT. $^{19}$

It is well-known that comorbidities contribute to mortality in hospitalized COPD patients and indeed the CODEX (comorbidity, obstruction, dyspnea, and previous severe exacerbations) index can accurately predict mortality and hospital readmissions after discharge. ${ }^{12}$ In our study, Charlson comorbidity index was used to classify comorbidities and no difference in average score or percentage of patients with high comorbidity score was observed. This discrepancy may have been caused by the methodology of comorbidity assessment (chart-based versus objectively identified) ${ }^{47}$, the differential impact of comorbidities in COPD patients with different severity of disease $^{48}$ or the limited size of the study population. However, we did observe increased in-hospital and 90-days mortality in COPD patients presenting with comorbid atrial fibrillation (Tables 5.2 and 5.4).

In the current study CART analysis was used in order to develop a prediction model for short-term mortality. CART analysis is a novel, powerful, non-parametric machine 
learning approach that is well suited for variable selection among many candidate predictor variables where these are far from normally distributed and have complex interactions. $^{49}$ This approach is considered complementary to logistic regression techniques. ${ }^{50}$ Another strength of this study was the extensive clinical, biological and biochemical characterisation of the study population, enabling the inclusion of many candidate variables in the prediction model. Also, the dataset used was very complete with few missing patients and no loss to follow up.

Our study had some limitations that need to be addressed. First, the retrospective study design could lead to an information bias. The present study is a single center study which could mean that the results may be less representative for other hospitals. Another important limitation of this retrospective study is the lack of a dyspnoea score,

since several studies showed this outcome is strongly correlated with mortality. ${ }^{8,12,19,32}$ It wasn't possible to include this data into the present analyses because a dyspnoea measure was not routinely recorded in the ER files. On the other hand the study results are based on real life data set and patients were consecutively enrolled in the study without strict in-and exclusion criteria. Another subject to discuss is the effect of policy restrictions on mortality. In our study population 93 (26\%) patients had some sort of policy restrictions before admission. It is possible that these, especially the no mechanical ventilation order contributed to an increased in-hospital mortality. We found in our cohort no statistical difference between the groups, however this could be attributed to the small numbers.

\section{Conclusions}

In terms of clinical implications, the present study identified $\mathrm{pCO}_{2}>9.1 \mathrm{kPa}$, age $\geq 80 \mathrm{y}$, BMI $<18.5 \mathrm{~kg} / \mathrm{m}^{2}$ and prior admission for ECOPD in last two years as independent predictors of short-term mortality in patients hospitalized for ECOPD. The presence of 4 positive factors was associated with $50 \%$ mortality risk, while all patients with absence of all of these factors survived beyond 90 days. This risk stratification tool can give clinicians the opportunity to assess the risk of adverse outcome and to take this in account in shared decision making and the advance care planning leading to personalized adjusted medicine. Obviously, the present results need to be validated in a subsequent prospective study. 


\section{Appendix S5.1-Methods (extended version)}

\section{Design and population}

We conducted a retrospective, observational cohort study at the department of respiratory medicine of Maastricht University Medical Center (MUMC) in Maastricht, the Netherlands. Clinical data of eligible patients who had been hospitalized for ECOPD on our pulmonary ward between 01-06-2011 and 31-12-2014 were included in the current analyses. Patients were initially identified on the basis of the ICD9(International Statistical Classification of Diseases and Related Health Problems) and ICD-10 (2014) codes for COPD (J42-44) and 'acute respiratory symptoms or pneumonia' (ICD-9 786.0-9 and ICD-10 J12-16) in the hospital electronic database. The index admission was the last admission during the study period. The inclusion criteria were: 1) ECOPD defined as sudden increase in one or more of the following; dyspnoea, cough or sputum production and treatment with systemic glucocorticoids and/or antibiotics and 2) confirmation of obstructive lung function by a ratio of post-bronchodilator forced expiratory volume in 1 second to forced vital capacity (FEV1/FVC) $<70 \%{ }^{22,51}$ in the medical record of the patient. Exclusion criteria were: age $<40$ years, active pulmonary malignancy based on medical records, follow-up in another hospital and primary reason for hospitalization other than ECOPD. Patients admitted for ECOPD and consolidation on chest X-ray were not excluded. This study was conducted according to the Declaration of Helsinki (59 ${ }^{\text {nd }}$ WMA General Assembly, Seoul, October 2008) and Good Clinical Practice guidelines. According the Dutch law, for this retrospective study informed consent was not required. Because we used de-identified data in this retrospective study ethical approval was not necessary according the local ethical committee of the MUMC (METC 13-4-041).

\section{Data collection}

Anonymized demographic, clinical and laboratory parameters were extracted from the electronic records of the patients. Age, gender, smoking history, medical history including comorbidities, previous admissions for ECOPD, post-bronchodilator forced expiratory volume in 1 second $\left(\mathrm{FEV}_{1}\right)$, forced vital capacity (FVC), FEV 1 /FVC ratio, GOLD 2014 stage for airflow limitation, residual volume (RV), total lung capacity (TLC), diffusion capacity (DLCO) (recorded lung function maximal 3 year prior the index admission), need for home care, living status, respiratory medications and use of long term oxygen treatment (LTOT) were collected at time of the index admission. The following parameters from assessment at during presentation at the ER were collected: vital signs, physical examination, body mass index (BMI), arterial blood gases (ABG), 
blood chemistry, electrocardiogram (rhythm and signs of ischemia), chest X-ray (consolidation and signs of congestions). Treatment at ER and the need for acute mechanical ventilation, according to our hospital protocol, at ER or in the first 24 hours was recorded. Comorbidities were quantified using previously validated Charlson-index $(\mathrm{CCl})^{44}$ and classified according to severity as none $(\mathrm{CCl}$ score $=0)$, medium $(\mathrm{CCl}$ score $=1-2$ ) or high ( $\mathrm{CCl}$ score $=\geq 3$ )..$^{52}$ Additionally, other comorbidities that were considered relevant were also recorded.

\section{Outcomes and follow-up}

In-hospital and 90-day all-cause mortality rates were determined for each patient by review of clinical notes and electronic patient records; survival status and survival time were recorded. The primary outcome was 90-day mortality. In case of an in-hospital death the date was recorded and verified. The date of death of patients who died after hospital discharge was verified from hospital records, general practitioner's records or death records of the patients' residence authority. Survival status of all patients was confirmed in the same way.

\section{Statistical analysis}

All statistical analyses were carried out using SPSS for MAC version 23.0 and $\mathrm{R}$ software environment (version 3.1.0). A descriptive analysis of the patients included into this study was performed and the demographic variables of the patients who deceased or not, were computed. Distribution of data was assessed by Kolmogorov-Smirnov test and normally distributed data are presented as mean and standard deviation whereas non-parametric data are presented as median and first and third quartiles (25-75\% percentiles). Categorical data are presented as percentages. After these descriptive analyses, univariate analyses were performed using independent-tests, $\mathrm{x}^{2}$.tests or fisher's exact tests as appropriate to compare the characteristics of patients who survived the hospital admission and 90 days or who died during hospital stay and within 90-days of follow-up. Continuous variables that were not normally distributed were categorized and divided into clinically relevant groups prior to analyses.

Clinically relevant factors with a p-value $<0.05$ in univariate analysis were included in the multivariate analysis. Data was restricted to patients with all candidate covariates available. A baseline model was made by logistic regression using a model which included all candidate covariates. A search was then made over all possible combinations of these covariates, again using a logistic regression formulation, and using the Akaike information criteria (AIC) to determine model fit. 
These same clinically relevant and nominally significant predictors were used as input to the CART model. ${ }^{23}$ CART models divide subjects into relevant classes based on categorical divisions of predictor variables. The procedure which fits the tree to the data automatically selects the most relevant predictor variables and also the value for each variable which provides the most discriminant power. Since this tree is based on data with a strong bias towards survivors, a randomized repeated subsampling method was implemented to evaluate the importance of selected predictors. Each iteration of the resampling randomly selected 34 of the 43 deceased (approximately $80 \%$ ) and 51 of the 184 survivors (28\%), yielding a subsample with a 1:1.5 deceased to survivor ratio. A CART was fit to this subset, and both the variables selected and their cut-points were recorded. The random subsampling was repeated 100 times, and the frequency of selection of each of the predictor variables, as well as the mean value of the chosen cut-point were recorded. 


\section{Appendix S5.2-Results (extended version)}

\section{Subjects}

The flow chart for patient selection is shown in figure 1. In total 4868 admissions with the indications 'acute respiratory symptoms, COPD or pneumonia' were screened between June 2011 and December 2014. The primary reason for exclusion was the absence of ECOPD diagnosis at admission. Finally, 364 patients who had been admitted with to the pulmonary ward in MUMC with a primary diagnosis of ECOPD and treated with systemic corticosteroids and/or antibiotics were eligible for analysis.

\section{Characteristics of the study population}

Table 5.1 shows the clinical characteristics of the study population. On average, these were elderly male and female COPD patients with moderate to severe airflow limitation and significantly impaired diffusion capacity. The majority of patients was admitted from their home situation and had not been hospitalized in the two previous years. Patients had multiple comorbidities and more than one quarter of them was using LTOT. Vital signs and laboratory results at the time of presentation at the ER are presented in Table 5.2. Patients were hemodynamically stable with increased breathing rate and clinical signs of airflow limitation. One third of them presented with respiratory acidosis while the majority received acute oxygen therapy. On x-ray, one third of patients had signs of pneumonia.

\section{Follow-up and mortality}

During the 90 days follow-up 59 (16.2\%) patients died of which 30 (8.2\%) died inhospital and $38(10.4 \%)$ patients were dead at 30 days after admission. The mean length of hospital stay was $9.0 \pm 7.6$ days. In 115 (31.5\%) patients, mechanical ventilation was needed during the admission; 110 (30\%) patients needed non-invasive ventilation and 15 (4.1\%) patients were treated with invasive ventilation. In 7 patients, noninvasive ventilation (NIV) failed and these were switched to invasive ventilation. Of the patients who required mechanical ventilation $(n=115)$, thirty-two $(27.8 \%)$ died during 90-days follow-up and this group had a significantly increased 90-day mortality rate compared to patients not requiring mechanical ventilation (Pearson- $x^{2}: 16.706$; $p<0.0001)$. The in-hospital mortality in patients using NIV $(n=110)$ was $21(19 \%)$ patients and was significant higher than in patients not using mechanical ventilation (Pearson- $x^{2}$ : $24.535, p<0001)$. Twenty-six patients $(7.8 \%$ of discharged population), had an early 
readmission for respiratory problems within 90-days follow-up. Of these patients 7 died after their readmission.

\section{Univariate analysis}

Patients who died in hospital were older, more care-dependent, had more severe lung function impairment, more often a restricted policy towards cardiopulmonary resuscitation and more often invasive mechanical ventilation (Table 5.1). Previous admission in the last two years was present more often in the deceased patients. Also, LTOT usage was significantly more often present. The number of comorbidities and use of respiratory medications was not different between surviving and non-surviving patients. On presentation at ER, non-survivors were characterized by increased pulse rate, increased need for supplemental oxygen, higher levels of $\mathrm{PaCO}_{2}$ retention, higher level of urea, lower eosinophil counts, increased frequency of consolidation and signs of cardiovascular instability with atrial fibrillation. The same outcomes were observed in patients that died within 90 days (Tables 5.1-5.4).

\section{Multivariate analysis}

The univariate analysis in the dataset suggested 9 clinically relevant and nominally significant candidate coefficients: age (continuous variable), BMI (categorical, $<18.5 \mathrm{~kg} / \mathrm{m}^{2}$ ), infiltration (categorical), previous admission (categorical), LTOT (categorical), supported living (categorical "no extra care", "extra care"), $\mathrm{PaCO}_{2}$ (continuous), Gold stage for airflow limitation (categorical), Urea (continuous). The associated odds ratios and $p$-values for all covariates are shown in Table 5.5. After fitting this model to the 306 patients for whom complete data were available, age, $\mathrm{PaCO}_{2}$ and urea had $\mathrm{p}$-values less than 0.01 (Table S5.1). AIC of the model was 218.6. Analysis of the model's deviance suggested it explained $25 \%$ of the variance in outcomes in this patient sample. The search over all possible models found that the model with the lowest (best) AIC contained the following covariates: age, $\mathrm{BMI}, \mathrm{PaCO}_{2}$, urea. All except BMI ( $p=0.014)$ showed $p$-values $<0.01$. Analysis of the model's deviance suggested it explained $23 \%$ of the variance in outcomes in this patient sample. However, it does so with far fewer parameters than the full model, as reflected in its AIC score of 213.6.

\section{Classification tree analysis}

CART analysis using the full data selected four variables as having discriminant power: $\mathrm{PaCO}_{2}$, age, $\mathrm{BMI}$ and prior admission last two years. These discriminant variables and 
cut-off points were supported by the randomized subsampling. The $\mathrm{PaCO}_{2}$ level was used in $81 \%$ (mean 9.23, standard deviation 0.89 ) of the subsampled trees, age in $64 \%$ $(80.0 \pm 2.2)$ and $\mathrm{BMI}$ in $21 \%$. The cut-off points for $\mathrm{PaCO}_{2}$ level $(9.1 \mathrm{kPa})$ and age (80 years) was assessed by using the CART analysis in multiple subsets. The remaining input variables selected during the univariate analysis were only rarely used.

The decision tree was built from full data from 306 patients. The tree first splits patients based on $\mathrm{PaCO}_{2}$ levels. Those with lower $\mathrm{PaCO}_{2}$ are then split on age, and younger patients are again split on BMI and last split on prior admissions (last two years, see Figure 5.2). Patients were thus placed into one of five categories. Patients are initially divided based on $\mathrm{PaCO}_{2}$ level of $9.1 \mathrm{kPa}$. Of the 58 patients with $\mathrm{PaCO}_{2}$ higher than this threshold, $34.5 \%$ ( $n=20$ ) had 90-day mortality. For the remaining patients, i.e. those with $\mathrm{PaCO}_{2}$ equal to or less than $9.1 \mathrm{kPa}$, the next most discriminant factor was age greater than 80 years old $(n=57)$. In this category $16(28.1 \%)$ patients were dead after 90-days follow-up. For the remaining patients, i.e. those with $\mathrm{PaCO}_{2}$ equal to or less than $9.1 \mathrm{kPa}$ and age less than 80 years, the most discriminant factor was $\mathrm{BMI}$ $<18.5 \mathrm{~kg} / \mathrm{m}^{2}$. Of the 30 patients in this category, $20 \%(\mathrm{n}=6)$ died within 90 days. Prior admissions in last two years was the next most discriminating factor. Of the 70 patients in group II 8.6\% $(n=6)$ died within 90 days. Of the remaining 90 patients $\left(\mathrm{PaCO}_{2}\right.$ less than $9.1 \mathrm{kPa}$, aged under 80 years, BMI $>18.5 \mathrm{~kg} / \mathrm{m}^{2}$ and no prior admission), no one died within 90 days (Figure 5.2 and Table S5.2). The remaining variables were not used by the tree. Figure 5.2 gives a visual representation of the tree. In the CART model the most predictive factors are level of $\mathrm{PaCO}_{2}, \mathrm{Age}, \mathrm{BMI}$ and prior admissions for ECOPD in last two years.

\section{Effect of policy restrictions on mortality}

Another result to discuss is the effect of policy restrictions on mortality. In our study population $93(26 \%)$ patients had some sort of policy restrictions before admission, of which the vast majority 91 (98\%) had a do not resuscitate (DNR) order, 80 (86\%) a no mechanical ventilation order and $10(11 \%)$ a no NIV order. It is possible that these, especially the no mechanical ventilation order contributed to an increased in-hospital mortality. We found in our cohort no statistical difference between the groups, however this could be attributed to the small numbers. In addition, the effect of policy restrictions is smaller due to decisions of medical personnel to withhold further treatment in case of critically ill patients because it was deemed useless except in the nature of comfort or palliative care. This could result in policy restrictions on medical grounds in the group without policy restrictions at ER presentation. In a total of 
32 patients the policy restrictions were altered in the ER, in some cases on the request of the patient and in some cases on medical grounds.

Table S5.1 Multivariate model using all 9 coefficients to predict 90 days mortality after hospitalized ECOPD at admission analysed in patients

\begin{tabular}{lcccc}
\hline & OR & Beta & 95\% Cl & p-value \\
\hline Age $^{*}$ (years) & 1.09 & 0.0857 & $0.0605-0.111$ & 0.000669 \\
BMI $<18.5 \mathrm{~kg} / \mathrm{m}^{2}$ & 2.72 & 1.0006 & $0.5279-1.473$ & 0.03428 \\
Consolidation on Chest x-ray & 1.74 & 0.5519 & $0.1614-0.924$ & 0.157609 \\
Previous admission last 2 year & 1.29 & 0.2539 & $-0.1446-0.652$ & 0.524056 \\
LTOT & 1.02 & 0.0199 & $-0.4001-0.440$ & 0.962234 \\
Supported living & 1.64 & 0.4974 & $0.0694-0.926$ & 0.245223 \\
PaCO $^{*}(\mathrm{kPa})$ & 1.31 & 0.2732 & $0.1995-0.347$ & 0.000207 \\
Gold stage (according FEV $\left._{1}\right)$ & 1.21 & 0.1932 & $-0.1181-0.504$ & 0.534863 \\
Urea* $^{*}(\mathrm{mmol} / \mathrm{l})$ & 1.14 & 0.1312 & $0.0962-0.166$ & 0.000177 \\
\hline
\end{tabular}

*Continues variable. Shown is the predicted odds ratio (OR) based on the regression coefficient Beta, the $95 \%$ confidence interval for Beta, and the uncorrected p-value. Abbreviations: LTOT: BMI: body mass index, long term oxygen therapy, $\mathrm{FEV}_{1}$ : forced expiratory volume in 1 second. $\mathrm{kPa}$ : kilopascal, $\mathrm{mmol} / \mathrm{l}$ : millimol per liter

Table S5.2 90 days mortality according CART anaylsis in patients with hospitalized for ECOPD.

\begin{tabular}{lcccc}
\hline & $\begin{array}{c}\text { Total } \\
(\mathbf{n = 3 0 6 )}\end{array}$ & $\begin{array}{c}\text { Deceased } \\
(\mathbf{n = 4 8 )}\end{array}$ & $\begin{array}{c}\text { Survivors } \\
(\mathbf{n = 2 5 8 )}\end{array}$ & $\begin{array}{c}\text { \%deceased } \\
(\mathbf{1 5 . 7 \% )}\end{array}$ \\
\hline $\mathrm{PaCO}{ }_{2} \geq 9.1 \mathrm{kPa}$ & 58 & 20 & 38 & 34.5 \\
$\mathrm{Age} \geq 80 \mathrm{y}$ & 57 & 16 & 41 & 28.1 \\
$\mathrm{BMI}<18.5 \mathrm{~kg} / \mathrm{m}^{2}$ & 30 & 6 & 24 & 20 \\
$\mathrm{No} \mathrm{CAB}$ & 161 & 6 & 155 & 3.7 \\
Prior admission & 70 & 6 & 64 & 8.6 \\
No prior admission & 91 & 0 & 91 & 0 \\
Age $\leq 65 y(Y)$ & 85 & 5 & 81 & 5.8 \\
Yprior admission & 33 & 4 & 29 & 12.1 \\
Yno prior admission & 53 & 1 & 52 & 1.9 \\
\hline
\end{tabular}

Abbreviations: $\mathrm{BMI}$ : body mass index, no Cab means: $\mathrm{pCO}_{2}<9.1 \mathrm{kPa}$ and $\mathrm{Age}<80$ y and $\mathrm{BMI}>18.5 \mathrm{~kg} / \mathrm{m}^{2}$; Group $Y$ is Age $\leq 65$ y; Yprior admission: prior admission in last two years. 


\section{References}

1. Dransfield, M.T., et al., Acute Exacerbations and Lung Function Loss in Smokers with and without Chronic Obstructive Pulmonary Disease. Am J Respir Crit Care Med, 2017. 195(3): p. 324-330.

2. Seemungal, T.A., et al., Effect of exacerbation on quality of life in patients with chronic obstructive pulmonary disease. Am J Respir Crit Care Med, 1998. 157(5 Pt 1): p. 1418-22.

3. Waschki, B., et al., Physical activity monitoring in COPD: compliance and associations with clinical characteristics in a multicenter study. Respir Med, 2012. 106(4): p. 522-30.

4. Foo, J., et al., Continuing to Confront COPD International Patient Survey: Economic Impact of COPD in 12 Countries. PLoS One, 2016. 11(4): p. e0152618.

5. Hartl, S., et al., Risk of death and readmission of hospital-admitted COPD exacerbations: European COPD Audit. Eur Respir J, 2016. 47(1): p. 113-21.

6. Groenewegen, K.H., A.M. Schols, and E.F. Wouters, Mortality and mortality-related factors after hospitalization for acute exacerbation of COPD. Chest, 2003. 124(2): p. 459-67.

7. Slenter, R.H., et al., Predictors of 1-year mortality at hospital admission for acute exacerbations of chronic obstructive pulmonary disease. Respiration, 2013. 85(1): p. 15-26.

8. Steer, J., J. Gibson, and S.C. Bourke, The DECAF Score: predicting hospital mortality in exacerbations of chronic obstructive pulmonary disease. Thorax, 2012. 67(11): p. 970-6.

9. Roberts, C.M., et al., Co-morbidities and 90-day outcomes in hospitalized COPD exacerbations. COPD, 2011. 8(5): p. 354-61.

10. Shorr, A.F., et al., Validation of a novel risk score for severity of illness in acute exacerbations of COPD. Chest, 2011. 140(5): p. 1177-83.

11. Tabak, Y.P., et al., Development and validation of a mortality risk-adjustment model for patients hospitalized for exacerbations of chronic obstructive pulmonary disease. Med Care, 2013. 51(7): p. 597605.

12. Almagro, P., et al., Short- and medium-term prognosis in patients hospitalized for COPD exacerbation: the CODEX index. Chest, 2014. 145(5): p. 972-980.

13. Celli, B.R., et al., The body-mass index, airflow obstruction, dyspnea, and exercise capacity index in chronic obstructive pulmonary disease. N Engl J Med, 2004. 350(10): p. 1005-12.

14. Puhan, M.A., et al., Expansion of the prognostic assessment of patients with chronic obstructive pulmonary disease: the updated BODE index and the ADO index. Lancet, 2009. 374(9691): p. 704-11.

15. Abu Hussein, N., et al., The ADO index as a predictor of two-year mortality in general practice-based chronic obstructive pulmonary disease cohorts. Respiration, 2014. 88(3): p. 208-14.

16. Chang, C.L., et al., Predicting early mortality in acute exacerbation of chronic obstructive pulmonary disease using the CURB65 score. Respirology, 2011. 16(1): p. 146-51.

17. van Walraven, C., et al., Derivation and validation of an index to predict early death or unplanned readmission after discharge from hospital to the community. CMAJ, 2010. 182(6): p. 551-7.

18. Echevarria, C., et al., The PEARL score predicts 90-day readmission or death after hospitalisation for acute exacerbation of COPD. Thorax, 2017. 72(8): p. 686-693.

19. Quintana, J.M., et al., Predictive score for mortality in patients with COPD exacerbations attending hospital emergency departments. BMC Med, 2014. 12: p. 66.

20. Esteban, C., et al., Development of a decision tree to assess the severity and prognosis of stable COPD. Eur Respir J, 2011. 38(6): p. 1294-300.

21. Esteban, C., et al., A decision tree to assess short-term mortality after an emergency department visit for an exacerbation of COPD: a cohort study. Respir Res, 2015. 16: p. 151.

22. Vogelmeier, C.F., et al., Global Strategy for the Diagnosis, Management, and Prevention of Chronic Obstructive Lung Disease 2017 Report. GOLD Executive Summary. Am J Respir Crit Care Med, 2017. 195(5): p. 557-582.

23. Breiman Leo, F.J.H., Olshen Richard A., Stone Charles J. , Classification and regression trees. Wadsworth, California, 1984.

24. Garcia-Sanz, M.T., et al., One-year and long-term mortality in patients hospitalized for chronic obstructive pulmonary disease. J Thorac Dis, 2017. 9(3): p. 636-645. 
25. Kumar, P., S. Law, and K.B. Sriram, Evaluation of platelet lymphocyte ratio and 90-day mortality in patients with acute exacerbation of chronic obstructive pulmonary disease. J Thorac Dis, 2017. 9(6): p. 1509-1516.

26. Roberts, C.M., et al., Acidosis, non-invasive ventilation and mortality in hospitalised COPD exacerbations. Thorax, 2011. 66(1): p. 43-8.

27. Matkovic, Z., et al., Predictors of adverse outcome in patients hospitalised for exacerbation of chronic obstructive pulmonary disease. Respiration, 2012. 84(1): p. 17-26.

28. Roche, N., et al., Predictors of outcomes in COPD exacerbation cases presenting to the emergency department. Eur Respir J, 2008. 32(4): p. 953-61.

29. Tabak, Y.P., et al., Mortality and need for mechanical ventilation in acute exacerbations of chronic obstructive pulmonary disease: development and validation of a simple risk score. Arch Intern Med, 2009. 169(17): p. 1595-602.

30. Singanayagam, A., S. Schembri, and J.D. Chalmers, Predictors of mortality in hospitalized adults with acute exacerbation of chronic obstructive pulmonary disease. Ann Am Thorac Soc, 2013. 10(2): p. 81-9.

31. Arostegui, I., et al., Subtypes of patients experiencing exacerbations of COPD and associations with outcomes. PLoS One, 2014. 9(6): p. e98580.

32. Steer, J., et al., Dyspnoea severity and pneumonia as predictors of in-hospital mortality and early readmission in acute exacerbations of COPD. Thorax, 2012. 67(2): p. 117-21.

33. Soltani, A., et al., Prospective outcomes in patients with acute exacerbations of chronic obstructive pulmonary disease presenting to hospital: a generalisable clinical audit. Intern Med J, 2015. 45(9): p. 925-33.

34. Haidri, F.R., N. Rizvi, and B. Motiani, Role of APACHE score in predicting mortality in chest ICU. J Pak Med Assoc, 2011. 61(6): p. 589-92.

35. Feng, Z., et al., Efficacy of Various Scoring Systems for Predicting the 28-Day Survival Rate among Patients with Acute Exacerbation of Chronic Obstructive Pulmonary Disease Requiring Emergency Intensive Care. Can Respir J, 2017. 2017: p. 3063510.

36. Hakim, M.A., et al., Performance of the LACE index to predict 30-day hospital readmissions in patients with chronic obstructive pulmonary disease. Clin Epidemiol, 2018. 10: p. 51-59.

37. Landbo, C., et al., Prognostic value of nutritional status in chronic obstructive pulmonary disease. Am J Respir Crit Care Med, 1999. 160(6): p. 1856-61.

38. Lainscak, M., et al., Body mass index and prognosis in patients hospitalized with acute exacerbation of chronic obstructive pulmonary disease. J Cachexia Sarcopenia Muscle, 2011. 2(2): p. 81-86.

39. Schols, A.M., et al., Weight loss is a reversible factor in the prognosis of chronic obstructive pulmonary disease. Am J Respir Crit Care Med, 1998. 157(6 Pt 1): p. 1791-7.

40. Santibanez, M., et al., Predictors of Hospitalized Exacerbations and Mortality in Chronic Obstructive Pulmonary Disease. PLoS One, 2016. 11(6): p. e0158727.

41. Wouters, E.F., The burden of COPD in The Netherlands: results from the Confronting COPD survey. Respir Med, 2003. 97 Suppl C: p. S51-9.

42. Lieberman, D., et al., Pneumonic vs nonpneumonic acute exacerbations of COPD. Chest, 2002. 122(4): p. 1264-70.

43. Quintana, J.M., et al., Predictors of hospital admission two months after emergency department evaluation of COPD exacerbation. Respiration, 2014. 88(4): p. 298-306.

44. Charlson, M.E., et al., A new method of classifying prognostic comorbidity in longitudinal studies: development and validation. J Chronic Dis, 1987. 40(5): p. 373-83.

45. Yohannes, A.M., R.C. Baldwin, and M. Connolly, Mortality predictors in disabling chronic obstructive pulmonary disease in old age. Age Ageing, 2002. 31(2): p. 137-40.

46. Schmidt, S.A., et al., The impact of exacerbation frequency on mortality following acute exacerbations of COPD: a registry-based cohort study. BMJ Open, 2014. 4(12): p. e006720.

47. Triest, F.J., et al., Poor agreement between chart-based and objectively identified comorbidities of COPD. Eur Respir J, 2015. 46(5): p. 1492-5.

48. Mannino, D.M., D.E. Doherty, and A. Sonia Buist, Global Initiative on Obstructive Lung Disease (GOLD) classification of lung disease and mortality: findings from the Atherosclerosis Risk in Communities (ARIC) study. Respir Med, 2006. 100(1): p. 115-22. 
49. Lewis, R.J. An introduction to Classification and Regression Tree (CART) analysis. in Proceedings of Annual Meeting of the Society for Academic Emergency Medicine. 2000. San Francisco, CA, USA.

50. McConnochie, K.M., K.J. Roghmann, and J. Pasternack, Developing prediction rules and evaluating observation patterns using categorical clinical markers: two complementary procedures. Med Decis Making, 1993. 13(1): p. 30-42.

51. Vestbo, J., et al., Global strategy for the diagnosis, management, and prevention of chronic obstructive pulmonary disease: GOLD executive summary. Am J Respir Crit Care Med, 2013. 187(4): p. 347-65.

52. Sogaard, M., et al., Incidence and outcomes of patients hospitalized with COPD exacerbation with and without pneumonia. Int J Chron Obstruct Pulmon Dis, 2016. 11: p. 455-65. 



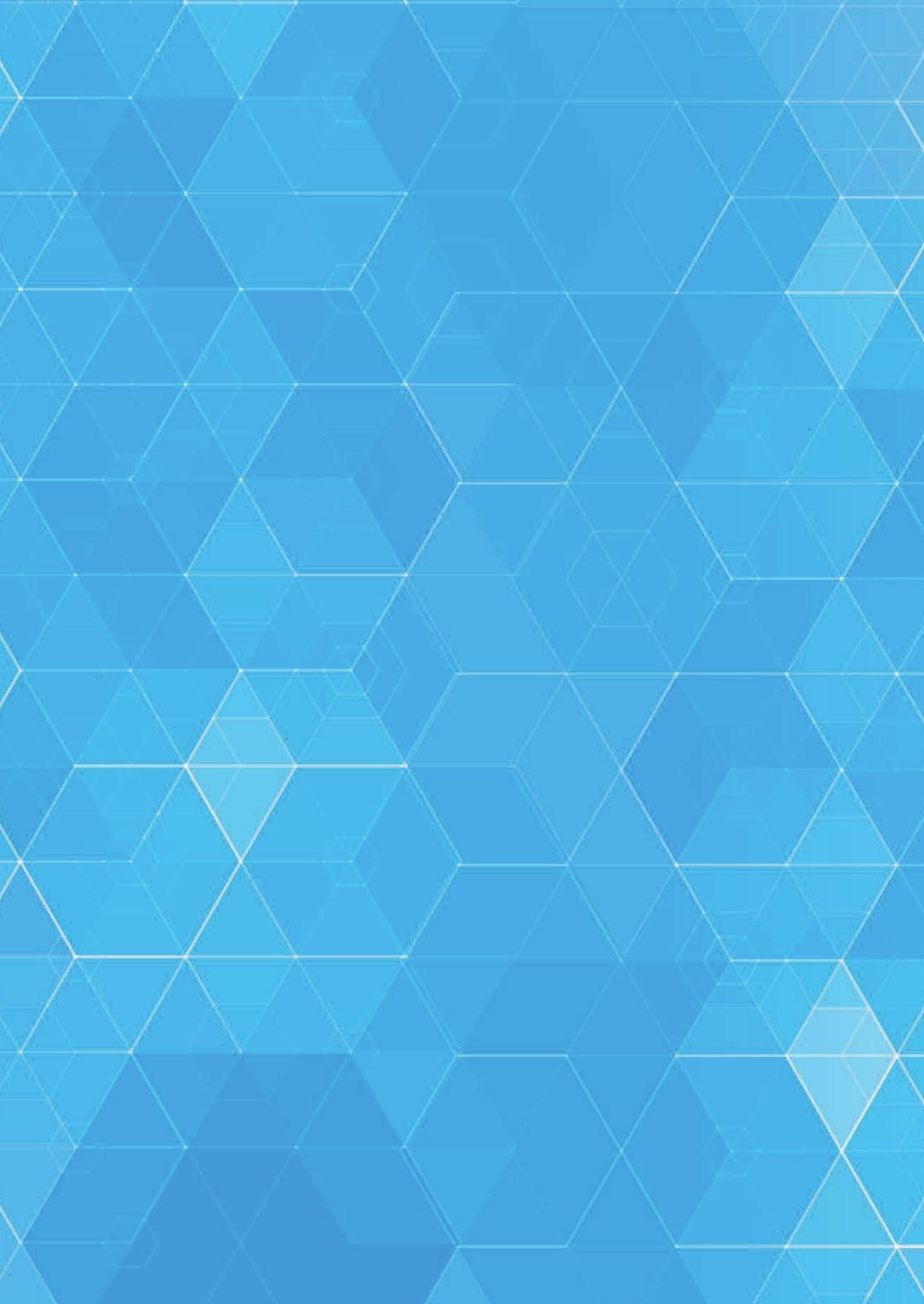




\section{Chapter 6}

Predictors for long-term mortality in COPD patients requiring non-invasive positive pressure ventilation for the treatment of acute respiratory failure

Roy T.M. Sprooten, Gernot G.U. Rohde, M.T.H.F. Janssen, Nicolle A.M. Cobben, Emiel F.M. Wouters, Frits M.E. Franssen Published in Clinical Respiratory Journal 2020;14:1144-1152 


\section{Abstract}

\section{Introduction}

The effectiveness of non-invasive mechanical ventilation (NIV) in the management of COPD patients suffering from acute respiratory failure (ARF) as a consequence of exacerbation of the disease, is well-established. However, data on long-term outcomes and their predictors, including the individual response to NIV, are scarce. The aim of the study was to investigate predictors for short- and long-term mortality in this study population.

\section{Methods}

A retrospective cohort study was performed including all patients admitted to the Medium Respiratory Care Unit of Maastricht University Medical Center in Maastricht, the Netherlands, with hospitalized exacerbation of COPD (H-ECOPD) with ARF requiring NIV for the first time between January 2009 and December 2011. An extensive number of potential predictors of outcomes, including the response to NIV, were determined on admission and during hospitalization. Univariate and multivariate logistic regression was used for statistical analysis.

\section{Results}

Seventy-eight consecutive patients with moderate to severe COPD (mean age $71.0 \pm 10.7$ y; $48.7 \%$ males) were included; In-hospital, one-year and 2-year mortality rates were $14.1 \%, 43.6 \%$ and $56.4 \%$, respectively. Independent risk factors for 2-year mortality were: advanced age (odds ratio(OR)1.025; confidence interval (Cl): 1.002-1.049; $p=0.037$ ), prolonged NIV use more than 8 days (OR: $1.054 ; \mathrm{Cl}: 1.006-1.104$; $p=0.027$ ) and no successful response to NIV (OR: 2.392; $\mathrm{Cl}: 1.297-4.413 ; \mathrm{p}=0.005)$.

\section{Conclusions}

Patients with a H-ECOPD requiring NIV for the first time, constitute a severely ill patient group with high in-hospital and two-year mortality. This study identified advanced age, NIV use more than 8 days and unsuccessful response to NIV as clinical important independent predictors for long-term mortality. 


\section{Introduction}

Severe exacerbations, defined as those requiring hospitalization, are key drivers of healthcare utilization and costs in patients with chronic obstructive pulmonary disease (COPD). ${ }^{1}$ Also, they are associated with accelerated lung function decline ${ }^{2}$ and with poor survival. ${ }^{3}$ While in-hospital mortality rates vary between $6-11 \%{ }^{4-7}$, one-year mortality ranges from 23-43\%. ${ }^{5,6}$ Advanced age, low body mass index (BMI), chronic hypercapnia and a history of severe exacerbations are well-recognized independent risk factors for mortality following these events. ${ }^{8,9}$ Also, the presence of cor pulmonale or congestive heart failure ${ }^{5}$ and the long-term use of oral corticosteroids ${ }^{6}$ are associated with reduced survival following severe exacerbations. ${ }^{5}$

Twenty to thirty percent of patients with severe exacerbations have acute or acute-onchronic hypercapnic respiratory failure ${ }^{4,7}$, for which non-invasive mechanical ventilation (NIV) is recommended. NIV reduces the need for intubation, mortality, complications of therapy, and length of both hospital stay and intensive care unit (ICU) stay. ${ }^{10}$ However, in-hospital and short-term mortality in patients receiving NIV is substantially higher compared to those not requiring ventilatory support, reflecting the increased severity of the exacerbation of COPD (ECOPD). ${ }^{11-15}$ Few studies investigated predictors of mortality in patients receiving NIV. ${ }^{12,16-18}$ Older age and low albumin ${ }^{16}$, and prior domiciliary oxygen use ${ }^{17}$ were identified as the strongest predictors for poor outcome in this population. ${ }^{16}$ However, only a limited number of both patient and exacerbation characteristics as well as details of the NIV intervention were investigated in these studies. Identification of predictors for poor outcome, will aid clinicians and their patients in (shared) decision making regarding the application and duration of continuation of NIV in severe exacerbations with acute or acute-on-chronic respiratory failure.

The current study was designed to investigate predictors for short- and long-term mortality in COPD patients requiring NIV for the treatment of acute respiratory failure related to ECOPD for the first time in the course of their disease.

\section{Methods}

A retrospective, observational cohort study was performed at the Respicare, the respiratory medium care unit of the department of respiratory medicine of Maastricht University Medical Center (MUMC) in Maastricht, the Netherlands. Clinical data of 
patients fulfilling the study criteria between January 1, 2009 and December 31, 2011 were included. Inclusion criteria were: (i) ECOPD, defined as sudden increase in one or more of the following; dyspnoea, cough or sputum production and treatment with systemic glucocorticoids and/or antibiotics, (ii) confirmation of obstructive lung function by a ratio of post-bronchodilator forced expiratory volume in 1 second to forced vital capacity $\left(\mathrm{FEV}_{1} / \mathrm{FVC}\right)<70 \%{ }^{19}$ in the medical records of the patient and (iii) requiring NIV for the first time assessed by a chest physician according to international guidelines: $\mathrm{pH}<7.35, \mathrm{PaCO}_{2}>6.5 \mathrm{kPa}$, respiratory rate $\left.(\mathrm{RR})>23 / \mathrm{min}\right) .{ }^{20}$ The index admission was the first hospitalization for acute respiratory failure (ARF) requiring NIV. The study protocol was reviewed and approved by the Medical Ethics Committee (MUMC, METC 14-04-008) and conducted according to the Declaration of Helsinki (59 ${ }^{\text {nd }}$ WMA General Assembly, Seoul, October 2008) and Good Clinical Practice guidelines. Besides optimal supportive medical treatment, including maximal bronchodilation, systemic corticosteroids and, if applicable, antibiotics, NIV was initiated in all patients. ${ }^{19,20}$ During NIV treatment, settings were adjusted according to the needs of the patients and guided by blood gas values. Demographic, clinical also including NIV related variables and laboratory parameters were retrospectively collected from the electronic records of the patients. The presence of comorbidities was recorded from the medical records according to the following categories: cardiovascular diseases; diabetes mellitus and metabolic diseases; cancers; cognitive and psychological disturbances; renal failure and urogenital tract diseases; infectious diseases and immunological diseases; musculoskeletal diseases and gastro-intestinal tract diseases. Response to NIV treatment was considered successful if patients fulfilled all of the following criteria: (i) normalization of $\mathrm{pH}>7.35$, (ii) 2) decrease of $\mathrm{PaCO}_{2}<6.0 \mathrm{kPa}$, (iii) good tolerance to NIV and (iv) no clinical requirement for intubation. During NIV treatment, the tolerance was evaluated with the patient on a daily basis and routinely registered in electronic health records of the patient. NIV treatment was considered prolonged if it was indicated for more than 8 days. In-hospital mortality was defined as mortality between admission and discharge from the hospital. Mortality during postexacerbation inpatient rehabilitation or during stay on an external weaning unit was excluded from this definition. During two year follow-up, data of discharge to home or other residency, all-cause mortality as well as readmissions were registered. Univariate analyses were performed using the Mann-Whitney $U$ test or T-test for continuous variables and the Chi square test for categorical data. Cox logistic regression (backward stepwise likelihood ratio) was used for multivariate analyses. A $p \leq 0.05$ was considered significant. For 2-year mortality variables that were significant in the univariate analyses or clinically important were entered into the multivariate analyses. Survival was analysed using the Kaplan-Meier method. 
(See supplementary information, Appendix S6.1 - Material and methods)

\section{Results}

\section{Patient characteristics}

Between 2009 and 2011, 1088 patients were admitted to the Respicare Unit. The flow chart for patient selection is depicted in Figure 6.1. Seventy-eight COPD patients with severe exacerbation requiring firstly NIV for ARF were included into our analysis. The clinical characteristics of the study population are shown in tables 1-2 and detailed information can be find in the supplemental data (Table S6.1-S6.4).

The median length of hospital stay was 16.5 (11.0-28.3) days. After discharge, 49 patients went home, 16 were admitted to a rehabilitation center or to a nursery home. Four patients went home with palliative care. Four patients received directly after admission home mechanical ventilation as non-invasive ventilation, whereas is 11 (14.1) patients long-term oxygen therapy was initiated. The readmission rate was respectively $20.8 \%$ at 90 days, $39.8 \%$ at one year and $41 \%$ after 2 years of follow-up. Data in detail about readmissions are shown in the supplemental data (Table S6.5).

Figure 6.1 Flowchart of patient selection.

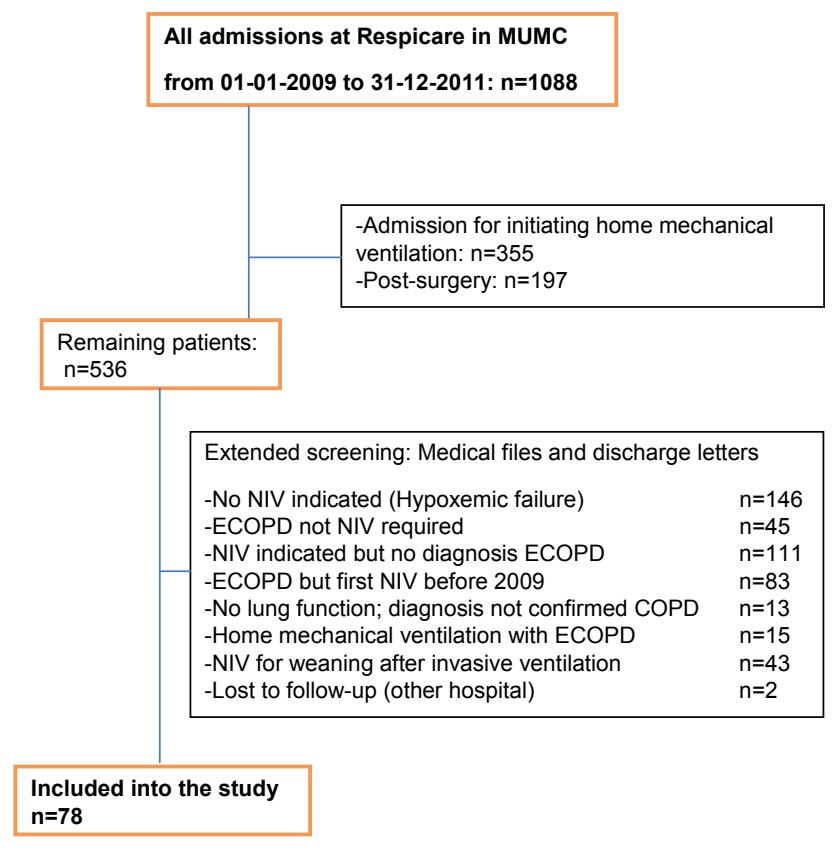


Table 6.1 Characteristics of patients hospitalized for ECOPD with acute respiratory failure and requiring non-invasive ventilation at first, stratified by in-hospital and long-term survival.

\begin{tabular}{lcccc}
\hline & $\begin{array}{c}\text { In-hospital } \\
\text { survivors } \\
(\mathbf{n}=\mathbf{6 7})\end{array}$ & $\begin{array}{c}\text { In-hospital } \\
\text { deaths } \\
(\mathbf{n}=\mathbf{1 1})\end{array}$ & $\begin{array}{c}\text { Long-term } \\
\text { survivors } \\
(\mathbf{n}=\mathbf{3 4})\end{array}$ & $\begin{array}{c}\text { Long-term } \\
\text { deaths } \\
(\mathbf{n}=\mathbf{4 4})\end{array}$ \\
\hline Male gender & $30(44.8)$ & $8(72.7)$ & $11(28.9)^{*}$ & $27(71.1)^{*}$ \\
Age at admission, $\mathrm{y}$ & $69.9 \pm 10.8^{*}$ & $77.5 \pm 7.3^{*}$ & $66.9 \pm 11.6^{*}$ & $74.1 \pm 8.9^{*}$ \\
Body mass index, $\mathrm{kg} / \mathrm{m}^{2}$ & $23.9 \pm 5.4^{*}$ & $20.6 \pm 2.2^{*}$ & $24.8 \pm 5.6$ & $22.3 \pm 4.7$ \\
Pack years $(\mathrm{n}=32 / 29 / 3)$ & $42.5 \pm 12.1$ & $53.3 \pm 11.5$ & $39.2 \pm 12.4^{*}$ & $48.9 \pm 10.2^{*}$ \\
Post-BD FEV $1, \% p r e d$ & $40.1(28.8-53.6)$ & $36.1(27.8-44.5)$ & $40.2(29.9-54.3)$ & $36.9(27.2-49.8)$ \\
Frequent exacerbators $(>1)$ & $9(13.4)$ & $2(18.2)$ & $4(11.8)$ & $7(15.9)$ \\
CCl & $2.0(1-3)^{*}$ & $3.0(2-4)^{*}$ & $2(1-3)$ & $2(1-3)$ \\
\hline
\end{tabular}

Categorical data are presented as $n(\%)$, non-parametric data as median (IQR) and parametric data as mean \pm (SD). Chi- $\mathrm{X}^{2}$, Kruskal-wallis test and T-test were used for statistical analysis respectively. ${ }^{*} \mathrm{p}$-value $<0.05$; ${ }^{\#}$ p-value <0.001. Abbrevations: Post-BD: post bronchodilator; FEV1: forced expiratory volume in the first second; $\mathrm{CCl}$ : Charlson Comorbidity Index.

\section{Predictors of mortality}

In-hospital mortality was $14,1 \%(n=11)$. Mortality rate during follow-up was respectively, $29.5 \%$ at 90 days, $43.6 \%$ at one year and $56.4 \%$ at two years. Most of the deaths died because of respiratory failure due to ECOPD (see Figure 6.2).

Results of the univariate comparison between the in-hospital survivors and nonsurvivors are shown in Tables 6.1-6.2. Significant predictors are: advanced age at admission, smoking status, prednisone maintenance therapy, high $\mathrm{CCl}$ especially gastrointestinal tract involvement, low BMI, Blood urea nitrogen $(\mathrm{BUN}) \geq 8 \mathrm{mmol} / \mathrm{L}^{4}$, high troponin levels $(n=38)$, lower $\mathrm{pH}$ before start NIV, bicarbonate $\geq 27.0 \mathrm{mmol} / \mathrm{L}$, changed limited treatment restriction at admission. After one hour of NIV, RR was significant higher in deceased patients. Moreover, the $\mathrm{pH}$ and $\mathrm{PaCO}_{2}$ and the changes between the time points 1, 24 and 72 hours did not show any significant differences between the groups. In 43 (55.1\%) patients NIV was assessed as successful. NIV was successful in 3 out of 11 in-hospital non-survivors compared to 40 out of 67 survivors $(27.3 \%$ vs. $59.7 \%, p=0.045$ (pearson Chi-Square test). The survivor group had a significantly better tolerance to NIV compared to the non-survivors ( $80.6 \%$ vs. $12.5 \%, p=0.0001$ (pearson Chi-square test)). There was no significant difference in the percentage of patients with successful response between non-survivors and survivors after two years follow up (see supplementary information Table S6.4). 
Table 6.2 Non-invasive ventilation (NIV) results of patients hospitalized for ECOPD with acute respiratory failure and requiring NIV at first, stratified by in-hospital and long-term survival.

\begin{tabular}{|c|c|c|c|c|}
\hline & $\begin{array}{c}\text { In-hospital } \\
\text { survivors } \\
(n=67)\end{array}$ & $\begin{array}{c}\text { In-hospital } \\
\text { deaths } \\
(n=11)\end{array}$ & $\begin{array}{l}\text { Long-term } \\
\text { survivors } \\
(n=34)\end{array}$ & $\begin{array}{l}\text { Long-term } \\
\text { deaths } \\
(n=44)\end{array}$ \\
\hline \multicolumn{5}{|l|}{ ABG before start NIV $(n=77)$} \\
\hline $\mathrm{pH}$ & $7.28(7.22-7.31)^{*}$ & $7.24(7.1-7.26)^{*}$ & $7.28(7.23-7.31)^{*}$ & 7.25 (7.18-7.29)* \\
\hline$<7.25$ & $21(31.8)^{*}$ & $7(63.6)^{*}$ & $8(23.5)^{*}$ & $20(46.5)^{*}$ \\
\hline $7.25-7.35$ & $45(68.2)^{*}$ & $4(36.4)^{*}$ & $26(76.5)^{*}$ & $23(53.5)^{*}$ \\
\hline $\mathrm{PaCO}_{2}, \mathrm{kPa}$ & $10.0(8.5-11.0)$ & $10.8(8.3-12.1)$ & $9.7(8.5-10.5)$ & $10.3(8.6-11.5)$ \\
\hline $\mathrm{HCO}_{3}{ }^{-}, \mathrm{mmol} / \mathrm{L}$ & $31.9(28.0-36.2)^{*}$ & $26.7(25.4-36.8)^{*}$ & $31.4(27.8-73.0)$ & $31.8(26.7-36.0)$ \\
\hline Base excess & $3.7(-0.3-6.6)$ & $2.9(-5.4-6.6)$ & $3.9(-0.3-7.0)$ & $3.1(-2.6-6.5)$ \\
\hline$<-2.5$ & $9(13.6)^{*}$ & $5(45.5)^{*}$ & $3(8.8)$ & $11(25.6)$ \\
\hline$-2.5-2.5$ & $21(31.8)^{*}$ & $0^{*}$ & $12(35.3)$ & 9 (20.9) \\
\hline$>2.5$ & $36(54.5)^{*}$ & $6(54.5)^{*}$ & $19(55.9)$ & $23(53.5)$ \\
\hline \multicolumn{5}{|l|}{ Clinical data } \\
\hline LOS, days & $17.0(11.0-27.0)$ & $11.0(3.0-31.0)$ & 13.5 (10.0-18.3) & $17.0(8.3-28.0)$ \\
\hline NIV, days & $5.0(2.0-7.0)$ & $7.0(3.0-11.0)$ & $4.0(3.0-6.0)$ & $5.0(2.0-10.8)$ \\
\hline NIV $>8$ days & $11(16.4)$ & $4(36.4)$ & $3(8.8)^{*}$ & $12(27.3)^{*}$ \\
\hline IPAP, $\mathrm{cm} \mathrm{H}{ }_{2} \mathrm{O}$ & $20.5 \pm 4.3$ & $22.9 \pm 3.8$ & $21.2 \pm 3.9$ & $20.6 \pm 4.5$ \\
\hline EPAP, $\mathrm{cm} \mathrm{H}_{2} \mathrm{O}$ & $6.2 \pm 1.5$ & $7.1 \pm 1.4$ & $6.3 \pm 1.3$ & $6.4 \pm 1.7$ \\
\hline \multicolumn{5}{|l|}{ NIV response after $1 \mathrm{~h}$} \\
\hline $\begin{array}{l}\text { Delta } \mathrm{pCO}_{2} \text { before NIV-1h, } \mathrm{kPa} \\
\mathrm{pH} \geq 7.35 \text { at } 1 \mathrm{~h}\end{array}$ & $1.4 \pm 1.3$ & $1.6 \pm 1.1$ & $1.1 \pm 1.1$ & $1.6 \pm 1.3$ \\
\hline $\mathrm{RR}$ at $1 \mathrm{~h}, / \mathrm{min}$ & $21(31.8)$ & $1(9.1)$ & $12(35.3)$ & $10(23.3)$ \\
\hline \multirow[t]{2}{*}{$\mathrm{RR}>20 / \mathrm{min}$ at $1 \mathrm{~h}$} & $22.2 \pm 5.9 *$ & $26.7 \pm 4.4^{*}$ & $21.0 \pm 6.1^{*}$ & $24.3 \pm 5.4^{*}$ \\
\hline & $31(57.4)$ & $8(88.9)$ & $14(48.3)^{*}$ & $25(73.5)^{*}$ \\
\hline
\end{tabular}

Categorical data are presented as $\mathrm{n}(\%)$, non-parametric data as median (IQR) and parametric data as mean \pm (SD). Chi- $X^{2}$, Kruskal-wallis test and T-test were used for statistical analysis respectively. ${ }^{*} p$-value $<0.05 ;{ }^{\#} p$ value <0.001. Abbrevations: ABG: arterial blood gas; LOS: Length of hospital stay; IPAP inspiratory positive air pressure; EPAP: Expiratory positive air pressure; RR: respiratory rate; BE: base excess; NIV: non-invasive ventilation.

The univariate comparison between the survivors and non-survivors after 2-year follow-up are presented in Tables 6.1 and 6.2. Prior to NIV, several significant negative predictors were found: male gender, higher amount of pack years, prednisone maintenance therapy, atrial fibrillation, low $\mathrm{pH}$, change in medical policy restrictions. $\mathrm{RR}$ after 1 hour use of NIV was significantly higher in the non-survivors. The delta $\mathrm{PaCO}_{2}$ before start and at termination NIV was different but failed to reach statistical significance $(p=0.058)$.

In the multivariate analysis were entered the following clinically relevant or significant univariate analysis variables: advanced age, gender, $\mathrm{pH}$ before start NIV, RR >20min, delta $\mathrm{paCO}_{2}$ before start NIV vs at termination NIV, duration NIV (days), response (tolerance) to NIV. As independent factors associated with 2-year mortality advanced age, prolonged (>8 days) NIV use and the response to NIV assessed to be unsuccessful 
were identified (Table 6.3). Figure 6.3 shows the Kaplan Meier survival curve of prolonged (>8 days) NIV use (Logrank 0.025; Breslow 0.040), unsuccessful NIV (Logrank 0.011; Breslow 0.002) and occurrence of readmission after discharge (Logrank 0.0001; Breslow 0.0001), which have negative impact on survival.

(See supplementary information, Appendix S6.2-Results)

Figure 6.2 Cause of death during 2 year follow-up in patients cohort hospitalized for ECOPD with acute respiratory failure and requiring non-invasive ventilation at first. $(n(\%))$.

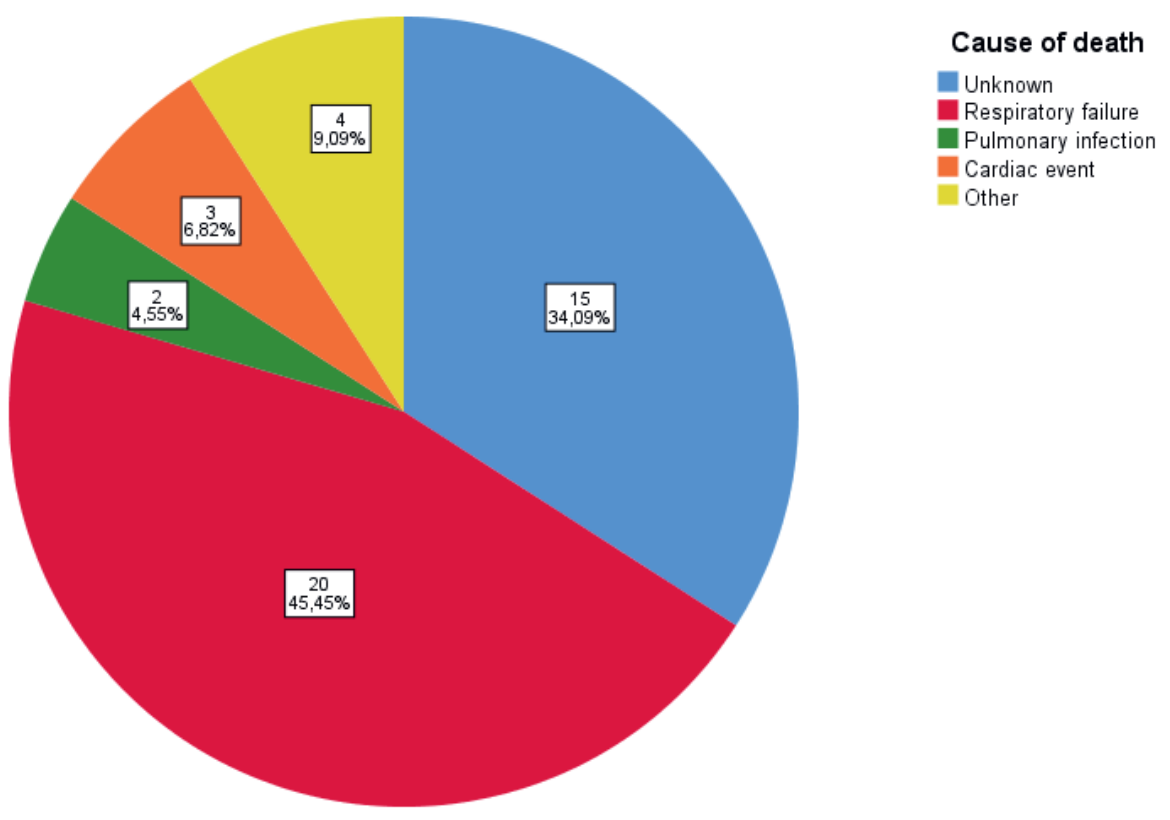


Figure 6.3 Overall mortality after two years follow-up of patients hospitalized for ECOPD with acute respiratory failure and requiring non-invasive ventilation at first, stratified by respectively days treated with NIV ( $>8$ days or $\leq 8$ days), see figure $A,(n=78$; Logrank 0.025, Breslow 0.040, Pearson Chi- $\mathrm{X}^{2}$ 0.040); successful NIV (home mechanical ventilation is regarded as successful) (yes or no), see figure $B,(n=78$; Log rank 0.011 , Breslow 0.002 ) and readmission during followup, see figure C, ( $n=67$; Log rank 0.0001, Breslow 0.0001). Abbrevations: $d$ : days.
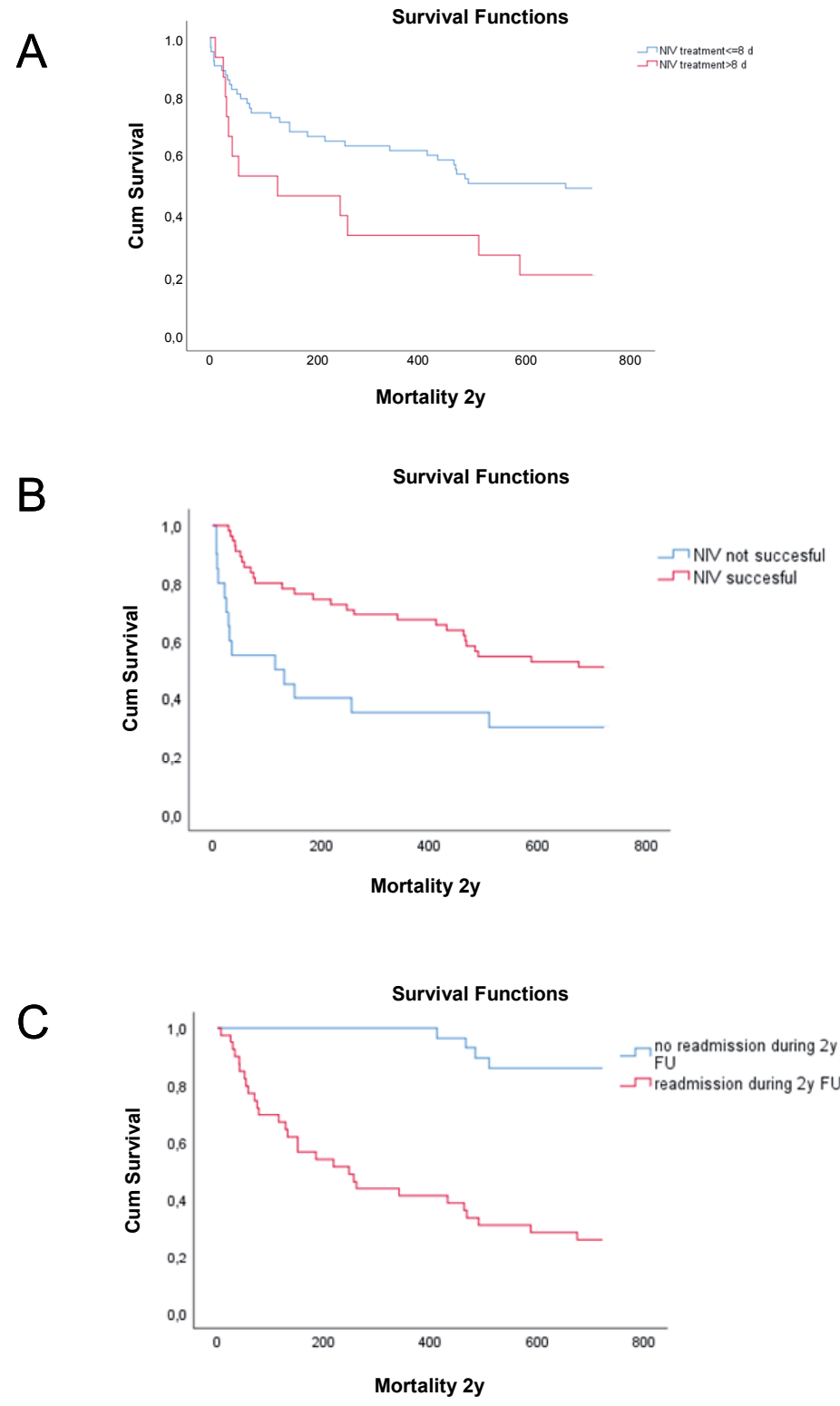
Table 6.3 Multivariate model using 7 coefficients to analyse two years mortality in patients hospitalized for ECOPD with acute respiratory failure and requiring non-invasive ventilation at first. Shown in table is the predicted odds ratio (OR) based on the regression coefficient Beta, the $95 \%$ confidence interval for Beta and the uncorrected p-value using the backward stepwise cox regression. All data available in 60 subjects.

\begin{tabular}{|c|c|c|c|}
\hline & OR & 95\% Cl for $\operatorname{Exp}(\mathrm{B})$ & p-value \\
\hline Age* (years) & 1.025 & $1.002-1.049$ & 0.037 \\
\hline Gender ${ }^{\#}($ female $=0)$ & 0.808 & $0.437-1.382$ & 0.436 \\
\hline $\mathrm{pH}$ before start NIV* & 0.136 & $0.002-11.478$ & 0.378 \\
\hline $\mathrm{RR}>20 / \mathrm{min}^{\#}(>20 / \mathrm{min}=0)$ & 0.945 & $0.528-1.692$ & 0.850 \\
\hline Delta $\mathrm{PaCO}_{2}$ before NIV- stop NIV $(\mathrm{kPa})^{*}$ & 1.000 & $0.972-1.029$ & 0.986 \\
\hline Total days NIV* (days) & 1.054 & $1.006-1.104$ & 0.027 \\
\hline Response tolerance/ adherence $\mathrm{NIV}^{\#}$ (not successful=0) & 2.392 & $1.297-4.413$ & 0.005 \\
\hline
\end{tabular}

${ }^{*}$ Continues variable; ${ }^{*}$ categorical variable. Abbreviations: NIV: Non-invasive ventilation; RR: respiratory rate. kPa: kilopascal; OR: odds ratio; $\mathrm{Cl}$ : confidence interval.

\section{Discussion}

This study investigated the demographic, biochemical, clinical and intervention-related predictors of short- and long-term mortality in patients requiring non-invasive positive pressure ventilation for the treatment of acute respiratory failure. Fourteen percent of patients died during hospital stay, while $56 \%$ died within two years. Older age, NIV use more than 8 days and non-successful response to NIV were identified as independent predictors for long-term mortality.

There is a large variation in the reported in-hospital mortality rates for patients with severe exacerbations and need for NIV. Differences in study population, severity of ECOPD and clinical setting contribute to this variation. Some studies reported low mortality rates of $4-5 \% .{ }^{13,16,21}$ A large nationwide study in the United States of America published by Chandra et al. found a mortality rate of $9 \%$ in patients with ECOPD and ARF who only needed NIV. ${ }^{11}$ Plant et al, one of the first randomized trials in COPD and NIV, reported an in-hospital mortality of $10 \%$ in the NIV group. ${ }^{22}$ Another study in patients managed in intermediate care unit reported a mortality rate of $20 \%{ }^{23}$ Sainaghi et al reported mortality rates of patients admitted with ECOPD and ARF requiring NIV managed in-ward as high as $27 \% .{ }^{24}$ Patients with ECOPD and ARF started with NIV and transitioned to invasive mechanical ventilation (IMV) had a worse mortality rate up to $27 \% .{ }^{11}$ In a prospective study mortality rate was studied in patients admitted to respiratory intensive care unit with ECOPD and acute hypercapnic respiratory failure. From the 151 enrolled patients eighty seven (57.6\%) received mechanical ventilation and 22 patients (14.6\%) NIV. The in-hospital mortality was $33.1 \%{ }^{25}$ The authors 
attributed the higher mortality rate to higher severity of illness and higher intubation rates.

In our study the in-hospital mortality rate is $14.1 \%$ which is higher than several studies reported. ${ }^{13,16,21,26}$ This difference can be explained by the severity of ARF and the severity of illness, reflected in lower $\mathrm{pH}$ levels in our study population. Still the inhospital mortality we found in our study is much lower in patients with hospitalizedECOPD (H-ECOPD) and ARF intubated and required IMV (25\%). ${ }^{27}$

There is limited data about long-term mortality in patients admitted for ECOPD with acute respiratory failure requiring NIV and results are again variable. In a randomized controlled study by Plant et al the 1-year survival in the NIV group was $61.6 \%{ }^{22} \mathrm{~A}$ retrospective single center study in Spain reported a long-term mortality at 1,2 and 3 years respectively as $30 \%, 47.7 \%$ and $74 \% .{ }^{16}$ Chung et al investigated the long-term mortality in patients admitted for ECOPD with acute respiratory failure firstly requiring NIV and found a one-year mortality of $28 \%$, a two-year mortality of $48 \%$ and a five-year mortality of $74 \% .{ }^{17}$ Another study published by Titlestad showed comparable outcomes. ${ }^{12}$ Our study revealed a two-year mortality rate of $56.4 \%$. The slightly higher mortality rates at one and two years in our study compared to the study from Chung et al can be explained by the fact that Chung only included patients who survived the initial hospital admission whereas in our study we analysed all patients admitted.

Identifying factors influencing in-hospital and long-term mortality is pivotal in management of these severely ill patients, as NIV may be stressful and demanding and adverse events may occur. Therefore, initiation and continuation of NIV treatment is not only a decision by the pulmonologist, but also a moment of shared-decision making with the patient and his relatives. This discussion benefits from well-established predictors for short- and long-term outcomes. The present study observed that patients with advanced age had worse outcome, which is in concordance with previous studies. $^{4-6,9,12,15,17,26,28,29}$

Another independent predictor for 2-year mortality was prolonged use of NIV, especially longer than 8 days. This finding is comparable with findings of a study published by Penuelas. ${ }^{30}$ They found in a cohort of mechanical ventilated patients that prolonged weaning of a duration longer than 7 days is associated with an increased risk for death. However, these patients underwent IMV and not NIV. 
The present study observed that response to NIV considered as unsuccessful was found a negative independent predictor for long-term mortality. Literature investigating the meaning of the response of NIV and its impact on long-term survival after H-ECOPD is scarce. It has been reported that the more seriously ill patients, as well as those with a poorer initial response to NIV are more likely to suffer NIV failure. ${ }^{31,32}$ Previous studies revealed several factors predicting NIV failure (short-term) which are severity hypercapnia and acidosis at admission, failure in improvement first hour, diaphragmatic dysfunction, patient ventilator asynchrony, poor nutritional status and pneumonia as a cause of ARF. ${ }^{13,21,31,33-35}$ However, most studies about this subject were done in ICU populations. Plant et al demonstrated that the severity of acidosis, degree of hypercapnia, severity of hypoxia were associated with failure of NIV treatment and subsequent negative impact on mortality, while decrease in respiratory rate after 4 hours and improvement of $\mathrm{pH}$ were associated with successful outcome. ${ }^{22}$ Akyil found in their study higher white blood cell count, lower haematocrit, lower levels of albumin at admission, lower $\mathrm{pH}$, and higher $\mathrm{PaCO}_{2}$ level at 24 hours as predictors for negative outcome. ${ }^{16}$ In a more real life setting, Nicolini et al. demonstrated that patients with fewer comorbidities, less severe illness and improvement in ABG parameters within 1 hour are more likely to have successful outcome. ${ }^{21}$ Furthermore, the appropriate choice of ventilator modality and interface and the level of team experience and patient's cooperation play a pivotal role in successful NIV treatment. $^{36-38}$ These findings are in line with our study. Few studies reported factors that have negative impact on long-term survival. Titlestad et al identified advanced age and do-not-intubate orders as an independent negative predictor. ${ }^{12}$ Levels of $\mathrm{pH}$ and $\mathrm{PaCO}_{2}$ were not identified as a predictor. Another study described advanced age, lower BMI, and LTOT as long-term mortality predictors. Again this study found that ABG levels at admission, 2 hours after NIV initiation and at discharge were correlated with survival. These finding are in line with a recently published study by Steriade et al in which 89 patient with ECOPD requiring NIV were included. They reported that the mortality and NIV failure (defined as intubation or in-hospital death) was not influenced by the arterial blood gas values in the first $6 \mathrm{~h}$ of NIV. Unfortunately, the data are not sufficient to analyze the impact on long-term mortality. ${ }^{26}$

In the current study prednisone maintenance therapy was identified as a predictor of in-hospital and long-term mortality in the univariate analysis. This could be due to adverse effects of prednisone or confounding with underlying disease severity. Our results confirm the findings by Groenewegen et al, who reported that the use of oral corticosteroids was an independent risk factor for mortality after H-ECOPD. ${ }^{6}$ 
Significant findings in the univariate analysis for potential predictors of 2-year outcome were male gender, advanced age at admission, prednisone maintenance. A lower $\mathrm{pH}$ before start of NIV was also a significant predictor. Response to NIV according normalizing $\mathrm{pH}$ or lowering $\mathrm{PaCO}_{2}$ did not seek out as a significant predictor. Regarding male gender, which in our study was a predictor for mortality, several reports are published. ${ }^{4,12}$ Slenter et al also showed a prominent association of male gender with an increased risk of death and indicated that male COPD patients admitted to the hospital with ECOPD had a worse health status. ${ }^{4}$ Another study performed in patients with ECOPD and ARF requiring NIV observed a trend towards better long-term (5 years) survival in females compared to males. ${ }^{12}$ The explanation of this observation could be the historical fact that males might have had a higher exposure of noxious particles (cigarette smoke or occupational lung irritants) contributing to worse long-term survival. ${ }^{39}$ However, our data cannot support this theory.

The need of repeated hospitalizations for ECOPD is associated with poor outcomes. In our study the readmission rate in the first year was almost $40 \%$. Chung et al reported a readmission rate in the first year of follow-up of $56 \% .{ }^{17}$ This stresses the importance of post hospitalization care in this very frail patient group.

\section{Strengths and limitations}

The thorough assessment of patient demographics, clinical, biometrical and biochemical parameters and recording of details of the NIV intervention in a real-life clinical setting with long-term follow up is the strength of the current study. Also, the collected data was highly complete with few missing patients and no loss to follow-up. The diagnosis COPD was well documented and confirmed by spirometry. Furthermore, to our knowledge this is the first study that investigated the short and long-term mortality related to the response to or successful use of NIV.

Our results should be interpreted in light of some limitations. First, its retrospective design could lead to information bias. The presented study was performed in a single center which limits the generalizability of our results. Moreover, potential important prognostic factors such as the SAPS II score ${ }^{21}$, patient's activities of daily living, dyspnoea score ${ }^{40}$ could were not obtained, because they were not available. The NIV tolerance was subjectively assessed clinically by the nurses and pulmonologists. The sample size of 78 patients is relatively small and may have limited the detection of other important predictors. 


\section{Conclusions}

\section{Clinical implications and summary}

This retrospective, single-center study shows that patients who are admitted to the hospital for a first hospitalization requiring NIV for ARF in an ECOPD, are a severely ill patient group with high short and long-term mortality rates. Not the response of levels of $\mathrm{PaCO}_{2}$ or $\mathrm{pH}$ but older age, NIV use more than 8 days and lack of successful NIV response are independent prognostic factors for two-year mortality. These results could help clinicians to predict the prognosis in these patients and facilitate 'shared decision making' with these severe ill patients. Also, continuation of NIV in patients above 8 days can be debated. Obviously, further research is needed to determine the exact value of these factors to predict the prognosis in patients admitted with acute respiratory failure requiring NIV at first and its response to NIV. 


\section{Appendix S6.1- Material and Methods (extended version)}

\section{Design and population}

A retrospective, observational cohort study was performed at the Respicare, the respiratory medium care unit of the department of respiratory medicine of Maastricht University Medical Center (MUMC) in Maastricht, the Netherlands. Clinical data of patients fulfilling the study criteria between January 1, 2009 and December 31, 2011 were included. Inclusion criteria were: (i) ECOPD, defined as sudden increase in one or more of the following; dyspnea, cough or sputum production and treatment with systemic glucocorticoids and/or antibiotics, (ii) confirmation of obstructive lung function by a ratio of post-bronchodilator forced expiratory volume in 1 second to forced vital capacity $\left(\mathrm{FEV}_{1} / \mathrm{FVC}\right)<70 \%{ }^{19}$ in the medical records of the patient and (i) requiring NIV for the first time assessed by a chest physician according to international guidelines: $\mathrm{pH}<7.35, \mathrm{PaCO}_{2}>6.5 \mathrm{kPa}$, respiratory rate $\left.(\mathrm{RR})>23 / \mathrm{min}\right) .{ }^{20}$ Exclusion criteria were: (i) age $<40$ years, (ii) active pulmonary malignancy based on medical records, (iii) follow-up in another hospital and (iv) primary reason for hospitalization other than exacerbation. The index admission was the first hospitalization for acute respiratory failure (ARF) requiring NIV. The study protocol was reviewed and approved by the Medical Ethics Committee (MUMC, METC 14-04-008) and conducted according to the Declaration of Helsinki ( $59^{\text {nd }}$ WMA General Assembly, Seoul, October 2008) and Good Clinical Practice guidelines.

Besides optimal supportive medical treatment, including maximal bronchodilation, systemic corticosteroids and, in applicable, antibiotics, NIV was initiated in all patients. ${ }^{19,20}$ The initial ventilator settings (BIPAP vision, ventilator, Philips, Respironics, Netherlands, Eindhoven) were spontaneous/timed mode (S/T mode), inspiratory positive airway pressure (IPAP) to expiratory positive airway pressure (EPAP) ratio $14 / 4 \mathrm{cmH}_{2} \mathrm{O}$, backup frequency $12 / \mathrm{min}$, inspiratory time $1.0 \mathrm{~s}$, rise time $50 \mathrm{~ms}$. During NIV treatment, settings were adjusted according to the needs of the patients and guided by blood gas values. After stabilization of the patient, weaning was started.

\section{Data collection}

Demographic, clinical and laboratory parameters were retrospectively collected from the electronic records of the patients. The following variables were recorded: preadmission data such as demographics, smoking and medical history including comorbidities, previous admissions for severe exacerbations, post-bronchodilator $\mathrm{FEV}_{1}$ and FVC, FEV 1 /FVC ratio, GOLD stage for airflow limitation, residual volume (RV), total 
lung capacity (TLC), diffusion capacity (DLCO), need for home care, domestic situation, respiratory medications, use of long term oxygen treatment (LTOT) and arterial blood gases ( $A B G$ ) before hospitalization. Comorbidities were quantified using the Charlson's comorbidity index $(\mathrm{CCl}){ }^{41}$ The presence of comorbidities was recorded from the medical records according to the following categories: cardiovascular diseases; diabetes mellitus and metabolic diseases; cancers, cognitive and psychological disturbances; renal failure and urogenital tract diseases; infectious diseases and immunological diseases; musculoskeletal diseases and gastro-intestinal tract diseases. On admission and during treatment, the following data were collected: vital signs, physical examination, BMI, ABG, blood chemistry, electrocardiogram (rhythm and signs of ischemia), chest X-ray (consolidation and signs of congestion), ABG before start of NIV and after 1, 24 and 72 hours of NIV and before stop. Also, length of hospital stay was recorded. NIV related variables were collected: duration of NIV, ventilator settings such as IPAP, EPAP, reason to discontinue NIV, patient cooperation and tolerance of NIV, complications and the need for intubation. The clinical response to NIV was assessed at 1 hour, 24 and 72 hours after start NIV. Response to NIV treatment was considered successful if patients fulfilled all of the following criteria: 1 ) normalization of $\mathrm{pH}>7.35$, 2) decrease of $\mathrm{PaCO}_{2}<6.0 \mathrm{kPa}, 3$ ) good tolerance to NIV and 4) no clinical requirement for intubation. During NIV treatment, the tolerance of NIV was evaluated with the patient on a daily basis and routinely registered in electronic health records of the patient. NIV treatment was considered prolonged if it was indicated for more than 8 days. In-hospital mortality was defined as mortality between admission and discharge from the hospital. Mortality during post-exacerbation inpatient rehabilitation or during stay on an external weaning unit was excluded from this definition. During two year follow-up, data of discharge to home or other residency, all-cause mortality as well as readmissions were registered. In case of an in-hospital death the date was recorded and verified. The date of death after hospital discharge was verified from hospital records, general practitioners records or death records of the patients' residence.

\section{Statistical analysis}

SPSS version 23 software was used for all statistical analyses. First, descriptive data analysis was performed. The distribution of data was assessed by Kolmogorov-Smirnov test. Normally distributed data are reported as mean (standard deviation) whereas non-parametric data are expressed as median (interquartile range). Categorical data are presented as percentages $(n(\%))$. Univariate analyses were performed using the Mann-Whitney $U$ test or T-test for continuous variables and the Chi square test for categorical data. All variables were included into the univariate analyses. Cox logistic 
regression (backward stepwise likelihood ratio) was used for multivariate analyses. A $p \leq 0.05$ was considered significant. For 2 -year mortality variables that were significant in the univariate analyses or clinically important were entered into the multivariate analyses. Survival was analyzed using the Kaplan-Meier method. 


\section{Appendix S6.2-Results (extended version)}

\section{Patient characteristics}

Between 2009 and 2011, 1088 patients were admitted to the Respicare Unit. The flow chart for patient selection is depicted in Figure 6.1. Following the in- and exclusion criteria of this study, 78 COPD patients with severe exacerbation requiring NIV for ARF were included into our analysis. The clinical characteristics of the study population are shown in Tables S6.1-6.4 (see supplemental data). Patients were elderly males and females with moderate to very severe COPD. Almost half of all patients had been hospitalized for ECOPD in the previous 2 years and more than half were current smokers. The majority $55(73.3 \%)$ of patients received additional professional support at home. Thirty patients (38.4\%) had LTOT and comorbidities were common. The majority of patients with previous blood gas results were hypercapnic with normal $\mathrm{pH}$ at that time (Table S6.1 and 6.3 (see supplemental data)). Vital signs and laboratory results at the time of presentation at the emergency room (ER) are presented in Table 6.2 and 6.4 (see supplemental data online). The median respiratory rate was 30 (24$34) / \mathrm{min}$, the median $\mathrm{SpO}_{2}$ was $90 \%$ (82-94\%). A third of the patients had underweight, $36.4 \%$ had a $\mathrm{pH}<7.25$, the majority had a $\mathrm{PaCO}_{2}>9.1 \mathrm{kPa}$. In almost $20 \%$ treatment restrictions were present before admission; in almost half of patients restrictions were discussed and changed on ER. In 26 patients (32.1\%) the chest X-ray showed a consolidation at admission, in 10 patients (12.3\%) a pleural effusion and in 14 patients (17.3\%) signs of heart failure.

\section{Hospital admission, discharge and long term follow-up}

The median length of hospital stay was 16.5 (11.0-28.3) days. After discharge, 49 patients went home, 16 were admitted to a rehabilitation center or to a nursery home. Four patients went home with palliative care. Four patients received directly after admission home mechanical ventilation as non-invasive ventilation, whereas is 11 (14.1) patients long-term oxygen therapy was initiated. Twelve patients (15.4\%) were admitted to post-exacerbation pulmonary rehabilitation. The readmission rate was respectively $20.8 \%$ at 90 days, 39.8\% at one year and $41 \%$ after 2 years of follow-up. Data in detail about readmissions are shown in the supplemental data (Table 6.5).

\section{Predictors of mortality}

In-hospital mortality was $14,1 \%(n=11)$. Mortality rate during follow-up was respectively, $29.5 \%$ at 90 days, $43.6 \%$ at one year and $56.4 \%$ at two years. Most of the 
deaths died because of respiratory failure due to ECOPD (see Figure 6.2 in the main manuscript).

Results of the univariate comparison between the in-hospital survivors and nonsurvivors are shown in Tables S6.1-6.2 (see supplemental data). Significant predictors are: advanced age at admission, smoking status, prednisone maintenance therapy, high $\mathrm{CCl}$ especially gastro-intestinal tract involvement, low BMI, Blood urea nitrogen (BUN) $\geq 8 \mathrm{mmol} / \mathrm{L}^{4}$, high troponin levels $(\mathrm{n}=38)$, lower $\mathrm{pH}$ before start NIV, bicarbonate $\geq 27.0 \mathrm{mmol} / \mathrm{L}$, changed limited treatment restriction at admission. After one hour of NIV, RR was significant higher in deceased patients. Moreover, the $\mathrm{pH}$ and $\mathrm{PaCO}_{2}$ and the changes between the time points 1, 24 and 72 hours did not show any significant differences between the groups. In 43 (55.1\%) patients NIV was assessed as successful. NIV was successful in 3 out of 11 in-hospital non-survivors compared to 40 out of 67 survivors ( $27.3 \%$ vs $59.7 \%, p=0.045$ (pearson Ch-Square test). The survivor group had a significantly better tolerance to NIV compared to the non-survivors ( $80.6 \%$ vs. $12.5 \%$, $\mathrm{p}=0.000$ (pearson Chi-square test)). There was no significant difference in the percentage of patients with successful response between non-survivors and survivors after two years follow up (see Table S6.4 (see supplemental data)).

The univariate comparison between the survivors and non-survivors after 2-year follow-up are presented in Tables S6.3 and S6.4 (see supplemental data). Prior to NIV, several significant negative predictors were found: male gender, higher amount of pack years, prednisone maintenance therapy, atrial fibrillation, low $\mathrm{pH}$, change in medical policy restrictions. RR after 1 hour use of NIV was significantly higher in the nonsurvivors. The delta $\mathrm{PaCO}_{2}$ before start and at termination NIV was different but failed to reach statistical significance $(p=0.058)$.

In the multivariate analysis were entered the following clinically relevant or significant univariate analysis variables: advanced age, gender, $\mathrm{pH}$ before start NIV, RR $>20$ min, delta $\mathrm{paCO}_{2}$ before start NIV vs. at termination NIV, duration NIV (days), response (tolerance) to NIV. As independent factors associated with 2-year mortality advanced age, prolonged (>8 days) NIV use and the response (successful or not successful) to NIV assessed to be unsuccessful were identified (Table 6) (see main manuscript). Figure 6.3 (see main manuscript) show the Kaplan Meier survival curve of prolonged ( $>8$ days) NIV use (Logrank 0.025; Breslow 0.040) and unsuccessful NIV (Logrank 0.011; Breslow 0.002 ), and occurrence of readmission after discharge (Logrank 0.0001; Breslow $0.0001)$, which have negative impact on survival. 


\section{Tables and figures (extended version)}

Table S6.1 Characteristics of patients hospitalized for ECOPD with acute respiratory failure and requiring non-invasive ventilation at first, stratified by in-hospital mortality.

\begin{tabular}{|c|c|c|c|c|}
\hline & $\begin{array}{c}\text { All } \\
(n=78)\end{array}$ & $\begin{array}{c}\text { Hospital } \\
\text { survivors } \\
(n=67)\end{array}$ & $\begin{array}{c}\text { Hospital } \\
\text { deaths } \\
(n=11)\end{array}$ & p-value \\
\hline Male gender & $38(48.7)$ & $30(44.8)$ & $8(72.7)$ & 0.086 \\
\hline Age at admission, $y$ & $71.0 \pm 10.7$ & $69.9 \pm 10.8$ & $77.5 \pm 7.3$ & 0.027 \\
\hline \multicolumn{5}{|l|}{ Smoking status $(n=74)$} \\
\hline Non smoker & $1(1.3)$ & 0 & $1(11.1)$ & 0.001 \\
\hline Former smoker & 32 (41.0) & 25 (38.5) & $7(77.8)$ & \\
\hline Current smoker & $41(52.6)$ & $40(61.5)$ & 1 (11.1) & \\
\hline Pack years $(n=32 / 29 / 3)$ & $43.5 \pm 12.3$ & $42.5 \pm 12.1$ & $53.3 \pm 11.5$ & 0.148 \\
\hline \multicolumn{5}{|l|}{ GOLD stage } \\
\hline Gold II & $17(21.8)$ & $8(23.5)$ & $8(18.2)$ & \\
\hline Gold III & 21 (26.9) & $9(26.5)$ & $12(27.3)$ & \\
\hline Gold IV & 40 (51.3) & $16(47.1)$ & $24(54.5)$ & \\
\hline Post-BD FEV $1, \%$ pred & 39.0 (28.6-52.9) & $40.1(28.8-53.6)$ & $36.1(27.8-44.5)$ & 0.438 \\
\hline Post-BD FEV ${ }_{1}, \mathrm{~L}$ & $0.9 \pm 0.5$ & $1.0 \pm 0.5$ & $0.9 \pm 0.26$ & 0.455 \\
\hline $\mathrm{FEV}_{1} / \mathrm{FVC}$ & $38.3 \pm 11.2$ & $38.7 \pm 16.0$ & $35.4 \pm 9.3$ & 0.355 \\
\hline RV/TLC, \% (n=53/46/7) & $61.9 \pm 10.0$ & $61.6 \pm 10.5$ & $64.0 \pm 5.8$ & 0.568 \\
\hline TLCO/VA, \%pred (n=51/44/7) & $51.1 \pm 21.8$ & $51.2 \pm 22.6$ & $50.3 \pm 16.6$ & 0.927 \\
\hline Hospitalization for ECOPD previous year & $26(33.3)$ & $23(34.3)$ & $3(27.3)$ & 0.645 \\
\hline $0-1$ & 67 (85.9) & $58(86.6)$ & $9(81.8)$ & \\
\hline Frequent exacerbators $(>1)$ & $11(14.1)$ & $9(13.4)$ & $2(18.2)$ & 0.675 \\
\hline \multicolumn{5}{|l|}{ Living status $(\mathrm{n}=75)$} \\
\hline At home & 65 (83.3) & $56(86.2)$ & 10 (90.9) & 0.666 \\
\hline Alone & $10(12.8)$ & 10 (14.9) & 0 & \\
\hline Alone with care & $20(25.6)$ & $18(26.9)$ & $2(20.0)$ & \\
\hline Cohabitation & $10(12.8)$ & $9(13.4)$ & $1(10.0)$ & \\
\hline Cohabitation with care & $25(32.1)$ & $19(28.4)$ & $6(60.0)$ & 0.121 \\
\hline Resident (not at home) & $10(14.1)$ & $9(13.8)$ & $1(9.1)$ & 0.666 \\
\hline No additional care & $20(25.6)$ & $19(29.2)$ & $1(10.0)$ & 0.200 \\
\hline Treatment for COPD yes & 73 (93.6) & $62(92.5)$ & $11(100)$ & 0.645 \\
\hline SABA & $43(55.1)$ & $37(55.2)$ & $6(54.5)$ & 0.916 \\
\hline SAMA & 28 (35.9) & $23(34.3)$ & $5(45.5)$ & 0.740 \\
\hline LABA & $62(79.5)$ & $53(85.5)$ & $9(81.8)$ & 0.914 \\
\hline LAMA & 51 (65.4) & $43(64.2)$ & $8(72.7)$ & 0.814 \\
\hline ICS & $59(75.6)$ & 51 (76.1) & $8(72.7)$ & 0.871 \\
\hline Theophylline & $7(9.0)$ & $7(10.4)$ & 0 & 0.481 \\
\hline Prednisone $^{1}$ & $10(12.8)$ & $6(9.0)$ & $4(36.4)$ & 0.040 \\
\hline Antibiotic $^{1}$ & $4(5.1)$ & $2(3.0)$ & $2(18.2)$ & 0.100 \\
\hline LTOT & $30(38.5)$ & $25(37.3)$ & $5(45.5)$ & 0.820 \\
\hline
\end{tabular}


Table S6.1 (continued)

\begin{tabular}{|c|c|c|c|c|}
\hline & $\begin{array}{c}\text { All } \\
(n=78)\end{array}$ & $\begin{array}{c}\text { Hospital } \\
\text { survivors } \\
(n=67)\end{array}$ & $\begin{array}{c}\text { Hospital } \\
\text { deaths } \\
(n=11)\end{array}$ & p-value \\
\hline $\mathrm{CCl} .1$ & $2.0(1.0-3.0)$ & $2.0(1-3)$ & $3.0(2-4)$ & 0.041 \\
\hline CVD & $44(56.4)$ & $31(46.2)$ & $8(10.3)$ & 0.239 \\
\hline DM/metabolic syndrome & $22(28.2)$ & $19(24.4)$ & $3(3.8)$ & 0.941 \\
\hline Cancer disease & $13(16.7)$ & $9(11.5)$ & $4(5.1)$ & 0.059 \\
\hline $\mathrm{Cl}$ and Psych D & $20(26.6)$ & $19(24.4)$ & $1(1.3)$ & 0.175 \\
\hline Renal failure and UTD & $13(16.7)$ & $12(17.9)$ & $1(1.3)$ & 0.467 \\
\hline Infect and Immu comp & $14(17.9)$ & $10(12.8)$ & $4(5.1)$ & 0.086 \\
\hline Muscular and skeletal D & $24(30.8)$ & $22(28.2)$ & $2(2.6)$ & 0.329 \\
\hline GI-tract diseases & $25(32.1)$ & $18(23.1)$ & $7(9.0)$ & 0.015 \\
\hline
\end{tabular}

Categorical data are presented as $\mathrm{n}(\%)$, non-parametric data as median (IQR) and parametric data as mean \pm (SD). Chi- $\mathrm{X}^{2}$, Kruskal-wallis test and T-test were used for statistical analysis respectively. Abbrevations: PostBD: post bronchodilator; FEV1: forced expiratory volume in the first second; FVC: Forced expiratory vital capacity; TLC: Totoal lung capacity; RV: Residual volume; TLCO/VA: difusing capacity for carbon monxidedivided by alveolar volume; SABA: short-acting $\beta_{2}$-agonists; SAMA: short-acting anticholinergic; LABA: long-acting $\beta_{2}$-agonists; LAMA: long-acting anticholinergic; ICS: Inhaled glucocorticoids; LTOT: Longterm Oxygen therapy; $\mathrm{CCl}$ : Charlson Comorbidity Index. CVD: cardiovascular diseases; DM: diabetes mellitus: $\mathrm{Cl}$ : cognitive impairment; Psych D: psychological disturbances; UTD Urine tract diseases; D: diseases; Infect and Immu comp: (pulmonary) Infectious diseases or immune compromised; GI: gastro intestinal. ${ }^{1}$ preexisting maintenance treatment.

Table S6.2 Characteristics at emergency room presentation and non-invasive ventilation (NIV) results of patients hospitalized for ECOPD with acute respiratory failure and requiring non-invasive ventilation (NIV) at first, stratified by in-hospital mortality.

\begin{tabular}{|c|c|c|c|c|}
\hline Characteristics (n) & $\begin{array}{c}\text { All } \\
(n=78)\end{array}$ & $\begin{array}{c}\text { Hospital } \\
\text { survivors } \\
\text { (n=67) }\end{array}$ & $\begin{array}{c}\text { Hospital } \\
\text { deaths } \\
(n=11)\end{array}$ & p-value \\
\hline \multicolumn{5}{|l|}{ Vital signs } \\
\hline Temperature, ${ }^{\circ} \mathrm{C}(\mathrm{n}=72 / 61 / 11)$ & $37.0 \pm 0.93$ & $37.1 \pm 0.97$ & $37.0 \pm 0.7$ & 0.898 \\
\hline Temperature, $>38^{\circ} \mathrm{C}$ & $10(12.8)$ & $9(13.4)$ & $1(9.1)$ & 0.513 \\
\hline Systolic BP, mmHg ( $n=77)$ & $142.3 \pm 34.2$ & $143.1 \pm 34.4$ & $137.0 \pm 34.1$ & 0.585 \\
\hline Diastolic BP, mmHg ( $n=77)$ & $77.9 \pm 20.1$ & $79.4 \pm 20.1$ & $69.5 \pm 18.8$ & 0.132 \\
\hline Pulse, beats/min $(n=77)$ & 105 (95.0-117.5) & $105.0(93.8-116.3)$ & $105.0(95.0-120.0)$ & 0.021 \\
\hline Pulse, $>100 / \mathrm{min}$ & $43(55.1)$ & $36(53.7)$ & $7(63.6)$ & 0.784 \\
\hline $\mathrm{SpO}_{2}, \%(n=75)$ & $90.0(82-94)$ & $90.0(80.8-94.0)$ & $91.0(82.0-94.0)$ & 0.010 \\
\hline Additional oxygen $(n=73)$ & $44(60.3)$ & $28(43.8)$ & $5(45.5)$ & 0.280 \\
\hline \multicolumn{5}{|l|}{ Physical examination } \\
\hline Respiratory rate, /min $(n=42)$ & $30(24-34.3)$ & $30.0(23.5-33.0)$ & $31.4(24.5-39.0)$ & 0.683 \\
\hline Impaired consciousness ( $n=76)$ & $15(19.2)$ & $14(21.5)$ & $1(9.1)$ & 0.337 \\
\hline Peripheral edema ( $n=69)$ & 28 (35.9) & $26(38.8)$ & $2(18.2)$ & 0.337 \\
\hline Wheezing $(n=76)$ & $44(56.4)$ & $39(58.2)$ & $5(45.5)$ & 0.292 \\
\hline
\end{tabular}


Table S6.2 (continued)

\begin{tabular}{|c|c|c|c|c|}
\hline Characteristics (n) & $\begin{array}{c}\text { All } \\
(n=78)\end{array}$ & $\begin{array}{c}\text { Hospital } \\
\text { survivors } \\
(n=67)\end{array}$ & $\begin{array}{c}\text { Hospital } \\
\text { deaths } \\
(n=11)\end{array}$ & p-value \\
\hline \multicolumn{5}{|l|}{ Use accessory respiratory } \\
\hline Muscle $(n=26)$ & $17(21.8)$ & $16(23.9)$ & $1(9.1)$ & 0.483 \\
\hline Crackles $(n=75)$ & $32(41.0)$ & $29(43.3)$ & $3(27.3)$ & 0.433 \\
\hline Elevated CVP $(n=30)$ & $8(10.3)$ & $7(10.4)$ & $1(9.1)$ & 0.386 \\
\hline$B M I, k g / m^{2}(n=74)$ & $23.4 \pm 5.2$ & $23.9 \pm 5.4$ & $20.6 \pm 2.2$ & 0.002 \\
\hline \multicolumn{5}{|l|}{ Laboratory results } \\
\hline Hemoglobin, $\mathrm{mmol} / \mathrm{L}$ & $8.7 \pm 1.2$ & $8.6 \pm 1.1$ & $8.4 \pm 1.1$ & 0.500 \\
\hline$W B C, 10^{9} / L$ & $14.5 \pm 8.5$ & $13.4 \pm 5.8$ & $20.9 \pm 16.7$ & 0.168 \\
\hline $\mathrm{Na}, \mathrm{mmol} / \mathrm{L}$ & $137(134.0-139.3)$ & $137(134.0-140.0)$ & $136.0(134.0-137.0)$ & 0.968 \\
\hline Urea, $\mathrm{mmol} / \mathrm{L}(\mathrm{n}=76)$ & $7.4(5.0-10.3)$ & $7.0(4.8-10.0)$ & $10.0(7.7-16.0)$ & 0.037 \\
\hline Urea $\geq 8 \mathrm{mmol} / \mathrm{L}$ & $33(43.4)$ & $25(38.5)$ & $8(72.7)$ & 0.025 \\
\hline Creatinine, $\mathrm{mmol} / \mathrm{L}$ & 85 (76.8-90.5) & $84.0(63.0-105.0)$ & $106.0(81.0-137.0)$ & 0.082 \\
\hline Creatinine $>115 \mu \mathrm{mol} / \mathrm{L}$ & $18(23.1)$ & $13(19.4)$ & $5(45.5)$ & 0.057 \\
\hline$C R P, m g / L$ & $56.6(24.0-121.0)$ & $49.0(21.0-103.0)$ & $96.0(31.0-135.0)$ & 0.305 \\
\hline$C R P>50 \mathrm{mg} / \mathrm{l}$ & $40(51.3)$ & 33 (49.3) & $7(63.6)$ & 0.376 \\
\hline Glucose, $\mathrm{mmol} / \mathrm{L}(\mathrm{n}=74)$ & $9.4 \pm 4.7$ & $9.4 \pm 4.0$ & $9.6 \pm 7.6$ & 0.908 \\
\hline Troponine $\geq 0.01(n=38 / 32 / 6)$ & $17(44.7)$ & $12(37.5)$ & $5(83.3)$ & 0.038 \\
\hline \multicolumn{5}{|l|}{ Chest $X$-ray $(n=77)$} \\
\hline Consolidation & $25(32.1)$ & 20 (29.9) & $5(50.0)$ & 0.204 \\
\hline Signs of congestion & $12(15.4)$ & $10(14.9)$ & $2(20.0)$ & 0.680 \\
\hline \multicolumn{5}{|l|}{$E C G(n=67)$} \\
\hline Atrial fibrillation & $8(10.3)$ & $7(12.3)$ & $1(10.0)$ & 0.837 \\
\hline Signs of ischemia & $4(6.0)$ & $2(3.5)$ & $2(20.0)$ & 0.042 \\
\hline p-pulmonale & $13(16.7)$ & $11(19.3)$ & $2(20.0)$ & 0.959 \\
\hline \multicolumn{5}{|l|}{ ABG before NIV $(n=77)$} \\
\hline $\mathrm{pH}$ & $7.27(7.22-7.31)$ & $7.28(7.22-7.31)$ & $7.24(7.1-7.26)$ & 0.015 \\
\hline$<7.25$ & $28(36.4)$ & $21(31.8)$ & $7(63.6)$ & \\
\hline $7.25-7.35$ & $49(63.6)$ & $45(68.2)$ & $4(36.4)$ & 0.042 \\
\hline $\mathrm{PaCO}_{2}, \mathrm{kPa}$ & $10.0(8.5-11.2)$ & $10.0(8.5-11.0)$ & $10.8(8.3-12.1)$ & 0.322 \\
\hline $\mathrm{PaCO}_{2}>9.1 \mathrm{kPa}$ & $50(64.9)$ & $42(63.6)$ & $8(72.7)$ & 0.559 \\
\hline $\mathrm{HCO}_{3}{ }^{-} \geq 27.0 \mathrm{mmol} / \mathrm{L}$ & $61(79.2)$ & $56(84.8)$ & $5(54.5)$ & 0.003 \\
\hline $\mathrm{HCO}_{3}^{-}, \mathrm{mmol} / \mathrm{L}$ & $31.8(27.6-36.3)$ & $31.9(28.0-36.2)$ & $26.7(25.4-36.8)$ & 0.799 \\
\hline $\mathrm{BE}$ & $3.4(-1.0-6.6)$ & $3.7(-0.3-6.6)$ & $2.9(-5.4-6.6)$ & 0.256 \\
\hline$<-2.5$ & $14(18.2)$ & $9(13.6)$ & $5(45.5)$ & \\
\hline$-2.5-2.5$ & $21(27.3)$ & $21(31.8)$ & 0 & \\
\hline$>2.5$ & $42(54.5)$ & $36(54.5)$ & $6(54.5)$ & 0.013 \\
\hline $\mathrm{PaO}_{2}, \mathrm{kPa}$ & $8.6(7.2-10.7)$ & $8.6(7.4-10.5)$ & $9.6(6.3-12.3)$ & 0.799 \\
\hline $\mathrm{SaO}_{2}, \%$ & $89.0(84.0-93.0)$ & $89.0(84.0-92.0$ & $90.0(72.5-96.0)$ & 0.980 \\
\hline $\mathrm{SaO}_{2}<90 \%$ & 39 (54.9) & 34 (55.7) & $5(14.1)$ & 0.735 \\
\hline $\mathrm{PaO}_{2}<7.5 \mathrm{kPa}$ & $22(28.6)$ & $18(27.3)$ & $4(36.4)$ & 0.537 \\
\hline \multicolumn{5}{|l|}{ Policy restrictions } \\
\hline Assessed before admission & 14 (17.9) & 12 (17.9) & $2(18.2)$ & 0.983 \\
\hline Changed at admission & $36(46.2)$ & $26(38.8)$ & 10 (90.9) & 0.001 \\
\hline Present before NIV & $46(59.0)$ & $36(53.7)$ & 10 (90.9) & 0.020 \\
\hline
\end{tabular}


Table S6.2 (continued)

\begin{tabular}{|c|c|c|c|c|}
\hline Characteristics (n) & $\begin{array}{c}\text { All } \\
(n=78)\end{array}$ & $\begin{array}{c}\text { Hospital } \\
\text { survivors } \\
\text { (n=67) }\end{array}$ & $\begin{array}{c}\text { Hospital } \\
\text { deaths } \\
(n=11)\end{array}$ & p-value \\
\hline \multicolumn{5}{|l|}{ Clinical data } \\
\hline LOS, days & $16.5(11.0-28.25)$ & $17.0(11.0-27.0)$ & $11.0(3.0-31.0)$ & 0.438 \\
\hline NIV, days & $5.0(2.8-7.0)$ & $5.0(2.0-7.0)$ & $7.0(3.0-11.0)$ & 0.337 \\
\hline NIV $\leq 8$ days & $63(85.9)$ & $56(83.6)$ & $7(63.6)$ & 0.120 \\
\hline IPAP, $\mathrm{cm} \mathrm{H}_{2} \mathrm{O}$ & $20.8 \pm 4.3$ & $20.5 \pm 4.3$ & $22.9 \pm 3.8$ & 0.081 \\
\hline $\mathrm{EPAP}, \mathrm{cm} \mathrm{H} \mathrm{H}_{2} \mathrm{O}$ & $6.4 \pm 1.5$ & $6.2 \pm 1.5$ & $7.1 \pm 1.4$ & 0.087 \\
\hline \multicolumn{5}{|l|}{ NIV response after $1 \mathrm{~h}$} \\
\hline Delta $\mathrm{pCO}_{2}$ before NIV-1h, $\mathrm{kPa}$ & $1.4 \pm 1.2$ & $1.4 \pm 1.3$ & $1.6 \pm 1.1$ & 0.548 \\
\hline $\mathrm{pH} \geq 7.35$ at $1 \mathrm{~h}$ & $22(28.6)$ & $21(31.8)$ & $1(9.1)$ & 0.122 \\
\hline $\mathrm{RR}$ at $1 \mathrm{~h}, / \mathrm{min}$ & $22.8 \pm 5.9$ & $22.2 \pm 5.9$ & $26.7 \pm 4.4$ & 0.034 \\
\hline $\mathrm{RR}>20 / \mathrm{min}$ at $1 \mathrm{~h}$ & 39 (61.9) & $31(57.4)$ & $8(88.9)$ & 0.072 \\
\hline \multicolumn{5}{|l|}{ NIV response after $24 \mathrm{~h}$} \\
\hline Delta $\mathrm{PaCO}_{2}$ before NIV-24h, kPa & $2.1 \pm 2.2$ & $2.1 \pm 2.3$ & $2.2 \pm 1.9$ & 0.888 \\
\hline $\mathrm{pH} \geq 7.3524 \mathrm{~h}(\mathrm{n}=63)$ & $35(55.6)$ & $32(60.4)$ & $3(30.0)$ & 0.076 \\
\hline $\mathrm{RR} 24 \mathrm{~h}, / \mathrm{min}(\mathrm{n}=56)$ & $21.2 \pm 4.5$ & $21.4 \pm 4.6$ & $19.9 \pm 3.6$ & 0.401 \\
\hline $\mathrm{RR}>20 / \mathrm{min}$ at $24 \mathrm{~h}$ & $25(44.6)$ & $22(44.9)$ & $3(42.9)$ & 0.919 \\
\hline \multicolumn{5}{|l|}{ NIV response at stop NIV } \\
\hline Delta $\mathrm{PaCO}_{2}$ before NIV-stop, kPa & $2.3 \pm 2.9$ & $2.4 \pm 2.2$ & $1.8 \pm 3.4$ & 0.519 \\
\hline $\begin{array}{l}\text { Delta bicarbonate before NIV-stop, } \\
\mathrm{mmol} / \mathrm{l}\end{array}$ & $-5.2 \pm-0.9$ & $-6.0 \pm 42.0$ & $0.4 \pm 7.2$ & 0.631 \\
\hline BE at stop NIV & $7.6 \pm 13.3$ & $8.6 \pm 13.8$ & $1.1 \pm 5.6$ & 0.099 \\
\hline Delta BE before NIV-stop( $n=74)$ & $5.0 \pm 13.0$ & $5.8 \pm 13.5$ & $0.1 \pm 8.7$ & 0.201 \\
\hline Successful NIV & $43(55.1)$ & $40(59.7)$ & $3(27.3)$ & 0.045 \\
\hline Tolerance NIV & $55(73.3)$ & $54(98.2)$ & $1(1.8)$ & 0.000 \\
\hline
\end{tabular}

Categorical data are presented as $\mathrm{n}(\%)$, non-parametric data as median (IQR) and parametric data as mean \pm (SD). Chi- $\mathrm{X}^{2}$, Kruskal-wallis test and T-test were used for statistical analysis respectively. Abbrevations: BP: blood pressure; CVP: central venous pressure; BMI: body mass index; NIV: non-invasive ventilation; WBC: white blood cell count, Na: Sodium; ECG: electrocardiogram; ABG: arterial blood gas; LOS: Length of hospital stay; IPAP: inspiratory positive air pressure; EPAP: Expiratory positive air pressure; RR: respiratory rate; BE: base excess; NIV: non-invasive ventilation. 
Table S6.3 Characteristics of patients hospitalized for ECOPD with acute respiratory failure and requiring non-invasive ventilation at first, stratified by 2-year outcome.

\begin{tabular}{|c|c|c|c|c|}
\hline & $\begin{array}{c}\text { All } \\
(n=78)\end{array}$ & $\begin{array}{c}\text { Long-term } \\
\text { survivors }(n=34)\end{array}$ & $\begin{array}{c}\text { Long-term } \\
\text { Deaths }(n=44)\end{array}$ & p-value \\
\hline Male gender & $38(48.7)$ & $11(28.9)$ & $27(71.1)$ & 0.011 \\
\hline Age at admission, y & $71.0 \pm 10.7$ & $66.9 \pm 11.6$ & $74.1 \pm 8.9$ & 0.003 \\
\hline \multicolumn{5}{|l|}{ Smoking status } \\
\hline Non smoker & $1(1.3)$ & 0 & $1(1.3)$ & 0.176 \\
\hline Former smoker & $32(41.0)$ & $13(38.2)$ & $19(43.2)$ & \\
\hline Current smoker & $41(52.6)$ & $21(61.8)$ & $20(45.5)$ & \\
\hline Unknown & $4(5.1)$ & 0 & $4(9.1)$ & \\
\hline Pack years $(n=32 ; 18 ; 14)$ & $43.5 \pm 12.3$ & $39.2 \pm 12.4$ & $48.9 \pm 10.2$ & 0.024 \\
\hline \multicolumn{5}{|l|}{ GOLD stage } \\
\hline Gold II & $16(20.5)$ & $8(23.5)$ & $8(18.2)$ & \\
\hline Gold III & 21 (26.9) & $9(26.5)$ & $12(27.3)$ & \\
\hline Gold IV & $40(51.3)$ & $16(47.1)$ & $24(54.5)$ & 0.620 \\
\hline Post-BD FEV $1, \%$ pred & $39.0(28.6-52.9)$ & $40.2(29.9-54.3)$ & $36.9(27.2-49.8)$ & 0.351 \\
\hline Post-BD FEV ${ }_{1}, L$ & $0.9 \pm 0.5$ & $0.9 \pm 0.5$ & $1.0 \pm 0.46$ & 0.908 \\
\hline$F E V_{1} / F V C$ & $38.3 \pm 11.2$ & $39.4 \pm 12.1$ & $37.4 \pm 10.4$ & 0.417 \\
\hline$R V / T L C, \%(n=53 ; 23 ; 30)$ & $61.9 \pm 10.0$ & $59.4 \pm 11.8$ & $63.9 \pm 8.1$ & 0.105 \\
\hline TLCO/VA, \%pred $(n=51 ; 23 ; 28)$ & $51.1 \pm 21.8$ & $54.6 \pm 25.0$ & $48.2 \pm 18.7$ & 0.305 \\
\hline Hospitalization for ECOPD previous first year & $26(33.3)$ & $8(23.5)$ & $18(56.4)$ & 0.106 \\
\hline $0-1$ & $67(85.9)$ & $30(44.8)$ & $37(55.2)$ & \\
\hline Frequent exacerbators $(>1)$ & $11(14.1)$ & $4(11.8)$ & $7(15.9)$ & 0.602 \\
\hline \multicolumn{5}{|l|}{ Living status $n=75$} \\
\hline At Home & $65(83.3)$ & $30(88.2)$ & $36(85.7)$ & 0.746 \\
\hline Alone & $10(12.8)$ & $5(14.7)$ & $5(11.6)$ & \\
\hline Alone with care & $20(25.6)$ & $11(32.4)$ & 9 (20.9) & \\
\hline Cohabitation & $10(12.8)$ & $7(20.6)$ & $3(7.0)$ & \\
\hline Cohabitation with care & $25(32.1)$ & $7(20.6)$ & $18(41.9)$ & 0.285 \\
\hline Resident (not at home) & $10(14.1)$ & $4(11.8)$ & $6(14.3)$ & 0.746 \\
\hline No additional care & $20(25.6)$ & $12(35.3)$ & $8(19.5)$ & 0.124 \\
\hline Treatment for COPD yes & 73 (93.6) & $30(88.2)$ & 43 (97.7) & 0.214 \\
\hline$S A B A$ & $43(55.1)$ & $20(58.8)$ & $23(52.3)$ & 0.399 \\
\hline SAMA & 28 (35.9) & $14(41.2)$ & $14(31.8)$ & 0.329 \\
\hline$\angle A B A$ & $62(79.5)$ & $23(67.6)$ & $39(88.6)$ & 0.061 \\
\hline LAMA & $51(65.4)$ & $17(50.0)$ & $34(77.3)$ & 0.032 \\
\hline ICS & $59(75.6)$ & $25(73.5)$ & 34 (77.3) & 0.513 \\
\hline Theophylline & $7(9.0)$ & $4(11.8)$ & $3(6.8)$ & 0.377 \\
\hline Prednisone $e^{1}$ & $10(12.8)$ & $1(2.9)$ & $9(20.5)$ & 0.04 \\
\hline Antibiotics $^{1}$ & $4(5.1)$ & 0 & $4(9.1)$ & 0.107 \\
\hline LTOT & $30(38.5)$ & $10(29.4)$ & $20(45.5)$ & 0.210 \\
\hline $\mathrm{CCl}$ & $2(1-3)$ & $2(1-3)$ & $2(1-3)$ & 0.478 \\
\hline CVD & $44(56.4)$ & $17(21.8)$ & $27(34.6)$ & 0.316 \\
\hline DM/metabolic syndrome & $22(28.2)$ & $10(29.4)$ & $12(15.4)$ & 0.835 \\
\hline Cancer disease & $13(16.7)$ & $4(5.1)$ & $9(11.5)$ & 0.307 \\
\hline $\mathrm{Cl}$ and Psych D & $20(25.6)$ & $10(12.8)$ & $10(12.8)$ & 0.503 \\
\hline Renal failure and UTD & $13(16.7)$ & $7(9.0)$ & $6(7.7)$ & 0.414 \\
\hline Infect or immu comp & 14 (17.9) & $4(5.1)$ & $10(12.8)$ & 0.211 \\
\hline Muscular and skeletal D & $24(30.8)$ & $9(11.5)$ & $15(19.2)$ & 0.470 \\
\hline Gl-tract diseases & $25(32.1)$ & $5(6.4)$ & $20(26.6)$ & 0.004 \\
\hline
\end{tabular}


Categorical data are presented as $\mathrm{n}(\%)$, non-parametric data as median (IQR) and parametric data as mean \pm (SD). Chi- $\mathrm{X}^{2}$, Kruskal-wallis test and T-test were used for statistical analysis respectively. Abbrevations: : PostBD: post bronchodilator; FEV1: forced expiratory volume in the first second; FVC: Forced expiratory vital capacity; TLC: Totoal lung capacity; RV: Residual volume; TLCO/VA: difusing capacity for carbon monxidedivided by alveolar volume; SABA: short-acting $\beta_{2}$-agonists; SAMA: short-acting anticholinergic; LABA: long-acting $\beta_{2}$-agonists; LAMA: long-acting anticholinergic; ICS: Inhaled glucocorticoids; LTOT: Longterm Oxygen therapy; $\mathrm{CCl}$ : Charlson Comorbidity Index. CVD: cardiovascular diseases; DM: diabetes mellitus: $\mathrm{Cl}$ : cognitive impairment; Psych D: psychological disturbances; UTD Urine tract diseases; D: diseases; Infect and Immu comp: (pulmonary) Infectious diseases or immune compromised; GI gastro intestinal. ${ }^{1}$ pre-existing maintenance treatment.

Table S6.4 Characteristics at emergency room presentation and non-invasive ventilation (NIV) results of patients hospitalized for ECOPD with acute respiratory failure and requiring non-invasive ventilation at first, stratified by 2-year outcome.

\begin{tabular}{|c|c|c|c|c|}
\hline Characteristics (n) & $\begin{array}{c}\text { All } \\
(n=78)\end{array}$ & $\begin{array}{l}\text { Long-term } \\
\text { survivors } \\
(n=34)\end{array}$ & $\begin{array}{c}\text { Long-term } \\
\text { deaths } \\
(n=44)\end{array}$ & p-value \\
\hline \multicolumn{5}{|l|}{ Vital signs } \\
\hline Temperature, ${ }^{\circ} \mathrm{C}(\mathrm{n}=72 / 31 / 41)$ & $37.1(36.5-37.5)$ & $37.1 \pm 1.0$ & $36.9 \pm 0.8$ & 0.194 \\
\hline Temperature $>38^{\circ} \mathrm{C}$ & $10(12.8)$ & $6(17.6)$ & $4(9.1)$ & 0.481 \\
\hline Systolic BP, mmHg ( $n=77)$ & $142.3 \pm 34.2$ & $128.1 \pm 29.0$ & $153.4 \pm 33.5$ & 0.001 \\
\hline Diastolic BP, mmHg ( $n=77)$ & $77.9 \pm 20.1$ & $73.4 \pm 14.9$ & $81.5 \pm 23.0$ & 0.066 \\
\hline Pulse, beats/min $(n=77)$ & 105 (95.0-117.5) & $95.0(84.8-115.0)$ & $110.0(95.0-120.0)$ & 0.116 \\
\hline Pulse $>100 / \mathrm{min}$ & $43(55.1)$ & $16(47.1)$ & $27(61.4)$ & 0.260 \\
\hline $\mathrm{SpO}_{2}, \%(\mathrm{n}=75)$ & $90.0(82-94)$ & $91.0(83.5-94.8)$ & $90.0(80.0-94.0)$ & 0.443 \\
\hline Additional oxygen $(n=73)$ & $44(60.3)$ & $18(58.1)$ & $26(61.9)$ & 0.497 \\
\hline \multicolumn{5}{|l|}{ Physical examination } \\
\hline Respiratory rate/min $(n=42)$ & $30.0(24-34.3)$ & $25.0(22.5-30.0)$ & $30.0(27.5-35.0)$ & 0.26 \\
\hline Impaired consciousness ( $n=76)$ & $15(19.2)$ & $5(14.7)$ & $10(22.7)$ & 0.383 \\
\hline Peripheral edema $(n=69)$ & $28(35.9)$ & $12(35.3)$ & $16(36.4)$ & 0.345 \\
\hline Wheezing ( $n=76)$ & $44(56.4)$ & $21(61.8)$ & $23(52.3)$ & 0.663 \\
\hline Use accessory respiratory muscle $(n=26)$ & $17(21.8)$ & $10(29.4)$ & 7 (15.9) & 0.204 \\
\hline Crackles $(n=75)$ & $32(41.0)$ & $14(41.2)$ & $18(40.9)$ & 0.935 \\
\hline Elevated CVP $(n=30)$ & $8(10.3)$ & $4(11.8)$ & $4(9.1)$ & 0.888 \\
\hline Body mass index, $\mathrm{kg} / \mathrm{m}^{2}$ & $23.4 \pm 5.2$ & $24.8 \pm 5.6$ & $22.3 \pm 4.7$ & 0.41 \\
\hline \multicolumn{5}{|l|}{ Chest X-ray $(n=77)$} \\
\hline Consolidation & $25(32.1)$ & $8(23.5)$ & $17(39.5)$ & 0.136 \\
\hline Signs of congestion & $12(15.4)$ & $4(11.8)$ & $8(18.6)$ & 0.411 \\
\hline \multicolumn{5}{|l|}{$\operatorname{ECG}(n=67)$} \\
\hline Atrial fibrillation & $8(10.3)$ & $1(3.4)$ & $7(18.4)$ & 0.061 \\
\hline Signs of ischemia & $4(5.1)$ & $1(3.4)$ & $4(7.9)$ & 0.447 \\
\hline p-Pulmonary & $13(16.7)$ & $6(20.7)$ & 7 (18.4) & 0.816 \\
\hline
\end{tabular}


Table S6.4 (continued)

\begin{tabular}{|c|c|c|c|c|}
\hline Characteristics (n) & $\begin{array}{c}\text { All } \\
(n=78)\end{array}$ & $\begin{array}{c}\text { Long-term } \\
\text { survivors } \\
(\mathrm{n}=34)\end{array}$ & $\begin{array}{c}\text { Long-term } \\
\text { deaths } \\
(n=44)\end{array}$ & p-value \\
\hline \multicolumn{5}{|l|}{ ABG before start NIV $(n=77)$} \\
\hline $\mathrm{pH}$ & 7.27 (7.22-7.31) & $7.28(7.23-7.31)$ & 7.25 (7.18-7.29) & 0.024 \\
\hline$<7.25$ & $28(36.4)$ & $8(23.5)$ & $20(46.5)$ & \\
\hline $7.25-7.35$ & $49(63.6)$ & $26(76.5)$ & $23(53.5)$ & 0.037 \\
\hline $\mathrm{PaCO}_{2}, \mathrm{kPa}$ & $10.0(8.5-11.2)$ & $9.78 .5-10.5)$ & $10.3(8.6-11.5)$ & 0.270 \\
\hline $\mathrm{PaCO}_{2}>9.1 \mathrm{kPa}$ & $50(64.9)$ & $20(58.8)$ & $30(69.8)$ & 0.318 \\
\hline $\mathrm{HCO}_{3}{ }^{-} \geq 27,0 \mathrm{mmol} / \mathrm{L}$ & $61(79.2)$ & $30(88.2)$ & $31(72.1)$ & 0.083 \\
\hline $\mathrm{HCO}_{3}^{-}, \mathrm{mmol} / \mathrm{L}$ & $31.8(27.6-36.3)$ & $31.4(27.8-73.0)$ & $31.8(26.7-36.0)$ & 0.538 \\
\hline Base excess & $3.4(-1.0-6.6)$ & $3.9(-0.3-7.0)$ & $3.1(-2.6-6.5)$ & 0.340 \\
\hline$<-2,5$ & $14(18.2)$ & $3(8.8)$ & $11(25.6)$ & \\
\hline$-2,5-2,5$ & $21(27.3)$ & $12(35.3)$ & 9 (20.9) & \\
\hline$>2,5$ & $42(54.5)$ & 19 (55.9) & $23(53.5)$ & 0.111 \\
\hline $\mathrm{PaO}_{2}, \mathrm{kPa}$ & $8.6(7.2-10.7)$ & $8.5(7.2-10.5)$ & $8.8(7.0-10.7)$ & 0.955 \\
\hline $\mathrm{SaO}_{2}, \%$ & $89.0(84.0-93.0)$ & $89.0(85.0-93.0)$ & $88.0(80.0-93.0)$ & 0.260 \\
\hline $\mathrm{SaO}_{2}<90 \%$ & 39 (54.9) & $16(51.6)$ & $23(57.5)$ & 0.621 \\
\hline $\mathrm{PaO}_{2}<7.5 \mathrm{kPa}$ & $22(28.6)$ & $11(32.4)$ & $11(25.6)$ & 0.514 \\
\hline \multicolumn{5}{|l|}{ Laboratory results } \\
\hline Hemoglobin mmol/L & $8.7 \pm 1.2$ & $8.9 \pm 0.9$ & $8.6 \pm 1.3$ & 0.244 \\
\hline WBC $10^{9} / L(362 / 331 / 31)$ & $14.5 \pm 8.5$ & $13.2 \pm 5.8$ & $15.4 \pm 10.1$ & 0.264 \\
\hline $\mathrm{Na} \mathrm{mmol/L}$ & 137 (134-139.3) & 137 (134.0-139.0) & 137.0(134.0-140.0) & 0.907 \\
\hline Urea $\mathrm{mmol} / \mathrm{L}(\mathrm{n}=76)$ & $7.4(5.0-10.3)$ & $7.3(4.3-9.7)$ & $7.7(6.2-10.6)$ & 0.314 \\
\hline Creatinine $\mathrm{mmol} / \mathrm{L}$ & $85(76.8-90.5)$ & $85(66.5-107.0)$ & $84.5(63.5-114.0)$ & 0.892 \\
\hline Creatinine $>115 \mu \mathrm{mol} / \mathrm{L}$ & $18(23.1)$ & $7(20.6)$ & $11(25.0)$ & 0.647 \\
\hline CRP mg/L & $56.6(24.0-121.0)$ & $75.0(11.5-153.0)$ & $46.0(25.5-94.0)$ & 0.268 \\
\hline$C R P>50 \mathrm{mg} / \mathrm{l}$ & $40(51.3)$ & $19(55.9)$ & $21(47.7)$ & 0.475 \\
\hline Glucose mmol/L $(n=74)$ & $9.4 \pm 4.7$ & $7.8(6.4-10.3)$ & $7.6(6.6-11.3)$ & 0.756 \\
\hline \multicolumn{5}{|l|}{ Limited policy restrictions } \\
\hline Assessed before admission & 14 (17.9) & $5(14.7)$ & $9(20.5)$ & 0.512 \\
\hline changed at admission & $36(46.2)$ & $8(23.5)$ & $28(63.6)$ & 0.000 \\
\hline present before NIV & $46(59.0)$ & $13(38.2)$ & $33(75.0)$ & 0.001 \\
\hline \multicolumn{5}{|l|}{ Clinical data } \\
\hline LOS, days & $16.5(11.0-28.25)$ & $13.5(10.0-18.3)$ & $17.0(8.3-28.0)$ & 0.528 \\
\hline NIV, days & $5.0(2.8-7.0)$ & $4.0(3.0-6.0)$ & $5.0(2.0-10.8)$ & 0.248 \\
\hline NIV $>8$ days & $15(19.2)$ & $3(8.8)$ & $12(27.3)$ & 0.040 \\
\hline IPAP, $\mathrm{cm} \mathrm{H}_{2} \mathrm{O}$ & $20.8 \pm 4.3$ & $21.2 \pm 3.9$ & $20.6 \pm 4.5$ & 0.535 \\
\hline $\mathrm{EPAP}, \mathrm{cm} \mathrm{H} \mathrm{H}_{2} \mathrm{O}$ & $6.4 \pm 1.5$ & $6.3 \pm 1.3$ & $6.4 \pm 1.7$ & 0.744 \\
\hline
\end{tabular}


Table S6.4 (continued)

\begin{tabular}{|c|c|c|c|c|}
\hline Characteristics (n) & $\begin{array}{c}\text { All } \\
(n=78)\end{array}$ & $\begin{array}{l}\text { Long-term } \\
\text { survivors } \\
(n=34)\end{array}$ & $\begin{array}{l}\text { Long-term } \\
\text { deaths } \\
(n=44)\end{array}$ & p-value \\
\hline \multicolumn{5}{|l|}{ NIV response after $1 \mathrm{~h}$} \\
\hline Delta $\mathrm{PaCO}_{2}$ before NIV-1h, kPa & $1.4 \pm 1.2$ & $1.1 \pm 1.1$ & $1.6 \pm 1.3$ & 0.094 \\
\hline $\mathrm{pH} \geq 7.35$ at1h & $22(28.6)$ & $12(35.3)$ & $10(23.3)$ & 0.246 \\
\hline $\mathrm{RR}$ at $1 \mathrm{~h}, / \mathrm{min}$ & $22.8 \pm 5.9$ & $21.0 \pm 6.1$ & $24.3 \pm 5.4$ & 0.027 \\
\hline $\mathrm{RR}>20 / \mathrm{min}$ at $1 \mathrm{~h}$ & 39 (61.9) & $14(48.3)$ & $25(73.5)$ & 0.040 \\
\hline \multicolumn{5}{|l|}{ NIV response after $24 \mathrm{~h}$} \\
\hline Delta $\mathrm{pCO}_{2}$ before NIV-24h, $\mathrm{kPa}$ & $2.1 \pm 2.2$ & $2.0 \pm 1.9$ & $2.3 \pm 2.4$ & 0.669 \\
\hline $\mathrm{pH} \geq 7.3524 \mathrm{~h}(\mathrm{n}=63)$ & $35(55.6)$ & $16(59.3)$ & $19(52.8)$ & 0.608 \\
\hline $\mathrm{RR} 24 \mathrm{~h}, / \mathrm{min}(\mathrm{n}=56)$ & $21.2 \pm 4.5$ & $20.4 \pm 3.7$ & $21.9 \pm 5.1$ & 0.204 \\
\hline $\mathrm{RR}>20 / \mathrm{min}$ at $24 \mathrm{~h}$ & $25(44.6)$ & $9(34.6)$ & $16(53.3)$ & 0.160 \\
\hline \multicolumn{5}{|l|}{ NIV response at stop NIV } \\
\hline Delta before NIV-stop $\mathrm{PaCO}_{2}, \mathrm{kPa}$ & $2.3 \pm 2.9$ & $2.6 \pm 17.5$ & $-2.8 \pm 2.9$ & 0.058 \\
\hline $\begin{array}{l}\text { Delta bicarbonate before NIV-stop, } \\
\mathrm{mmol} / \mathrm{l}\end{array}$ & $-5.2 \pm-0.9$ & $-10.8 \pm 57.5$ & $-0.3 \pm 5.4$ & 0.252 \\
\hline BE at stop NIV & $7.6 \pm 13.3$ & $10.7 \pm 18.6$ & $5.0 \pm 5.2$ & 0.067 \\
\hline Delta BE before NIV-stop $(n=74)$ & $5.0 \pm 13.0$ & $7.3 \pm 18.0$ & $6.2 \pm 5.9$ & 0.180 \\
\hline Successful NIV & $43(55.1)$ & $20(58.8)$ & $23(52.3)$ & 0.564 \\
\hline Tolerance/ adherence NIV & $55(73.3)$ & $28(82.4)$ & 27 (65.9) & 0.108 \\
\hline
\end{tabular}

Categorical data are presented as $\mathrm{n}(\%)$, non-parametric data as median (IQR) and parametric data as mean \pm (SD). Chi- $\mathrm{X}^{2}$, Kruskal-wallis test and T-test were used for statistical analysis respectively. Abbrevations: ABG: arterial blood gas; LOS: Length of hospital stay; IPAP inspiratory positive air pressure; EPAP: Expiratory positive air pressure; RR: respiratory rate; BE: base excess; NIV: non-invasive ventilation.

Table S6.5 Readmission data in study population during two years follow-up.

\begin{tabular}{lc}
\hline Readmission data & $\mathbf{n = 6 7}$ \\
\hline Total number readmissions $<2$ years & 83 (times) \\
1 readmission $<2$ years & $10(14.9)$ \\
2 readmissions $<2$ years & $10(14.9)$ \\
3 or more readmissions $<2$ years & $12(17.9)$ \\
H-ECOPD free-interval days & $124.5(32.8-239.8)$ \\
Combined dead or H-ECOPD <1year & $39(58.2)$ \\
Combined dead or H-ECOPD <2 years & $44(65.7)$ \\
Readmission NIV needed $<2$ years & $38($ times) \\
1 & $10(14.9)$ \\
2 or more & $10(14.9)$ \\
Readmission IV needed $<2$ years (once) & $6(9.0)$ \\
\hline
\end{tabular}

Data presented as $\mathrm{n}(\%)$ or abnormal distribution median (IQR). Abbrevations: H-ECOPD: Hospitalised exacerbation of COPD; NIV: non-invasive mechanical ventilation: IV: invasive mechanical ventilation. 


\section{References}

1. Foo, J., et al., Continuing to Confront COPD International Patient Survey: Economic Impact of COPD in 12 Countries. PLoS One, 2016. 11(4): p. e0152618.

2. Dransfield, M.T., et al., Acute Exacerbations and Lung Function Loss in Smokers with and without Chronic Obstructive Pulmonary Disease. Am J Respir Crit Care Med, 2017. 195(3): p. 324-330.

3. Soler-Cataluna, J.J., et al., Severe acute exacerbations and mortality in patients with chronic obstructive pulmonary disease. Thorax, 2005. 60(11): p. 925-31.

4. Slenter, R.H., et al., Predictors of 1-year mortality at hospital admission for acute exacerbations of chronic obstructive pulmonary disease. Respiration, 2013. 85(1): p. 15-26.

5. Connors, A.F., Jr., et al., Outcomes following acute exacerbation of severe chronic obstructive lung disease. The SUPPORT investigators (Study to Understand Prognoses and Preferences for Outcomes and Risks of Treatments). Am J Respir Crit Care Med, 1996. 154(4 Pt 1): p. 959-67.

6. Groenewegen, K.H., A.M. Schols, and E.F. Wouters, Mortality and mortality-related factors after hospitalization for acute exacerbation of COPD. Chest, 2003. 124(2): p. 459-67.

7. Steer, J., J. Gibson, and S.C. Bourke, The DECAF Score: predicting hospital mortality in exacerbations of chronic obstructive pulmonary disease. Thorax, 2012. 67(11): p. 970-6.

8. Sprooten, R.T.M., et al., Risk stratification for short-term mortality at hospital admission for acute exacerbations of COPD. Respirology, 2019.

9. Santibanez, M., et al., Predictors of Hospitalized Exacerbations and Mortality in Chronic Obstructive Pulmonary Disease. PLoS One, 2016. 11(6): p. e0158727.

10. Wedzicha, J.A.E.C.-C., et al., Management of COPD exacerbations: a European Respiratory Society/American Thoracic Society guideline. Eur Respir J, 2017. 49(3).

11. Chandra, D., et al., Outcomes of noninvasive ventilation for acute exacerbations of chronic obstructive pulmonary disease in the United States, 1998-2008. Am J Respir Crit Care Med, 2012. 185(2): p. 152-9.

12. Titlestad, I.L., A.T. Lassen, and J. Vestbo, Long-term survival for COPD patients receiving noninvasive ventilation for acute respiratory failure. Int J Chron Obstruct Pulmon Dis, 2013. 8: p. 215-9.

13. Lindenauer, P.K., et al., Outcomes associated with invasive and noninvasive ventilation among patients hospitalized with exacerbations of chronic obstructive pulmonary disease. JAMA Intern Med, 2014. 174(12): p. 1982-93.

14. Confalonieri, M., et al., Noninvasive mechanical ventilation improves the immediate and long-term outcome of COPD patients with acute respiratory failure. Eur Respir J, 1996. 9(3): p. 422-30.

15. Confalonieri, M., et al., A chart of failure risk for noninvasive ventilation in patients with COPD exacerbation. Eur Respir J, 2005. 25(2): p. 348-55.

16. Tokgoz Akyil, F., et al., Patient Outcome after Chronic Obstructive Pulmonary Disease Exacerbations Requiring Non-invasive Ventilation during Hospitalization. Arch Bronconeumol, 2016. 52(9): p. 470-6.

17. Chung, L.P., et al., Five-year outcome in COPD patients after their first episode of acute exacerbation treated with non-invasive ventilation. Respirology, 2010. 15(7): p. 1084-91.

18. Lindenauer, P.K., et al., Risk Trajectories of Readmission and Death in the First Year after Hospitalization for Chronic Obstructive Pulmonary Disease. Am J Respir Crit Care Med, 2018. 197(8): p. 1009-1017.

19. Sitges, M., et al., Interaliasing distance of the flow convergence surface for determining mitral regurgitant volume: a validation study in a chronic animal model. J Am Coll Cardiol, 2001. 38(4): p. 1195202.

20. Davidson, A.C., et al., BTS/ICS guideline for the ventilatory management of acute hypercapnic respiratory failure in adults. Thorax, 2016. 71 Suppl 2: p. ii1-35.

21. Nicolini, A., et al., Noninvasive ventilation for hypercapnic exacerbation of chronic obstructive pulmonary disease: factors related to noninvasive ventilation failure. Pol Arch Med Wewn, 2014. 124(10): p. 525-31.

22. Plant, P.K., J.L. Owen, and M.W. Elliott, Non-invasive ventilation in acute exacerbations of chronic obstructive pulmonary disease: long term survival and predictors of in-hospital outcome. Thorax, 2001. 56(9): p. 708-12. 
23. Martinez-Urbistondo, D., et al., Mortality Prediction in Patients Undergoing Non-Invasive Ventilation in Intermediate Care. PLoS One, 2015. 10(10): p. e0139702.

24. Sainaghi, P.P., et al., Natural history and risk stratification of patients undergoing non-invasive ventilation in a non-ICU setting for severe COPD exacerbations. Intern Emerg Med, 2016. 11(7): p. 102930.

25. Ucgun, I., et al., Predictors of hospital outcome and intubation in COPD patients admitted to the respiratory ICU for acute hypercapnic respiratory failure. Respir Med, 2006. 100(1): p. 66-74.

26. Steriade, A.T., et al., Predictors of outcome of noninvasive ventilation in severe COPD exacerbation. BMC Pulm Med, 2019. 19(1): p. 131.

27. Gadre, S.K., et al., Acute respiratory failure requiring mechanical ventilation in severe chronic obstructive pulmonary disease (COPD). Medicine (Baltimore), 2018. 97(17): p. e0487.

28. Bott, J., et al., Randomised controlled trial of nasal ventilation in acute ventilatory failure due to chronic obstructive airways disease. Lancet, 1993. 341(8860): p. 1555-7.

29. Vogelmeier, C.F., et al., Global Strategy for the Diagnosis, Management, and Prevention of Chronic Obstructive Lung Disease 2017 Report: GOLD Executive Summary. Eur Respir J, 2017. 49(3).

30. Penuelas, O., et al., Characteristics and outcomes of ventilated patients according to time to liberation from mechanical ventilation. Am J Respir Crit Care Med, 2011. 184(4): p. 430-7.

31. Martin-Gonzalez, F., et al., Effectiveness and predictors of failure of noninvasive mechanical ventilation in acute respiratory failure. Med Intensiva, 2016. 40(1): p. 9-17.

32. Plant, P.K., J.L. Owen, and M.W. Elliott, One year period prevalence study of respiratory acidosis in acute exacerbations of COPD: implications for the provision of non-invasive ventilation and oxygen administration. Thorax, 2000. 55(7): p. 550-4.

33. Plant, P.K., J.L. Owen, and M.W. Elliott, Early use of non-invasive ventilation for acute exacerbations of chronic obstructive pulmonary disease on general respiratory wards: a multicentre randomised controlled trial. Lancet, 2000. 355(9219): p. 1931-5.

34. Crisafulli, E., et al., Management of severe acute exacerbations of COPD: an updated narrative review. Multidiscip Respir Med, 2018. 13: p. 36.

35. Vitacca, M., et al., Non-invasive mechanical ventilation in severe chronic obstructive lung disease and acute respiratory failure: short- and long-term prognosis. Intensive Care Med, 1996. 22(2): p. 94-100.

36. Ozyilmaz, E., A.O. Ugurlu, and S. Nava, Timing of noninvasive ventilation failure: causes, risk factors, and potential remedies. BMC Pulm Med, 2014. 14: p. 19.

37. Schonhofer, B., et al., Clinical practice guideline: non-invasive mechanical ventilation as treatment of acute respiratory failure. Dtsch Arztebl Int, 2008. 105(24): p. 424-33.

38. Pacilli, A.M., et al., Determinants of noninvasive ventilation outcomes during an episode of acute hypercapnic respiratory failure in chronic obstructive pulmonary disease: the effects of comorbidities and causes of respiratory failure. Biomed Res Int, 2014. 2014: p. 976783.

39. Trupin, L., et al., The occupational burden of chronic obstructive pulmonary disease. Eur Respir J, 2003. 22(3): p. 462-9.

40. Dangers, L., et al., Dyspnoea in patients receiving noninvasive ventilation for acute respiratory failure: prevalence, risk factors and prognostic impact: A prospective observational study. Eur Respir J, 2018. 52(2).

41. Charlson, M.E., et al., A new method of classifying prognostic comorbidity in longitudinal studies: development and validation. J Chronic Dis, 1987. 40(5): p. 373-83. 


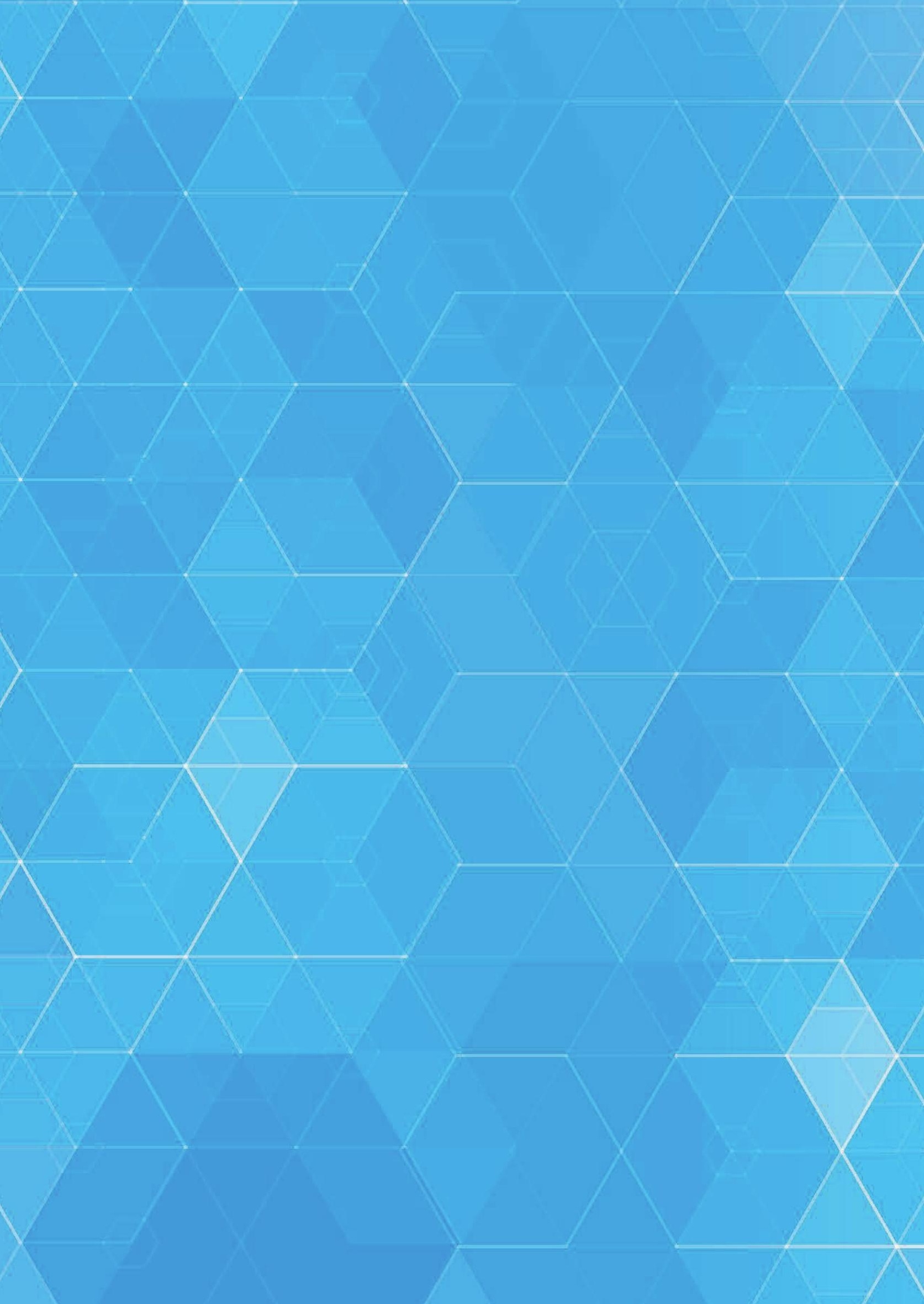




\section{Chapter 7}

\section{Nocturnal non-invasive ventilation in \\ COPD patients who remain hypercapnic \\ after ventilatory support for acute respiratory failure: a randomized, controlled, parallel-group study}

Acronym: RESCUE REspiratory Support in COPD after acUte Exacerbation

Fransien M. Struik, Roy T.M. Sprooten, Harm A.M. Kerstjens, Gerrie Bladder, Marianne Zijnen, Jerryll Asin, Nicolle A.M. Cobben, J.M. Vonk, Peter J.

Wijkstra

Published in Thorax 2014;69:826-834 


\section{Abstract}

\section{Introduction}

The effectiveness of non-invasive positive pressure ventilation (NIV) in COPD patients who remain hypercapnic after ventilatory support for acute respiratory failure (ARF) remains unclear. We investigated if nocturnal NIV in these patients prolongs the time to readmission for respiratory causes or death (primary endpoint) in the following 12 months.

\section{Methods}

201 COPD patients admitted to hospital with ARF and persisting hypercapnia $>48$ hours after termination of ventilatory support were randomized to NIV or standard treatment. Secondary outcomes were daytime arterial blood gasses, transcutaneous $\mathrm{PCO}_{2}$ during the night, lung function, health-related quality-of-life (HRQL), mood state, daily activities and dyspnoea.

\section{Results}

1year after discharge $65 \%$ versus $64 \%$ of patients (NIV vs standard treatment) were readmitted to hospital for respiratory causes or had died; time to event was not different $(\mathrm{p}=0.85)$. Daytime $\mathrm{PaCO}_{2}$ was significantly improved in NIV versus standard treatment $\left(\mathrm{PaCO}_{2} 0.5 \mathrm{kPa}(95 \% \mathrm{Cl} 0.04 ; 0.90 \mathrm{p}=0.03)\right)$ as was transcutaneous $\mathrm{PCO}_{2}$ during the night. HRQL showed a clear trend ( $p=0.054$, Severe Respiratory Insufficiency questionnaire) in favour of NIV. Number of exacerbations, lung function, mood state, daily activity levels or dyspnoea were not significantly different.

\section{Conclusions}

We could not demonstrate an improvement in time to readmission or death by adding NIV for 1 year in patients with prolonged hypercapnia after an episode of NIV for ARF. There is no reason to believe the NIV was not effective since daytime $\mathrm{PaCO}_{2}$ and nighttime $\mathrm{PaCO}_{2}$ improved. The trend for improvement in HRQL favouring NIV we believe nevertheless should be explored further. 


\section{Introduction}

Non-invasive positive pressure ventilation (NIV) has become an established treatment in patients with chronic obstructive pulmonary disease (COPD) who are admitted to hospital with acute respiratory failure (ARF) due to an exacerbation. ${ }^{1-4}$ As readmission and mortality rates in these patients are high ${ }^{5,6}$, the application of long-term nocturnal NIV in stable hypercapnic COPD has been subject of several studies. However, in a meta-analysis no clear benefits were documented. ${ }^{7-11}$ Reasons for these negative results were: (1) insufficient ventilatory support; (2) lack of proper monitoring during the night; (3) baseline $\mathrm{PaCO}_{2}$ too low; and (4) low adherence to NIV. The two long-term randomised controlled trials (RCTs) comparing NIV in addition to long-term oxygen therapy (LTOT) showed conflicting results: one demonstrated small improvements in daytime arterial carbon dioxide pressure ( $\mathrm{PaCO} 2$ ), dyspnoea and health related quality of life (HRQL) ${ }^{12}$ in favour of NIV whereas the other found an improvement in survival but at the cost of worsening HRQL. ${ }^{13}$

Two other studies investigated the effects of long-term NIV in the most severe subgroup of COPD patients; that is, patients with acute-on-chronic respiratory failure. These indicated a benefit from long-term NIV in patients who previously required mechanical ventilation for treatment of ARF and who remained hypercapnic thereafter. ${ }^{6,14}$ Unfortunately, these were both small studies and given the huge impact of COPD on mortality and morbidity ${ }^{15}$ further studies are urgently needed.

We hypothesized that providing nocturnal NIV alongside standard medical treatment to patients who remain hypercapnic after ventilatory support during ARF, would prolong the time to readmission for respiratory causes or death. Therefore, we conducted a RCT (randomized controlled trial) comparing nocturnal NIV with standard treatment for 1 year.

\section{Methods}

The RESCUE trial (REspiratory Support in COPD after acUte Exacerbation) was a multi-centre, prospective, randomized, controlled study recruiting COPD patients who were admitted to hospital with ARF and treated with ventilatory support throughout the Netherlands between December 1, 2007 and July 1, 2012. Two hundred and one patients with severe COPD from in total 47 hospitals were enrolled by 3 Centers for Home Mechanical Ventilation (University Medical Center Groningen, Maastricht University 
Medical Center and the Erasmus Medical Center, Rotterdam) with a follow-up of 12 months. Inclusion criteria were: 1) COPD, GOLD stage 3 and 4; [15] 2) >48 h independence from ventilatory support (invasive or non-invasive) for acute respiratory failure; 3) prolonged hypercapnia $\left(\mathrm{PaCO}_{2}>6.0 \mathrm{kPa}\right)$ during daytime at rest without oxygen or ventilatory support. The study was approved by the local Medical Ethics Committee of the University Medical Centre Groningen, University of Groningen, patients gave informed consent to participate in the study and, the trial was registered at the Nederland's Trial Register (NTR1100). For a more detailed description of the methods used, see Supplementary file.

\section{Randomization and intervention}

Patients were randomized to nocturnal NIV or standard treatment by a computer with minimization for $\mathrm{FEV}_{1}(\leq 0.8 \mathrm{~L}$ or $>0.8 \mathrm{~L}), \mathrm{PaCO}_{2}(\leq 7.0 \mathrm{kPa}$ or $>7.0 \mathrm{kPa})$, body mass index (BMI) $\left(\leq 30 \mathrm{~kg} / \mathrm{m}^{2}\right.$ or $\left.>30 \mathrm{~kg} / \mathrm{m}^{2}\right)$, ventilatory support (invasive or non-invasive ventilation) and rehabilitation (yes/no). Standard treatment consisted of optimal medical treatment as usual, with LTOT in case of clinically stable patients with a $\mathrm{PaO}_{2}<8.0 \mathrm{kPa}$ as indicated according to the Dutch Guideline. ${ }^{16}$

Nocturnal NIV was started by a nurse practitioner from the nearest home mechanical ventilation centre who visited the hospital where the patient was admitted. NIV was initiated by bi-level positive airway pressure in the spontaneous/timed mode (Synchrony, Respironics, INC, Murrysville, PA, USA) starting with a low back-up frequency of $12 / \mathrm{min}$ with the aim to: with the aim to: 1 ) maximally support respiration and; 2) and achieve normocapnia. NIV was initiated with an Inspiratory Positive Airway Pressure (IPAP) of $14 \mathrm{~cm} \mathrm{H}_{2} \mathrm{O}$ and gradually increased during trials to a maximal tolerated level. Expiratory Positive Airway Pressure (EPAP) was started at $4 \mathrm{~cm} \mathrm{H}_{2} \mathrm{O}$ and increased if auto-PEEP was present or when patients used respiratory muscles to trigger the ventilator. Respiratory rate (RR) was set as close as possible to the RR of the patient, with adjustments if the patient triggered too much. Inspiration to expiration time was $1: 3$, with a short rise time and then titrated on comfort and effectiveness. All patients were initially given ventilation by means of a full-face mask and humidification to maximise comfort. Effectiveness with NIV during the night was observed by monitoring the transcutaneous $\mathrm{O}_{2}$ saturation and $\mathrm{PCO}_{2}\left(\mathrm{PtCO}_{2}\right)$ with the TOSCA device (TOSCA 500, Linde Medical Sensors AG, Basel, Switzerland). ${ }^{17}$ Adherence to NIV was derived from time counters in the NIV device. 


\section{Measurements}

At baseline, arterial and lung function measurements were performed just before randomization. Baseline HRQL was assessed after randomization while transcutaneous measurements were performed during the night after patients were adjusted to NIV but before discharge of the initial admission for ARF. Lung function and arterial blood gas measurements were repeated after 3, 6 and 12 months during visits to their regular pulmonologist. Arterial blood gases were taken while breathing room air; however when patients were unable to stop oxygen therapy even for short periods, measurements were taken with their usual oxygen flow rate. The nurse practitioner also visited all patients at home after 3,6 and 12 months to collect survival, admission and exacerbation rates, HRQL and transcutaneous measurements, and if necessary to adjust NIV.

The long recruitment period enabled us to extend survival follow-up beyond 12 months to maximally 68 months for the first patients that entered the study.

HRQL was assessed by the Clinical COPD Questionnaire $(C C Q)^{18}$, the Maugeri Respiratory Failure Questionnaire-28 (MRF-28) ${ }^{19}$, the Chronic Respiratory Questionnaire self-reported $(C R Q)^{20}$, and the Severe Respiratory Insufficiency (SRI) questionnaire ${ }^{21}$, We also measured mood state (Hospital Anxiety and Depression scale $\left(\mathrm{HADS}^{22}\right.$, daily activity level (Groningen Activity and Restriction Scale (GARS) ${ }^{23}$ and dyspnoea (Medical Research Council (MRC). ${ }^{24}$

\section{Sample size calculations}

Time to event (readmission for respiratory cause or death) as primary outcome was used to determine the sample size, based on the assumption of an event free survival of $20 \%$ in the standard treatment group ${ }^{5}$ after 12 months and $40 \%$ in the NIV group. With an $\alpha$ of 0.05 and a power of $80 \%$, we needed 174 patients to detect the $20 \%$ difference in event free survival between groups. Estimating a maximum dropout rate of $15 \%, 200$ patients were needed.

\section{Statistical analysis}

The primary outcome and survival analysis was based on intention-to-treat (ITT) using Kaplan-Meier estimates and the log-rank test. A complete case analysis was performed for the remaining secondary outcomes comparing data of the patients who completed the 12 months (completers) as the number of drop-outs was considerable due to 
progression of the disease and reluctance to adhere to study requirements. Arterial blood gas measurements could not always be performed under similar conditions for every patient. Therefore, data were analyzed in two ways; first regardless of oxygen use or the amount of oxygen and secondly only comparing baseline and 12 month data when either the measurements were both on room air or both on oxygen at the same flow rate (also see supplementary files).

As not all patients were compliant to NIV, either not using it every night or refusing treatment altogether, a per protocol analysis comparing patients in the NIV group who used ventilation $>5$ hours/night with standard treatment was conducted based on results of a recent meta-analysis showing positive effects on change in arterial blood gasses in this subgroup. ${ }^{25}$ All statistical calculations were performed using IBM SPSS Statistics version 20 (IBM, New York).

\section{Results}

\section{Patients}

In all, 201 patients met eligibility criteria, 101 were randomized to NIV and 100 to standard treatment (Figure 7.1). At baseline, patient characteristics and demographics did not differ significantly between treatment groups for ITT as well as the completers (Table 7.1) except for the total number of admission days in the previous year. Reasons for dropping-out of the study are listed in the supplementary files. Four patients classified as dropouts crossed from standard treatment over to the NIV treatment during the study. They remained in the standard treatment group for all ITT-analyses.

\section{NIV settings + treatment compliance}

Patients randomized for NIV were discharged with a mean \pm SD IPAP of $19.2 \pm 3.4$, and EPAP of $4.8 \pm 1.0 \mathrm{~cm} \mathrm{H}_{2} \mathrm{O}$ respectively, mean RR on NIV of $15 \pm 3$ breaths/minute, an inspiration time of $1.1 \pm 0.3 \mathrm{~s}$ and a rise time of $1.8 \pm 1.1 \mathrm{~s}$. Settings were not different between the completers and the dropouts. $68 \%$ received oxygen combined with NIV during the night. Nearly all patients were ventilated through a full-face mask, one using a total face mask. Minor adjustments were made during the study to improve compliance, blood gasses and/or comfort. After 1 year the remaining 54 patients were ventilated with a mean IPAP of $21.0 \pm 3.4$ and an EPAP of $5.2 \pm 1.2 \mathrm{~cm} \mathrm{H} \mathrm{H}_{2} \mathrm{O}$. Two additional patients switched to a total face mask. 
Mean duration of NIV use per night until death or last follow-up was $6.3 \pm 2.4$ hours/night in the total group and $6.9 \pm 2.1$ in the completers. Patients included in the per protocol analysis (NIV $>5$ hours) reached an average of $7.7 \pm 1.5$ hours/night $(n=43)$.

Figure 7.1 Consort flow diagram.

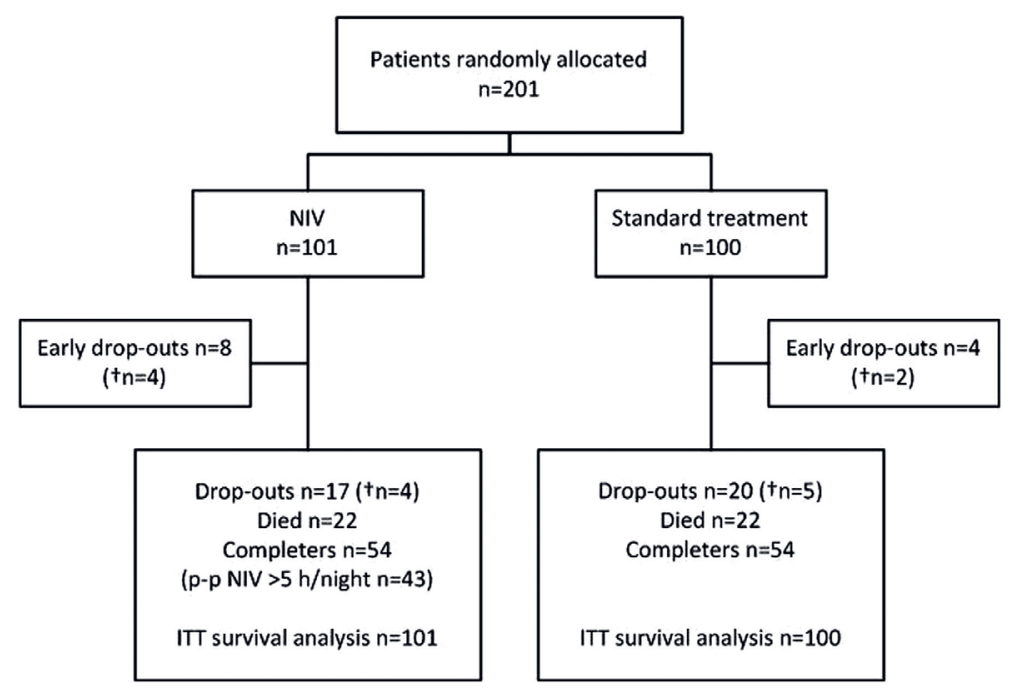

Early drop-outs: When patients refused or were not able to continue the protocol (either NIV or standard treatment) within 10 days after inclusion. Completers: number of patients included in the complete case analysis. Four patients classified as drop-outs crossed from standard therapy over to the NIV treatment during the study. ITT: number of patients included in the intention-to-treat analysis; $n$, numbers for analysis; $\mathrm{n}+$, number of patients who died within the drop-outs; NIV, non-invasive ventilation; $\mathrm{p}-\mathrm{p}$, included in the per protocol analysis of patients who used ventilation $>5$ hours/night.

\section{Time to event}

One year after discharge $65 \%$ vs. $64 \%$ of patients (NIV vs. standard treatment) were either readmitted to hospital for COPD or had died. Figure 7.2 shows the ITT event-free survival of both groups in the first year, which was not significantly different (mean number of days to event was 192 and 198 for the NIV and standard treatment group, respectively, $\mathrm{p}=0.85$ ). 
Table 7.1 Baseline characteristics.

\begin{tabular}{|c|c|c|c|c|}
\hline & $\begin{array}{c}\text { ITT } \\
\text { NIPPV } \\
n=101\end{array}$ & $\begin{array}{c}\text { ITT } \\
\text { Controls } \\
n=100\end{array}$ & $\begin{array}{c}\text { Completers } \\
\begin{array}{c}\text { NIV } \\
n=54\end{array}\end{array}$ & $\begin{array}{c}\text { Completers } \\
\text { Controls } \\
n=54\end{array}$ \\
\hline Age (years) & $63.92(8.6)$ & $63.5(7.9)$ & $61.9(7.9)$ & $62.3(7.9)$ \\
\hline Gender, n (\% female) & $60(59 \%)$ & $58(58 \%)$ & $36(67 \%)$ & $34(63 \%)$ \\
\hline Pack years & $38(0-140)$ & $44(0-125)$ & $38(0-125)$ & $38(0-125)$ \\
\hline $\mathrm{BMI}\left(\mathrm{kg} / \mathrm{m}^{2}\right)$ & $24.6(5.4)$ & $24.8(6.3)$ & $24.7(5.5)$ & $24.2(5.3)$ \\
\hline LTOT n (\%) & $76(75 \%)$ & $78(78 \%)$ & $38(70 \%)$ & 37 (69\%) \\
\hline Invasive/NIV (n) & $13(13 \%)$ & $12(12 \%)$ & $7(13 \%)$ & $10(19 \%)$ \\
\hline Duration ventilation $^{*}$ (days) & $5.0(1-19)$ & $5.0(1-24)$ & $5.5(2-19)$ & $5.0(1-23)$ \\
\hline $\operatorname{Admissions}^{\S}\left(\mathrm{yr}^{-1}\right)$ & $2.0(1-9)$ & $2.0(1-10)$ & $2.0(1-9)$ & $1.0(1-6)$ \\
\hline Total admission days/patient ${ }^{\S}\left(\mathrm{yr}^{-1}\right)$ & $28.5(8-108)$ & $22.0(6-115)^{*}$ & $29.0(8-100)$ & $20.0(7-115)^{*}$ \\
\hline Rehabilitation (\%) & $50(50 \%)$ & $51(51 \%)$ & $25(46 \%)$ & $30(56 \%)$ \\
\hline $\mathrm{FEV}_{1}(\mathrm{~L})$ & $0.67(0.23)$ & $0.65(0.23)$ & $0.70(0.24)$ & $0.70(0.25)$ \\
\hline $\mathrm{FEV}_{1}(\%$ pred $)$ & $25.6(7.8)$ & $25.7(8.6)$ & $26.9(7.7)$ & $27.6(8.6)$ \\
\hline FVC (\% pred) & $64.3(19.8)$ & $63.6(17.0)$ & $65.7(18.4)$ & $67.8(17.1)$ \\
\hline $\mathrm{FEV}_{1} / \mathrm{FVC}(\%)$ & $32.5(9.0)$ & $33.0(9.5)$ & $33.9(9.6)$ & $33.8(9.7)$ \\
\hline $\mathrm{PaCO}_{2}{ }^{9}(\mathrm{kPa})$ & $7.9(1.2)$ & $7.7(1.3)$ & $8.0(1.2)$ & $7.5(1.0)$ \\
\hline $\mathrm{PaO}_{2}{ }^{\curvearrowleft}(\mathrm{kPa})$ & $7.9(2.1)$ & $7.5(1.7)$ & $8.3(1.6)$ & $7.9(1.8)$ \\
\hline \multicolumn{5}{|l|}{ Medication, n (\%) } \\
\hline Inhaled corticosteroid & $86(85 \%)$ & $84(84 \%)$ & $43(81 \%)$ & $48(89 \%)$ \\
\hline Oral corticosteroids & $53(53 \%)$ & $38(38 \%)$ & $27(50 \%)$ & $17(32 \%)$ \\
\hline Beta agonists & $98(97 \%)$ & 97 (97\%) & $52(98 \%)$ & $53(98 \%)$ \\
\hline Anticholinergics & 94 (93\%) & 94 (94\%) & 49 (93\%) & $52(96 \%)$ \\
\hline Theophylline & $37(37 \%)$ & $27(27 \%)$ & $20(38 \%)$ & $16(30 \%)$ \\
\hline
\end{tabular}

Data presented as mean (SD) unless otherwise stated. Abbreviations: BMI, body mass index; Controls, standard treatment; F, female; FEV ${ }_{1}$, forced expiratory volume in one second; \% pred, \% predicted; FVC, forced vital capacity; ITT, intention-to-treat; LTOT, long-term oxygen therapy; $M$, male; $n$, numbers for analysis; NIV, non-invasive ventilation; $\mathrm{PaCO}_{2}$, arterial carbon dioxide tension; $\mathrm{PaO}_{2}$, arterial oxygen tension. \# type of ventilation during acute respiratory failure.

\section{Hospital admissions}

After 1 year, readmission to hospital for respiratory causes occurred in 56 vs. $57 \%$ in the NIV and the standard treatment group, respectively. The median (range) number of readmissions during the 12 months of follow-up did not differ significantly between groups, $(1.0(0-9)$ in NIV vs. $1.0(0-6)$ in the standard treatment group $p=0.23)$, nor did the median number of total days spent in hospital (7.0 (0-107) in NIV vs. 3.5 (0-77) for standard treatment) $(p=0.087))$.

\section{Survival}

The number of deaths after 1 year was 30 and 29 in the NIV and standard treatment group respectively with a mean number of survival days of 299 for NIV and 291 days for the standard treatment group ( $p=0.99)$ (see supplementary files for causes of death). 
Long-term survival is shown in Figure 7.3, with 63 deaths in the NIV group vs. 58 in the standard treatment group.

Figure 7.2 Kaplan-Meijer plot of time to event (readmission for respiratory cause or death) in COPD patients with persistent chronic respiratory failure after an episode of non-invasive ventilation (NIV) for acute respiratory failure.

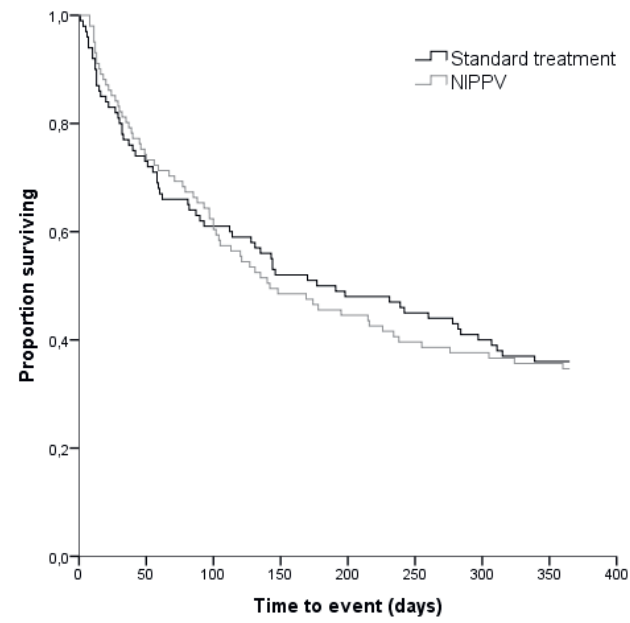

Figure 7.3 Long term survival curves for COPD patients with persistent chronic respiratory failure after an episode of non-invasive ventilation (NIV) for acute respiratory failure. Because of small numbers of patients followed up after 3 years the right-hand end of the survival plots remain uncertain.

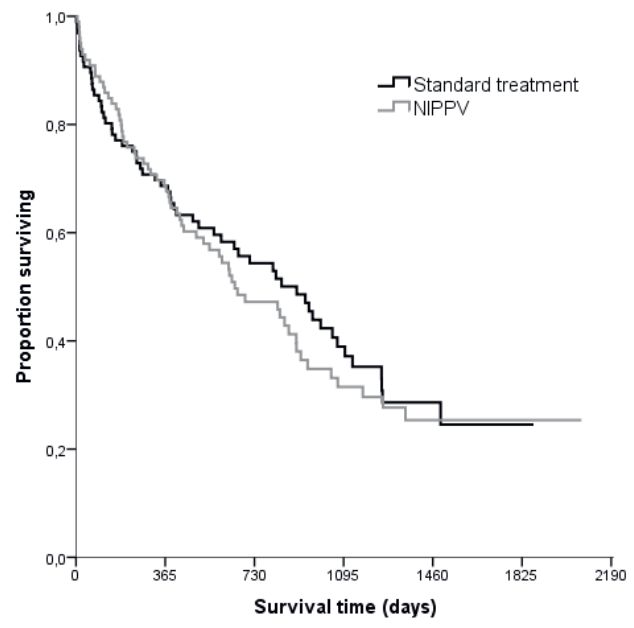




\section{Exacerbations}

No difference was observed between the annual median (range) number of exacerbations at home: $1.0(0-9)$ for the NIV and $2.0(0-14)$ for the standard treatment group $(p=0.26)$.

\section{Daytime arterial blood gases}

After 12 months, daytime $\mathrm{PaCO}_{2}$ improved significantly more in the NIV group compared to standard treatment (Table 7.2, Figure 7.4).

Figure 7.4 Daytime $\mathrm{PaCO}_{2}$ and $\mathrm{PaO}_{2}$ at baseline, 3, 6 and 12 months (means $\pm \mathrm{SD}$ ). Measurements performed in complete case group ( $n=54$ per group) regardless of oxygen use. NIV, non-invasive ventilation; $\mathrm{PaCO}_{2}$, arterial carbon dioxide tension (kPa); $\mathrm{PaO}_{2}$, arterial oxygen tension (kPa). *Change in $\mathrm{PaCO}_{2}$ was significantly better in the NIV group after 12 months $(\mathrm{p}<0.05)$.
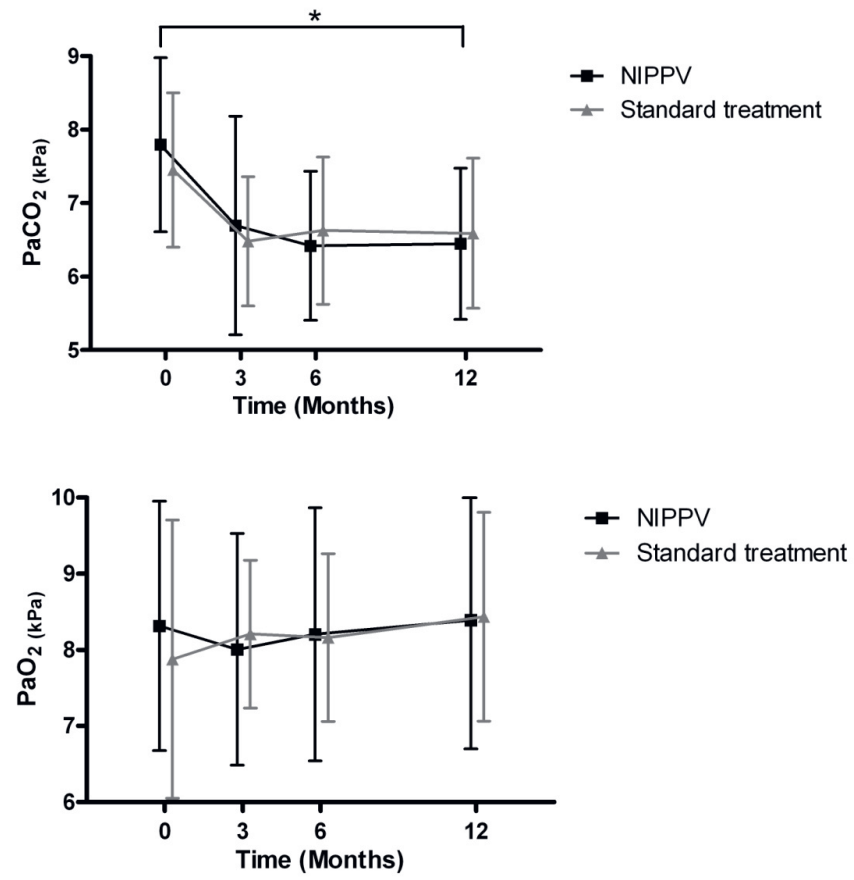


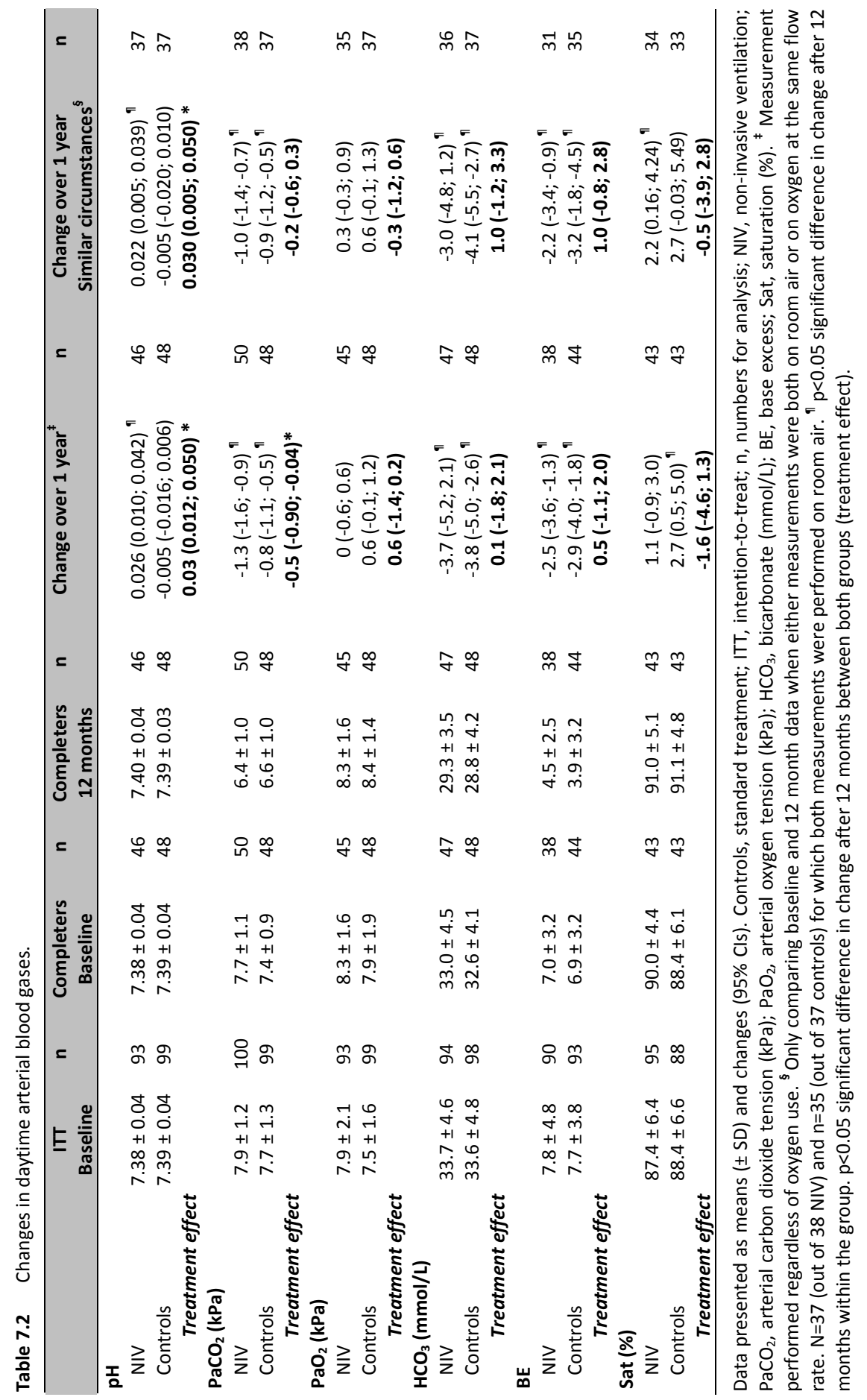




\section{Nocturnal gas exchange measurements}

The mean nocturnal $\mathrm{PtcCO}_{2}$ measured during the night before discharge out of hospital in the ITT group was significantly better in patients on NIV compared to standard treatment (mean difference $-0.8 \mathrm{kPa}(95 \% \mathrm{Cl}-1.3$ to $-0.4 ; \mathrm{p}<0.01)$ ). In the completers, this effect was not found at baseline but was present after 12 months; mean nocturnal $\mathrm{PtcCO}_{2}$ was significantly better in the NIV group compared to standard treatment (mean difference $-0.6 \mathrm{kPa}(95 \% \mathrm{Cl}-1.1$ to $-0.1 ; \mathrm{p}=0.03)$ ).

\section{Lung function}

Mean $\mathrm{FEV}_{1}$ and vital capacity did not change significantly after 12 months within the NIV group or standard treatment group nor was there a significant change between groups (Table 7.3).

Table 7.3 Changes in lung function.

\begin{tabular}{|c|c|c|c|c|c|c|c|c|}
\hline & $\begin{array}{c}\text { ITT } \\
\text { Baseline }\end{array}$ & $n$ & $\begin{array}{c}\text { Completers } \\
\text { Baseline }\end{array}$ & $\mathrm{n}$ & $\begin{array}{l}\text { Completers } \\
12 \text { months }\end{array}$ & $n$ & $\begin{array}{c}\text { Change over } \\
1 \text { year }\end{array}$ & $\mathrm{n}$ \\
\hline \multicolumn{9}{|l|}{ FEV $_{1}$ (litres) } \\
\hline NIV & $0.67(0.23)$ & 99 & $0.70(0.24)$ & 48 & $0.71(0.30)$ & 48 & $0.01(-0.05 ; 0.08)$ & 48 \\
\hline Controls & $0.65(0.23)$ & 95 & $0.72(0.26)$ & 46 & $0.76(0.32)$ & 46 & $0.04(-0.03 ; 0.11)$ & 46 \\
\hline Treatment effect & & & & & & & $-0.024(-0.12 ; 0.07)$ & \\
\hline \multicolumn{9}{|l|}{ VC (litres) } \\
\hline NIV & $2.1(0.7)$ & 98 & $2.1(0.70)$ & 49 & $2.2(0.78)$ & 49 & $0.09(-0.09 ; 0.27)$ & 49 \\
\hline Controls & $2.3(0.8)$ & 95 & $2.3(0.75)$ & 46 & $2.4(0.74)$ & 46 & $0.13(-0.08 ; 0.34)$ & 46 \\
\hline Treatment effect & & & & & & & $-0.036(-0.31 ; 0.23)$ & \\
\hline
\end{tabular}

Data presented as means $( \pm \mathrm{SD})$ and changes $(95 \% \mathrm{Cls})$. Controls, standard treatment. There were no significant changes after 12 months in lung function within or between the groups.

$\mathrm{FEV}_{1}$, forced expiratory volume in one second, post-bronchodilator; ITT, intention-to-treat; n, numbers for analysis; NIV, non-invasive ventilation; VC, vital capacity.

\section{Health related quality of life}

HRQL, activities of daily living, anxiety/depression scores and dyspnoea improved in both groups but there was no significant difference between the groups (Table 7.4 and see supplementary files). HRQL measured by the SRI total score showed a clear trend $(p=0.054)$ with additionally significant differences between groups on the sub domains 'attendant symptoms and sleep' and 'social relationships' in favour of the NIV group (see supplementary files). 
Table 7.4 Changes in Health-Related Quality-of-life measurements.

\begin{tabular}{|c|c|c|c|c|c|c|c|c|}
\hline & $\begin{array}{c}\text { ITT } \\
\text { Baseline }\end{array}$ & $\mathbf{n}$ & $\begin{array}{c}\text { Completers } \\
\text { Baseline }\end{array}$ & $\mathbf{n}$ & $\begin{array}{l}\text { Completers } \\
12 \text { months }\end{array}$ & $\mathbf{n}$ & $\begin{array}{c}\text { Change over } \\
1 \text { year }\end{array}$ & $\mathbf{n}$ \\
\hline \multicolumn{9}{|l|}{ CCQ-Total } \\
\hline NIV & $3.37 \pm 1.2$ & 96 & $3.4 \pm 1.2$ & 48 & $2.9 \pm 1.1$ & 48 & $-0.5(-0.2 ;-0.8)^{9}$ & 48 \\
\hline Controls & $3.31 \pm 1.06$ & 90 & $3.2 \pm 1.0$ & 51 & $2.8 \pm 1.1$ & 51 & $-0.5(-0.8 ;-0.1)^{9}$ & 51 \\
\hline Treatment effect & & & & & & & $-0.04(-0.46 ; 0.38)$ & \\
\hline \multicolumn{9}{|l|}{ MRF-28-Total } \\
\hline NIV & $60.88 \pm 23.60$ & 99 & $58.3 \pm 24.3$ & 50 & $51.0 \pm 24.8$ & 50 & $-7.3(-12.5 ; 2.1)^{9}$ & 50 \\
\hline Controls & $60.28 \pm 23.89$ & 90 & $55.3 \pm 24.2$ & 51 & $49.4 \pm 24.2$ & 51 & $-5.8(-10.8 ;-0.8)^{n}$ & 51 \\
\hline Treatment effect & & & & & & & $-1.5(-8.59 ; 5.7)$ & \\
\hline \multicolumn{9}{|l|}{ CRQ-Total } \\
\hline NIV & $3.47 \pm 1.10$ & 100 & $3.5 \pm 1.1$ & 50 & $4.2 \pm 1.2$ & 50 & $0.7(0.4 ; 1.1)^{9}$ & 50 \\
\hline Controls & $3.59 \pm 1.08$ & 89 & $3.6 \pm 1.1$ & 50 & $4.4 \pm 1.1$ & 50 & $0.7(0.5 ; 1.0)^{n}$ & 50 \\
\hline Treatment effect & & & & & & & $0.01(-0.4 ; 0.4)$ & \\
\hline \multicolumn{9}{|l|}{ SRI-Total } \\
\hline NIV & $48.14 \pm 14.97$ & 100 & $47.9 \pm 15.1$ & 50 & $55.0 \pm 15.4$ & 50 & $7.0(3.4 ; 10.7)^{9}$ & 50 \\
\hline Controls & $51.33 \pm 15.87$ & 90 & $53.6 \pm 16.9$ & 51 & $55.8 \pm 16)$. & 51 & $2.2(-1.2 ; 5.6)$ & 51 \\
\hline Treatment effect & & & & & & & $4.8(-0.1 ; 9.7)$ & \\
\hline \multicolumn{9}{|l|}{ GARS-Total } \\
\hline NIV & $36.66 \pm 7.50$ & 100 & $36.3 \pm 9.4$ & 50 & $34.6 \pm 9.4$ & 50 & $-1.6(-3.3 ; 0.1)$ & 50 \\
\hline Controls & $36.76 \pm 8.54$ & 90 & $34.7 \pm 8.8$ & 51 & $32.7 \pm 8.2$ & 51 & $-2.0(-4.1 ; 0.1)$ & 51 \\
\hline Treatment effect & & & & & & & $0.4(-2.3 ; 3.0)$ & \\
\hline \multicolumn{9}{|l|}{ HADS-Total } \\
\hline NIV & $15.88 \pm 9.24$ & 98 & $15.6 \pm 8.8$ & 48 & $13.9 \pm 8.8$ & 48 & $-1.7(-4.0 ; 0.6)$ & 48 \\
\hline Controls & $14.48 \pm 8.83$ & 87 & $13.1 \pm 9.1$ & 50 & $12.7 \pm 9.3$ & 50 & $-0.4(-2.2 ; 1.3)$ & 50 \\
\hline Treatment effect & & & & & & & $-1.3(-4.1 ; 1.6)$ & \\
\hline \multicolumn{9}{|l|}{ MRC } \\
\hline NIV & $3.9 \pm 1.0$ & 98 & $3.8 \pm 1.0$ & 49 & $3.4 \pm 1.4$ & 49 & $-0.4(-0.7 ;-0.01)^{n}$ & 49 \\
\hline Controls & $3.8 \pm 1.0$ & 90 & $3.6 \pm 1.1$ & 51 & $3.3 \pm 1.2$ & 51 & $-0.3(-0.7 ; 0.1)$ & 51 \\
\hline Treatment effect & & & & & & & $-0.05(-0.6 ; 0.5)$ & \\
\hline
\end{tabular}

Data presented as means ( \pm SD) and changes ( $95 \% \mathrm{Cls}) . \mathrm{CCQ}$, Clinical COPD Questionnaire; Controls, standard treatment; CRQ, Chronic Respiratory Questionnaire; GARS; Groningen Activity and Restriction Scale HADS, Hospital Anxiety and Depression Scale (separate scores for anxiety and depression domain); MRC, Medical Research Council dyspnoea scale; MRF-28, Maugeri Respiratory Failure-28 questionnaire; $n$, numbers for analysis; NIV, non-invasive ventilation; SRI, Severe Respiratory Insufficiency questionnaire. " $p<0.05$ significant difference in change after 12 months within the group.

\section{Per protocol analysis}

All outcomes were similar for the per protocol analysis comparing patients with NIV $>5$ hours/night with standard treatment. 


\section{Discussion}

Thus far, this is the largest randomized, controlled multi center study reporting on the effects of NIV in COPD patients with prolonged hypercapnia after an episode of NIV for ARF. It showed that nocturnal NIV does not prolong the time to readmission for respiratory causes or death in COPD patients who remain hypercapnic after ventilatory support during ARF. Compared to standard treatment alone, NIV improves daytime $\mathrm{PaCO}_{2}$ and nocturnal transcutaneous $\mathrm{PCO}_{2}$ but does not improve survival, number of readmissions, exacerbations, lung function, HRQL, mood state, daily activity levels or dyspnoea.

In this large study, the design was practical and executed as in 'real-life', which make outcomes more broadly applicable (patients remained in their own hospital and blood gas and lung function measurements were performed during their regular visits to their own pulmonologist), but does make it difficult to compare our outcomes with previous studies in this field and therefore necessitates further discussion and comparison.

First, Funck et al. ${ }^{14}$ reported positive results in an RCT including similar patients (COPD patients remaining hypercapnic after ARF requiring mechanical ventilation), showing a higher probability of clinical worsening in the control (withdrawal) group compared with the NIV group. A crucial difference in trial designs is that all patients first received NIV for 6 months after which one group was randomized to stop NIV (withdrawal group). Clinical worsening was defined as 1) an intensive care unit admission for treatment of acute-on-chronic respiratory failure requiring mechanical ventilation, but for the withdrawal group escalation additionally comprised of 2) voluntary resumption of NIV due to severe dyspnoea and 3) re-institution of NIV due to progressive hypercapnia. A pitfall of this approach was, as the authors also note themselves, that by definition the achievable endpoints depended on the group allocation of the patient as the NIV group could only qualify for the first endpoint. Results after 12 months followup, showed that ten patients in the withdrawal group versus two in the NIV group experienced clinical worsening $(p=0.0018)$. But upon looking more closely and, comparing only the endpoint which was achievable for both groups, only three out of the initial ten patients in the withdrawal group (vs. two) were admitted to the ICU for acute-on-chronic respiratory failure. Other positive outcomes in favor of NIV were a significant difference in 6-min walking distance after 3 months and a slightly lower resting daytime $\mathrm{pH}$ in the withdrawal group after 12 months. Our data show a higher daytime $\mathrm{pH}$ in the NIV group, but we did not consider this improvement clinically relevant (as patients were not acidic at baseline). We could not perform the 6 -minute 
walking test since most patients were too weak to perform this test adequately and safely, raising ethical concerns.

Second, in a randomized pilot trial by Cheung and colleagues ${ }^{6}$ with similar inclusion criteria as in our study, chronic home NIV was compared to placebo NIV (CPAP of $5 \mathrm{~cm}$ $\mathrm{H}_{2} \mathrm{O}$ ). In this study primary outcome was recurrent severe COPD exacerbation with acute hypercapnic respiratory failure (AHRF), resulting in NIV, intubation or death within the following year. The proportion of patients developing this condition in 1 year was $38.5 \%$ in the NIV vs. $60.2 \%$ in the placebo group ( $p=0.039)$. Compared to our study these patients were older (mean age 70) and had a lower mean BMI (19.2) suggesting a group of patients in worse condition than in the present study. The authors point out that ethical concerns led them to be prudent and to act upon severe enough exacerbations by instituting NIV, which could indicate a bias towards earlier conversion to NIV in the CPAP group and a possible explanation for the difference in proportions between groups. Furthermore, this trial did not find a difference in survival, arterial blood gasses and adverse events between groups.

Third, our power analysis was based on the study by Chu et al. ${ }^{5}$, who found that $80 \%$ of COPD patients with AHRF and who were successfully treated by NIV were readmitted to hospital for respiratory problems within the following year. Our primary outcome consisted of readmission for respiratory causes but also death, probably leading to higher percentages of patients in which 'the event' would occur. Ideally, it would be more appropriate to power on pilot data from our own country or a similar European Healthcare system but as this was the only study at that time we had to work with these numbers. We hypothesized that in our study the NIV group would have a $20 \%$ lower prevalence of readmission for respiratory causes than the standard treatment group (i.e. $60 \%$ prevalence of re-admission). However, our study shows a much lower prevalence of re-admission in both the NIV and but also the standard treatment group (56 and $57 \%$, respectively). A possible explanation could be that patients recruited in the study by Chu were older (mean age 73.2) and had a lower BMI (20.2), indicating a worse physical condition. Our results do show similarities with a Spanish study ${ }^{26}$ which was published during our inclusion phase, reporting readmission rates of $66 \%$ due to respiratory complications in COPD patients who survived an acute exacerbation with ARF that required NIV.

As patients were included during an admission for ARF, we anticipated blood gasses to improve in the first months after discharge from hospital in both groups. Although $\mathrm{PaCO}_{2}$ was better in the NIV group after 12 months, we did not expect to see the same 
level of improvement in $\mathrm{PaCO}_{2}$ in the standard treatment group in the first 3 months (figure 4). Ideally, it would be more appropriate to power on pilot data from our own country or a similar European Healthcare system but as this was the only study at that time we had to work with these numbers.

Another possible subgroup analyses for the future concerns the need for supplementary oxygen. This subgroup is likely to indicate a more severe disease state and this would be a group expected to have greater therapeutic benefit with chronic NIV.

In a subgroup analysis from a recent meta-analysis on the effects of NIV in stable COPD ${ }^{25}$, a significant change in $\mathrm{PaCO}_{2}$ after 3 months within the NIV group was found for patients with a baseline $\mathrm{PaCO}_{2}$ of at least $7.3 \mathrm{kPa}(55 \mathrm{~mm} \mathrm{Hg})$, for patients who used NIV for at least $5 \mathrm{~h}$ per night as well as for patients ventilated with IPAP levels of at least $18 \mathrm{~cm} \mathrm{H}_{2} \mathrm{O}$, when compared to patients with lower IPAP levels, lower adherence or lower levels of hypercapnia. In the current study, baseline $\mathrm{PaCO}_{2}$ and change in $\mathrm{PaCO}_{2}$ after 12 months of ventilation were significantly correlated $(r=-0.6, p<0.001)$. Levels of IPAP and the number of hours of NIV/night were not correlated with change in $\mathrm{PaCO}_{2}$ (data not shown). Our data show that $26 \%$ of all patients in the standard treatment group had become eucapnic after 3 months' time. If we extrapolate this number to the NIV group, this proportion of patients is less likely to benefit from chronic NIV. This failure to demonstrate a dose-response in number of hours and change in $\mathrm{PaCO} 2$ is therefore likely due to the 'dilution' of the prolonged hypercapnic patient cohort by patients who recover and improve and become eucapnic over a longer timeframe ( $>48 \mathrm{~h}$ in the current trial) after an acute decompensated exacerbation, that is, patients who did not have acute-on-chronic respiratory failure but AHRF. Possibly, in future studies, selection of patients should be based on the level of hypercapnia after 3 months of recovering at home, before initiating home NIV. However, data from within the EU have demonstrated the high mortality of this patient group in the months following an AE requiring ventilatory support and although this trial may have evaluated patients too earlier the optimum timing remains to be proven. $^{27}$

A limitation in our study was our inability to obtain blood gasses on patient's standard oxygen level, as patients were reluctant to undergo testing twice. Although our aim was to only obtain samples on room air, a portion of patients refused to stop their oxygen, making comparison of data difficult. Nevertheless, patients on NIV were adequately 
ventilated during the night as their mean nocturnal PtcCO2 was significantly better compared with standard treatment.

In an RCT on NIV in stable COPD, Clini et al. ${ }^{12}$ did not find a difference in $\mathrm{PaCO}_{2}$ between NIV and the control group (LTOT) during room air breathing, whereas they did show a significant difference when measured on patients' usual oxygen regimen.

Also, long-term oral corticosteroid treatment is suggested to negatively impact on readmission rates and outcome. Since NIV patients more frequently received oral corticosteroids (ITT $53 \%$ vs $38 \%$ ) this could also have affected the results.

The fact that we were able to obtain extensive data on HRQL is unique. During our study, we published an article ${ }^{28}$ in which reliability and validity of the CCQ, CRQ, MRF-28 and SRI questionnaires was assessed and compared from our patients at baseline. Although, all four questionnaires were feasible in these type of patients, the SRI scored tested most appropriate. This is reflected in our data after 12 months which show a clear trend towards improved HRQL as measured by the SRI total score. Two out of the seven sub domains were significantly improved in the NIV group compared to standard treatment. The very opposite was reported in the RCT by McEvoy et al. ${ }^{13}$ who demonstrated a survival advantage of chronic NIV in hypercapnic stable COPD but accompanied by a deterioration in HRQL (measured by the more general St. Georges Respiratory Questionnaire). We believe this emphasizes the need to consistently use severity-specific HRQL questionnaires like the SRI and MRF-28 to further explore the possible benefits of NIV in future research, for instance also the supportive role of NIV in advanced care planning.

\section{Conclusions}

We could not demonstrate an improvement in time to readmission or death by adding NIV for one year in COPD patients who remained hypercapnic after an episode of NIV for ARF. There is no reason to believe the NIV was not effective since daytime $\mathrm{PaCO}_{2}$ and night-time $\mathrm{PtCO}_{2}$ improved. The trend for improvement in HRQL favouring NIV we believe nevertheless should be explored further. 


\section{Supplementary material}

\section{METHODS}

\section{Patients}

Exclusion criteria were: 1) age $<18$ or $\geq 80$ years; 2) significant bronchiectasis with recurrent infections; 3) significant heart failure; 4) kyphoscoliosis; 5) neuromuscular disease; 6) obstructive sleep apnoea (Apnoea Hypopnea Index: AHI >15 /h); 7) current use of Continuous Positive Airway Pressure (CPAP) or Bi-level Positive Airway Pressure (BiPAP); 8) insufficient motivation for chronic ventilatory support; 9) social circumstances making chronic ventilatory support at home impossible; 10) other diagnoses limiting life expectations.

\section{Home Mechanical Ventilation Center}

In The Netherlands we have 4 legitimated and highly experienced Home Mechanical Ventilation Centers. Patients requiring chronic ventilation at home are sent in from all hospitals in the country to be established on mechanical ventilation by these centers. The nurse practitioners are allowed to establish long-term NIV also in external hospitals and deliver care at home. For this trial as a form of quality assurance, we set up a standardized protocol for establishment and NIV set up and organized a startup meeting to make sure everybody worked according to the same standard and regulations. Also, all centers worked with the same machines.

\section{Intervention}

Patients were instructed to use NIV during the night whilst asleep and, were advised to use NIV during the day/nap times if desired.

\section{Measurements}

An overnight polygraphy or Polysomnography was performed in patients with a body mass index $\geq 30 \mathrm{~km} / \mathrm{m}^{2}$, or in patients with complaints of excessive snoring, disrupted sleep or morning headache to exclude Obstructive Sleep Apnea Syndrome.

Survival, admission rates and days in hospital were checked in hospital registers and with patients general practitioner. Exacerbations at home were registered in a diary by the patient themselves. 
An exacerbation was defined using a modified version of the definition of RodriguezRoisin, as an event in the natural course of the disease characterized by a change in the patient's baseline dyspnoea, cough, and/or sputum that is beyond day-to-day variations, is acute in onset, and treated with antibiotics and/or prednisolone in patients with COPD. ${ }^{29}$

\section{Lung function}

Lung function measurements included routine spirometry by means of a pneumotachograph and according to ERS criteria. ${ }^{30}$ Absolute values and \% pred. according to normal values.

\section{Transcutaneous measurement}

Transcutaneous monitoring during the night was performed using the $\operatorname{TOSCA}^{17}$ to measure skin-surface $\mathrm{PO}_{2}$ and $\mathrm{PCO}_{2}$ to provide estimates of arterial partial pressure of oxygen and carbon dioxide $\left(\mathrm{PaO}_{2}\right.$ and $\left.\mathrm{PaCO}_{2}\right)$ and saturation. The device induces hyperperfusion by local heating of the skin of the earlobe and measures the partial pressure of oxygen and carbon dioxide electrochemically. The mean nocturnal $\mathrm{PtcCO}_{2}$ was measured during the night before discharge out of hospital to see if patients were adequately adjusted to NIV. Patients in the standard treatment did not receive NIV during this measurement.

\section{Health related quality of life questionnaires}

Patients completed the following questionnaires concerning health related quality of life; the Clinical COPD Questionnaire (CCQ) [18], the Chronic Respiratory Questionnaire self-reported $(\mathrm{CRQ})^{31}$, the Maugeri Respiratory Failure Questionnaire -28 (MRF-28) ${ }^{19}$ and the Severe Respiratory Insufficiency (SRI) questionnaire. ${ }^{21}$ The CCQ is a selfadministered, 10-item questionnaire which can be divided into three domains: symptom, functional state and mental state. Scores range from 0 to 6 with high scores indicating extremely poor health status. The CRQ (self-reported) contains 20 items and measures physical function and emotional function, divided into four domains: dyspnoea, fatigue, emotion and mastery. Scores range from 1 (worse) to 7 (best). The MRF-28 contains 28 items which are divided into 3 domains; daily activity, cognitive function and invalidity. The scores range from 0 (best) to 100 (worse). The SRI contains seven domains covering 49 items: respiratory complaints, physical functioning, attendant symptoms and sleep, social relationships, anxiety, psychological well-being 
and social functioning. Scoring ranges between $0-100$, with high scores representing better HRQL.

\section{Other measurements}

The Groningen Activity and Restriction Scale (GARS) assesses activity and disability of daily living and consists of 18 items. ${ }^{23}$ The Hospital Anxiety and Depression Scale (HADS) was used to determine levels of depression and anxiety. ${ }^{22}$ It consists of 14 questions from which seven are on detection of anxiety and seven on depression (two subscales). Dyspnoea was measured using the Medical Research Council scale (MRC). ${ }^{24}$

We could not perform the 6-minute walking test since most patients were too weak to perform this test adequately and safely, raising ethical concerns. Our alternative of measuring walking distance with a pedometer also fell short, as we noticed that in the patients who barely walk, shuffling occurs leading to an underestimation as the pedometer does not always detect their effort and thus real number of steps per day.

\section{Statistical analysis}

Continuous variables were summarized as means and standard deviations, or medians and range depending on their distribution. Changes within groups were compared using the paired sample T-test. Between group comparisons of continuous variables were performed using the independent samples T-test for variables with a normal distribution or the Mann-Whitney $U$ test for variables with a non-normal distribution. Difference in change within groups and treatment effect between groups are shown as means with associated $95 \%$ confidence intervals (C.I.) and p-value ( $p<0.05$ was considered statistically significant).

Table S7.1 Reasons for dropping out of study.

\begin{tabular}{lcc}
\hline & NIV & Standard treatment \\
& $\mathbf{n = 2 5}$ & $\mathbf{n = 2 4}$ \\
\hline Lack of motivation & 15 & 6 \\
Unable to come for testing\# & 0 & - \\
Discomfort associated with treatment & 8 & \\
Critical intercurrent illness: & & 0 \\
Dementia & 1 & 0 \\
CVA & 1 & 4 \\
Switch to NIV & - & \\
\hline
\end{tabular}

CVA cerebrovascular accident; $n$, numbers for analysis NIV, non-invasive ventilation. 
Table S7.2 Causes of death.

\begin{tabular}{lcccc}
\hline & $\begin{array}{c}\text { ITT } \\
\text { NIV } \\
\mathbf{n = 3 0}\end{array}$ & $\begin{array}{c}\text { ITT } \\
\text { Standard treatment } \\
\mathbf{n = 2 9}\end{array}$ & $\begin{array}{c}\text { Non-drop outs } \\
\text { NIV } \\
\mathbf{n = 2 2}\end{array}$ & $\begin{array}{c}\text { Non-drop outs } \\
\text { Standard treatment } \\
\mathbf{n = 2 2}\end{array}$ \\
\hline Respiratory causes & 21 & 25 & 15 & 20 \\
Natural cause & 3 & 3 & 3 & 1 \\
Pneumothorax & 1 & 1 & 1 & 1 \\
Cardiac disease & 3 & & 1 & \\
Lung carcinoma & 1 & & 1 & \\
Missing & 1 & & 1 & \\
\hline
\end{tabular}

ITT, intention-to-treat; $n$, numbers for analysis NIV, non-invasive ventilation.

Table S73 Changes in the Clinical COPD Questionnaire (CCQ) sub domains.

\begin{tabular}{|c|c|c|c|c|c|c|c|c|}
\hline & $\begin{array}{c}\text { ITT } \\
\text { Baseline } \\
\end{array}$ & $n$ & $\begin{array}{c}\text { Completers } \\
\text { Baseline } \\
\end{array}$ & $\mathrm{n}$ & $\begin{array}{c}\text { Completers } \\
12 \text { months } \\
\end{array}$ & & $\begin{array}{c}\text { Change over } \\
1 \text { year }\end{array}$ & $n$ \\
\hline \multicolumn{9}{|l|}{ CCQ-Total } \\
\hline NIV & $3.4 \pm 1.2$ & 96 & $3.4 \pm 1.2$ & 48 & $2.9 \pm 1.1$ & 48 & $-0.5(-0.2 ;-0.8)^{9}$ & 48 \\
\hline Controls & $3.3 \pm 1.2$ & 90 & $3.2 \pm 1.0$ & 51 & $2.8 \pm 1.1$ & 51 & $-0.5(-0.8 ;-0.1)^{9}$ & 51 \\
\hline Treatment effect & & & & & & & $-0.04(-0.46 ; 0.38)$ & \\
\hline \multicolumn{9}{|l|}{ Symptom } \\
\hline NIV & $3.1 \pm 1.2$ & 99 & $3.0 \pm 1.2$ & 51 & $2.7 \pm 1.2$ & 51 & $-0.3(-0.6 ; 0.1)$ & 51 \\
\hline Controls & $3.1 \pm 1.1$ & 90 & $3.2 \pm 1.1$ & 51 & $2.8 \pm 1.1$ & 51 & $-0.4(-0.7 ;-0.04)^{9}$ & 51 \\
\hline Treatment effect & & & & & & & $-0.09(-0.39 ; 0.56)$ & \\
\hline \multicolumn{9}{|l|}{ Functional } \\
\hline NIV & $4.1 \pm 1.4$ & 99 & $4.1 \pm 1.5$ & 49 & $3.5 \pm 1.5$ & 49 & $-0.5(-0.9 ;-0.2)^{9}$ & 49 \\
\hline Controls & $4.0 \pm 1.4$ & 90 & $3.9 \pm 1.4$ & 51 & $3.4 \pm 1.4$ & 51 & $-0.5(-0.9 ;-0.1)^{\Upsilon}$ & 51 \\
\hline Treatment effect & & & & & & & $-0.07(-0.6 ; 0.5)$ & \\
\hline \multicolumn{9}{|l|}{ Mental } \\
\hline NIV & $2.6 \pm 1.8$ & 96 & $2.7 \pm 1.8$ & 50 & $1.7 \pm 1.3$ & 50 & $-1.0(-1.4 ;-0.6)^{9}$ & 50 \\
\hline Controls & $2.3 \pm 1.7$ & 90 & $2.2 \pm 1.6$ & 51 & $1.6 \pm 1.5$ & 51 & $-0.6(-1.0 ;-0.2)^{9}$ & 51 \\
\hline Treatment effect & & & & & & & $-0.40(-1.0 ; 0.2)$ & \\
\hline
\end{tabular}

Data presented as means ( \pm standard deviation) and changes ( $95 \%$ confidence intervals). CCQ, Clinical COPD Questionnaire; ITT, intention-to-treat; $n$, numbers for analysis; NIV, non-invasive ventilation. " $p<0.05$ significant difference in change after 12 months within the group. ${ }^{*} p<0.05$ significant difference in change after 12 months between both groups (treatment effect). Low CCQ scores indicate high quality of life. A negative treatment effect signifies a bigger improvement after 12 months for the NIV group compared to standard treatment. 
Table S7.4 Changes in the Maugeri Respiratory Questionnaire-28 (MRF-28) sub domains.

\begin{tabular}{|c|c|c|c|c|c|c|c|c|}
\hline & $\begin{array}{c}\text { ITT } \\
\text { Baseline } \\
\end{array}$ & $n$ & $\begin{array}{c}\text { Completers } \\
\text { Baseline } \\
\end{array}$ & $n$ & $\begin{array}{l}\text { Completers } \\
12 \text { months } \\
\end{array}$ & $n$ & $\begin{array}{c}\text { Change over } \\
1 \text { year }\end{array}$ & $\mathbf{n}$ \\
\hline \multicolumn{9}{|l|}{ MRF-28-Total } \\
\hline NIV & $60.9 \pm 23.6$ & 99 & $58.3 \pm 24.3$ & 50 & $51.0 \pm 24.8$ & 50 & $-7.3(-12.5 ;-2.1)^{\pi}$ & 50 \\
\hline Controls & $60.3 \pm 23.9$ & 90 & $55.3 \pm 24.2$ & 51 & $49.4 \pm 25.0$ & 51 & $-5.8(-10.8 ;-0.8)^{9}$ & 51 \\
\hline Treatment effect & & & & & & & $-1.5(-8.6 ; 5.7)$ & \\
\hline \multicolumn{9}{|l|}{ Daily activities } \\
\hline NIV & $62.3 \pm 28.4$ & 99 & $62.0 \pm 29.9$ & 50 & $54.5 \pm 35.4$ & 50 & $-7.5(-15.09 ; 0.03)$ & 50 \\
\hline Controls & $61.7 \pm 30.5$ & 90 & $55.6 \pm 30.7$ & 51 & $46.3 \pm 29.2$ & 51 & $-9.3(-16.1 ;-2.4)^{9}$ & 51 \\
\hline Treatment effect & & & & & & & $1.7(-8.4 ; 11.8)$ & \\
\hline \multicolumn{9}{|l|}{ Cognition } \\
\hline NIV & $48.5 \pm 41.0$ & 99 & $44.5 \pm 40.8$ & 50 & $33.7 \pm 36.3$ & 50 & $-10.8(-22.5 ; 0.8)$ & 50 \\
\hline Controls & $43.3 \pm 35.9$ & 90 & $39.7 \pm 35.4$ & 51 & $36.3 \pm 35.5$ & 51 & $-3.4(-12.7 ; 5.8)$ & 51 \\
\hline Treatment effect & & & & & & & $-7.4(-22.0 ; 7.2)$ & \\
\hline \multicolumn{9}{|l|}{ Invalidity } \\
\hline NIV & $74.2 \pm 31.2$ & 99 & $70.6 \pm 34.0$ & 50 & $69.5 \pm 34.8$ & 50 & $-1.1(-9.4 ; 7.2)$ & 50 \\
\hline Controls & $68.7 \pm 33.5$ & 90 & $59.2 \pm 37.7$ & 51 & $57.3 \pm 39.4$ & 51 & $-2.0(-10.0 ; 6.1)$ & 51 \\
\hline Treatment effect & & & & & & & $0.9(-10.6 ; 12.3)$ & \\
\hline
\end{tabular}

Data presented as means ( \pm standard deviation) and changes ( $95 \%$ confidence intervals). ITT, intention-totreat; MRF-28, Maugeri Respiratory Questionnaire-28; n, numbers for analysis; NIV, non-invasive ventilation. " $p<0.05$ significant difference in change after 12 months within the group. ${ }^{*} p<0.05$ significant difference in change after 12 months between both groups (treatment effect). Low MRF-28 scores indicate high quality of life. A negative treatment effect signifies a bigger improvement after 12 months for the NIV group compared to standard treatment. 
Table S7.5 Changes in the Clinical Respiratory Questionnaire (CRQ) sub domains.

\begin{tabular}{|c|c|c|c|c|c|c|c|c|}
\hline & $\begin{array}{c}\text { ITT } \\
\text { Baseline } \\
\end{array}$ & $\mathbf{n}$ & $\begin{array}{c}\text { Completers } \\
\text { Baseline }\end{array}$ & $\mathbf{n}$ & $\begin{array}{l}\text { Completers } \\
12 \text { months }\end{array}$ & $\mathbf{n}$ & $\begin{array}{c}\text { Change score } \\
12 \text { months }\end{array}$ & $\mathbf{n}$ \\
\hline \multicolumn{9}{|l|}{ CRQ-Total } \\
\hline NIV & $3.5 \pm 1.1$ & 100 & $3.5 \pm 1.1$ & 50 & $4.2 \pm 1.2$ & 50 & $0.7(0.4 ; 1.1)^{n}$ & 50 \\
\hline Controls & $3.6 \pm 1.1$ & 89 & $3.6 \pm 1.1$ & 50 & $4.4 \pm 1.2$ & 50 & $0.7(0.4 ; 1.0)^{9}$ & 50 \\
\hline Treatment effect & & & & & & & $0.01(-0.41 ; 0.43)$ & \\
\hline \multicolumn{9}{|l|}{ Dyspnoea } \\
\hline NIV & $3.0 \pm 2.7$ & 100 & $3.1 \pm 1.6$ & 49 & $3.5 \pm 1.7$ & 49 & $0.4(0.03 ; 0.84)^{9}$ & 49 \\
\hline Controls & $3.25 \pm 1.4$ & 85 & $3.4 \pm 1.5$ & 46 & $3.9 \pm 1.5$ & 46 & $0.5(-0.002 ; 0.91)$ & 46 \\
\hline Treatment effect & & & & & & & $-0.02(-0.61 ; 0.58)$ & \\
\hline \multicolumn{9}{|l|}{ Fatigue } \\
\hline NIV & $2.70 \pm 1.3$ & 100 & $2.8 \pm 1.3$ & 50 & $3.8 \pm 1.5$ & 50 & $1.0(0.5 ; 1.4)^{9}$ & 50 \\
\hline Controls & $2.62 \pm 1.2$ & 89 & $2.5 \pm 1.0$ & 50 & $3.7 \pm 1.3$ & 50 & $1.1(0.8 ; 1.5)^{9}$ & 50 \\
\hline Treatment effect & & & & & & & $-0.18(-0.74 ; 0.39)$ & \\
\hline \multicolumn{9}{|l|}{ Emotion } \\
\hline NIV & $3.93 \pm 1.3$ & 100 & $3.9 \pm 1.3$ & & $4.6 \pm 1.3$ & 50 & $0.7(0.3 ; 1.1)^{9}$ & 50 \\
\hline Controls & $4.09 \pm 1.2$ & 89 & $4.1 \pm 1.3$ & 50 & $4.7 \pm 1.4$ & 50 & $0.6(0.3 ; 0.9)^{9}$ & 50 \\
\hline Treatment effect & & & & & & & $0.13(-0.37 ; 0.64)$ & \\
\hline \multicolumn{9}{|l|}{ Mastery } \\
\hline NIV & $3.86 \pm 1.4$ & 100 & $3.8 \pm 1.4$ & 50 & $4.6 \pm 1.4$ & 50 & $0.8(0.4 ; 1.2)^{9}$ & 50 \\
\hline Controls & $4.01 \pm 1.4$ & 89 & $4.0 \pm 1.4$ & 50 & $5.0 \pm 1.4$ & 50 & $0.9(0.5 ; 1.3)^{9}$ & 50 \\
\hline Treatment effect & & & & & & & $-0.13(-0.67 ; 0.40)$ & \\
\hline
\end{tabular}

Data presented as means ( \pm standard deviation) and changes ( $95 \%$ confidence intervals). CRQ, Clinical Respiratory Questionnaire; ITT, intention-to-treat; $n$, numbers for analysis; NIV, non-invasive ventilation. " $\mathrm{p}<0.05$ significant difference in change after 12 months within the group. ${ }^{*} p<0.05$ significant difference in change after 12 months between both groups (treatment effect). High CRQ scores indicate high quality of life. A positive treatment effect signifies a bigger improvement after 12 months for the NIV group compared to standard treatment. 
Table S7.6 Changes in the Severe Respiratory Insufficiency questionnaire (SRI) sub domains.

\begin{tabular}{|c|c|c|c|c|c|c|c|c|}
\hline & $\begin{array}{c}\text { ITT } \\
\text { Baseline }\end{array}$ & $\mathbf{n}$ & $\begin{array}{c}\text { Completers } \\
\text { Baseline } \\
\end{array}$ & $\mathrm{n}$ & $\begin{array}{l}\text { Completers } \\
12 \text { months }\end{array}$ & $n$ & $\begin{array}{c}\text { Change score } \\
12 \text { months } \\
\end{array}$ & $n$ \\
\hline \multicolumn{9}{|l|}{ SRI-Total } \\
\hline NIV & $48.1 \pm 15.0$ & 100 & $47.9 \pm 15.1$ & 50 & $55.0 \pm 15.4$ & 50 & $7.0(3.4 ; 10.7)^{9}$ & 50 \\
\hline Controls & $51.3 \pm 15.9$ & 90 & $53.6 \pm 16.9$ & 51 & $55.8 \pm 16.3$ & 51 & $2.2(-1.2 ; 5.6)$ & 51 \\
\hline Treatment effect & & & & & & & $4.8(-0.1 ; 9.7)$ & \\
\hline \multicolumn{9}{|l|}{ Respiratory complaints } \\
\hline NIV & $47.9 \pm 19.6$ & 100 & $48.9 \pm 21.2$ & 50 & $55.6 \pm 19.4$ & 50 & $6.7(2.1 ; 11.2)^{9}$ & 50 \\
\hline Controls & $44.0 \pm 18.6$ & 90 & $45.7 \pm 18.5$ & 51 & $54.3 \pm 16.7$ & 51 & $8.6(3.8 ; 13.5)^{9}$ & 51 \\
\hline Treatment effect & & & & & & & $-2.0(-8.6 ; 4.6)$ & \\
\hline \multicolumn{9}{|l|}{ Physical functioning } \\
\hline NIV & $32.0 \pm 18.5$ & 100 & $32.7 \pm 21.6$ & 50 & $36.5 \pm 21.6$ & 50 & $3.8(-1.4 ; 9.0)$ & 50 \\
\hline Controls & $32.5 \pm 21.9$ & 90 & $37.3 \pm 21.4$ & 51 & $41.3 \pm 21.6$ & 51 & $4.1(-1.7 ; 9.9)$ & 51 \\
\hline Treatment effect & & & & & & & $0.29(-8.0 ; 7.4)$ & \\
\hline \multicolumn{9}{|l|}{ Attendant symp+sleep } \\
\hline NIV & $57.1 \pm 18.4$ & 100 & $54.4 \pm 17.9$ & 50 & $64.8 \pm 18.0$ & 50 & $10.4(5.3 ; 15.5)^{9}$ & 50 \\
\hline Controls & $58.6 \pm 20.0$ & 90 & $59.9 \pm 22.3$ & 51 & $61.7 \pm 18.4$ & 51 & $1.7(-2.9 ; 6.4)$ & 51 \\
\hline Treatment effect & & & & & & & $8.7(1.9 ; 15.4)^{*}$ & \\
\hline \multicolumn{9}{|l|}{ Social relationships } \\
\hline NIV & $58.3 \pm 17.1$ & 100 & $56.3 \pm 14.0$ & 50 & $60.9 \pm 18.9$ & 50 & $4.6(-0.2 ; 9.4)$ & 50 \\
\hline Controls & $66.5 \pm 17.1$ & 90 & $68.2 \pm 17.3$ & 51 & $64.3 \pm 17.4$ & 51 & $-3.9(-7.7 ; 0.03)$ & 51 \\
\hline Treatment effect & & & & & & & $8.4(2.4 ; 14.5)^{*}$ & \\
\hline \multicolumn{9}{|l|}{ Anxiety } \\
\hline NIV & $46.2 \pm 22.5$ & 100 & $47.0 \pm 24.6$ & 50 & $59.8 \pm 22.1$ & 52 & $12.8(7.1 ; 18.5)^{9}$ & 50 \\
\hline Controls & $50.4 \pm 25.4$ & 90 & $52.6 \pm 26.3$ & 51 & $58.2 \pm 23.0$ & 51 & $5.7(0.6 ; 10.7)^{9}$ & 51 \\
\hline Treatment effect & & & & & & & $7.1(-0.4 ; 14.6)$ & \\
\hline \multicolumn{9}{|l|}{ Well-being } \\
\hline NIV & $52.6 \pm 20.1$ & 100 & $52.2 \pm 19.1$ & 50 & $58.1 \pm 22.9$ & 50 & $5.9(0.7 ; 11.1)^{9}$ & 50 \\
\hline Controls & $59.3 \pm 20.4$ & 90 & $60.4 \pm 22.1$ & 51 & $60.3 \pm 21.3$ & 51 & $-0.1(-4.7 ; 4.4)$ & 51 \\
\hline Treatment effect & & & & & & & $6.0(-0.8 ; 12.8)$ & \\
\hline \multicolumn{9}{|l|}{ Social functioning } \\
\hline NIV & $43.1 \pm 40.6$ & 100 & $44.0 \pm 19.4$ & 50 & $49.1 \pm 20.1$ & 50 & $5.1(-0.2 ; 10.4)$ & 50 \\
\hline Controls & $48.0 \pm 20.4$ & 90 & $51.4 \pm 21.6$ & 51 & $50.7 \pm 23.1$ & 51 & $-0.8(-5.9 ; 4.4)$ & 51 \\
\hline Treatment effect & & & & & & & $5.9(-1.4 ; 13.2)$ & \\
\hline
\end{tabular}

Data presented as means ( \pm standard deviation) and changes (95\% confidence intervals). Attendant symp+sleep, attendant symptoms and sleep sub domain; ITT, intention-to-treat; $n$, numbers for analysis; NIV, non-Invasive ventilation; SRI, Severe Respiratory Insufficiency questionnaire. " $p<0.05$ significant difference in change after 12 months within the group. ${ }^{*} p<0.05$ significant difference in change after 12 months between both groups (treatment effect). High SRI scores indicate high quality of life. A positive treatment effect signifies a bigger improvement after 12 months for the NIV group compared to standard treatment. 
Table S7.7 Changes in the Groningen Activity and Restriction Scale (GARS), Hospital Anxiety and Depression Scale (HADS) and Medical Research Council (MRC) sub domains.

\begin{tabular}{|c|c|c|c|c|c|c|c|c|}
\hline & $\begin{array}{l}\text { ITT } \\
\text { Baseline } \\
\end{array}$ & n & $\begin{array}{l}\text { Completers } \\
\text { Baseline }\end{array}$ & $\mathbf{n}$ & $\begin{array}{l}\text { Completers } \\
12 \text { months }\end{array}$ & $\mathbf{n}$ & $\begin{array}{l}\text { Change score } \\
12 \text { months }\end{array}$ & $\mathbf{n}$ \\
\hline \multicolumn{9}{|c|}{ GARS-Total } \\
\hline NIV & $36.7 \pm 7.5$ & 100 & $36.3 \pm 8.3$ & 50 & $34.6 \pm 9.4$ & 50 & $-1.6(-3.3 ; 0.1)$ & 50 \\
\hline Controls & $36.8 \pm 8.5$ & 90 & $34.7 \pm 1.2$ & 51 & $32.7 \pm 8.2$ & 51 & $-2.0(-4.1 ; 0.1)$ & 51 \\
\hline \multicolumn{2}{|c|}{ Treatment effect } & & & & & & $0.4(-2.3 ; 3.0)$ & \\
\hline \multicolumn{9}{|c|}{ HADS-Total } \\
\hline NIV & $15.9 \pm 9.2$ & 98 & $15.6 \pm 8.5$ & 48 & $13.9 \pm 8.8$ & 48 & $-1.7(-4.0 ; 0.6)$ & 48 \\
\hline Controls & $14.5 \pm 8.8$ & 87 & $13.1 \pm 9.1$ & 50 & $12.7 \pm 9.3$ & 50 & $-0.4(-2.2 ; 1.3)$ & 50 \\
\hline \multicolumn{2}{|c|}{ Treatment effect } & & & & & & $-1.3(-4.1 ; 1.6)$ & \\
\hline \multicolumn{9}{|c|}{ HADS anxiety } \\
\hline NIV & $7.8 \pm 5.0$ & 99 & $7.9 \pm 4.7$ & 49 & $6.6 \pm 4.7$ & 49 & $-1.3(-2.3 ;-0.2)^{9}$ & 49 \\
\hline Controls & $7.3 \pm 4.6$ & 88 & $6.8 \pm 4.8$ & 51 & $5.9 \pm 4.8$ & 51 & $-0.9(-1.8 ; 0.02)$ & 51 \\
\hline \multicolumn{2}{|c|}{ Treatment effect } & & & & & & $-0.4(-1.8 ; 1.0)$ & \\
\hline \multicolumn{9}{|c|}{ HADS depression } \\
\hline NIV & $8.1 \pm 4.9$ & 99 & $7.5 \pm 4.8$ & 50 & $7.1 \pm 4.6$ & 50 & $-0.4(-1.7 ; 0.9)$ & 50 \\
\hline Controls & $7.1 \pm 4.7$ & 87 & $6.3 \pm 4.7$ & 50 & $6.7 \pm 5.1$ & 50 & $0.4(-0.6 ; 1.5)$ & 50 \\
\hline \multicolumn{2}{|c|}{ Treatment effect } & & & & & & $-0.8(0.8 ;-2.5)$ & \\
\hline \multicolumn{9}{|l|}{ MRC } \\
\hline NIV & $3.9 \pm 1.0$ & 98 & $3.8 \pm 1.4$ & 49 & $3.5 \pm 1.4$ & 49 & $-0.37(-0.72 ;-0.02)^{9}$ & 49 \\
\hline Controls & $3.8 \pm 1.0$ & 90 & $3.6 \pm 1.1$ & 51 & $3.3 \pm 1.2$ & 51 & $-0.31(-0.71 ; 0.08)$ & 51 \\
\hline \multicolumn{2}{|c|}{ Treatment effect } & & & & & & $-0.05(-0.58 ; 0.47)$ & \\
\hline
\end{tabular}

Data presented as means ( \pm standard deviation) and changes ( $95 \%$ confidence intervals). GARS, Groninger Activity Restriction Scale; HADS, Hospital Anxiety and Depression Scale; ITT, intention-to-treat; $n$, numbers for analysis; NIV, non-invasive ventilation; MRC, Medical Research Council dyspnoea scale. " $p<0.05$ significant difference in change after 12 months within the group. ${ }^{*} \mathrm{p}<0.05$ significant difference in change after 12 months between both groups (treatment effect). Low GARS scores indicate better daily activity levels. Low HADS scores indicate better mood state. Lower MRC scores represent less dyspnoea. A negative treatment effect signifies a bigger improvement after 12 months for the NIV group compared to standard treatment. 


\section{References}

1. Plant, P.K., J.L. Owen, and M.W. Elliott, One year period prevalence study of respiratory acidosis in acute exacerbations of COPD: implications for the provision of non-invasive ventilation and oxygen administration. Thorax, 2000. 55(7): p. 550-4.

2. Bott, J., et al., Randomised controlled trial of nasal ventilation in acute ventilatory failure due to chronic obstructive airways disease. Lancet, 1993. 341(8860): p. 1555-7.

3. Brochard, L., et al., Noninvasive ventilation for acute exacerbations of chronic obstructive pulmonary disease. N Engl J Med, 1995. 333(13): p. 817-22.

4. Tuggey, J.M., P.K. Plant, and M.W. Elliott, Domiciliary non-invasive ventilation for recurrent acidotic exacerbations of COPD: an economic analysis. Thorax, 2003. 58(10): p. 867-71.

5. Chu, C.M., et al., Readmission rates and life threatening events in COPD survivors treated with noninvasive ventilation for acute hypercapnic respiratory failure. Thorax, 2004. 59(12): p. 1020-5.

6. Cheung, A.P., et al., A pilot trial of non-invasive home ventilation after acidotic respiratory failure in chronic obstructive pulmonary disease. Int J Tuberc Lung Dis, 2010. 14(5): p. 642-9.

7. Gay, P.C., R.D. Hubmayr, and R.W. Stroetz, Efficacy of nocturnal nasal ventilation in stable, severe chronic obstructive pulmonary disease during a 3-month controlled trial. Mayo Clin Proc, 1996. 71(6): p. 533-42.

8. Meecham Jones, D.J., et al., Nasal pressure support ventilation plus oxygen compared with oxygen therapy alone in hypercapnic COPD. Am J Respir Crit Care Med, 1995. 152(2): p. 538-44.

9. Sin, D.D., et al., Effects of nocturnal noninvasive mechanical ventilation on heart rate variability of patients with advanced COPD. Chest, 2007. 131(1): p. 156-63.

10. Strumpf, D.A., et al., Nocturnal positive-pressure ventilation via nasal mask in patients with severe chronic obstructive pulmonary disease. Am Rev Respir Dis, 1991. 144(6): p. 1234-9.

11. Struik, F.M., et al., Nocturnal non-invasive positive pressure ventilation for stable chronic obstructive pulmonary disease. Cochrane Database Syst Rev, 2013(6): p. CD002878.

12. Clini, E., et al., The Italian multicentre study on noninvasive ventilation in chronic obstructive pulmonary disease patients. Eur Respir J, 2002. 20(3): p. 529-38.

13. McEvoy, R.D., et al., Nocturnal non-invasive nasal ventilation in stable hypercapnic COPD: a randomised controlled trial. Thorax, 2009. 64(7): p. 561-6.

14. Funk, G.C., et al., Long-term non-invasive ventilation in COPD after acute-on-chronic respiratory failure. Respir Med, 2011. 105(3): p. 427-34.

15. Vestbo, J., et al., Global strategy for the diagnosis, management, and prevention of chronic obstructive pulmonary disease: GOLD executive summary. Am J Respir Crit Care Med, 2013. 187(4): p. 347-65.

16. Kampelmacher, M.J., et al., [CBO guideline 'Oxygen therapy at home']. Ned Tijdschr Geneeskd, 2001. 145(41): p. 1975-80.

17. Cox, M., et al., Non-invasive monitoring of CO2 levels in patients using NIV for AECOPD. Thorax, 2006. 61(4): p. 363-4.

18. van der Molen, T., et al., Development, validity and responsiveness of the Clinical COPD Questionnaire. Health Qual Life Outcomes, 2003. 1: p. 13.

19. Carone, M., et al., Analysis of factors that characterize health impairment in patients with chronic respiratory failure. Quality of Life in Chronic Respiratory Failure Group. Eur Respir J, 1999. 13(6): p. 1293-300.

20. Guyatt, G., Measurement of health-related quality of life in chronic airflow limitation. Monaldi Arch Chest Dis, 1993. 48(5): p. 554-7.

21. Windisch, W., et al., The Severe Respiratory Insufficiency (SRI) Questionnaire: a specific measure of health-related quality of life in patients receiving home mechanical ventilation. J Clin Epidemiol, 2003. 56(8): p. 752-9.

22. Zigmond, A.S. and R.P. Snaith, The hospital anxiety and depression scale. Acta Psychiatr Scand, 1983. 67(6): p. 361-70.

23. Kempen, G.I., et al., The assessment of disability with the Groningen Activity Restriction Scale. Conceptual framework and psychometric properties. Soc Sci Med, 1996. 43(11): p. 1601-10. 
24. Bestall, J.C., et al., Usefulness of the Medical Research Council (MRC) dyspnoea scale as a measure of disability in patients with chronic obstructive pulmonary disease. Thorax, 1999. 54(7): p. 581-6.

25. Struik, F.M., et al., Nocturnal noninvasive positive pressure ventilation in stable COPD: a systematic review and individual patient data meta-analysis. Respir Med, 2014. 108(2): p. 329-37.

26. Echave-Sustaeta, J., et al., [Prognosis following acute exacerbation of COPD treated with non-invasive mechanical ventilation]. Arch Bronconeumol, 2010. 46(8): p. 405-10.

27. Murray, I., et al., Outcomes following non-invasive ventilation for hypercapnic exacerbations of chronic obstructive pulmonary disease. Thorax, 2011. 66(9): p. 825-6.

28. Struik, F.M., et al., The Severe Respiratory Insufficiency Questionnaire scored best in the assessment of health-related quality of life in chronic obstructive pulmonary disease. J Clin Epidemiol, 2013. 66(10): p. 1166-74.

29. Rodriguez-Roisin, R., Toward a consensus definition for COPD exacerbations. Chest, 2000. 117(5 Suppl 2): p. 398S-401S.

30. Miller, M.R., et al., Standardisation of spirometry. Eur Respir J, 2005. 26(2): p. 319-38.

31. Guyatt, G.H., et al., A measure of quality of life for clinical trials in chronic lung disease. Thorax, 1987. 42(10): p. 773-8. 


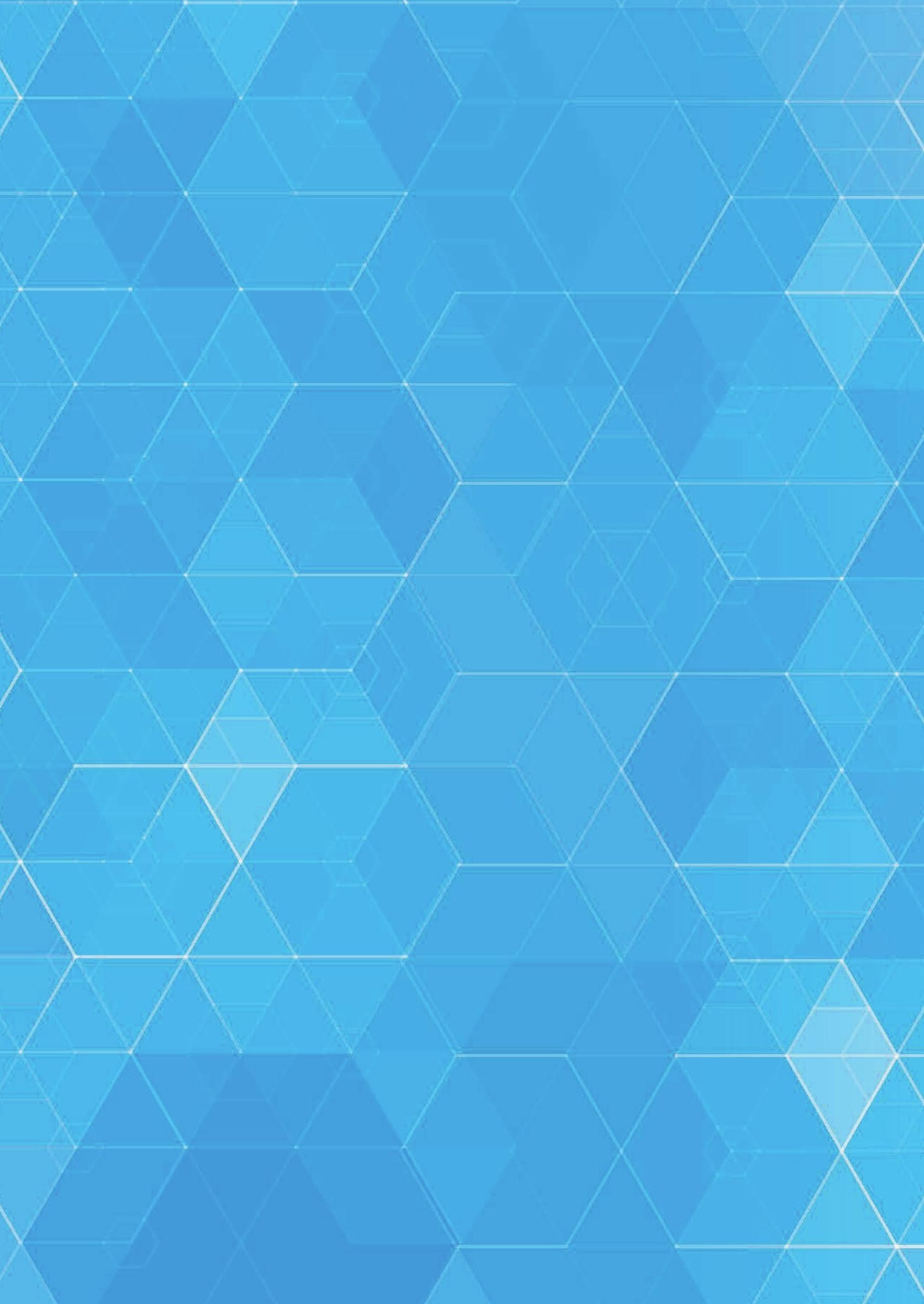




\title{
Chapter 8
}

\author{
Increased small intestinal
}

permeability during severe acute

exacerbation of COPD

Roy T. M. Sprooten, Kaatje Lenaerts, Dionne C.W. Braeken, Ilvy Grimbergen, Erica P. Rutten, Emiel F.M. Wouters, Gernot G.U. Rohde Published in Respiration 2018;95;334-342 


\section{Abstract}

\section{Background}

Disturbances of intestinal integrity, manifested by increased gastro-intestinal (GI) permeability, have been found in chronic obstructive pulmonary disease (COPD) patients during physical activity, often associated with intermittent hypoxic periods. Evidence about extra-pulmonary organ disturbances, especially of the GI tract, during hospitalized acute exacerbation of COPD (ECOPD) with hypoxaemic respiratory failure (RF) is lacking. The aim was to assess changes in GI permeability in patients with ECOPD and during recovery 4 weeks later.

\section{Methods}

All patients admitted to our hospital with ECOPD accompanied by hypoxaemia at admission $\left(\mathrm{PaO}_{2}<8.7 \mathrm{kPa}\right.$ or $\mathrm{O}_{2}$ saturation $\left.<93 \%\right)$ were screened between October 2013 and February 2014. Patients with a history of GI or renal disease, chronic heart failure, or use of non-steroidal anti-inflammatory drugs in the $48 \mathrm{~h}$ before the test were excluded. GI permeability was assessed by evaluating urinary excretion ratios of the orally ingested sugars lactulose/L-rhamnose (L/R ratio), sucrose/L-rhamnose (Su/R ratio) and sucralose/erythritol (S/E ratio).

\section{Results}

Seventeen patients with severe to very severe COPD completed the study. L/R ratio $\left(\times 10^{3}\right)$ at admission of ECOPD was significantly higher than in the recovery condition (40.9 [29.4--49.6] vs. 27.3 [19.5--47.7], $p=0.039$ ), indicating increased small intestinal permeability. There were no significant differences in the individual sugar levels in urine nor in the 0 - to 5 -h urinary S/E and Su/R ratios between the 2 visits.

\section{Conclusions}

This is the first study showing increased GI permeability during hospitalized ECOPD accompanied by hypoxaemic RF. Therefore, GI integrity in COPD patients is an attractive target for future research and for the development of interventions to alleviate the consequences of ECOPD. 


\section{Introduction}

Chronic obstructive pulmonary disease (COPD) is currently the fourth leading cause of death worldwide, and particularly emergency department visits, hospitalisations and readmissions for acute exacerbations are drivers of high disease burden and societal costs. ${ }^{1}$ Acute exacerbations of COPD (ECOPD) are associated with increased airway and systemic inflammation. ${ }^{2}$ During these acute episodes, gas exchange is often impaired, frequently resulting in acute respiratory failure (RF). Impaired gas exchange may be caused by increased airflow limitation, the rise in pulmonary vascular resistance, and increased mismatch of the ventilation/perfusion ratio. ${ }^{3-10}$ Although hypoxaemia is a frequent feature in patients admitted with severe ECOPD and oxygen therapy is considered as a standard treatment in hospitalised ECOPD, the extra-pulmonary systemic consequences of hypoxaemia are largely overlooked in clinical practice. ${ }^{8-12}$

Gastro-intestinal (GI) disturbance as a potential extra-pulmonary systemic consequence of hospitalised ECOPD accompanied by acute hypoxemic RF has been scarcely studied in COPD patients. Former studies in this group of patients about GI permeability have only been performed in stable disease states. ${ }^{13}$ Rutten et al. ${ }^{13}$ have found a difference in intestinal permeability between patients suffering from COPD and healthy controls. In their study, the execution of activities of daily living ( $A D L)$ enhanced this difference in intestinal permeability. In patients suffering from chronic heart failure, the intestinal morphology, permeability and absorption are modified and disturbed. ${ }^{14,15}$

Mucosal epithelial integrity is essential to prevent the entry of potentially harmful luminal particles, e.g., bacteria and their products, into the systemic circulation by means of transcellular and paracellular pathways. Disturbed intestinal integrity might lead to increased permeability of the intestinal mucosal barrier, which is one of the mechanisms promoting bacterial translocation, ${ }^{16,17}$ Bacterial translocation might also contribute to the observed acute chronic flare-up of systemic inflammation, which has been reported in ECOPD. ${ }^{2,18}$

Mechanistically, hypoxaemia might increase GI permeability. The enterocyte membranes, tight junctions, secreted mucus and immunologic factors form the intestinal barrier. The intestines are highly vascularized, and accordingly, the small gut villous epithelial cells are highly susceptible to oxygen shortage. ${ }^{14,15}$ This indicates that disturbances in the oxygen delivery to the intestinal mucosa could lead to disturbed intestinal integrity. Rutten et al. ${ }^{13}$ proposed that redistribution of blood flow from the splanchnic bed to the skeletal muscle tissue and to vital organs may lead to local tissue hypoxia, contributing to enterocyte damage and increased GI permeability in COPD patients during ADL, as described above. 
Moreover, it has been suggested that alterations in the $\mathrm{Gl}$ tract might also be associated with systemic inflammation. ${ }^{19,20}$ The epithelial apical junction complexes, consisting of tight junctions and adherence junctions, are, besides hypoxaemia, sensitive to circulating pro-inflammatory cytokines, which are present in higher levels during ECOPD. ${ }^{21}$ Although systemic inflammation is an important component in ECOPD, there is no evidence revealing a relationship with disturbances in the GI tract and systemic inflammation. $^{13}$

Based on the above-mentioned findings, disturbances of the intestinal integrity manifested by increased GI permeability might be present in patients with ECOPD complicated by severe hypoxaemic RF. Accordingly, we hypothesized that patients admitted for ECOPD accompanied by a hypoxaemic period show increased intestinal permeability.

\section{Methods}

\section{Subject inclusion}

This prospective study was approved by the ethical committee of the Maastricht University Medical Centre (MUMC; METC 13-2-040) and conducted in the MUMC, The Netherlands, according to the Declaration of Helsinki (59th WMA General Assembly, Seoul, October 2008) and Good Clinical Practice guidelines.

All patients admitted to the MUMC with ECOPD between October 2013 and February 2014 were screened according to the following inclusion criteria: clinical diagnosis of ECOPD according to the GOLD statement, hypoxaemia at admission $\left(\mathrm{PaO}_{2}<8.7 \mathrm{kPa}\right.$ or $\mathrm{O}_{2}$ saturation $<93 \%$ ) and a minimum age of 40 years. Patients with a history of $\mathrm{Gl}$ or renal disease, chronic heart failure and use of non-steroidal anti-inflammatory drugs in the $48 \mathrm{~h}$ before the test were excluded because these factors are known to influence $\mathrm{GI}$ permeability (inhibition of cyclooxygenases 1 and 2). ${ }^{13,22-24}$ Patients who could not be tested within the first $72 \mathrm{~h}$ after hospital admission, mostly due to logistic reasons, were excluded. Nineteen patients gave written informed consent.

\section{Study design}

The study consisted of 2 visits, the first shortly after admission to the hospital (visit 1 ) and the second visit during recovery, 4 weeks after the first test day (visit 2). Recovery was defined as regression of symptoms back to pre-existing levels, absence of additional oxygen or return to pre-existing long-term oxygen levels and normal vital 
signs. Because of uncertainty of recovery duration after ECOPD, the follow-up visit was planned 4 weeks after the first visit (first test day). ${ }^{25-28}$ During the second visit, regression of symptoms was assessed by the treating physician. In case of a readmission because of an ECOPD, visit 2 would take place 4 weeks after discharge of this episode. The same study procedures took place at both visits. The study did not interfere with the standard medical care of the exacerbation.

\section{Study procedures}

After overnight fast (24:00-07:00), a sugar solution (details see below) was orally ingested after collection of a baseline blood and urine sample. Before ingesting the sugar mixture, the saturation was measured using a pulse oximeter. Arterial blood gases were only collected for standard medical care. Until $1 \mathrm{~h}$ before the intake of the sugar solution, subjects were allowed to drink water ad libitum. Thereafter, subjects were allowed to drink a maximum of $500 \mathrm{~mL}$ water per hour. Medication intake was permitted. One hour after intake of the sugar solution, a standardized breakfast was served. Urine was collected during $5 \mathrm{~h}$ after drinking the sugar mixture. Patients were not allowed to eat after breakfast until the end of the test ( $4 \mathrm{~h}$ after breakfast).

\section{Assessment of Gl permeability}

The differential sugar absorption assays for gut permeability are based on orally ingested inert sugars that differentially cross the intestinal barrier based on their size. ${ }^{29}$ Large sugars (oligosaccharides) typically cross the intestinal barrier paracellularly into the circulation in case of disturbed integrity, small sugars (monosaccharides) also cross the barrier in normal state via the transcellular route and are used to correct for, e.g., differences in gastric emptying, intestinal transit and renal function. Hence, if there is increased leakage due to barrier loss, especially the large sugars cross the intestinal barrier into the circulation, leading to a higher oligosaccharide/monosaccharide ratio. These sugars are rapidly cleared by the kidney and can be detected in the Urine. ${ }^{16}$

In this study, intestinal permeability was assessed using a validated multi-sugar test. ${ }^{24,30}$ The sugar mixture contained $1 \mathrm{~g}$ lactulose (Centrafarm BV, Etten-Leur, The Netherlands), $1 \mathrm{~g}$ sucralose (Brenntag AG, Mülheim, Germany), 0.5 g L-rhamnose (Danisco A/S, Copenhagen, Denmark), 1 g erythritol (Cargill Europe, Mechelen, Belgium) and $1 \mathrm{~g}$ sucrose (Van Gilse, Suiker Unie, Dinteloord, The Netherlands) and was dissolved in $100 \mathrm{~mL}$ tap water just before administration. After ingesting the sugar solution, the subjects were allowed to drink another $200 \mathrm{~mL}$ water. Urine sugar concentrations were determined by isocratic ion exchange high-pressure liquid 
chromatography (Model PU-1980 pump; Jasco, Easton, MD, USA) with mass spectrometry (Model LTQ XL; Thermo Fisher Scientific, Waltham, MA, USA) as described by van Wijck et al.. ${ }^{24,30}$ The ratio of lactulose/L-rhamnose (L/R) and sucralose/erythritol (S/E) reflects intestinal permeability status. Lactulose and L-rhamnose are often used as a marker for small intestinal permeability, since the microbiota in the colon degrades lactulose and rhamnose. ${ }^{20,31}$ Sucralose and erythritol can serve as a marker for wholegut permeability, if collected over a 24 -h period, since they endure colonic bacterial fermentation. ${ }^{13,32,33}$

\section{Outcomes}

The primary outcome of this study was the change in GI permeability (L/R ratio, sucrose/L-rhamnose ratio [Su/R ratio] and S/E ratio) between admission and after recovery.

\section{Data collection}

Medical history, most recent lung function data (including forced expiratory volume in $1 \mathrm{~s}\left(\mathrm{FEV}_{1}\right)$, forced vital capacity (FVC), $\mathrm{FEV}_{1} / \mathrm{FVC}$ ratio, residual volume, total lung capacity and diffusion capacity), comorbidities using the Charlson Comorbidity Index ${ }^{34}$, medication, arterial blood gas, vital signs, physical examination at admission, full blood count, liver and renal function and inflammatory parameters (C-reactive protein (CRP) and white blood count) were assessed at admission. At both test days, vital signs, Borg score, medication and current symptoms were monitored.

\section{Statistical analysis}

Data analyses were performed using SPSS for MAC version 21.0. Because of the small population, data were considered to be not normally distributed and analysed using non-parametric tests. Continuous data are presented as median and first and third quartiles (25-75\% percentiles) and categorical data as percentages. Changes in L/R ratio between admission and recovery were calculated in absolute numbers and percentages. To compare characteristics which might be related to change in intestinal permeability between groups, the Wilcoxon signed-rank test was performed. A p value $<0.05$ was considered to be statistically significant.

The sample size was based on the outcome of a study in patients with chronic heart failure. ${ }^{15}$ In this study, the permeability index (lactulose/mannitol ratio) was used as a marker of intestinal permeability. Performing a power calculation with the formula 
$n=(\alpha+\beta)^{2} \times 2 \times \mathrm{SD}^{2} / \mathrm{d}^{2}$ and $\alpha=0.05, \beta=0.20$, the difference (d) in lactulose $/$ mannitol ratio $=0.35$ and $S D=4.0$, the amount of subjects needed was at least 16.3. Hence, 17 patients had to complete the study.

\section{Results}

\section{Subject selection}

From October 2013 until February 2014, 106 patients admitted for an ECOPD were screened (Figure 8.1). Twenty-three patients did not meet to the inclusion criteria for the following reasons: absence of hypoxaemia $(n=18,78.3 \%)$, not able to give informed consent $(n=4,17.4 \%)$ and age $<40$ years $(n=1,4.3 \%)$. Forty-two patients were excluded because of renal failure $(n=21,50.0 \%)$, heart failure $(n=4,9.5 \%)$, renal and heart failure ( $n=2,4.8 \%)$, acute $\mathrm{Gl}$ complaints $(n=2,4.8 \%)$, not being able to be tested within $72 \mathrm{~h}$ $(n=12,28.6 \%)$ and urinary incontinence $(n=1,2.4 \%)$. Fourteen had a readmission and had already been screened during an earlier admission. From the remaining 27 patients, 8 patients declined to participate. In total, 19 patients were included into the study. After the first visit, 2 patients withdrew from the study and did not participate in the second visit.

Figure 8.1 Flowchart of the subject selection. E-COPD, acute exacerbation of chronic obstructive pulmonary disease.

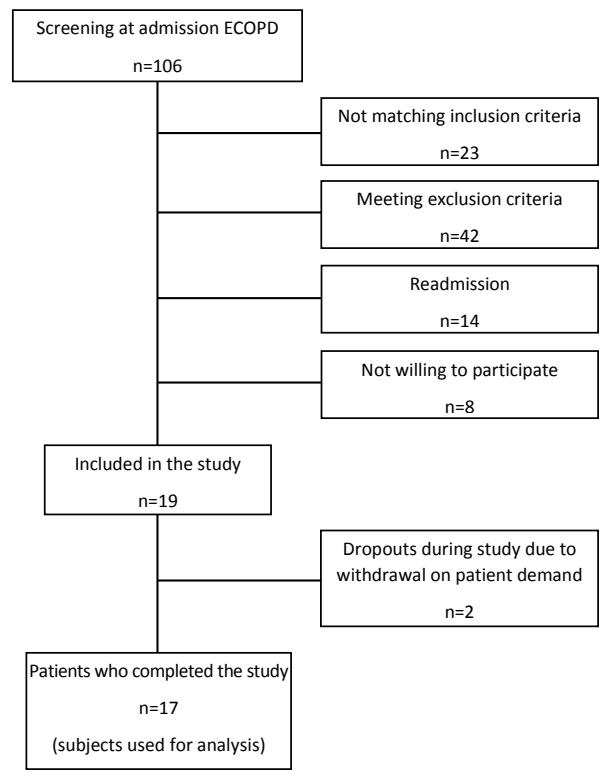


At admission, all patients were hypoxaemic (initial $\mathrm{SpO}_{2} 84 \%$ [80--89\%]) at presentation to the emergency room (Table 8.2). All patients needed oxygen treatment at the emergency room. Seven patients were hypercapnic, and 2 patients required noninvasive ventilation because of acute respiratory acidosis. In 10 patients, antibiotic treatment was initiated, and 3 patients had a consolidation on chest X-ray. In 4 patients, the CRP level was higher than $50 \mathrm{mg} / \mathrm{L}$. The median length of hospital stay was 6 days.

Table 8.2 Clinical characteristics at admission for ECOPD $(n=17)$.

\begin{tabular}{lc}
\hline Initial $\mathrm{SpO}_{2}, \%$ & $84(80-89)$ \\
Oxygen need at ER & $17(100)$ \\
$\mathrm{ABG}$ at admission & $7.4(6.8-8.3)$ \\
$\mathrm{pO}_{2}, \mathrm{kPa}$ & $5.7(5.0-7.8)$ \\
$\mathrm{pCO}_{2}, \mathrm{kPa}$ & $7.39(7.34-7.45)$ \\
$\mathrm{pH}$ & $27.1(23.9-32.6)$ \\
$\mathrm{HCO}_{3}{ }^{-}, \mathrm{mmol} / \mathrm{L}$ & $2.1(0.2-5.3)$ \\
$\mathrm{BE}$ & $7(41)$ \\
$\mathrm{pCO}_{2} \geq 6.0, \mathrm{mmol} / \mathrm{L}$ & $88(70-96)$ \\
$\mathrm{SpO}_{2}$ at admission after stabilizing, \% & $3(18)$ \\
$\mathrm{Pneumonia}$ and AE-COPD & $4(24)$ \\
$\mathrm{CRP}>50 \mathrm{mg} / \mathrm{L}$ & $2(12)$ \\
$\mathrm{NIV}$ need & $10(59)$ \\
Antibiotics started at admission & $6.0(3-9)$ \\
LOS, days &
\end{tabular}

Data are presented as medians and $25-75 \%$ percentiles or $\mathrm{n}(\%)$. Hypoxia criteria were $\mathrm{pO}_{2}<8.7 \mathrm{kPa}$ or $\mathrm{SpO}$ at admission $\leq 93 \%$. E-COPD, acute exacerbation of chronic obstructive pulmonary disease; $\mathrm{SpO}_{2}$, oxygen saturation; ER, emergency room; $A B G$, arterial blood gas; $B E$, base excess; CRP, C-reactive protein; NIV, noninvasive positive pressure ventilation; LOS, length of hospital stay.

\section{Clinical parameters at test days}

The clinical parameters at visit 1 and 2 are shown in Table 8.3. The oxygen saturation at visit 1 improved compared to the measurements at admission $\left(\mathrm{SpO}_{2}\right.$ at admission, $88 \%$ [86-93\%] vs. $\mathrm{SpO}_{2}$ at visit 1, 93\% [91.5-95\%], $\left.\mathrm{p}=0.005\right)$. Unfortunately, due to ethical considerations according to the approved protocol, we were not able to test patients directly at the time of admission when hypoxaemia was most prominent and, likely, the intestinal barrier damage was most severe. We did our best to schedule the tests as quickly as possible, which led to an interval time from admission until first visit of $52 \mathrm{~h}$ (28-69 h). After discharge, the second test day was scheduled. The median time between visit 1 and visit 2 was 34 days (28-90). Three patients had a readmission due to ECOPD, and 1 patient had an ECOPD but was not admitted during the follow-up. The second test day in these patients was postponed until the patient was in a stable 
condition. All patients were investigated according to the study protocol and were stable at the second visit.

Table 8.3 Clinical parameters at test days (visit 1 and 2) ( $n=17)$.

\begin{tabular}{lccc}
\hline & Visit 1 & Visit 2 & p-value* \\
\hline $\mathrm{SpO}_{2}, \%$ & $93(91.5-95)$ & $95(91.5-97)$ & 0.072 \\
Pulse, /min & $80(70.5-92)$ & $73(67.5-90)$ & 0.343 \\
$\mathrm{RR}, / \mathrm{min}$ & $14(13.5-19)$ & $14(12-16.5)$ & 0.132 \\
Systolic BP, mm Hg & $123(118-137)$ & $132(119-137.5)$ & 0.522 \\
Diastolic BP, mm Hg & $69(63-84.5)$ & $75(67.5-78.5)$ & 0.579 \\
Borg dyspnoea score & $7(4.5-7.5)$ & $3(1.5-3.5)$ & 0.001 \\
Oxygen amount, L/min & $1(0-5)$ & $0.6(0-4)$ & 0.116 \\
\hline
\end{tabular}

Data are presented as medians and $25-75 \%$ percentiles. $\mathrm{SpO}_{2}$, oxygen saturation; RR, respiratory rate; $\mathrm{BP}$, blood pressure. * Wilcoxon signed-rank test.

The clinical parameters at visit 2 improved, including oxygen saturation (median $\mathrm{SpO}_{2}$ 95\% [91.5-97\%]), and clinical symptoms were within a normal range. The Borg dyspnoea score at visit 2 improved significantly compared to visit 1 (3 [1-5] vs. 7 [2-8], $\mathrm{p}=0.001$ ). $\mathrm{SpO}_{2}$ was significantly improved at visit 2 compared to the situation at admission (median $95 \%$ vs. $88 \%, p=0.006$ ), and there was a trend to significant improvement of $\mathrm{SpO}_{2}$ at visit 2 if compared to visit $1(p=0.072)$.

\section{Gl permeability}

The 0 - to 5-h urinary L/R ratio, reflecting small intestine permeability, was significantly increased at visit 1 (ECOPD visit during hospital stay) compared to visit 2 (the stable condition at home) (Table 8.4). Figure 8.2a shows the individual values of the 0 - to 5 -h urinary $L / R$ ratio and the change between both visits. The median absolute difference in the 0 - to 5 -h urinary L/R ratio $\left(\times 10^{3}\right)$ in our study group was -13.67 ( -57.15 to 66.48$)$, and the relative change was $-35.5 \%(-55.2$ to $180.2 \%)$. In 3 patients, the L/R ratio was elevated at the stable condition (visit 2; Figure 8.2a). The absolute delta of the 0 - to 5-h urinary L/R ratio $\left(\times 10^{3}\right)$ in these patients was, respectively, $0.73(2.7 \%), 66.48(180.2 \%)$ and 35.78 (90.0\%). The first and second patient had hypercapnia at admission. The first patient had a readmission and the second visit was performed after 42 days. The second and third patient had a short hospital admission and no readmission, respectively. Their deviant $L / R$ ratio change over time could not be explained based on other clinical parameters.

The 0 - to 5-h urinary S/E ratio, including the permeability of the proximal colon, was similar in both visits. Figure $8.2 \mathrm{~b}$ shows the individual values and the trend of the 0 - to 
5-h urinary S/E ratio at the first and second visit. The median absolute difference in the 0 - to 5 -h urinary S/E ratio $\left(\times 10^{3}\right)$ in our study group was $-0.13(-105.8$ to 55.61$)$, and the relative change was $-1.6 \%(-86.4$ to $92.6 \%)$. The anticipated decrease in the 0 - to $5-h$ urinary S/E ratio between both visits was not observed in 8 pat Figure $8.2 \mathrm{~b}$ ). In 1 patient, the measurement of sucralose and erythritol concentrations in urine failed during the last visit.

Table 8.4 Gastro-intestinal permeability during ECOPD compared to stable condition ( $n=17)$.

\begin{tabular}{lccc}
\hline & Visit 1 & Visit 2 & p-value* \\
\hline Lactulose & $29.1(16.9-43.8)$ & $20.3(10.8-48.8)$ & 0.332 \\
L-Rhamnose & $795.8(400.3-1,061.1)$ & $566.5(383-1,679)$ & 0.586 \\
Erythritol & $10,454(6,014-13,242)$ & $5,549(3,768-14,491)$ & 0.266 \\
Sucrose & $50.4(32.6-122.7)$ & $75.7(22.2-122.3)$ & 0.687 \\
Sucralose & $104.5(55.9-187.2)$ & $66.5(27.4-222.3)$ & 0.227 \\
L/R ratio, $\times 10^{3}$ & $40.9(29.4-49.6)$ & $27.3(19.5-47.7)$ & 0.039 \\
S/E ratio, $\times 10^{3}$ & $11.2(8.2-16.8)$ & $10.5(8.8-16.7)(n=16)$ & 1.00 \\
Su/R ratio, $\times 10^{3}$ & $77.7(44.0-159.7)$ & $86.2(42.1-190.6)$ & 0.906 \\
\hline
\end{tabular}

Data are presented as medians and $25-75 \%$ percentiles. The table shows the sugar urinary excretion levels after drinking a sugar solution measured during both visits (conditions). ECOPD, acute exacerbation of chronic obstructive pulmonary disease; L/R ratio, ratio between lactose and L-rhamnose; S/E ratio, ratio between sucralose and erythritol; Su/R ratio, ratio between sucrose and rhamnose. * Wilcoxon signed-rank test.

Figure 8.2 Gastrointestinal permeability during ECOPD (visit 1) compared to stable condition (visit 2) according to 0 - to 5-h urinary L/R ratio $\left(\times 10^{3}\right)(\mathbf{a})$, S/E ratio $\left(\times 10^{3}\right)(\mathbf{b})$, and Su/R ratio $\left(\times 10^{3}\right)(\mathbf{c})$.
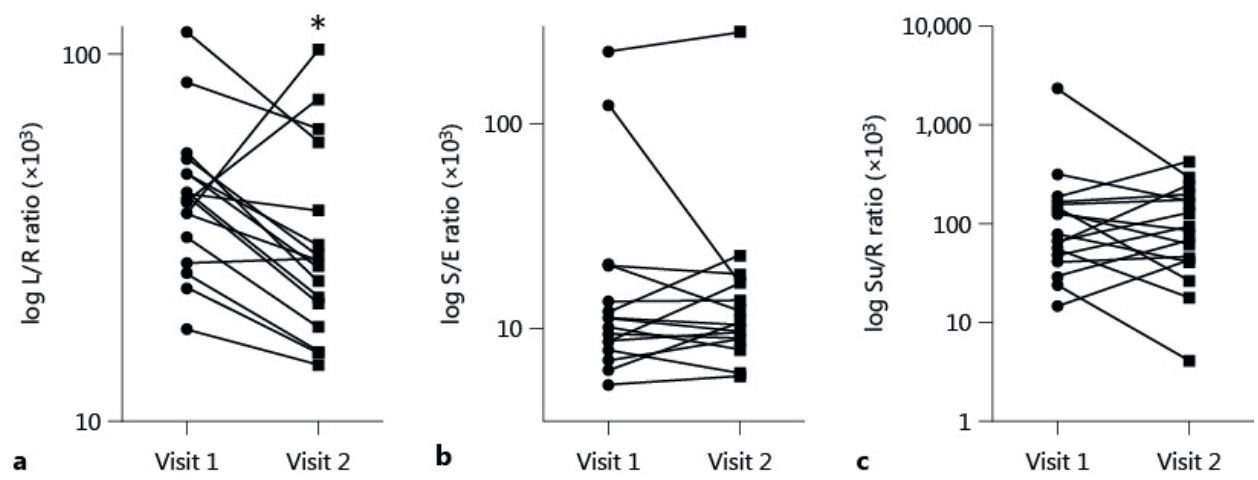

$* \mathrm{p}$ value $<0.05$. AE-COPD, acute exacerbation of chronic obstructive pulmonary disease; L/R ratio, lactulose/L-rhamnose ratio; S/E ratio, sucralose/erythritol ratio Su/R ratio, sucrose/L- rhamnose ratio. 
Also the Su/R ratio, reflecting gastric/duodenal permeability, was not different between both visits. The individual values and the trend of the 0 - to 5-h urinary Su/R ratio at the first and second visit are shown in Figure 8.2c. The median absolute difference in the 0to 5 -h urinary $\mathrm{Su} / \mathrm{R}$ ratio $\left(\times 10^{3}\right)$ in our study group was $4.43(-2,051.27$ to 231.23$)$, and the relative change was $10.8 \%$ ( -87.7 to $281.4 \%)$. In 8 patients, a negative trend (delta) in the 0 - to 5 -h urinary $\mathrm{Su} / \mathrm{R}$ ratio was found.

There were no significant differences in the individual sugar levels in urine between the 2 visits (Table 8.4).

\section{Discussion}

To our knowledge, this is the first study showing increased small intestinal permeability, reflected by a significantly higher 0 - to 5 -h urinary L/R ratio, during hospitalised ECOPD complicated by severe hypoxaemic RF compared to a clinically stable condition (4 weeks later). Increased intestinal permeability has already been described in various other extra-intestinal diseases and suggested to contribute to pathology. Sandek et al. ${ }^{15}$ reported morphological and functional alterations of the gut in CHF patients, with an increased permeability index in both the small and large intestine. Pijls et al. ${ }^{35}$ reported an increased colonic permeability in patients with compensated liver cirrhosis. In patients with burn injuries, in patients undergoing cardiopulmonary bypass surgery and in critically ill patients who developed multi-organ failure, intestinal permeability was shown to be increased. ${ }^{36-40}$ Interestingly, also studies performed in healthy athletes suggest that strenuous exercise limits oxygen supply to the GI tract, which has a negative impact on $\mathrm{Gl}$ barrier function. ${ }^{41,42}$ Oktedalen et al. ${ }^{43}$ showed increased intestinal permeability after marathon running, and higher intestinal permeability was observed in runners with GI symptoms compared to asymptomatic runners. ${ }^{44}$

The first study investigating GI permeability in COPD patients in a stable conditions has been published by Rutten et al.. ${ }^{13}$ In that study, intestinal permeability was higher in patients suffering from COPD compared to healthy controls. In addition, the execution of an ADL test augmented the differences in intestinal permeability. Importantly, they found significantly higher plasma intestinal fatty acid-binding protein levels after performing this ADL test in stable COPD patients and not in healthy controls, suggesting the occurrence of enterocyte damage specifically in COPD patients. ${ }^{13}$ Data from that study suggested splanchnic oxygen shortage to develop during physical activity in COPD patients and led us to conduct the present study in ECOPD, in which hypoxaemia is an important manifestation. 
Manifestations in the GI tract have not yet been investigated in patients hospitalised for ECOPD suffering from hypoxaemia at the time of admission. Our results revealed an increased intestinal permeability in patients undergoing an ECOPD compared to the same patients in a stable condition of COPD. In our pilot study, we observed a significant difference specifically for the urinary L/R ratio and not for the other ratios. This might be explained by the fact that the L/R ratio typically reflects permeability of the small intestine, which is more susceptible to ischaemic injury than the colon. ${ }^{45}$ This is supported by the finding that the 0 to 5 -h urinary S/E ratio, including the permeability of the proximal colon, was similar at both visits. Importantly, we tried to shorten the time window between the hypoxaemic event - overtly present in all patients at admission - and measurement of intestinal permeability as much as possible. For ethical reasons, we were, however, not able to perform the measurements directly at admission. This could imply that we did not capture the intestinal leakiness at its maximal level, as the recovery phase might have been already initiated at visit 1 , as also objectified by improved hypoxaemia compared to hypoxaemia at admission. However, a tendency for worse $\mathrm{SpO}_{2}$ at visit 1 compared to the stable situation at visit 2 was observed.

Although our study was not designed to investigate the underlying mechanism of intestinal disturbances in ECOPD, we discuss potential routes which might underlie the increased intestinal permeability observed in patients undergoing an ECOPD with hypoxaemic RF. Severe hypoxaemia by itself might result in inadequate oxygen delivery to the intestinal mucosa causing tissue hypoxia, which contributes to disturbed intestinal mucosal barrier function. The oxygenation of the small intestinal villi is regulated by a countercurrent circulation in which oxygen diffuses through the mucosa to the tip of the villi where the oxygen tension is the lowest. In case of oxygen shortage, this leads to more pronounced hypoxia at the tips, which is associated with increased enterocyte damage and integrity loss. ${ }^{46,47}$ In addition, hypoxaemia is thought to evoke a redistribution of blood flow away from less critical organs, such as the intestine, to those vital to survival (e.g., heart, brain). A strong correlation was found between the level of hypoxaemia and the decrease in mesenteric blood flow in patients during bronchoscopy, a reflex sympathetic activation presumably prominent in this situation. ${ }^{48}$ Such an increase in sympathetic activity has also been demonstrated in congestive heart failure patients and leads to a redistribution of blood flow away from the splanchnic circulation. ${ }^{49}$ Diminished splanchnic perfusion strongly correlates with enterocyte damage and precedes increased intestinal permeability in exercising healthy athletes. $^{42}$ Similarly, in the present patient population, activation of the sympathetic nervous system by hypoxaemia could result in changes in bowel perfusion and 
subsequently contribute to the observed intestinal barrier loss. In stable COPD, it has been shown that an imbalance in auto-regulation of the cardiac rhythm leads to arrhythmias and that sympathetic activation is related to a higher rate of hospitalizations and mortality in COPD. ${ }^{50,51}$

Next to hypoxic damage, the intestinal barrier might be affected by inflammatory mediators, which are known to circulate during ECOPD. ${ }^{52}$ For instance, cytokines can lead to alterations in the structure of tight junctions that connect enterocytes, thereby resulting in enhanced paracellular permeability and barrier loss. ${ }^{18}$ Some COPD patients have already increased levels of systemic inflammation markers in a stable state. ${ }^{53}$ Systemic inflammation markers, mainly CRP levels, often increase during ECOPD. In our patients, a quarter had a high CRP level (>50 mg/L). However, further subgroup analysis was not possible in our limited dataset, and correlation analysis between the urinary sugar ratio and parameters of inflammatory and gas exchange remained negative (data not shown). In summary, during ECOPD, the intestinal barrier might be disturbed by hypoxic damage or inflammatory mediators, or a combination. However, future, more invasive studies need to investigate in more detail the underlying mechanisms for increased intestinal permeability in ECOPD.

Intestinal disturbances can have several negative consequences. As mentioned above, inflammatory mediators in the circulation can affect the intestinal barrier. However, it has been shown that an increase in intestinal permeability also leads to an increase in bacterial translocation and systemic inflammation ${ }^{19}$, provoking a vicious circle of inflammation. Next, hypoxic enterocyte damage and consequent loss of enterocyte mass may disturb the digestion and absorption of nutrients. Another possible clinical impact of the increased intestinal permeability due to hypoxia in ECOPD is the decrease of effectiveness of oral medication. The uptake of medication may be disturbed leading to changes in pharmacokinetics and pharmacodynamics, which additionally may influence recovery from exacerbations.

Despite these findings, our study had some limitations. The number of patients that participated in this study was small; however, the power calculation indicated that we only needed 17 patients. In some of our patients with ECOPD, consolidation on chest $X$-ray was found, which is not unusual in these patients. One may ask if this could have influenced our results. These patients did not differ in outcome parameters compared to patients without consolidation. These patients, but also some of the patients without consolidation, received antibiotics at admission. In this cohort, we did not find evidence that pneumonia itself could have led to Gl disturbances. Some patients received 
antibiotics at admission. Gl side effects occur during the use of antibiotics; however, in a recent placebo-controlled human study, it was shown that 7-day antibiotics use did not affect intestinal barrier function. ${ }^{54}$ Blood gas analysis at the second test day would have supported our understanding of the patients' condition. However, in our setting, we used pulse oximetry to assess the patients' oxygenation, and it has been shown that pulse oximetry is an accurate measure in this setting. ${ }^{55}$ Patients were considered stable after 4 weeks, but it might be possible that patients were not yet fully recovered. We did, however, thoroughly interview all patients about recovery of all exacerbationrelated symptoms, and clinically, they were all fully recovered. Another possible limitation may be that we have not assessed cardiac status during exacerbation and after recovery as a contributing factor to impaired tissue oxygen delivery. However, patients did not show any clinical signs of acute heart failure.

\section{Conclusions}

In conclusion, this is the first study to demonstrate disturbances in intestinal permeability in patients with hospitalised ECOPD compared to stable COPD after a 4-week recovery period. Although the hypoxaemic event itself seems a likely explanation, the underlying mechanisms of our observations remain to be elucidated. This disturbed GI integrity in COPD patients is an attractive target for future research but also the development of new interventions to alleviate the consequences of ECOPD. 


\section{References}

1. Wedzicha, J.A. and T.A. Seemungal, COPD exacerbations: defining their cause and prevention. Lancet, 2007. 370(9589): p. 786-96.

2. van Eeden, S.F. and D.D. Sin, Chronic obstructive pulmonary disease: a chronic systemic inflammatory disease. Respiration, 2008. 75(2): p. 224-38.

3. Pauwels, R.A., et al., Global strategy for the diagnosis, management, and prevention of chronic obstructive pulmonary disease. NHLBI/WHO Global Initiative for Chronic Obstructive Lung Disease (GOLD) Workshop summary. Am J Respir Crit Care Med, 2001. 163(5): p. 1256-76.

4. Celli, B.R., W. MacNee, and A.E.T. Force, Standards for the diagnosis and treatment of patients with COPD: a summary of the ATS/ERS position paper. Eur Respir J, 2004. 23(6): p. 932-46.

5. John, B.A., S.G. Wood, and D.R. Hawkins, The pharmacokinetics and metabolism of sucralose in the mouse. Food Chem Toxicol, 2000. 38 Suppl 2: p. S107-10.

6. Wouters, E.F., Management of severe COPD. Lancet, 2004. 364(9437): p. 883-95.

7. GJ, G., Clinical tests of respiratory function. 2009.

8. Brill, S.E. and J.A. Wedzicha, Oxygen therapy in acute exacerbations of chronic obstructive pulmonary disease. Int J Chron Obstruct Pulmon Dis, 2014. 9: p. 1241-52.

9. Kent, B.D., P.D. Mitchell, and W.T. McNicholas, Hypoxemia in patients with COPD: cause, effects, and disease progression. Int J Chron Obstruct Pulmon Dis, 2011. 6: p. 199-208.

10. Kim, V., et al., Oxygen therapy in chronic obstructive pulmonary disease. Proc Am Thorac Soc, 2008. 5(4): p. 513-8.

11. Slenter, R.H., et al., Predictors of 1-year mortality at hospital admission for acute exacerbations of chronic obstructive pulmonary disease. Respiration, 2013. 85(1): p. 15-26.

12. Magnet, F.S., et al., Long-Term Oxygen Therapy: Comparison of the German and British Guidelines. Respiration, 2017. 93(4): p. 253-263.

13. Rutten, E.P.A., et al., Disturbed intestinal integrity in patients with COPD: effects of activities of daily living. Chest, 2014. 145(2): p. 245-252.

14. Krack, A., et al., The importance of the gastrointestinal system in the pathogenesis of heart failure. Eur Heart J, 2005. 26(22): p. 2368-74.

15. Sandek, A., et al., Altered intestinal function in patients with chronic heart failure. J Am Coll Cardiol, 2007. 50(16): p. 1561-9.

16. Bjarnason, I., A. MacPherson, and D. Hollander, Intestinal permeability: an overview. Gastroenterology, 1995. 108(5): p. 1566-81.

17. Berg, R.D., Bacterial translocation from the gastrointestinal tract. Trends Microbiol, 1995. 3(4): p. 14954.

18. Turner, J.R., Intestinal mucosal barrier function in health and disease. Nat Rev Immunol, 2009. 9(11): p. 799-809.

19. Hietbrink, F., et al., Systemic inflammation increases intestinal permeability during experimental human endotoxemia. Shock, 2009. 32(4): p. 374-8.

20. Hietbrink, F., et al., Pitfalls in gastrointestinal permeability measurement in ICU patients. Intensive Care Med, 2007. 33(12): p. 2216.

21. Bruewer, M., et al., Proinflammatory cytokines disrupt epithelial barrier function by apoptosisindependent mechanisms. J Immunol, 2003. 171(11): p. 6164-72.

22. Bours, M.J., et al., Effects of oral adenosine 5'-triphosphate and adenosine in enteric-coated capsules on indomethacin-induced permeability changes in the human small intestine: a randomized cross-over study. BMC Gastroenterol, 2007. 7: p. 23.

23. Sigthorsson, G., et al., COX-1 and 2, intestinal integrity, and pathogenesis of nonsteroidal antiinflammatory drug enteropathy in mice. Gastroenterology, 2002. 122(7): p. 1913-23.

24. van Wijck, K., et al., Novel multi-sugar assay for site-specific gastrointestinal permeability analysis: a randomized controlled crossover trial. Clin Nutr, 2013. 32(2): p. 245-51.

25. Seemungal, T.A., et al., Time course and recovery of exacerbations in patients with chronic obstructive pulmonary disease. Am J Respir Crit Care Med, 2000. 161(5): p. 1608-13. 
26. Wedzicha, J.A., Mechanisms of Chronic Obstructive Pulmonary Disease Exacerbations. Ann Am Thorac Soc, 2015. 12 Suppl 2: p. S157-9.

27. Pavord, I.D., et al., Exacerbations of COPD. Int J Chron Obstruct Pulmon Dis, 2016. 11 Spec Iss: p. 21-30.

28. Spencer, S., P.W. Jones, and G.S. Group, Time course of recovery of health status following an infective exacerbation of chronic bronchitis. Thorax, 2003. 58(7): p. 589-93.

29. Rooyakkers, D.R., H.M. van Eijk, and N.E. Deutz, Simple and sensitive multi-sugar-probe gut permeability test by high-performance liquid chromatography with fluorescence labelling. J Chromatogr A, 1996. 730(1-2): p. 99-105.

30. van Wijck, K., et al., Novel analytical approach to a multi-sugar whole gut permeability assay. J Chromatogr B Analyt Technol Biomed Life Sci, 2011. 879(26): p. 2794-801.

31. Fink, M.P., Clinical tests of gastrointestinal permeability that rely on the urinary recovery of enterally administered probes can yield invalid results in critically ill patients. Intensive Care Med, 2002. 28(2): p. 103-4.

32. Farhadi, A., et al., Susceptibility to gut leakiness: a possible mechanism for endotoxaemia in nonalcoholic steatohepatitis. Liver Int, 2008. 28(7): p. 1026-33.

33. Farhadi, A., et al., Gas chromatographic method for detection of urinary sucralose: application to the assessment of intestinal permeability. J Chromatogr B Analyt Technol Biomed Life Sci, 2003. 784(1): p. 145-54.

34. Charlson, M.E., et al., A new method of classifying prognostic comorbidity in longitudinal studies: development and validation. J Chronic Dis, 1987. 40(5): p. 373-83.

35. Pijls, K.E., et al., Large intestine permeability is increased in patients with compensated liver cirrhosis. Am J Physiol Gastrointest Liver Physiol, 2014. 306(2): p. G147-53.

36. Ohri, S.K., et al., The effect of intestinal hypoperfusion on intestinal absorption and permeability during cardiopulmonary bypass. Gastroenterology, 1994. 106(2): p. 318-23.

37. Riddington, D.W., et al., Intestinal permeability, gastric intramucosal pH, and systemic endotoxemia in patients undergoing cardiopulmonary bypass. JAMA, 1996. 275(13): p. 1007-12.

38. Braun, J.P., et al., Splanchnic oxygen transport, hepatic function and gastrointestinal barrier after normothermic cardiopulmonary bypass. Acta Anaesthesiol Scand, 2004. 48(6): p. 697-703.

39. Doig, C.J., et al., Increased intestinal permeability is associated with the development of multiple organ dysfunction syndrome in critically ill ICU patients. Am J Respir Crit Care Med, 1998. 158(2): p. 444-51.

40. Johnston, J.D., et al., Gastrointestinal permeability and absorptive capacity in sepsis. Crit Care Med, 1996. 24(7): p. 1144-9.

41. de Oliveira, E.P., R.C. Burini, and A. Jeukendrup, Gastrointestinal complaints during exercise: prevalence, etiology, and nutritional recommendations. Sports Med, 2014. 44 Suppl 1: p. S79-85.

42. van Wijck, K., et al., Exercise-induced splanchnic hypoperfusion results in gut dysfunction in healthy men. PLoS One, 2011. 6(7): p. e22366.

43. Oktedalen, O., et al., Changes in the gastrointestinal mucosa after long-distance running. Scand J Gastroenterol, 1992. 27(4): p. 270-4.

44. van Nieuwenhoven, M.A., F. Brouns, and R.J. Brummer, Gastrointestinal profile of symptomatic athletes at rest and during physical exercise. Eur J Appl Physiol, 2004. 91(4): p. 429-34.

45. Hundscheid, I.H., et al., The Human Colon Is More Resistant to Ischemia-reperfusion-induced Tissue Damage Than the Small Intestine: An Observational Study. Ann Surg, 2015. 262(2): p. 304-11.

46. Takala, J., Determinants of splanchnic blood flow. Br J Anaesth, 1996. 77(1): p. 50-8.

47. Grootjans, J., et al., Human intestinal ischemia-reperfusion-induced inflammation characterized: experiences from a new translational model. Am J Pathol, 2010. 176(5): p. 2283-91.

48. Nayci, A., et al., Bronchoscopy is associated with decreased mesenteric arterial flow. Crit Care Med, 2008. 36(9): p. 2517-22.

49. Parks, D.A. and E.D. Jacobson, Physiology of the splanchnic circulation. Arch Intern Med, 1985. 145(7): p. 1278-81.

50. Wang, X., et al., Cardiac autonomic function in patients with acute exacerbation of chronic obstructive pulmonary disease with and without ventricular tachycardia. BMC Pulm Med, 2016. 16(1): p. 124.

51. Andreas, S., et al., Increased sympathetic nerve activity in COPD is associated with morbidity and mortality. Lung, 2014. 192(2): p. 235-41. 
52. Bathoorn, E., et al., Airways inflammation and treatment during acute exacerbations of COPD. Int J Chron Obstruct Pulmon Dis, 2008. 3(2): p. 217-29.

53. Gan, W.Q., et al., Association between chronic obstructive pulmonary disease and systemic inflammation: a systematic review and a meta-analysis. Thorax, 2004. 59(7): p. 574-80.

54. Reijnders, D., et al., Effects of Gut Microbiota Manipulation by Antibiotics on Host Metabolism in Obese Humans: A Randomized Double-Blind Placebo-Controlled Trial. Cell Metab, 2016. 24(2): p. 341.

55. Barthelemy, J.C., et al., Accuracy of pulse oximetry during moderate exercise: a comparative study. Scand J Clin Lab Invest, 1990. 50(5): p. 533-9. 



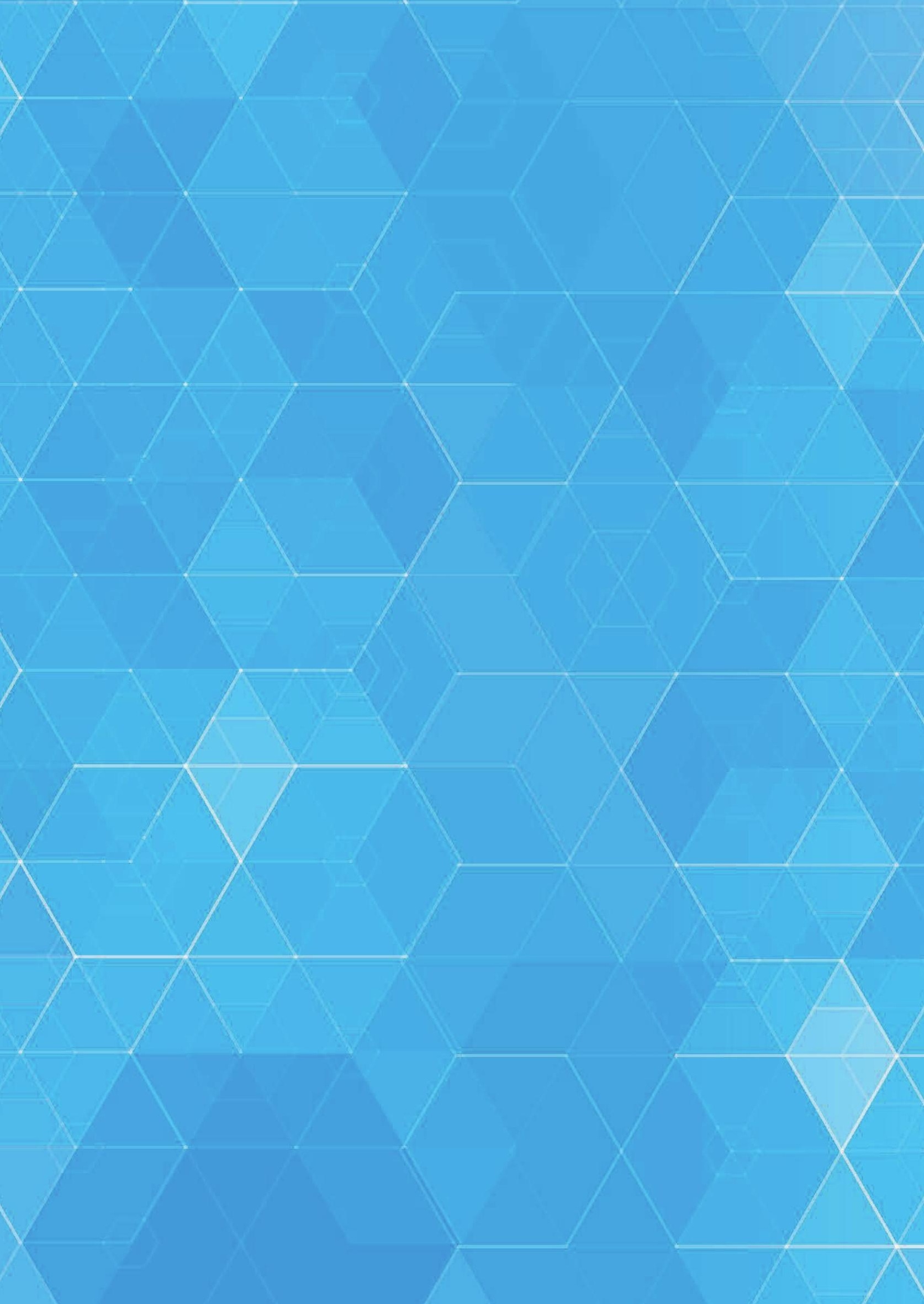




\section{Chapter 9}

Gut integrity in relation to exacerbations in patients with chronic obstructive pulmonary disease

Roy T. M. Sprooten, Emiel F.M. Wouters, Kaatje Lenaerts, Niki L. Reynaert, Miriam T.J. Groenen, Frits M.E. Franssen 


\section{Abstract}

\section{Background}

Involvement of the disturbed gastro-intestinal (GI) integrity has been previously observed in patients with chronic obstructive pulmonary disease (COPD), but its relation with systemic inflammation and/or increased risk of exacerbations is unknown. This study aimed $(\mathrm{I})$ to compare plasma concentrations of Intestinal Fatty Acid-Binding Protein (I-FABP), reflecting GI integrity between COPD patients and non-COPD controls, and (II) to investigate whether I-FABP is increased in patients with frequent exacerbations compared to those without, (III) to assess whether higher I-FABP levels predict exacerbations during follow-up, and (IV) to study the association between the $\mathrm{Gl}$ integrity and systemic inflammation in COPD patients and smoking and non-smoking controls.

\section{Methods}

Plasma concentrations of I-FABP were determined in participants of the ICE-AGE study, an observational study with a longitudinal follow-up of 2 years.

\section{Results}

Two-hundred-and-five patients with COPD (58\% males, age: $62.2 \pm 7.0 \mathrm{y}, \mathrm{FEV}_{1}$ : $49.4 \pm 15.6 \%$ ) and 200 non-COPD controls (45\% males, age: $60.8 \pm 6.5$ y) were included. IFABP levels were significant higher in COPD compared to controls (Median(IQR) 1113.6(828.5-1735.9) pg/ml vs 950.2(707.2-1482.2) pg/ml; $\mathrm{p}=0.005)$. I-FABP levels were not related to previous exacerbation history. COPD patients with frequent exacerbations or hospital admissions for exacerbation of COPD during follow-up had comparable I-FABP levels to those without these events. I-FABP was positively related to plasma IL-8 levels in COPD (Spearman $r=0.2082 ; p<0.005$ ).

\section{Conclusion}

I-FABP is increased in COPD compared to non-COPD, reflecting GI disintegrity. However, associations with risk of exacerbations were not observed. Furthermore, a limited association with systemic inflammation was found. 


\section{Introduction}

Chronic obstructive pulmonary disease (COPD) is a clinical syndrome characterized by a combination of chronic respiratory symptoms, structural pulmonary abnormalities and lung function impairment. ${ }^{1}$ Exacerbations of COPD (ECOPD) play a pivotal role in the morbidity and progression of the disease. ${ }^{2}$ Bacterial and viral infections, hypoxemia, and local and systemic inflammation are common during ECOPD., ${ }^{3,4}$ Next to the pulmonary abnormalities, patients with COPD frequently have extra-pulmonary features and coexisting chronic conditions ${ }^{5}$ which contribute to its clinical heterogeneity. ${ }^{6}$ Involvement of the gastro-intestinal (GI) tract as part of the multimorbid pathology in COPD was previously suggested. ${ }^{7}$ Indeed, the majority of COPD patients report $\mathrm{Gl}$ symptoms. ${ }^{8}$ In addition, (patho)physiological interactions between the $\mathrm{GI}$ tract and the respiratory tract are beginning to be understood. ${ }^{9}$ Changes in gut microbiota may be linked to altered systemic and pulmonary immunity, especially when the gut barrier function is reduced ${ }^{10}$ and bacterial translocation across the intestinal wall may occur. In COPD, increased GI permeability and enterocyte damage compared to controls were observed following performance of activities of daily living in a small study. ${ }^{11}$ Vice versa, triggers associated with COPD and its acute exacerbations, including smoking, hypoxemia and infections may cause GI disruption and microbial dysbiosis. ${ }^{9}$ Increased GI permeability was indeed also reported in COPD patients hospitalized for acute exacerbations with hypoxemic respiratory failure ${ }^{12}$, compared to stable disease. It is currently unknown whether GI permeability differs between COPD patients with frequent versus infrequent exacerbations, those with less severe versus more severe disease, and whether it is associated with systemic inflammation. The current study aimed to increase our understanding of the association between GI integrity, ECOPD and systemic inflammation. Specifically, the aims of the present study were (I) to assess the GI integrity in a large sample of patients with COPD and non-COPD controls, (II) to study difference in GI integrity between patients with a history of frequent exacerbations compared to those without, (III) to assess whether GI integrity status predicts exacerbations during long-term follow-up and (IV) to furthermore study the association between the GI integrity and systemic inflammation in COPD patients and smoking and non-smoking controls.

\section{Materials and methods}

The present analyses are based on the "Individualized COPD Evaluation in relation to Ageing" (ICE-Age) study. ${ }^{13}$ This was a single-center, observational, longitudinal study 
with a follow-up period of 2 years. In total 405 subjects were recruited in the ICE AGE study: 205 clinically stable COPD patients, and 200 non-COPD smoking (>10 pack years) and non-smoking ( $\leq 10$ pack years) controls. Inclusion criteria for COPD patients were: diagnosis of COPD according to the GOLD guidelines $\left(\mathrm{FEV}_{1}<80 \%\right.$ predicted and $\mathrm{FEV}_{1} / \mathrm{FVC}<70 \%{ }^{14}$, age between $45-74$ years and absence of respiratory tract infection or ECOPD within 4 weeks before inclusion. Male and female controls were excluded if one of the following diagnoses was present: COPD, heart failure or other cardiovascular pathology, renal failure, untreated diabetes mellitus type II, severe and untreated osteoporosis. Exclusion criteria for both groups were: any kind of carcinogenic pathology $<5 y$ before the study; chronic use of corticosteroids $>10 \mathrm{mg} /$ day; investigator uncertainty about willingness or ability of the subject to comply with the protocol requirements and participation in a study involving investigational or marketed products concomitantly or $<4$ weeks prior to study entry. ${ }^{13}$ The study was approved by the ethical committee of the Maastricht University Medical Centre (MUMC, METC 10-3-033, METC 2019-0992), and conducted according to the Declaration of Helsinki $\left(59^{\text {nd }}\right.$ WMA General Assembly, Seoul, October 2008) and Good Clinical Practice guidelines. Before enrollment all participants gave written informed consent.

\section{Data collection}

Demographic, clinical and laboratory parameters were collected from the electronic records of the patients or assessed at entry in the study. The following variables were scored: medical history and relevant comorbidities, quantified using the Charlson comorbidity index $(\mathrm{CCl})^{15}$, number of pack years smoked, smoking status, medication as well as use of home oxygen, history of moderate and severe exacerbations in the last 12 months. ${ }^{2}$ At study entry, post-bronchodilator lung forced expiratory volume in the first second $\left(\mathrm{FEV}_{1}\right)$, forced vital capacity (FVC) and $\mathrm{FEV}_{1} / \mathrm{FVC}$ ratio, as well as residual volume (RV), total lung capacity (TLC), diffusion capacity (DLCO) were measured (Masterlab, Jaeger). Arterial blood gas analysis was performed in the COPD group. Blood samples for assessment of metabolic and inflammatory parameters were collected at the start of the study under fasting conditions. During follow-up, moderate and severe ECOPD ${ }^{2}$ and all-cause mortality were prospectively recorded by threemonthly phone interviews. To non-invasively evaluate gut integrity, plasma intestinalfatty-acid binding protein (I-FABP) levels were measured by an in-house developed enzyme-linked immunosorbent assay (ELISA). ${ }^{16}$ I-FABP is a small, $15-k D$ cytosolic protein that is present especially in mature enterocytes of the small intestine. ${ }^{16-18}$ Upon enterocyte injury, the protein rapidly diffuses through the interstitial space into the circulation, enabling its detection in plasma samples. ${ }^{19,20}$ Therefore, I-FABP has 
emerged as a non-invasive marker for detection of enterocyte damage and evaluation of gut wall integrity. ${ }^{21-23}$ The detection window of the I-FABP assay is 12.5 to $800 \mathrm{pg} / \mathrm{mL}$. The intra-assay and inter-assay coefficient of variation of this assay are $4.1 \%$ and $6.2 \%$, respectively. Three different FABPs are known: I-FABP, liver FABP and ileal lipid binding protein. The assay is specific for the detection of the intestinal FABP isoform. ${ }^{17}$ Circulating leucocytes, C-reactive protein (CRP), interleukin-6 (IL-6) and interleukin-8 (IL-8), as well as fibrinogen were measured at baseline. Leukocytes were analyzed by Sysmex XN9000 and CRP was measured in EDTA plasma using an auto-analyzer (ABX Pentra 400, HORIBA ABXS.A.S, France). Plasma IL-6 and IL-8were measured using validated immunoassays (R\&D Systems, Inc., Minneapolis, USA). Fibrinogen was assessed using citrate plasma in a coagulation analyzer (Sysmex CA-7000; DadeBehring, Germany).

\section{Statistical analysis}

SPSS version 25 software was used for all statistical analyses. First, descriptive analysis was performed. Distribution of the variables was assessed by Kolmogorov-Smirnov test and normally distributed data are presented as means and standard deviations, whereas non-parametric data are presented as medians and first and third quartiles (25-75\% percentiles). Categorical data are presented as percentages ( $n(\%)$ ). Patients were categorized as frequent or infrequent exacerbators, spirometric stages of disease and symptoms according to GOLD. ${ }^{2,14}$ To compare the characteristics of COPD patients and non-COPD control groups, the Mann-Whitney $U$ test or T-test for continuous variables and the Chi square test for categorical data were used. The COPD patients were divided according exacerbation frequency during follow-up (0-1 ECOPD vs. >2 ECOPD; and no H-ECOPD vs. >1 H-ECOPD) and to analyze the differences between these groups we used the ANOVA. Furthermore, Kaplan Meier analysis was performed into time to first hospitalized ECOPD related to I-FABP levels categorized by three groups: $<33 \%$ tertile, $33-66 \%$ tertile, $>66 \%$ tertile. The Pearson or Spearman correlation test was performed to investigate correlations between I-FABP and the occurrence of ECOPD and other relevant variables especially inflammatory mediators (IL-8, IL-6, Fibrinogen, CRP). A p-value $<0.05$ was considered statistically significant.

\section{Results}

\section{Characteristics study population}

Baseline characteristics of the COPD patients and non-COPD controls included in ICEAge have been published before ${ }^{6}$ and are briefly summarized in Table 9.1. On average, 
ICE-Age included elderly, overweight, (former) smoking COPD patients with moderate to very severe airflow limitation representing both genders. The majority of patients had at least 1 ECOPD in the previous year and were multi-morbid. Controls had a comparable age and $\mathrm{BMI}$, were more frequently females, had less pack years and normal lung function.

Table 9.1 Baseline characteristics.

\begin{tabular}{|c|c|c|c|}
\hline Variable & $\begin{array}{c}\text { COPD } \\
(n=205)\end{array}$ & $\begin{array}{l}\text { Controls } \\
(n=200)\end{array}$ & p-value \\
\hline Male gender & $119(58.0)$ & $90(45.0)$ & 0.009 \\
\hline Age, y & $62.3 \pm 7.0$ & $60.8 \pm 6.5$ & 0.028 \\
\hline $\mathrm{BMI}, \mathrm{kg} / \mathrm{m}^{2}$ & $27.0 \pm 5.4$ & $26.9 \pm 3.4$ & 0.819 \\
\hline \multicolumn{4}{|l|}{ Smoking status } \\
\hline Pack years* $(n=204 / 195)$ & $48.6 \pm 28.9$ & $12.3 \pm 14.9$ & 0.0001 \\
\hline Former & $174(84.1)$ & $115(57.5)$ & 0.0001 \\
\hline Current & $28(13.7)$ & $26(13.0)$ & \\
\hline Never & $3(1.4)$ & $59(29.5)$ & \\
\hline MMRC-D scale & $3.0 \pm 1.0$ & NA & NA \\
\hline \multicolumn{4}{|l|}{ Spirometric GOLD stage } \\
\hline 1 & $1(0.5)$ & NA & NA \\
\hline II & $100(48.3)$ & NA & NA \\
\hline III & $84(41.0)$ & NA & NA \\
\hline IV & $20(9.8)$ & NA & NA \\
\hline \multicolumn{4}{|l|}{ Lung function } \\
\hline $\mathrm{FEV}_{1}$, post $\mathrm{L}$ & $1.39 \pm 0.51$ & $3.32 \pm 0.73$ & 0.0001 \\
\hline $\mathrm{FEV}_{1}$, post \%pred & $49.6 \pm 15.6$ & $119.1 \pm 15.0$ & 0.0001 \\
\hline $\mathrm{FEV}_{1} / \mathrm{FVC}$ & $41.1 \pm 11.4$ & $78.5 \pm 4.6$ & 0.0001 \\
\hline RV, \%pred $(n=202 / 200)$ & $158.7 \pm 45.1$ & $94.9 \pm 17.7$ & 0.0001 \\
\hline TLCO, \%pred* ( $n=199 / 199)$ & $54.6 \pm 18.3$ & $93.3 \pm 13.4$ & 0.0001 \\
\hline \multicolumn{4}{|l|}{ Blood gasses $(n=121)$} \\
\hline $\mathrm{PaCO}_{2}, \mathrm{kPa}$ & $5.1 \pm 0.8$ & NA & NA \\
\hline $\mathrm{PaO}_{2}, \mathrm{kPa}(\mathrm{n}=121)$ & $9.9 \pm 1.5$ & NA & NA \\
\hline $\mathrm{SpO}_{2}, \% *$ & $94.0(93.0-96.0)^{*}$ & $98.0(97.0-98.0)^{*}$ & 0.0001 \\
\hline LTOT & $32(15.6)$ & NA & NA \\
\hline \multicolumn{4}{|l|}{ Exacerbation history } \\
\hline No ECOPD previous year & $45(22.1)$ & NA & NA \\
\hline ECOPD 0-1 previous year & $108(52.7)$ & NA & NA \\
\hline ECOPD $>1$ previous year & $97(47.3)$ & NA & NA \\
\hline H-ECOPD previous year $(n=204 / 194)$ & $63(30.7)$ & NA & NA \\
\hline \multicolumn{4}{|l|}{ Co-morbidities ( $n=201 / 200)$} \\
\hline $\mathrm{CCl} 0$ points & $91(45.3)$ & $139(69.5)$ & 0.0001 \\
\hline $\mathrm{CCl} 1$ point & $73(36.3)$ & $50(25.0)$ & \\
\hline $\mathrm{CCl} \geq 2$ points & $37(18.4)$ & $11(5.5)$ & \\
\hline
\end{tabular}

Categorical data are presented as $\mathrm{n}(\%)$, non-parametric data* as median (IQR) and parametric data as mean \pm (SD). Chi- $X^{2}$, Kruskal Wallis test/ Mann Whitney test and T-test were used for statistical analysis respectively. Abbrevations: BMI: body mass index; LTOT: Long-term Oxygen therapy; ECOPD: exacerbation of COPD; HECOPD: hospitalised exacerbation of COPD; CCI: Charlson Comorbidity Index. 
During the 2 years follow-up 186 (94.6\%) COPD patients had an ECOPD, of whom 118 (63.4\%) patients had 2 or more ECOPD. 54 (32.1\%) patients underwent a hospital admission for ECOPD. During the follow-up period 12 patients died and 3 patients withdrew from the study.

Baseline plasma levels of the inflammatory parameters CRP, leucocytes, IL-6, IL-8 and fibrinogen were significantly increased in the COPD group compared to the control group (Table 9.2).

Table 9.2 Baseline inflammatory parameters.

\begin{tabular}{|c|c|c|c|}
\hline Parameter & $\begin{array}{c}\text { COPD } \\
(n=205)\end{array}$ & $\begin{array}{l}\text { Controls } \\
(n=200)\end{array}$ & p-value \\
\hline CRP, mg/l ( $n=204 / 195)$ & $2.9(0.9-7.1)$ & $0.8(0.35-1.6)$ & 0.0001 \\
\hline Leucocytes, $10^{9} / \mathrm{L}$ ( $=186 / 183$ & $7.3(6.0-8.1)$ & $5.4(4.5-6.6)$ & 0.0001 \\
\hline IL-6, pg/ml (n=199/191) & $5.8(3.2-11.7)$ & $4.6(1.9-13.4)$ & 0.0300 \\
\hline IL-8, pg/ml (n=199/191) & $7.8(3.7-12.7)$ & $4.6(0.36-8.7)$ & 0.0001 \\
\hline Fibrinogen, $g / l(n=194 / 188)$ & $3.1(2.7-3.7)$ & $2.6(2.4-3.0)$ & 0.0001 \\
\hline
\end{tabular}

Non-parametric data as median (IQR), Kruskal Wallis test was used for statistical analysis. Abbrevations: IFABP: Intestinal fatty acid binding protein; CRP: C-reactive protien; IL: interleukin.

\section{I-FABP and COPD}

Plasma I-FABP levels at baseline were significantly higher in COPD subjects compared to controls, respectively $1113.6 \mathrm{pg} / \mathrm{ml}$ (828.5-1735.9) vs. $950.2 \mathrm{pg} / \mathrm{ml}$ (707.2-1482.2) $(p=0.005)(n=202 / 199)$ (Figure 9.1A). No significant differences in I-FABP levels were found between different spirometric GOLD stages or quadrants (ANOVA $F=0.542$ p 0.719; F=0.226; p 0.878 respectively) (Figure 9.2A and 9.2B).

There was no difference in baseline I-FABP levels between COPD patients with 0-1 versus 2 or more moderate exacerbations during follow up $(p=0.911)$, or between those with 0 versus 1 of more severe exacerbation during follow up $(p=0.580)$.

As illustrated in Figure 9.3, the time to first admission of ECOPD did not differ between tertiles of I-FABP levels.

I-FABP levels did not differ significantly between the never smokers vs current and former smokers in the control group $(1113.4 \pm 603.7 \mathrm{pg} / \mathrm{ml} ; 1164.9 \pm 6862 \mathrm{pg} / \mathrm{ml}$; $\mathrm{p}=0.619$ ), and within the COPD group, no difference was found between former smokers versus current smokers (1380.9 $\pm 866.2 \mathrm{pg} / \mathrm{ml} ; 1429.1 \pm 992.4 \mathrm{pg} / \mathrm{ml} ; \mathrm{p}=0.792)$. 


\section{Systemic inflammation and I-FABP}

In COPD, I-FABP levels were significantly related to IL-8 (Spearman's rho( $r)=0.206$; $p<0.005)$ whereas in the controls no significant correlation was found $(r=0.63, p=0.194)$ (Figure 9.1B and 9.4). No other relationships between I-FABP and markers of systemic inflammation were found.

Figure 9.1 A: Boxplot: Plasma I-FABP levels in controls versus COPD patients ( $n=202 / 199)$. Respectively 950.2 (707.2-1482.2) pg/ml vs 1113.6 (828.5-1735.9) pg/ml ( $p=0.005)$;

B: Boxplot: Plasma IL-8 levels in controls versus COPD patients ( $n=199 / 191)$. Respectively 4.6 $(0.36-8.7) \mathrm{pg} / \mathrm{ml}$ vs $7.8(3.7-12.7) \mathrm{pg} / \mathrm{ml}(\mathrm{p}=0.0001)$.

A

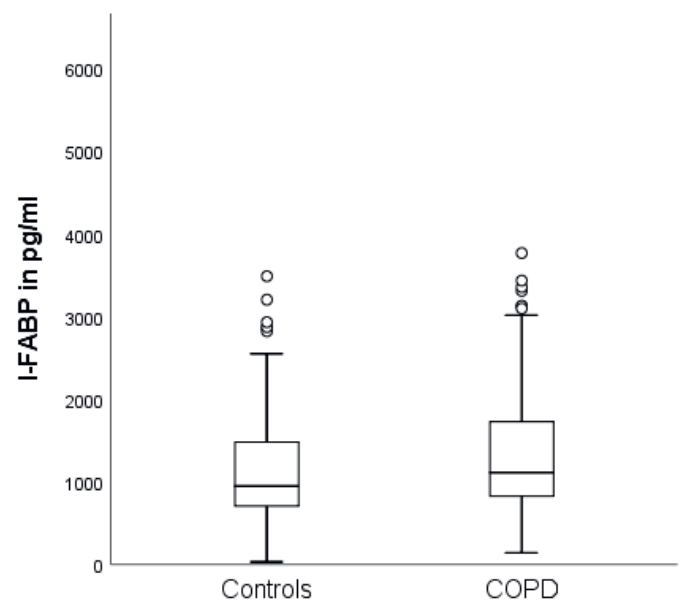

B

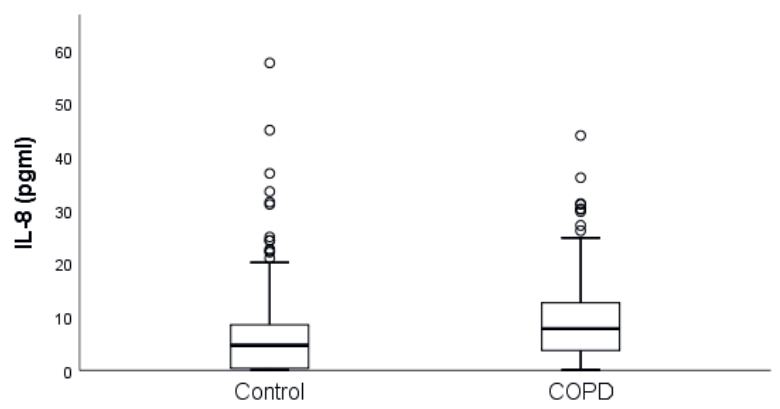

Abbrevations: I-FABP: Intestinal tatty acid binding protein; IL: interleukin. 
Figure 9.2 Boxplots: Plasma I-FABP levels in COPD patients classified according GOLD Stage A:(Spirometric I-IV) and $B:(A-D)$.

A

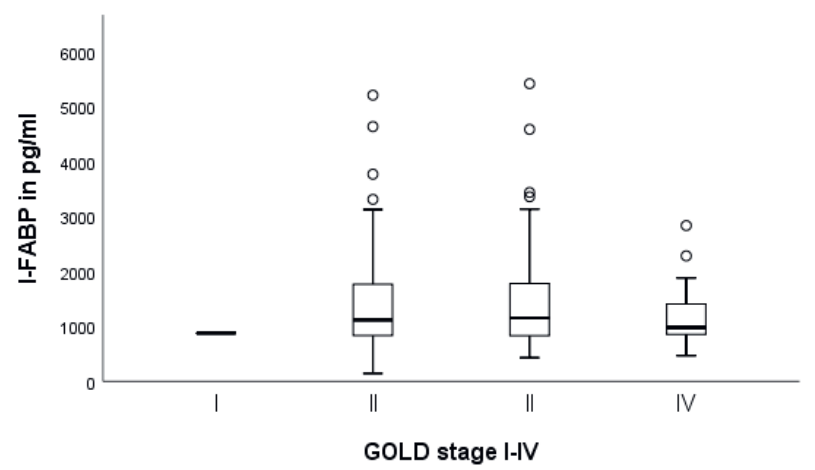

B

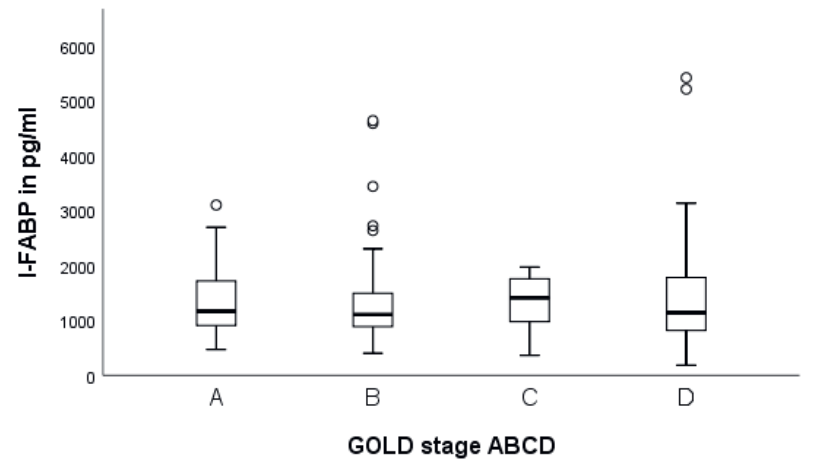

Abbrevations: I-FABP: Intestinal fatty acid binding protein.

Figure 9.3 Kaplan Meier curve depicting time to first hospitalized ECOPD (H-ECOPD) during two years follow-up. The I-FABP levels are categorized in: IQR<33\%; IQR 33-66\%; IQR >66\%. (Log rank=0.594; Breslow =0.590, not significant).

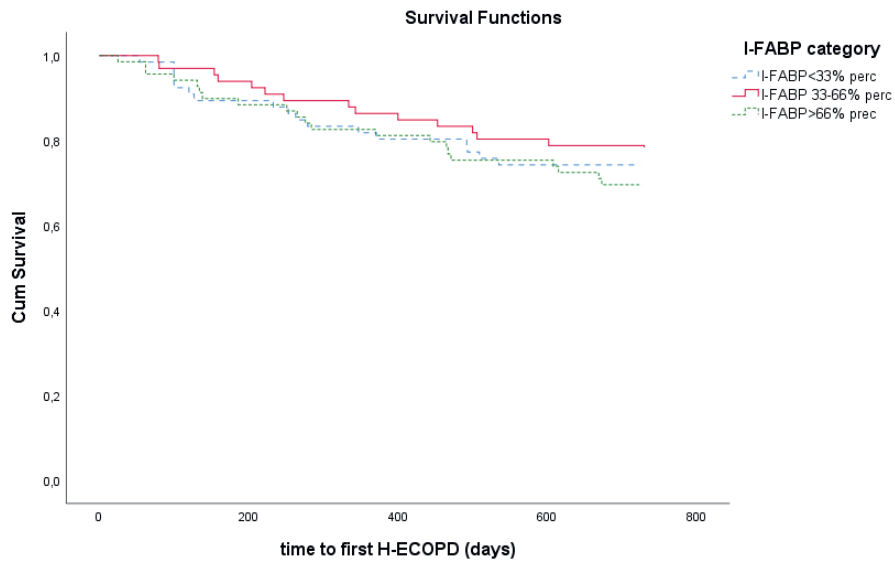


Figure 9.4 Correlation I-FABP levels and IL-8 levels. A: COPD group: Spearman's rho (r)=0.206 with $\mathrm{p}=0.002$; : Control group: $r=0.63 ; p=0.194$, not significant.

COPD group

A

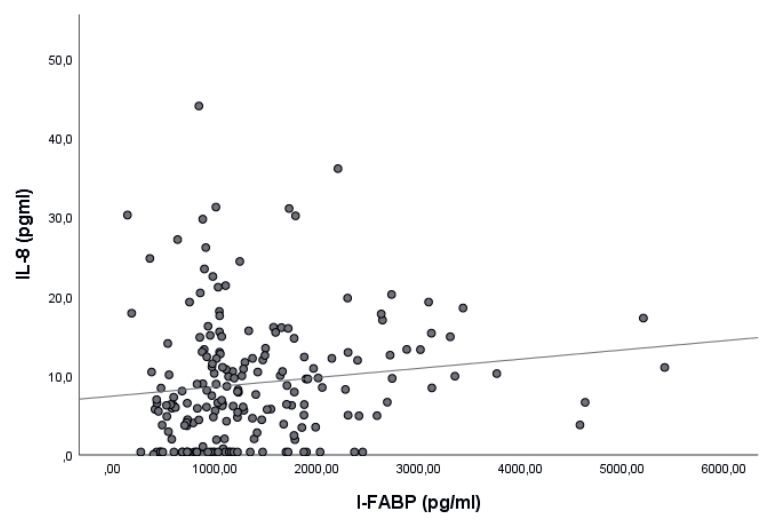

Control group

B

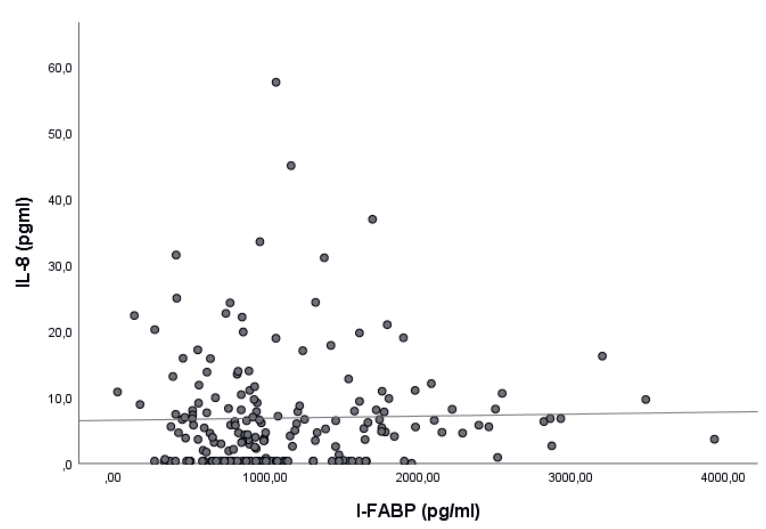

Abbrevations: I-FABP: Intestinal fatty acid binding protein; IL-8: Interleukin-8.

\section{Discussion}

The present study was the first to investigate the associations between GI integrity, exacerbations and systemic inflammation in a large sample of COPD patients and nonCOPD controls. It shows that GI integrity is decreased in stable-state COPD compared to non-COPD. GI integrity in COPD was not related to the severity of airflow limitation or 2020 GOLD classification. No difference in GI integrity was observed between COPD 
patients with or without frequent exacerbations or hospitalizations. Also, baseline $\mathrm{Gl}$ integrity was not predictive of exacerbations during follow-up. Finally, the study showed that decreased GI integrity is associated with increased levels of IL-8, but not with other markers of systemic inflammation. Hence, although gut-lung interactions are frequently suggested in COPD and recently reported as disturbed permeability during exacerbation of COPD, this study did not confirm an association between GI integrity and exacerbation risk in patients with moderate to severe COPD.

Increased I-FABP levels have been reported in several acute conditions including septic, haemorrhagic or cardiogenic shock ${ }^{24}$, cardiac surgery complicated with multiple organ dysfunction syndrome or mesenteric ischemia. ${ }^{25,26}$ Elevated serum I-FABP concentrations were also found in patients with acute ischemic stroke and provide evidence for the involvement of a disrupted intestinal integrity in these patients. ${ }^{27}$ In patients with pneumonia treated in the intensive care unit high I-FABP levels measured in urine were associated with poor outcome. ${ }^{28}$ Recently, in patients not critically ill but in the more stable course of sickle cell disease, higher levels of serum I-FABP compared to controls were reported. ${ }^{29}$

The role of the gut is increasingly recognized in chronic diseases, including chronic heart failure (CHF), another multicomponent, chronic organ disease characterized by a combination of increased sympathetic tone, anabolic/catabolic disbalance and chronic inflammation. ${ }^{57}$ Even in stable, compensated CHF patients an increased intestinal paracellular permeability is reported. ${ }^{58}$ In patients with acute heart failure or cardiogenic shock, increased I-FABP levels were also found and were associated with high mortality, suggesting that inadequate perfusion of the small intestine might play a pivotal role in the worse outcome. ${ }^{30}$ In stable COPD patients, Rutten et al reported significantly elevated plasma I-FABP levels measured after performing daily life activities, indicating functional alterations in the $\mathrm{Gl}$ tract. ${ }^{11}$ The redistribution of blood flow from the splanchnic region to the exercising muscles could be a contributing factor in this temporary increase in I-FABP levels. ${ }^{31}$

Alterations in the intestinal microbiome or dysbiosis in relation to gut compromise need further exploration as dysbiosis contributes to a plethora of important host functions. $^{32}$ Gut dysbiosis is poorly analysed in relation to COPD $^{33}$, but is associated with increased intestinal permeability. ${ }^{34}$ High levels of Proteobacteria are reported as a key feature of dysbiosis in many chronic inflammatory and metabolic conditions. ${ }^{35,36} \mathrm{Gram}$ negative bacteria produce different endotoxins with major variation in the immunogenetic lipid A portion that binds to toll like receptor-4 (TLR-4). ${ }^{37}$ Future studies 
need to explore the gut integrity loss in relation to Proteobacteria dysbiosis in stable COPD patients and during acute events.

A common hallmark of chronic inflammation in the respiratory and gastro-intestinal epithelium is a structural and functional disruption of apical junctions. ${ }^{38}$ Many mediators of innate and adaptive immunity may be increased upon chronic cigarette smoke exposure or indirectly by their impact on the microbiome. ${ }^{39}$ Among cytokines, especially T-helper (Th) 2 and 17 cytokines have been proposed as key disruptive factors for epithelial integrity. ${ }^{40,41}$ Further studies will be needed to unravel this gutairway barrier link as part of a generalised epithelial barrier dysfunction at least in subtypes of COPD. Also, the role of microvascular dysfunction ${ }^{42}$ in gut dysfunction in COPD is currently unexplored.

Intriguing is our finding of an association between gut integrity loss and systemic inflammation. Previous studies already postulated that translocation of luminal gut antigens to the systemic circulation may be a pathogenic factor in different chronic conditions. $^{43-45}$ Elevated levels of endotoxin or bacterial lipopolysaccharide (LPS) from the outer membrane of gram-negative bacteria are associated with a threefold increased risk of developing atherosclerosis. ${ }^{46}$ Endotoxin binds TLR-4 receptors present on the intestinal, immune and endothelial cells and forms a TLR-4 receptor cluster with the Cluster of Differentiation-14 (CD-14). ${ }^{47}$ It has been reported that systemic inflammation is only present in one third of COPD patients and is not a constant feature over time. ${ }^{48}$ Further studies are needed to unravel this link between systemic inflammation, endotoxin and microbial dysbiosis.

\section{Strengths and limitations}

Strengths of this study include the comprehensive patient assessment, sample size, inclusion of a control group and longitudinal follow-up. Furthermore, to our knowledge this is the first study of COPD patients at risk for frequent exacerbations that investigated the levels of I -FABP levels in relation to systemic inflammation.

Our results should be interpreted in light of some limitations. First, the presented study was performed in a single, tertiary care center, including patients with high burden of disease, which limits the generalizability of our results to other care settings. Moreover, we exclusively used I-FABP as a marker for GI integrity. Other tests, such as the intestinal permeability test using the multi-sugar test ${ }^{49}$ were not performed. Another limitation is the omission to assess the cardiac status thoroughly as contributing factor 
to impaired the tissue oxygen delivery. However, all patients were clinically stable during the baseline study visit.

\section{Conclusions}

In conclusion, this study reported loss of GI integrity in stable COPD patients compared to controls, with no association with exacerbations and only limited association with systemic inflammation. Further studies are warranted before the potential presence of a clinically relevant gut-lung axis can be confirmed. 


\section{References}

1. Celli BR, Wedzicha JA. Update on Clinical Aspects of Chronic Obstructive Pulmonary Disease. N Engl J Med. 2019;381(13):1257-66.

2. WHO. Global strategy for the diagnosis, management, and prevention of chronic obstructive pulmonary disease, 2020 report. 2019.

3. Viniol C, Vogelmeier CF. Exacerbations of COPD. European respiratory review : an official journal of the European Respiratory Society. 2018;27(147).

4. Thomsen M, Ingebrigtsen TS, Marott JL, Dahl M, Lange P, Vestbo J, et al. Inflammatory biomarkers and exacerbations in chronic obstructive pulmonary disease. JAMA : the journal of the American Medical Association. 2013;309(22):2353-61.

5. Vanfleteren L, Spruit MA, Wouters EFM, Franssen FME. Management of chronic obstructive pulmonary disease beyond the lungs. Lancet Respir Med. 2016;4(11):911-24.

6. Triest FJJ, Franssen FME, Reynaert N, Gaffron S, Spruit MA, Janssen DJA, et al. Disease-Specific Comorbidity Clusters in COPD and Accelerated Aging. J Clin Med. 2019;8(4).

7. Keely S, Talley NJ, Hansbro PM. Pulmonary-intestinal cross-talk in mucosal inflammatory disease. Mucosal Immunol. 2012;5(1):7-18.

8. Rutten EP, Spruit MA, Franssen FM, Buurman WA, Wouters EF, Lenaerts K. GI symptoms in patients with COPD. Chest. 2014;145(6):1437-8.

9. Budden KF, Gellatly SL, Wood DL, Cooper MA, Morrison M, Hugenholtz P, et al. Emerging pathogenic links between microbiota and the gut-lung axis. Nat Rev Microbiol. 2017;15(1):55-63.

10. Turner JR. Intestinal mucosal barrier function in health and disease. Nat Rev Immunol. 2009;9(11):799809.

11. Rutten EPA, Lenaerts K, Buurman WA, Wouters EFM. Disturbed intestinal integrity in patients with COPD: effects of activities of daily living. Chest. 2014;145(2):245-52.

12. Sprooten RTM, Lenaerts K, Braeken DCW, Grimbergen I, Rutten EP, Wouters EFM, et al. Increased Small Intestinal Permeability during Severe Acute Exacerbations of COPD. Respiration. 2018;95(5):334-42.

13. Rutten EP, Gopal P, Wouters EF, Franssen FM, Hageman GJ, Vanfleteren LE, et al. Various Mechanistic Pathways Representing the Aging Process Are Altered in COPD. Chest. 2016;149(1):53-61.

14. Vogelmeier CF, Criner GJ, Martinez FJ, Anzueto A, Barnes PJ, Bourbeau J, et al. Global Strategy for the Diagnosis, Management, and Prevention of Chronic Obstructive Lung Disease 2017 Report. GOLD Executive Summary. Am J Respir Crit Care Med. 2017;195(5):557-82.

15. Charlson ME, Pompei P, Ales KL, MacKenzie CR. A new method of classifying prognostic comorbidity in longitudinal studies: development and validation. J Chronic Dis. 1987;40(5):373-83.

16. Van Wijck K, Lenaerts K, Van Bijnen AA, Boonen B, Van Loon L, Dejong CH, et al. Aggravation of exercise-induced intestinal injury by Ibuprofen in athletes. Med Sci Sports Exerc. 2012;44(12):2257-62.

17. Pelsers MM, Namiot Z, Kisielewski W, Namiot A, Januszkiewicz M, Hermens WT, et al. Intestinal-type and liver-type fatty acid-binding protein in the intestine. Tissue distribution and clinical utility. Clin Biochem. 2003;36(7):529-35.

18. Agellon LB, Toth MJ, Thomson AB. Intracellular lipid binding proteins of the small intestine. Mol Cell Biochem. 2002;239(1-2):79-82.

19. Lieberman JM, Sacchettini J, Marks C, Marks WH. Human intestinal fatty acid binding protein: report of an assay with studies in normal volunteers and intestinal ischemia. Surgery. 1997;121(3):335-42.

20. Pelsers MM, Hermens WT, Glatz JF. Fatty acid-binding proteins as plasma markers of tissue injury. Clin Chim Acta. 2005;352(1-2):15-35.

21. Adriaanse MP, Tack GJ, Passos VL, Damoiseaux JG, Schreurs MW, van Wijck K, et al. Serum I-FABP as marker for enterocyte damage in coeliac disease and its relation to villous atrophy and circulating autoantibodies. Aliment Pharmacol Ther. 2013;37(4):482-90.

22. Vreugdenhil AC, Wolters VM, Adriaanse MP, Van den Neucker AM, van Bijnen AA, Houwen R, et al. Additional value of serum I-FABP levels for evaluating celiac disease activity in children. Scand J Gastroenterol. 2011;46(12):1435-41. 
23. Lau E, Marques C, Pestana D, Santoalha M, Carvalho D, Freitas P, et al. The role of I-FABP as a biomarker of intestinal barrier dysfunction driven by gut microbiota changes in obesity. Nutr Metab (Lond). 2016;13:31.

24. Piton G, Belon F, Cypriani B, Regnard J, Puyraveau M, Manzon C, et al. Enterocyte damage in critically ill patients is associated with shock condition and 28-day mortality. Crit Care Med. 2013;41(9):2169-76.

25. Zou L, Song X, Hong L, Shen X, Sun J, Zhang C, et al. Intestinal fatty acid-binding protein as a predictor of prognosis in postoperative cardiac surgery patients. Medicine (Baltimore). 2018;97(33):e11782.

26. Dohle DS, Bestendonk C, Petrat F, Tsagakis K, Wang M, Strucksberg KH, et al. Serum markers for early detection of patients with mesenteric ischemia after cardiac surgery. Innov Surg Sci. 2018;3(4):277-83.

27. Camara-Lemarroy CR, Escobedo-Zuniga N, Guzman-de la Garza FJ, Castro-Garza J, Vargas-Villarreal J, Gongora-Rivera F. D-Lactate and intestinal fatty acid-binding protein are elevated in serum in patients with acute ischemic stroke. Acta Neurol Belg. 2018.

28. Ho SW, Chang SC, Chen LW. Urinary Intestine Fatty Acid Binding Protein is Associated with Poor Outcome of Pneumonia Patients in Intensive Care Unit. Clin Lab. 2016;62(11):2219-26.

29. Dutta D, Methe B, Amar S, Morris A, Lim SH. Intestinal injury and gut permeability in sickle cell disease. J TransI Med. 2019;17(1):183.

30. Kastl SP, Krychtiuk KA, Lenz M, Distelmaier K, Goliasch G, Huber K, et al. Intestinal Fatty Acid Binding Protein is Associated With Mortality in Patients With Acute Heart Failure or Cardiogenic Shock. Shock. 2019;51(4):410-5.

31. Kartaram S, Mensink M, Teunis M, Schoen E, Witte G, Janssen Duijghuijsen L, et al. Plasma citrulline concentration, a marker for intestinal functionality, reflects exercise intensity in healthy young men. Clin Nutr. 2019;38(5):2251-8.

32. Quigley EM. Gut bacteria in health and disease. Gastroenterol Hepatol (N Y). 2013;9(9):560-9.

33. Wouters EFM. The lung-gut-cross talk in chronic airways diseases. Barcelona respiratory Network reviews. 2019;5(1):19-34.

34. Jiang W, Wu N, Wang X, Chi Y, Zhang Y, Qiu X, et al. Dysbiosis gut microbiota associated with inflammation and impaired mucosal immune function in intestine of humans with non-alcoholic fatty liver disease. Sci Rep. 2015;5:8096.

35. Shin NR, Whon TW, Bae JW. Proteobacteria: microbial signature of dysbiosis in gut microbiota. Trends Biotechnol. 2015;33(9):496-503.

36. Larsen N, Vogensen FK, van den Berg FW, Nielsen DS, Andreasen AS, Pedersen BK, et al. Gut microbiota in human adults with type 2 diabetes differs from non-diabetic adults. PLoS One. 2010;5(2):e9085.

37. Vatanen T, Kostic AD, d'Hennezel E, Siljander H, Franzosa EA, Yassour M, et al. Variation in Microbiome LPS Immunogenicity Contributes to Autoimmunity in Humans. Cell. 2016;165(6):1551.

38. Rezaee F, Georas SN. Breaking barriers. New insights into airway epithelial barrier function in health and disease. Am J Respir Cell Mol Biol. 2014;50(5):857-69.

39. Aghapour M, Raee P, Moghaddam SJ, Hiemstra PS, Heijink IH. Airway Epithelial Barrier Dysfunction in Chronic Obstructive Pulmonary Disease: Role of Cigarette Smoke Exposure. Am J Respir Cell Mol Biol. 2018;58(2):157-69.

40. Ramezanpour M, Moraitis S, Smith JL, Wormald PJ, Vreugde S. Th17 Cytokines Disrupt the Airway Mucosal Barrier in Chronic Rhinosinusitis. Mediators Inflamm. 2016;2016:9798206.

41. Saatian B, Rezaee F, Desando S, Emo J, Chapman T, Knowlden S, et al. Interleukin-4 and interleukin-13 cause barrier dysfunction in human airway epithelial cells. Tissue Barriers. 2013;1(2):e24333.

42. Oelsner EC, Balte PP, Grams ME, Cassano PA, Jacobs DR, Barr RG, et al. Albuminuria, Lung Function Decline, and Risk of Incident Chronic Obstructive Pulmonary Disease. The NHLBI Pooled Cohorts Study. Am J Respir Crit Care Med. 2019;199(3):321-32.

43. de Kort S, Keszthelyi D, Masclee AA. Leaky gut and diabetes mellitus: what is the link? Obes Rev. 2011;12(6):449-58.

44. Peschel T, Schonauer M, Thiele H, Anker SD, Schuler G, Niebauer J. Invasive assessment of bacterial endotoxin and inflammatory cytokines in patients with acute heart failure. Eur J Heart Fail. 2003;5(5):609-14. 
45. Teixeira TF, Souza NC, Chiarello PG, Franceschini SC, Bressan J, Ferreira CL, et al. Intestinal permeability parameters in obese patients are correlated with metabolic syndrome risk factors. Clin Nutr. 2012;31(5):735-40.

46. Wiedermann CJ, Kiechl S, Dunzendorfer S, Schratzberger P, Egger G, Oberhollenzer F, et al. Association of endotoxemia with carotid atherosclerosis and cardiovascular disease: prospective results from the Bruneck Study. J Am Coll Cardiol. 1999;34(7):1975-81.

47. Kiechl S, Lorenz E, Reindl M, Wiedermann CJ, Oberhollenzer F, Bonora E, et al. Toll-like receptor 4 polymorphisms and atherogenesis. N Engl J Med. 2002;347(3):185-92.

48. Agusti A, Edwards LD, Rennard SI, MacNee W, Tal-Singer R, Miller BE, et al. Persistent systemic inflammation is associated with poor clinical outcomes in COPD: a novel phenotype. PLoS One. 2012;7(5):e37483.

49. van Wijck K, Verlinden TJ, van Eijk HM, Dekker J, Buurman WA, Dejong CH, et al. Novel multi-sugar assay for site-specific gastrointestinal permeability analysis: a randomized controlled crossover trial. Clin Nutr. 2013;32(2):245-51. 



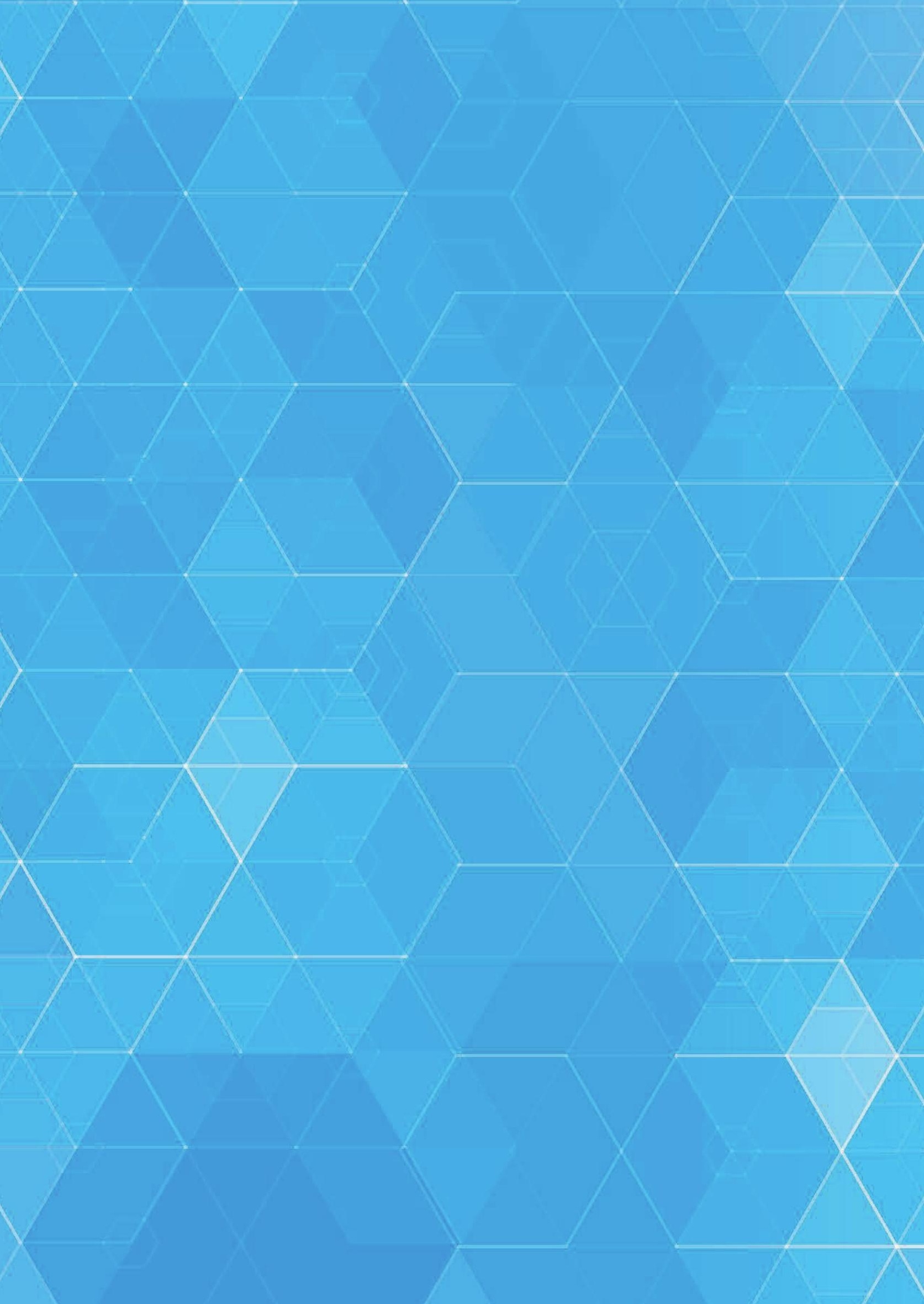




\title{
Chapter 10
}

\author{
General discussion
}





\section{Introduction}

Chronic obstructive pulmonary disease (COPD) is one of the most prevalent noncommunicable diseases globally. ${ }^{1}$ It accounted for more than half of chronic respiratory disease prevalence in $2017^{1}$ and is accompanied by significant morbidity and mortality. ${ }^{2}$ The Global Burden of Disease 2015 study reported that COPD was the third leading cause of death worldwide, after ischemic heart disease and stroke ${ }^{3}$, with an increasing trend due to population growth and ageing. In 2017, 41.9 deaths per 100.000 individuals, or $5.7 \%$ of total all-cause deaths, were attributable to this disease. ${ }^{1}$ In addition, COPD is the major respiratory cause of disability-adjusted life-years ${ }^{1}$ and is associated with tremendous social and economic burden. ${ }^{4}$

COPD is defined by the presence of persistent respiratory symptoms and airflow limitation, resulting from a mixture of small airways disease and parenchymal destruction. ${ }^{5}$ The relative contributions of these pathologies vary from patient to patient. COPD results from the complex interaction between genetic susceptibility and the environment. Cigarette smoking is the most important environmental risk factor for the disease; exposure to other noxious particles or gases contributes too. ${ }^{5}$ Furthermore, there is increasing evidence that abnormal lung development in early life and childhood contributes to COPD pathophysiology in a substantial proportion of patients. ${ }^{6}$ Dyspnoea, cough and/or sputum production are the most frequent symptoms experienced by patients with this disease, limiting them in daily life. However, in the last decades, it became clear that the burden of disease for patients with COPD poorly correlates with the degree of airflow limitation. ${ }^{7}$ Moreover, the physiologic variable used to grade the severity of pulmonary impairment in COPD, the forced expiratory volume in one second $\left(\mathrm{FEV}_{1}\right)$, does not adequately predict risk of death from respiratory or any cause in this disease. ${ }^{8,9}$ Evidently, other features of the disease including a high degree of breathlessness ${ }^{10}$, poor exercise tolerance ${ }^{11}$, low body weight and fat-free mass ${ }^{12}$, the presence of hypoxemia or hypercapnia ${ }^{13}$ and comorbidities ${ }^{14}$ are also associated with disease burden and increased risk of death in COPD.

\section{Exacerbations of COPD}

Another key factor associated with COPD morbidity and mortality is the occurrence of exacerbations of the disease. ${ }^{15}$ In the 2021 Global initiative for chronic Obstructive Lung Disease (GOLD) strategy document, an exacerbation is defined as an acute worsening of 
respiratory symptoms that results in additional therapy. ${ }^{5}$ Increased dyspnoea is the key symptom of an exacerbation. Other symptoms include increased sputum purulence and volume, together with increased cough and wheeze. ${ }^{15}$ Patients with frequent exacerbations have accelerated lung function decline ${ }^{16}$, worse quality of life ${ }^{17}$, are at increased risk of cardiovascular events ${ }^{18}$ and have greater mortality. ${ }^{19}$ Also, exacerbations account for the majority of COPD-related healthcare costs. ${ }^{4}$ Exacerbations are very heterogeneous events in terms of frequency of occurrence ${ }^{20}$, timing of onset ${ }^{21}$, triggers ${ }^{22}$ and severity. ${ }^{23}$ Regarding the latter, event-based criteria are currently used, i.e. based on increased use of healthcare services (increased use of reliever medication, or treatment with systemic corticosteroids/antibiotics or hospitalisation) in the presence of acute worsening condition of the patient. ${ }^{24}$

The majority of exacerbations are graded as mild or moderate ${ }^{25}$, with less than $10 \%$ of these events resulting in hospital admission (severe exacerbation). A higher degree of lung function impairment ${ }^{20}$, right heart failure ${ }^{26}$ and diabetes ${ }^{27}$ are among the risk factors for severe exacerbations. ${ }^{28}$ Although a severe exacerbation is defined as an event requiring hospitalization, there are no objective indications for this. Severe symptoms (sudden worsening of dyspnea at rest, high respiratory rate, decreased oxygen saturation, confusion, drowsiness), acute respiratory failure, new physical signs such as edema or cyanosis, failure to respond on initial medical treatment, presence of serious (cardiovascular) comorbidities and insufficient home support are potential indications for hospitalization. ${ }^{5}$ The lack of objective criteria, results in a large variation in demographic, clinical and biochemical features of COPD patients hospitalized with exacerbation. Also, the heterogeneity of this population makes it difficult to predict short- or longer-term outcomes for individual patients. To further assess the acute risk within patients with severe exacerbations, patients are classified into those with and without acute respiratory failure, based on clinical signs, blood gas analysis and response to supplemental oxygen therapy. ${ }^{5}$ Biochemically, respiratory failure is defined in terms of hypoxemia and/or hypercapnia, resulting from a combination of respiratory insults including airways inflammation, increased airflow limitation and air trapping ${ }^{15}$, respiratory muscle dysfunction ${ }^{29}$ and ventilation/perfusion mismatch. ${ }^{30}$ Indeed, approximately $20 \%$ of patients hospitalised for COPD present with ${ }^{31}$ or develop hypercapnic respiratory failure ${ }^{32}$, which is an indicator of an increased risk of death. ${ }^{33}$ Acute non-invasive ventilation (NIV) has been shown to improve outcome in acute lifethreatening hypercapnic respiratory failure in COPD. ${ }^{34}$ Endotracheal intubation and invasive mechanical is used for those with deterioration on NIV or who are no candidates for this intervention. Predictors for short- and longer-term outcomes in 
severe exacerbations with acute respiratory failure are mostly unknown and could be used for clinical decision support on emergency or pulmonary wards for COPD patients.

Mortality related to severe exacerbations is particularly high during hospitalization, and the months thereafter. ${ }^{35}$ Different studies reported a wide range of mortality rates of hospitalized COPD patients, probably related to the previously described heterogeneity of these events and organization and standard of healthcare. ${ }^{36-39}$ Also, readmissions are common $^{40,41}$ and are associated with worse outcomes. Over the last decade, long-term home NIV is increasingly used to improve health outcomes by targeting chronic hypercapnia in COPD patients with persistent hypercapnic respiratory failure, although limitations of evidence are recognized. ${ }^{42}$ Identifying patients who are either at increased risk or respond well to specific interventions is challenging but relevant in daily clinical practice and for developing novel treatments that may improve outcomes by targeting the treatable traits ${ }^{43}$ underlying them.

While it is well-recognized that COPD exacerbations are often complicated by acute cardiac dysfunction ${ }^{44}$, the impact of these events on other organs is scarcely studied. Especially, the interaction between gastro-intestinal $(\mathrm{Gl})$ and the respiratory tract is of pathophysiologic interest. Increased GI permeability and enterocyte damage were observed in stable COPD compared to controls, following performance of activities of daily living in a small study. ${ }^{45}$ Potentially, this was related to exercise-induced oxygen desaturation that is commonly observed in normoxemic stable COPD. ${ }^{46}$ Thus, it can be hypothesized that hypoxemic respiratory failure during severe exacerbation, may cause GI disruption and microbial dysbiosis. ${ }^{47}$ Vice versa, changes in gut microbiota may be linked to altered systemic and pulmonary immunity, especially when the gut barrier function is reduced ${ }^{48}$ and bacterial translocation across the intestinal wall may occur.

In summary, severe exacerbations and especially those with acute respiratory failure form the tip of the iceberg of the total number of exacerbations in COPD. This tip is characterized by pathophysiologic, clinical and biochemical heterogeneity, which remains largely covered. Despite the tremendous personal and economic burden of these severe exacerbations, their management is still largely experience-based and outcome is variable and unpredictable. Also, the systemic impact of these events, especially the interactions between GI tract and the lungs, warrants further study.

This thesis aimed to investigate short- and longer-term mortality rates for COPD patients hospitalized for severe exacerbations with or without acute respiratory failure. Also, investigating predictors for poor outcomes and studying the outcomes of NIV in 
the acute and post-acute phase of severe exacerbations were goals of the thesis. Finally, the thesis investigated gastro-intestinal function in relation to exacerbations of COPD. Results of the thesis are discussed here and directions for further study are provided.

\section{Prognosis of severe COPD exacerbations}

Several epidemiologic cohort studies reported that COPD is associated with reduced prognosis. ${ }^{49,50}$ There are several limitations in these epidemiologic observations. First, the diagnosis COPD is frequently based on pre-bronchodilator spirometry, potentially including those with significant bronchodilator response and not in accordance with the GOLD definition of COPD. ${ }^{5}$ Also, as the diagnosis of COPD requires the presence of chronic respiratory symptoms, inclusion of subjects with only obstructive spirometry may result in selection bias towards asymptomatic or less severe cases. Third, detailed patient characteristics and information on treatments is mostly lacking in epidemiologic studies. Also, most population-based studies included subjects with mild-to-moderate airflow limitation; thus, patients with severe disease are underrepresented. Finally, most longitudinal studies included COPD patients from the general population, while long-term outcomes of those hospitalized for exacerbations remain understudied. In the current thesis, four chapters studied mortality following severe COPD exacerbations in different cohorts, confirming the detrimental impact of these events. The mortality rates at different durations of follow up in the four chapters are summarized in Figure 10.1 .

In chapter 3, a large population of GOLD-diagnosed patients admitted to the hospital with a clinical diagnosis of COPD exacerbation was followed for 15 years and mortality risk was compared to sex and age matched controls from the general population in the same region. After 5 years of follow up, more than half of patients had died, compared to approximately one quarter of controls. After 10 years, $72 \%$ of patients had died and $>80 \%$ had after 15 years. The study confirmed that life expectancy following severe exacerbations is tremendously reduced in comparison to general population. Also, it showed that spirometric GOLD stages have significant prognostic capacity in this population, although previous studies suggested that $\mathrm{FEV}_{1}$ is a poor predictor of mortality. ${ }^{51}$ Importantly, the study showed that survival of patients with GOLD stage 1 was not reduced compared to general population and that mainly smoking status, one of the treatable traits in COPD ${ }^{43}$, especially influenced survival in that group. Strengths of this study included the large proportion of patients with severe airflow limitation, 
their detailed characterization and the long follow up duration. There was no recording of in-hospital mortality in this chapter as follow-up started after hospitalization. Chapter 4 recruited hospitalized patients from another hospital (Maastricht UMC+) and a decade later than the previous chapter. In-hospital mortality was lowest of the three chapters in this thesis that assessed this outcome (Figure 10.1). This probably results from the lower percentage of patients with acute (on chronic) respiratory insufficiency in this cohort, as the results of this thesis clearly show that patients requiring NIV have higher in-hospital mortality (Figure 10.1). Also, after 90 days and 1 year, mortality in those who required NIV during hospitalization (chapter 6) was substantially higher than in a less severe COPD population (chapters 4 and 5). As shown in Figure 10.1, 2-year mortality of patients that required NIV was actually higher than 5-year mortality of the patients in chapter $\mathbf{3}$, who had a comparable age and body mass index (BMI) but a better lung function at baseline. To conclude, the results of this thesis consistently show that severe exacerbations are associated with high short- and long-term mortality and that both the severity of underlying COPD as well as the presence of acute (on chronic) respiratory failure and need for NIV contribute to increased mortality.

Figure 10.1 Mortality in hospitalized COPD exacerbations in this thesis.

- Chapter 3: Hospitalized COPD

- Chapter 4: Hospitalized COPD

$\times$ Chapter 5: Hospitalized COPD, $30 \%$ requiring NIV

v Chapter 6: Hospitalized COPD, $100 \%$ requiring NIV

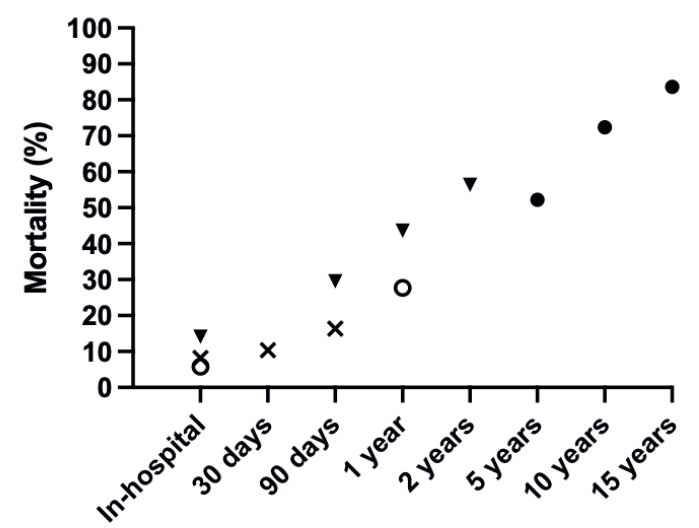

Follow-up period 


\section{Hospitalisations in less advanced COPD}

An important observation in this thesis is the high frequency of patients with mild or moderate airflow limitation within the studied populations of hospitalized exacerbations. In chapter 3, more than $40 \%$ of patients had GOLD 1 or 2 ; in chapter 4 this percentage was $37 \%$, in chapter 5 it was 35\%. Also, in chapter $\mathbf{6}$, including patients requiring NIV for the first time, this percentage was $20 \%$. While it is well-established that the severity of airflow limitation is a poor predictor of patient-related outcomes in $\mathrm{COPD}^{7}$, GOLD stage may also underestimate the severity of emphysema, the degree of static and dynamic hyperinflation and the degree of mismatch in ventilation to perfusion ratio. In stable COPD and during exacerbations, the ventilation-perfusion mismatch (uneven distribution of both alveolar ventilation and pulmonary blood flow) is one of the most important causes of acute or chronic respiratory failure. ${ }^{52} \mathrm{~A}$ comprehensive study of pulmonary gas exchange disturbance across the GOLD stages for airflow limitation reported that the pulmonary gas exchange is disproportionately abnormal in GOLD Stage 1, which increasingly progressed as lung function became worse. $^{52}$ This highlights the need to create awareness among healthcare professionals that also patients with at first sight mild or moderate COPD may develop acute respiratory failure. It also stresses the importance of comprehensive lung function assessment in COPD $^{53}$, especially in patients with discordance between the degree of airflow limitation and burden of disease.

\section{Differential diagnosis of severe exacerbations}

As mentioned earlier, nowadays, it's well-recognized that exacerbations are heterogeneous events that must be differentiated from other acute pulmonary or extra-pulmonary conditions with overlapping symptoms before they can be diagnosed. ${ }^{54}$ Indeed, the frequency of acute heart failure in COPD patients at the emergency department is $20-30 \%{ }^{55,56}$, while the prevalence of pulmonary embolism in COPD hospitalized with acutely worsening respiratory symptoms is $5.9 \%{ }^{57}$ This precision approach towards exacerbations was introduced over the last years. ${ }^{58}$ The cohorts of hospitalized COPD patients in this thesis however, were formed $>20$ years ago (chapter $\mathbf{3}$ ), >10 years ago (chapter $\mathbf{4}$ ), 5-10 years ago (chapters 5 and 6 ), when there was less clinical awareness of the clinical conditions that could mimic or aggravate COPD exacerbations. Indeed, almost one-third of patients with a severe exacerbation in chapter $\mathbf{4}$ had radiologic evidence of pneumonia and thus would not fulfil diagnostic criteria for exacerbation in $2021 .^{5,54}$ Still, the prevalence of pneumonia 
was similar in patients who died by year 1 and those who were alive and pneumonia was not identified as an independent risk factor for mortality in chapter $\mathbf{3}$. Similar observations regarding frequency of pneumonia and mortality were reported in chapter $\mathbf{5}$. This suggests that the observations in chapters $\mathbf{3}$ and $\mathbf{5}$ are robust. Twothirds of patients in chapter 3 had a medical history of cardiovascular comorbidity, but since decompensated heart failure, myocardial infarction, arrhythmia and pulmonary embolism were not proactively ruled out in all patients, their contribution to in-hospital and 1-year mortality cannot be excluded. The facts that priorly recorded congestive heart failure was one of the identified independent predictors of 1-year mortality in chapter 3 and that deceased patients had a numerically higher percentage of pulmonary oedema on chest X-ray at presentation are in line with potential underdiagnosis of this condition in the acute event. In chapter 5, more blood biomarkers were available for differential diagnosis; increased NT-proBNP (a biomarker for acute heart failure) and increased Troponin (a biomarker for cardiac ischemia) were observed in $22 \%$ and $25 \%$ of patients, respectively. Although these biomarkers did not contribute to the CART model for risk stratification, the presence of atrial fibrillation was three times higher in patients who died during hospital stay or who died within 90 days. This emphasizes the clinical impact of cardiovascular comorbidity in COPD. In chapter 6, including the most severe exacerbating patients requiring NIV, outcomes were mainly driven by respiratory factors including duration of NIV need and response to the intervention. Also, respiratory failure was the cause of death of half of patients that died during follow-up in chapter 6 and in who a cause of death was established. This is in line with other studies, indicating that comorbidities contribute relatively less to mortality in those with severe to very severe airflow limitation. ${ }^{59}$

Blood eosinophilia is increasingly recognized as a biomarker for response to inhaled corticosteroids (ICS) ${ }^{60,61}$, for risk of pneumonia associated with ICS in COPD ${ }^{62}$ and for risk of exacerbations. ${ }^{63}$ In addition, it was recently shown that patients with severe eosinophilic exacerbations had shorter length of hospital stay that those with noneosinophilic exacerbations. ${ }^{64}$ No difference in in-hospital or 1-year mortality was observed between eosinophilic and non-eosinophilic patients. In this thesis, blood eosinophils were recorded in chapter $\mathbf{5}$ only. The observation that eosinophils percentage was significantly increased in patients alive at hospital discharge and at year 1 , compared to those deceased, is in line with the previous study, suggesting that blood eosinophilia in COPD is associated with better outcomes. 


\section{Predictors for mortality after hospitalised COPD exacerbations}

In addition to increasing the understanding of short- and long-term mortality rates following severe exacerbations, several chapters of this thesis investigated prognostic factors after hospitalizations. An overview of the identified factors in the different chapters is included in Table 10.1. Identification of these factors may provide opportunities to alter the course of the disease, as long as these factors are modifiable.

Table 10.1 Overview independent predictors mortality following hospitalized COPD exacerbation in the various chapters of the thesis.

\begin{tabular}{lcccc}
\hline Predictor & Ch3 & Ch4 & Ch5 & Ch6 \\
\hline Age & $\mathrm{X}$ & $\mathrm{X}$ & $\mathrm{X}$ & $\mathrm{X}$ \\
Male sex & $\mathrm{X}$ & $\mathrm{X}$ & & \\
BMI & $\mathrm{X}$ & & $\mathrm{X}$ & \\
Low FEV & & & \\
Low diffusion capacity & $\mathrm{X}$ & & & \\
Hypercapnia & $\mathrm{X}$ & $\mathrm{X}$ & $\mathrm{X}$ & \\
Prior hospitalisations & $\mathrm{X}$ & $\mathrm{X}$ & $\mathrm{X}$ & \\
Re-admissions & & $\mathrm{X}$ & & \\
Elevated urea & $\mathrm{X}$ & $\mathrm{X}$ & & \\
Comorbid heart failure & $\mathrm{X}$ & & $\mathrm{X}$ \\
Charlson Comorbidity Index & & & & $\mathrm{X}$ \\
NIV use $>8$ days & & & & \\
Not successful response to NIV & &
\end{tabular}

$\mathrm{BMI}$ : body mass index; $\mathrm{FEV}_{1}$ : forced expiratory volume in the first second; NIV: non-invasive ventilation.

Older age was consistently identified as an independent risk factor for mortality in this thesis. In fact, several studies previously reported this risk factor in COPD patients following hospitalization in general ${ }^{37,65}$, in patients hospitalized for first-ever ${ }^{66}$ and in the subgroup of ICU-admitted patients. ${ }^{67}$ Age is one of the variables in one of the most commonly used prognostic indices in stable COPD, the Age-Dyspnoea-airflow Obstruction (ADO) index $^{68}$ and several risk scores for mortality following hospitalisations included this factor, including $\mathrm{APACHE}^{69}, \mathrm{PEARL}^{70}$ and CURB65. ${ }^{71}$ Thus, the results of this thesis confirm the relevance of age as a risk factor for mortality in hospitalized COPD patients. Rather than a factor to be taken into account in clinical decision making, age obviously is not a directly modifiable factor. Some aging-related alterations, including loss of skeletal muscle mass ${ }^{72}$ and chronic systemic inflammation ${ }^{73}$, however, may be accessible for intervention. Indeed, interleukin (IL)-6, tumor necrosis factor (TNF)- $\alpha$, and their receptors, are upregulated in aged tissues and cells. ${ }^{74}$ Elevated levels of chemokines and C-reactive protein (CRP) have been found to be involved in age-related pathogenesis. ${ }^{75}$ In a proportion of patients with COPD, these 
inflammatory markers are persistently elevated and associated with increased all-cause mortality. ${ }^{76}$ Also, COPD is increasingly recognized as a disease of accelerated aging. ${ }^{77}$ Telomere length and gene expression of anti-aging proteins sirtuin-1 and soluble klotho and DNA repair protein Ku70 were significantly reduced in COPD patients compared to smoking controls, while senescence protein p21 gene expression was increased. ${ }^{78}$ Thus, although it is unrealistic to achieve reversal of normal aging, development of senescence drugs in COPD is a field of growing scientific interest and may lead to fine tuning the pathways that result in premature senescence with potential to prolong survival. $^{79}$

Gender differences in COPD have gained a lot of attention over the past decade. While COPD was traditionally considered to be a disease that mainly affected elderly men, it is now equally common in females. ${ }^{1}$ In fact, females appear to have more severe COPD with early-onset disease, a faster annual decline in lung function ${ }^{80}$ and a greater susceptibility to COPD with lower tobacco exposure. ${ }^{81}$ Also, females with COPD more frequently have psychological comorbidities and this is associated with increased burden of disease. ${ }^{82}$ Female COPD patients also have a $50 \%$ increased risk of hospitalization or death for COPD compared to males. ${ }^{83}$ Although COPD-related mortality and ventilatory impairment associated with smoking have been found to be consistently higher among females than among males ${ }^{84}$, several prognostic studies focussing on post-hospitalization in COPD, reported male gender as an independent risk factor. $^{38,85,86}$ The latter was confirmed in this thesis (chapters 3 and 4). Although the cause of this apparent discrepancy is unclear, the increasing proportion of male patients from GOLD 1 (39\%) to GOLD 4 (65\%) and potentially other residual disease severity may have confounded this observation. Future studies are warranted to investigate potential gender differences in histologic pattern ${ }^{87}$, response to inhaled medications ${ }^{88}$ and long-term oxygen therapy ${ }^{89}$ that may contribute to differential prognosis for males and females with COPD.

Although low BMI is associated with poor survival in stable COPD $^{90}$, the results of chapters 3, 4 and 6 showed that BMI was no independent predictor of mortality. This contrasts with a study by Lainscak et al., ${ }^{91}$, who reported increased mortality during $>3$ years follow up in low BMI patients following hospitalization for severe exacerbation. ${ }^{91}$ Although the mean age of the COPD patients in the study by Lainscak et al. ${ }^{91}$ was similar to that of the COPD patients in chapters $\mathbf{3}$ and $\mathbf{4}$, differences in disease severity may contribute to the discrepancy; it is well-known that the association between low $\mathrm{BMI}$ and mortality risk is more pronounced in patients with more severe airflow limitation. ${ }^{90}$ In the classification and regression tree analysis in chapter 5, low BMI was 
indeed identified as an independent predictive factor in a COPD population with a high frequency of need for acute mechanical ventilation (as a proxy for more severe disease). Then again, in chapter 6 , which included only patients requiring non-invasive ventilation for the treatment of acute respiratory failure for the first time in the course of their disease, BMI was only univariately related to in-hospital and long-term mortality. Unrecorded differences in the disease trajectories ${ }^{92}$ or activity ${ }^{93}$, triggers for exacerbation ${ }^{22}$ or co-occurring conditions ${ }^{94}$ may have contributed to this discrepancy. Also, undiagnosed differences in body composition, i.e. muscle mass, between patients in the different chapters may contribute to the observed variation, as it is known that body composition is a stronger predictor of mortality in COPD than BMI. ${ }^{12}$

Hypercapnia is another consistent risk factor for mortality in this thesis. In COPD patients, it is the consequence of ventilatory failure resulting from a combination of severe airflow limitation, lung hyperinflation, respiratory muscle insufficiency and alterations in central ventilatory control. Chronic hypercapnic respiratory failure is inversely associated with overall prognosis $^{95}$ and with mortality following hospitalizations ${ }^{37,96}$ in COPD. However, $\mathrm{PaCO}_{2}$ levels were not included in most of the well-known predictive scores following hospitalizations. Instead, these predictive models such as the DECAF score ${ }^{97}$ included acidemia as a surrogate. In most studies, dyspnoea or use of accessory inspiratory muscles was used as predictor for mortality. These parameters indicate respiratory distress or reflect respiratory failure. Alternatively, high levels of $\mathrm{PaCO}_{2}$ reflect decreased alveolar ventilation, indicating very severe respiratory pump failure in acute patients characterized by dyspnoea and use of their accessory respiratory muscles. Since non-invasive positive pressure ventilation was identified as an effective intervention in chronic hypercapnic COPD ${ }^{42}$ by improving carbon dioxide and oxygen gas exchange, hypercapnia is considered a treatable trait of the disease. ${ }^{43}$

The association between the degree of impairment in $\mathrm{FEV}_{1}$ and mortality risk following hospitalization may seem obvious, as $\mathrm{FEV}_{1}$ is the traditional marker of disease severity ${ }^{98}$ and is included in many prognostic indices of the disease, including BODE ${ }^{51}$ and ADO. ${ }^{68}$ However, the results of this study indicated that also low diffusion capacity is an independent risk factor for mortality. It is increasingly recognized that pathophysiology of COPD is far more complex than airflow limitation; significant lung hyperinflation and/or diffusion impairment may occur in patients with mild to moderate COPD. In fact, based on extensive pulmonary function testing, seven different lung function clusters were identified in patients with mild to very severe COPD. ${ }^{99}$ These clusters poorly predicted functional performance and health status ${ }^{99}$, but there association with 
mortality risk is currently unexplored. Also, in smokers without COPD, reduced diffusion capacity is increasingly recognized as an early marker of small airways disease, a precursor lesion to emphysema. ${ }^{100}$ Within a cohort of active smokers, $22 \%$ of subjects with normal $\mathrm{FEV}_{1}$ but reduced diffusion capacity developed COPD during < 4 years follow up, compared to only $3 \%$ of the subjects with normal spirometry and normal diffusion capacity. ${ }^{101}$ The results of this thesis confirm the added value assessing diffusion capacity in COPD.

Both a history of previous hospitalizations as well as a readmission after hospitalization are well-known risk factors for mortality in COPD. ${ }^{19,36,96,102,103}$ The results of this thesis are in line with this existing literature and underscore the importance of developing strategies to reduce index hospitalizations and readmissions in COPD. ${ }^{104}$ Indeed, it is estimated that $10 \%-55 \%$ of readmissions after hospitalization for exacerbation may be preventable. ${ }^{105}$ Factors thought to contribute to early readmission include premature discharge from the hospital for the index admission, poor discharge medication reconciliation, lack of family education on disease management, and lack of communication with outpatient physicians who will be assuming future care. ${ }^{105}$ Also, co-occurring chronic heart failure, frailty, psychiatric diseases and other medical conditions are identified risk factors. ${ }^{105,106}$ A systematic review by Axson et al., ${ }^{107}$ showed that heart failure increases COPD-related hospitalizations and all-cause mortality. In comparison with COPD patients with heart failure, the risk of all-cause mortality was 1.6 times higher in those COPD patients without heart failure. Furthermore, Axson et al., reported that a postponed diagnosis of heart failure in COPD patients and under-treatment of cardiovascular conditions in these patients may contribute to increased frequency of hospital admissions and risk of death. ${ }^{107}$ Strategies that may improve readmissions after severe exacerbations include patient selfmanagement education, inhaler device training, receipt of respiratory medications prior to hospital discharge ${ }^{108}$, early follow-up, pulmonary rehabilitation ${ }^{109}$, telemonitoring ${ }^{110}$, optimal management of comorbidities ${ }^{111}$ and step-up pharmacotherapies. ${ }^{112}$ Given the heterogeneity and complexity of factors contributing to risk of readmission in individual patients, comprehensive care management programs are increasingly being used. ${ }^{113}$ As not all studies using this approach have been positive ${ }^{114}$, more research is needed to study whether these

In 2021, a novel clinical prediction tool for in-hospital of COPD patients with severe exacerbations complicated by acute (acidaemic) hypercapnic respiratory failure requiring (noninvasive) ventilation was developed and externally validated. ${ }^{115}$ Inhospital mortality was $>\mathbf{2 0} \%$ and thus substantially higher than in the current thesis, 
probably reflecting the higher severity of underlying disease and of the acute event in that study. Increased dyspnoea score, prolonged time from admission to acidaemia, low $\mathrm{pH}$, presence of atrial fibrillation, lower Glasgow coma scale and the presence of chest radiograph consolidation were identified as discriminative and contributing items to this NIV Outcomes (NIVO) score. ${ }^{115}$ The score outperformed comparator scores including APACHE $\|^{116}$, COPD and Asthma Physiology Score (CAPS) ${ }^{117}$, CURB $65^{118}$, HACOR $^{18,119}$, or DECAF. ${ }^{97}$ The performance of the CART risk stratification tool presented in chapter $\mathbf{5}$ of this thesis was not compared to NIVO score yet. Since there are no overlapping items between these tools, this is certainly interesting for future comparison. The variables included in the NIVO score also have limited overlap with the other identified predictors for poor outcomes in the present thesis.

\section{Non-invasive ventilation for persistent hypercapnia}

In patients admitted for COPD exacerbations with acute (on chronic) respiratory failure NIV is the established treatment as it has been shown to reduce mortality in the acute phase of admission. ${ }^{120,121}$ As shown by many studies and in the previous chapters of this thesis, survivors of acute life-threatening hypercapnic respiratory failure have a high risk of death in the follow-up of hospitalisation. By the time of initiation of this thesis, several studies had investigated the application of long-term nocturnal NIV in stable hypercapnic COPD. No clear benefits had been demonstrated. ${ }^{122}$ However, it was hypothesized that patients who previously required mechanical ventilation for acute respiratory failure would benefit. In chapter $\mathbf{7}$, a multi-center, randomised, controlled, parallel-group study was performed to investigate the effectiveness of long-term nocturnal NIV in patients with COPD with prolonged hypercapnia after ventilatory support for acute respiratory failure. A large population of exacerbating patients hospitalised with acute respiratory failure and with hypercapnia $>48$ hours after ending treatment with mechanical ventilation, underwent randomisation to NIV or standard care and were followed up for 1 year. The study was negative regarding its primary outcome; time to readmission for respiratory causes or death was comparable in patients randomised to NIV and to standard treatment. Also, no differences in annual number of exacerbations, lung function or health status were observed between groups. As previously described, acute respiratory failure in COPD is not restricted to those with advanced airflow limitation. No patients with GOLD stage 1 or 2 were included in chapter 7, so the results may not be extended to all COPD patients. Although mean daytime $\mathrm{PaCO}_{2}$ improved significantly more in the NIV group, $\mathrm{PaCO}_{2}$ values were comparable in both groups after 12 months. This suggested that 
spontaneous recovery of $\mathrm{PaCO}_{2}$ may occur beyond the first days after an episode of NIV and that there was a rationale to study home NIV in patients with chronic respiratory failure following an acute event. In 2014, Köhnlein et al., ${ }^{123}$ reported the results of a prospective, multicentre, randomised, controlled clinical trial including patients with stable very severe and $\mathrm{PaCO}_{2}$ of $7 \mathrm{kPa}$ or higher and $\mathrm{pH}$ higher than $7 \cdot 35$. In contrast to the study in chapter 7, there was a period of at least 4 weeks between the latest exacerbation and randomisation, resulting in increased likelihood of including chronic hypercapnic patients. Also, the inclusion criterium for $\mathrm{PaCO}_{2}$ was clearly higher than that in chapter 7 ( $\geq 7.0 \mathrm{kPa}$ vs. $>6.0 \mathrm{kPa}$ ) and IV was more aggressively targeted to reduce $\mathrm{PaCO}_{2}$. This resulted in increased mean positive inspiratory pressure (21.6 $\mathrm{cmH}_{2} \mathrm{O}(4.7)$ vs. $\left.19.2 \mathrm{cmH}_{2} \mathrm{O}(3.4)\right)$, while mean expiratory pressure $\left(4.8 \mathrm{cmH}_{2} \mathrm{O}\right.$ (1.6) vs. $\left.4.8 \mathrm{cmH}_{2} \mathrm{O}(1.0)\right)$ and backup frequency were comparable. In contrast to the study in chapter 7, the Köhnlein study showed a significant reduction in 1-year mortality in patients randomised to home NIV. In addition, NIV treatment was associated with significant improvement in blood gases, FEV $_{1}$ and overall health status. ${ }^{123}$ In 2017, Murphy et al., ${ }^{124}$ published another randomized clinical trial of COPD patients with persistent hypercapnia $\left(\mathrm{PaCO}_{2}>7.0 \mathrm{kPa}\right)$ and hypoxemia at 2-4 weeks after resolution of respiratory acidemia. As in the study by Köhnlein et al., ${ }^{123}$ patients with BMI $>35 \mathrm{~kg} / \mathrm{m}^{2}$ and obstructive sleep apnoea syndrome, or other causes of respiratory failure were excluded. Patients were randomized to home oxygen alone and to home oxygen plus home NIV (median inspiratory positive airway pressure of 24 (IQR, 22-26) $\mathrm{cmH}_{2} \mathrm{O}$, expiratory positive airway pressure of 4 (IQR, 4-5) $\mathrm{cmH}_{2} \mathrm{O}$, backup rate of 14 (IQR, 14-16) breaths/minute). The time to readmission or death within 12 months was significantly prolonged in the home NIV group, with an absolute risk reduction of $17 \% .{ }^{124}$ Also, health-related quality of life was improved. The results of the studies published after the study of chapter 7 support the screening of patients with COPD after receiving acute NIV to identify persistent hypercapnia and introduce home NIV. Indeed, in 2019, a European Respiratory Society taskforce conditionally supported the application of long-term home NIV to improve health outcome by targeting a reduction in carbon dioxide in COPD patients with persistent hypercapnic respiratory failure ${ }^{42}$ and recommended implementation of this intervention in clinical practice by practitioners that routinely care for very severe chronic hypercapnic COPD patients.

\section{Intestinal integrity and COPD exacerbations}

Many extra-pulmonary features and comorbidities have been associated with COPD, its burden of disease and prognosis, including loss of skeletal muscle mass ${ }^{125}$, loss of bone 
mineral density ${ }^{126}$, coronary artery calcification ${ }^{127}$, chronic heart failure ${ }^{128}$ and psychological conditions. ${ }^{129}$ Traditionally, the gastro-intestinal tract had attracted less scientific attention as a relevant organ in the pathophysiology of the disease, although it was reported that gastro-intestinal symptoms are common in COPD. ${ }^{130}$ In parallel to several other acute or chronic conditions however, increased permeability of the small intestine and colon and was observed in COPD compared to controls, which was further increased after performing activities of daily living and was accompanied by activityinduced enterocyte damage. ${ }^{45}$ Indeed, microbial and immune interactions between the gut and the lungs have been increasingly studied over the last decade, which has resulted in the concept of the gut-lung axis. ${ }^{131}$

In chapter $\mathbf{8}$ of this thesis, increased small intestine permeability was reported in patients with (very) severe COPD hospitalized with acute hypoxaemic respiratory failure, compared to the recovered state after this event. Although the underlying mechanisms for this observation were not studied, it was hypothesized that intestinal mucosa hypoxia, resulting from reduced intestinal blood flow and sympathetic $\operatorname{activation}^{132}$, and systemic inflammation during the severe exacerbation ${ }^{133}$, play an important role. A limitation of the study was the lack of control subjects and assessment of small intestine permeability in the stable state before the exacerbation. Thus, it cannot be excluded that other factors resulted in the reduction in small intestine permeability from hospitalization to recovery. One of these factors is the treatment with systemic corticosteroids during hospital admission, which is part of routine care. ${ }^{5}$ In patients with Crohn's disease, a condition that is also characterized by increased intestinal permeability, treatment with oral corticosteroids resulted in a $50 \%$ reduction in intestinal permeability measured by urinary lactulose/mannitol ratio. ${ }^{134}$ Furthermore, intensified use of beta-adrenergic agonists to target the increased airflow limitation during the acute event of exacerbation, may enhance sympathetic activity in COPD. It is well known that inhaled beta-2-agonists induce adverse effects, such as increased heart rate and tremor. ${ }^{135}$ This is thought to be due to spillover of the betaagonist into the systemic circulation thereby causing direct activation of beta- 2 and beta-1 receptors within the cardiovascular system. However, it was previously shown that inhaled therapy with a long-acting beta- 2 agonist does not enhance the muscle sympathetic nerve activity, which is considered the gold standard to evaluate sympathetic activity ${ }^{136}$, in patients with severe COPD. ${ }^{137}$ Also, a contribution of undiagnosed comorbid heart failure, a frequent mimicker of COPD exacerbations ${ }^{55}$, cannot be excluded since this condition was not actively ruled out on admission. Indeed, a 35\% increase in large intestinal permeability was observed in patients with chronic heart failure compared to controls. ${ }^{138}$ Thus, while the results of chapter 8 
suggest that small intestinal permeability is increased during severe exacerbations, follow-up studies are needed to elucidate the potential impact of COPD treatments or comorbid conditions on this finding.

Also, the direction of association between COPD and altered gut function remains to be established. In chapter $\mathbf{8}$, it was hypothesized that COPD-related factors result in increased intestinal permeability, but an opposite association is another possibility. The mucosal epithelial integrity has a pivotal role in preventing the entry of potentially harmful luminal particles, e.g., bacteria and their products into systemic blood compartment through transcellular and paracellular pathways. ${ }^{139}$ Disturbed intestinal integrity might result in increased permeability of the intestinal mucosal barrier and bacterial translocation. Increased intestinal permeability is associated with enhanced systemic inflammation, potentially via leakage of bacterial components into the circulation and altered immune interactions in the gut. Hence, increased intestinal permeability and subsequent systemic inflammation may be upstream in the pathophysiology of COPD exacerbations. Although this has not been studied in detail, it is known that chronic low-grade systemic inflammation is indeed a risk factor for COPD exacerbations. ${ }^{140}$ Chapter 9 of this thesis focussed on the association between gut integrity, systemic inflammation and COPD exacerbations in a well-characterized cohort of moderate to clinically stable, severe COPD patients and smoking and non-smoking controls. The study showed that the gut integrity, assessed by plasma intestinal fatty acid binding protein (I-FABP), is significantly decreased in COPD compared to controls. However, I-FABP levels were comparable between patients with and without exacerbations during 2 years follow-up. Decreased gut integrity was weakly associated with systemic inflammation in the COPD patients. Thus, a contribution of decreased gut integrity in the risk of COPD exacerbation remains speculative and the link between gut function and systemic inflammation in COPD needs further study.

\section{Clinical implications and future directions}

The results of this thesis emphasize the high mortality risk following severe COPD exacerbations with or without acute (on chronic) respiratory failure. Also, several independent risk factors for mortality were identified, complementing previous studies in this field. The predictive value of these factors indicates the need for a thorough standardised evaluation of patients hospitalized for severe exacerbations, in order to guide clinicians in shared and clinical decision making and adequate disease management. In addition, in this thesis it was reported that initiation of nocturnal NIV 
in hypercapnic patients shortly following weaning from acute mechanical ventilation does not improve outcomes. Following the publication of the latter findings, highintensity home mechanical ventilation was introduced as an effective intervention in severe COPD patients with prolonged hypercapnia. ${ }^{42}$ However, not all patients respond well to this intervention, which may be related to non-compliance, failure of the intervention and/or patient selection. Regarding the latter, the different randomized trials indicated that patients with persistent chronic hypercapnia ${ }^{123,124}$ respond better to NIV than patients with post-acute residual hypercapnia which may show spontaneous recovery over time (chapter 7). Failure of the intervention may be related to the settings of NIV, the degree of hyperinflation and respiratory muscle fatigue, subsequent adaptations in respiratory drive or pulmonary mechanics and the potential impact of NIV on cardiac function. Also in responders, it remains largely unknown how NIV impacts on the respiratory system and which mechanisms are involved in the improvement in outcomes; improved health status with NIV may not be related to reduced hypercapnia, especially in patients with moderate respiratory failure. ${ }^{141}$ Alternatively, NIV may facilitate a more active lifestyle and thereby have a positive effect on health status. ${ }^{142}$ Additional studies are warranted to improve knowledge about how NIV exactly works on the pulmonary and extra-pulmonary systems and will contribute to improved outcomes in more patients who have this treatment. At the same time, novel interventions for chronic respiratory failure in COPD have been proposed over the last years for patients who do cannot tolerate or have contraindications to NIV. High-flow nasal cannula (HFNC) oxygen therapy is the first of these interventions, delivering humidified, heated oxygen-enriched air via a nasal cannula at high flow rates ${ }^{143}$; HFNC provides a washout effect of the upper airway dead space, which reduces hypercapnia. Additionally, it improves mucociliary clearance, reduces airway resistance and, consequently, the work of breathing. Finally, it delivers low level positive airway pressure, resulting in increased alveolar recruitment and improvement in gas exchange. Several studies have examined the use of HFNCs in stable COPD patients with chronic respiratory failure. Storgaard et al. ${ }^{144}$ randomized COPD patients with chronic hypoxemic respiratory failure who were already on longterm oxygen therapy (LTOT) to receive either HFNC in addition to LTOT or LTOT alone for one year. No difference in all-cause mortality or hospital admissions was observed between the groups, although the study found a decrease in patient-reported acute exacerbation rates in the HFNC group. Also dyspnoea symptoms, quality of life, $\mathrm{PaCO}_{2}$, and exercise capacity all significantly improved in the HFNC group compared to the LTOT group. ${ }^{144}$ In COPD patients with chronic hypercapnic respiratory failure, Bräunlich et al. ${ }^{145}$ performed a randomized crossover study comparing HFNC to NIV, both for 6 weeks. HFNC was non-inferior in reducing $\mathrm{PaCO}_{2}$ levels compared to $\mathrm{NIV}^{145}$ suggesting 
that it can be used as an alternative to NIV, provided that patient-related outcomes are also comparable. Finally, Rea et al., ${ }^{146}$ found that using HFNC for one year resulted in fewer exacerbation days, increased time to first exacerbation and reduced exacerbation rate, compared to usual care in a mixed population of patients with COPD and bronchiectasis, or both. Future studies should further investigate the role of HFNC in stable hypercapnic COPD patients to definitively determine the effect of this therapy on hospitalization and exacerbation rates and its role as an alternative to NIV.

In 2020, another intervention aimed at reduction of chronic hypercapnia in severe COPD was proposed in a 'proof of concept' study. This study used extracorporeal $\mathrm{CO}_{2}$ removal using a polypropylene membrane lung. ${ }^{147}$ The intervention was proposed in patients enrolled in a home care NIV programme for at least 6 months, but who were not responding in terms of $\mathrm{PaCO}_{2}$ reduction to NIV. Patients underwent extracorporeal $\mathrm{CO}_{2}$ removal on a high-intensity respiratory ward for 24-hours during spontaneous breathing. Although the intervention could not be completed in a substantial proportion of participants due to mechanical adverse events, the intervention causes a reduction in $\mathrm{PaCO}_{2}$ levels between $23-47 \%$ in the patients who completed the study. ${ }^{147}$ The effect was retained for 48-96 hours after discontinuation of extracorporeal $\mathrm{CO}_{2}$ removal. This study proposed the concept of ' $\mathrm{CO}_{2}$ dialysis' in $\mathrm{COPD}$, although clinical benefits and safety of this technically-complicated intervention remain currently unknown. 


\section{References}

1. Collaborators, G.B.D.C.R.D., Prevalence and attributable health burden of chronic respiratory diseases, 1990-2017: a systematic analysis for the Global Burden of Disease Study 2017. Lancet Respir Med, 2020. 8(6): p. 585-596.

2. Lopez-Campos, J.L., W. Tan, and J.B. Soriano, Global burden of COPD. Respirology, 2016. 21(1): p. 14-23.

3. Mortality, G.B.D. and C. Causes of Death, Global, regional, and national life expectancy, all-cause mortality, and cause-specific mortality for 249 causes of death, 1980-2015: a systematic analysis for the Global Burden of Disease Study 2015. Lancet, 2016. 388(10053): p. 1459-1544.

4. Foo, J., et al., Continuing to Confront COPD International Patient Survey: Economic Impact of COPD in 12 Countries. PLoS One, 2016. 11(4): p. e0152618.

5. WHO, Global strategy for the diagnosis, management, and prevention of chronic obstructive pulmonary disease, 2021 report. 2020.

6. Rennard, S.I. and M.B. Drummond, Early chronic obstructive pulmonary disease: definition, assessment, and prevention. Lancet, 2015. 385(9979): p. 1778-1788.

7. Agusti, A., et al., Characterisation of COPD heterogeneity in the ECLIPSE cohort. Respir Res, 2010. 11: p. 122.

8. Bang, K.M., et al., The effect of pulmonary impairment on all-cause mortality in a national cohort. Chest, 1993. 103(2): p. 536-40.

9. Anthonisen, N.R., E.C. Wright, and J.E. Hodgkin, Prognosis in chronic obstructive pulmonary disease. Am Rev Respir Dis, 1986. 133(1): p. 14-20.

10. Nishimura, K., et al., Dyspnea is a better predictor of 5-year survival than airway obstruction in patients with COPD. Chest, 2002. 121(5): p. 1434-40.

11. Spruit, M.A., et al., Predicting outcomes from 6-minute walk distance in chronic obstructive pulmonary disease. J Am Med Dir Assoc, 2012. 13(3): p. 291-7.

12. Schols, A.M., et al., Body composition and mortality in chronic obstructive pulmonary disease. Am J Clin Nutr, 2005. 82(1): p. 53-9.

13. Continuous or nocturnal oxygen therapy in hypoxemic chronic obstructive lung disease: a clinical trial. Nocturnal Oxygen Therapy Trial Group. Ann Intern Med, 1980. 93(3): p. 391-8.

14. Divo, M., et al., Comorbidities and risk of mortality in patients with chronic obstructive pulmonary disease. Am J Respir Crit Care Med, 2012. 186(2): p. 155-61.

15. Wedzicha, J.A. and T.A. Seemungal, COPD exacerbations: defining their cause and prevention. Lancet, 2007. 370(9589): p. 786-96.

16. Donaldson, G.C., et al., Relationship between exacerbation frequency and lung function decline in chronic obstructive pulmonary disease. Thorax, 2002. 57(10): p. 847-52.

17. Seemungal, T.A., et al., Effect of exacerbation on quality of life in patients with chronic obstructive pulmonary disease. Am J Respir Crit Care Med, 1998. 157(5 Pt 1): p. 1418-22.

18. Reilev, M., et al., Increased risk of major adverse cardiac events following the onset of acute exacerbations of COPD. Respirology, 2019. 24(12): p. 1183-1190.

19. Soler-Cataluna, J.J., et al., Severe acute exacerbations and mortality in patients with chronic obstructive pulmonary disease. Thorax, 2005. 60(11): p. 925-31.

20. Hurst, J.R., et al., Susceptibility to exacerbation in chronic obstructive pulmonary disease. N Engl J Med. 363(12): p. 1128-38.

21. Aaron, S.D., et al., Time course and pattern of COPD exacerbation onset. Thorax, 2012. 67(3): p. 238-43.

22. Bafadhel, M., et al., Acute exacerbations of chronic obstructive pulmonary disease: identification of biologic clusters and their biomarkers. Am J Respir Crit Care Med, 2011. 184(6): p. 662-71.

23. Balcells, E., et al., Characteristics of patients admitted for the first time for COPD exacerbation. Respir Med, 2009. 103(9): p. 1293-302.

24. Trappenburg, J.C., et al., The impact of using different symptom-based exacerbation algorithms in patients with COPD. Eur Respir J, 2011. 37(5): p. 1260-8.

25. Wedzicha, J.A., et al., Indacaterol-Glycopyrronium versus Salmeterol-Fluticasone for COPD. N Engl J Med, 2016. 
26. Orea-Tejeda, A., et al., Right heart failure as a risk factor for severe exacerbation in patients with chronic obstructive pulmonary disease: Prospective cohort study. Clin Respir J, 2018. 12(12): p. 2635-2641.

27. Castan-Abad, M.T., et al., Diabetes as a risk factor for severe exacerbation and death in patients with COPD: a prospective cohort study. Eur J Public Health, 2020. 30(4): p. 822-827.

28. Montserrat-Capdevila, J., et al., Predictive Model of Hospital Admission for COPD Exacerbation. Respir Care, 2015. 60(9): p. 1288-94.

29. Mesquita, R., et al., Respiratory muscle strength during and after hospitalization for COPD exacerbation. Respir Care, 2013. 58(12): p. 2142-9.

30. Hajian, B., et al., Changes in ventilation-perfusion during and after an COPD exacerbation: an assessment using fluid dynamic modeling. Int J Chron Obstruct Pulmon Dis, 2018. 13: p. 833-842.

31. Plant, P.K., J.L. Owen, and M.W. Elliott, One year period prevalence study of respiratory acidosis in acute exacerbations of COPD: implications for the provision of non-invasive ventilation and oxygen administration. Thorax, 2000. 55(7): p. 550-4.

32. Roberts, C.M., et al., Acidosis, non-invasive ventilation and mortality in hospitalised COPD exacerbations. Thorax, 2011. 66(1): p. 43-8.

33. Jeffrey, A.A., P.M. Warren, and D.C. Flenley, Acute hypercapnic respiratory failure in patients with chronic obstructive lung disease: risk factors and use of guidelines for management. Thorax, 1992. 47(1): p. 34-40.

34. Rochwerg, B., et al., Official ERS/ATS clinical practice guidelines: noninvasive ventilation for acute respiratory failure. Eur Respir J, 2017. 50(2).

35. Roberts, C.M., et al., Clinical audit indicators of outcome following admission to hospital with acute exacerbation of chronic obstructive pulmonary disease. Thorax, 2002. 57(2): p. 137-141.

36. Almagro, P., et al., Mortality after hospitalization for COPD. Chest, 2002. 121(5): p. 1441-8.

37. Groenewegen, K.H., A.M. Schols, and E.F. Wouters, Mortality and mortality-related factors after hospitalization for acute exacerbation of COPD. Chest, 2003. 124(2): p. 459-67.

38. Patil, S.P., et al., In-hospital mortality following acute exacerbations of chronic obstructive pulmonary disease. Arch Intern Med, 2003. 163(10): p. 1180-6.

39. Wang, Q. and J. Bourbeau, Outcomes and health-related quality of life following hospitalization for an acute exacerbation of COPD. Respirology, 2005. 10(3): p. 334-40.

40. Shah, T., et al., Understanding why patients with COPD get readmitted: a large national study to delineate the Medicare population for the readmissions penalty expansion. Chest, 2015. 147(5): p. 1219-1226.

41. Sharif, R., et al., Predictors of early readmission among patients 40 to 64 years of age hospitalized for chronic obstructive pulmonary disease. Ann Am Thorac Soc, 2014. 11(5): p. 685-94.

42. Ergan, B., et al., European Respiratory Society guidelines on long-term home non-invasive ventilation for management of COPD. Eur Respir J, 2019. 54(3).

43. Agusti, A., et al., Treatable traits: toward precision medicine of chronic airway diseases. Eur Respir J, 2016. 47(2): p. 410-9.

44. MacDonald, M.I., et al., Cardiac dysfunction during exacerbations of chronic obstructive pulmonary disease. Lancet Respir Med, 2016. 4(2): p. 138-48.

45. Rutten, E.P.A., et al., Disturbed intestinal integrity in patients with COPD: effects of activities of daily living. Chest, 2014. 145(2): p. 245-252.

46. Andrianopoulos, V., et al., Exercise-induced oxygen desaturation in COPD patients without resting hypoxemia. Respir Physiol Neurobiol, 2014. 190: p. 40-6.

47. Budden, K.F., et al., Emerging pathogenic links between microbiota and the gut-lung axis. Nat Rev Microbiol, 2017. 15(1): p. 55-63.

48. Turner, J.R., Intestinal mucosal barrier function in health and disease. Nat Rev Immunol, 2009. 9(11): p. 799-809.

49. Shavelle, R.M., et al., Life expectancy and years of life lost in chronic obstructive pulmonary disease: findings from the NHANES III Follow-up Study. Int J Chron Obstruct Pulmon Dis, 2009. 4: p. 137-48.

50. Mannino, D.M., et al., Lung function and mortality in the United States: data from the First National Health and Nutrition Examination Survey follow up study. Thorax, 2003. 58(5): p. 388-93. 
51. Celli, B.R., et al., The body-mass index, airflow obstruction, dyspnea, and exercise capacity index in chronic obstructive pulmonary disease. N Engl J Med, 2004. 350(10): p. 1005-12.

52. Rodriguez-Roisin, R., et al., Ventilation-perfusion imbalance and chronic obstructive pulmonary disease staging severity. J Appl Physiol (1985), 2009. 106(6): p. 1902-8.

53. Augustin, I.M.L., et al., Comprehensive Lung Function Assessment Does not Allow to Infer Response to Pulmonary Rehabilitation in Patients with COPD. J Clin Med, 2018. 8(1).

54. Agusti, A., et al., Precision medicine in COPD exacerbations. Lancet Respir Med, 2018. 6(9): p. 657-659.

55. McCullough, P.A., et al., Uncovering heart failure in patients with a history of pulmonary disease: rationale for the early use of B-type natriuretic peptide in the emergency department. Acad Emerg Med, 2003. 10(3): p. 198-204.

56. Abroug, F., et al., Association of left-heart dysfunction with severe exacerbation of chronic obstructive pulmonary disease: diagnostic performance of cardiac biomarkers. Am J Respir Crit Care Med, 2006. 174(9): p. 990-6.

57. Couturaud, F., et al., Prevalence of Pulmonary Embolism Among Patients With COPD Hospitalized With Acutely Worsening Respiratory Symptoms. JAMA, 2021. 325(1): p. 59-68.

58. Celli, B.R., Dissecting COPD exacerbations: time to rethink our definition. Eur Respir J, 2017. 50(3).

59. Mannino, D.M., D.E. Doherty, and A. Sonia Buist, Global Initiative on Obstructive Lung Disease (GOLD) classification of lung disease and mortality: findings from the Atherosclerosis Risk in Communities (ARIC) study. Respir Med, 2006. 100(1): p. 115-22.

60. Pascoe, S., et al., Blood eosinophil counts, exacerbations, and response to the addition of inhaled fluticasone furoate to vilanterol in patients with chronic obstructive pulmonary disease: a secondary analysis of data from two parallel randomised controlled trials. Lancet Respir Med, 2015.

61. Rabe, K.F., et al., Triple Inhaled Therapy at Two Glucocorticoid Doses in Moderate-to-Very-Severe COPD. N Engl J Med, 2020. 383(1): p. 35-48.

62. Zhang, Q., et al., Risk of Pneumonia with Different Inhaled Corticosteroids in COPD Patients: A MetaAnalysis. COPD, 2020. 17(4): p. 462-469.

63. Vedel-Krogh, S., et al., Blood Eosinophils and Exacerbations in Chronic Obstructive Pulmonary Disease. The Copenhagen General Population Study. Am J Respir Crit Care Med, 2016. 193(9): p. 965-74.

64. Bafadhel, M., et al., Blood Eosinophils and Outcomes in Severe Hospitalized Exacerbations of COPD. Chest, 2016. 150(2): p. 320-8.

65. Gudmundsson, G., et al., Long-term survival in patients hospitalized for chronic obstructive pulmonary disease: a prospective observational study in the Nordic countries. Int J Chron Obstruct Pulmon Dis, 2012. 7: p. 571-6.

66. Ho, T.W., et al., In-hospital and one-year mortality and their predictors in patients hospitalized for firstever chronic obstructive pulmonary disease exacerbations: a nationwide population-based study. PLoS One, 2014. 9(12): p. e114866.

67. Singanayagam, A., S. Schembri, and J.D. Chalmers, Predictors of mortality in hospitalized adults with acute exacerbation of chronic obstructive pulmonary disease. Ann Am Thorac Soc, 2013. 10(2): p. 81-9.

68. Puhan, M.A., et al., Expansion of the prognostic assessment of patients with chronic obstructive pulmonary disease: the updated BODE index and the ADO index. Lancet, 2009. 374(9691): p. 704-11.

69. Feng, Z., et al., Efficacy of Various Scoring Systems for Predicting the 28-Day Survival Rate among Patients with Acute Exacerbation of Chronic Obstructive Pulmonary Disease Requiring Emergency Intensive Care. Can Respir J, 2017. 2017: p. 3063510.

70. Echevarria, C., et al., The PEARL score predicts 90-day readmission or death after hospitalisation for acute exacerbation of COPD. Thorax, 2017. 72(8): p. 686-693.

71. Chang, C.L., et al., Predicting early mortality in acute exacerbation of chronic obstructive pulmonary disease using the CURB65 score. Respirology, 2011. 16(1): p. 146-51.

72. Jones, S.E., et al., Sarcopenia in COPD: prevalence, clinical correlates and response to pulmonary rehabilitation. Thorax, 2015. 70(3): p. 213-8.

73. Chung, H.Y., et al., Redefining Chronic Inflammation in Aging and Age-Related Diseases: Proposal of the Senoinflammation Concept. Aging Dis, 2019. 10(2): p. 367-382.

74. Bruunsgaard, H., et al., Predicting death from tumour necrosis factor-alpha and interleukin-6 in 80-yearold people. Clin Exp Immunol, 2003. 132(1): p. 24-31. 
75. Gordon, C.J., et al., Serum biomarkers of aging in the Brown Norway rat. Exp Gerontol, 2011. 46(11): p. 953-7.

76. Agusti, A., et al., Persistent systemic inflammation is associated with poor clinical outcomes in COPD: a novel phenotype. PLoS One, 2012. 7(5): p. e37483.

77. Ito, K. and P.J. Barnes, COPD as a disease of accelerated lung aging. Chest, 2009. 135(1): p. 173-80.

78. Rutten, E.P., et al., Various Mechanistic Pathways Representing the Aging Process Are Altered in COPD. Chest, 2016. 149(1): p. 53-61.

79. Barnes, P.J., J. Baker, and L.E. Donnelly, Cellular Senescence as a Mechanism and Target in Chronic Lung Diseases. Am J Respir Crit Care Med, 2019.

80. Gan, W.Q., et al., Female smokers beyond the perimenopausal period are at increased risk of chronic obstructive pulmonary disease: a systematic review and meta-analysis. Respir Res, 2006. 7: p. 52.

81. Sorheim, I.C., et al., Gender differences in COPD: are women more susceptible to smoking effects than men? Thorax, 2010. 65(6): p. 480-5.

82. Vanfleteren, L.E., et al., Clusters of comorbidities based on validated objective measurements and systemic inflammation in patients with chronic obstructive pulmonary disease. Am J Respir Crit Care Med, 2013. 187(7): p. 728-35.

83. Prescott, E., et al., Gender difference in smoking effects on lung function and risk of hospitalization for COPD: results from a Danish longitudinal population study. Eur Respir J, 1997. 10(4): p. 822-7.

84. Lange, P., et al., Relation of ventilatory impairment and of chronic mucus hypersecretion to mortality from obstructive lung disease and from all causes. Thorax, 1990. 45(8): p. 579-85.

85. McGhan, R., et al., Predictors of rehospitalization and death after a severe exacerbation of COPD. Chest, 2007. 132(6): p. 1748-55.

86. Faustini, A., et al., The impact on risk-factor analysis of different mortality outcomes in COPD patients. Eur Respir J, 2008. 32(3): p. 629-36.

87. Dransfield, M.T., et al., Gender differences in the severity of CT emphysema in COPD. Chest, 2007. 132(2): p. 464-70.

88. Li, X., et al., Responsiveness to Ipratropium Bromide in Male and Female Patients with Mild to Moderate Chronic Obstructive Pulmonary Disease. EBioMedicine, 2017. 19: p. 139-145

89. Long term domiciliary oxygen therapy in chronic hypoxic cor pulmonale complicating chronic bronchitis and emphysema. Report of the Medical Research Council Working Party. Lancet, 1981. 1(8222): p. 6816.

90. Landbo, C., et al., Prognostic value of nutritional status in chronic obstructive pulmonary disease. Am J Respir Crit Care Med, 1999. 160(6): p. 1856-61.

91. Lainscak, M., et al., Body mass index and prognosis in patients hospitalized with acute exacerbation of chronic obstructive pulmonary disease. J Cachexia Sarcopenia Muscle, 2011. 2(2): p. 81-86.

92. Marott, J.L., et al., Lung Function Trajectories Leading to Chronic Obstructive Pulmonary Disease as Predictors of Exacerbations and Mortality. Am J Respir Crit Care Med, 2020. 202(2): p. 210-218.

93. Carter, R.I. and R.A. Stockley, Disease 'activity', 'severity' and 'impact': interrelationships in COPD; is a measure of disease 'activity' the Holy Grail for COPD, or a variable impossible to quantify? COPD, 2014. 11(4): p. 363-7.

94. Franssen, F.M.E. and L. Vanfleteren, Differential diagnosis and impact of cardiovascular comorbidities and pulmonary embolism during COPD exacerbations, in ERS Monograph: Acute Exacerbations of Pulmonary Diseases, P.R. Burgel, M. Contoli, and J.L. Lopez-Campos, Editors. 2017, European Respiratory Society: Sheffield. p. 114-128.

95. Foucher, P., et al., Relative survival analysis of 252 patients with COPD receiving long-term oxygen therapy. Chest, 1998. 113(6): p. 1580-7.

96. Seneff, M.G., et al., Hospital and 1-year survival of patients admitted to intensive care units with acute exacerbation of chronic obstructive pulmonary disease. JAMA, 1995. 274(23): p. 1852-7.

97. Echevarria, C., et al., Validation of the DECAF score to predict hospital mortality in acute exacerbations of COPD. Thorax, 2016. 71(2): p. 133-40.

98. Pauwels, R.A., et al., Global strategy for the diagnosis, management, and prevention of chronic obstructive pulmonary disease. NHLBI/WHO Global Initiative for Chronic Obstructive Lung Disease (GOLD) Workshop summary. Am J Respir Crit Care Med, 2001. 163(5): p. 1256-76. 
99. Augustin, I.M.L., et al., The respiratory physiome: Clustering based on a comprehensive lung function assessment in patients with COPD. PLoS One, 2018. 13(9): p. e0201593.

100. Criner, R.N., et al., Relationship between diffusion capacity and small airway abnormality in COPDGene. Respir Res, 2019. 20(1): p. 269.

101. Harvey, B.G., et al., Risk of COPD with obstruction in active smokers with normal spirometry and reduced diffusion capacity. Eur Respir J, 2015. 46(6): p. 1589-1597.

102. Connors, A.F., Jr., et al., Outcomes following acute exacerbation of severe chronic obstructive lung disease. The SUPPORT investigators (Study to Understand Prognoses and Preferences for Outcomes and Risks of Treatments). Am J Respir Crit Care Med, 1996. 154(4 Pt 1): p. 959-67.

103. Garcia-Aymerich, J., et al., Lung function impairment, COPD hospitalisations and subsequent mortality. Thorax, 2011. 66(7): p. 585-90.

104. Press, V.G., et al., Reducing Chronic Obstructive Pulmonary Disease Hospital Readmissions. An Official American Thoracic Society Workshop Report. Ann Am Thorac Soc, 2019. 16(2): p. 161-170.

105. Shah, T., et al., COPD Readmissions: Addressing COPD in the Era of Value-based Health Care. Chest, 2016. 150(4): p. 916-926.

106. Holguin, F., et al., Comorbidity and mortality in COPD-related hospitalizations in the United States, 1979 to 2001. Chest, 2005. 128(4): p. 2005-11.

107. Axson, E.L., et al., Hospitalisation and mortality in patients with comorbid COPD and heart failure: a systematic review and meta-analysis. Respir Res, 2020. 21(1): p. 54.

108. Blee, J., et al., Dispensing inhalers to patients with chronic obstructive pulmonary disease on hospital discharge: Effects on prescription filling and readmission. Am J Health Syst Pharm, 2015. 72(14): $p$ 1204-8.

109. Puhan, M.A., et al., Pulmonary rehabilitation following exacerbations of chronic obstructive pulmonary disease. Cochrane Database Syst Rev, 2016. 12: p. CD005305.

110. Vasilopoulou, M., et al., Home-based maintenance tele-rehabilitation reduces the risk for acute exacerbations of COPD, hospitalisations and emergency department visits. Eur Respir J, 2017. 49(5).

111. Vanfleteren, L.E., et al., Management of chronic obstructive pulmonary disease beyond the lungs. Lancet Respir Med, 2016.

112. Rabe, K.F., et al., Effect of roflumilast in patients with severe COPD and a history of hospitalisation. Eur Respir J, 2017. 50(1).

113. Bourbeau, J., et al., Reduction of hospital utilization in patients with chronic obstructive pulmonary disease: a disease-specific self-management intervention. Arch Intern Med, 2003. 163(5): p. 585-91.

114. Fan, V.S., et al., A comprehensive care management program to prevent chronic obstructive pulmonary disease hospitalizations: a randomized, controlled trial. Ann Intern Med, 2012. 156(10): p. 673-83.

115. Hartley, T., et al., The NIV Outcomes (NIVO) Score: prediction of in-hospital mortality in exacerbations of COPD requiring assisted ventilation. Eur Respir J, 2021

116. Knaus, W.A., et al., APACHE II: a severity of disease classification system. Crit Care Med, 1985. 13(10): p. 818-29.

117. Wildman, M.J., et al., A new measure of acute physiological derangement for patients with exacerbations of obstructive airways disease: the COPD and Asthma Physiology Score. Respir Med, 2007. 101(9): p. 1994-2002.

118. Lim, W.S., et al., Defining community acquired pneumonia severity on presentation to hospital: an international derivation and validation study. Thorax, 2003. 58(5): p. 377-82.

119. Duan, J., et al., Early prediction of noninvasive ventilation failure in COPD patients: derivation, internal validation, and external validation of a simple risk score. Ann Intensive Care, 2019. 9(1): p. 108.

120. Bott, J., et al., Randomised controlled trial of nasal ventilation in acute ventilatory failure due to chronic obstructive airways disease. Lancet, 1993. 341(8860): p. 1555-7.

121. Brochard, L., et al., Noninvasive ventilation for acute exacerbations of chronic obstructive pulmonary disease. N Engl J Med, 1995. 333(13): p. 817-22.

122. Struik, F.M., et al., Nocturnal non-invasive positive pressure ventilation for stable chronic obstructive pulmonary disease. Cochrane Database Syst Rev, 2013(6): p. CD002878. 
123. Kohnlein, T., et al., Non-invasive positive pressure ventilation for the treatment of severe stable chronic obstructive pulmonary disease: a prospective, multicentre, randomised, controlled clinical trial. Lancet Respir Med, 2014. 2(9): p. 698-705.

124. Murphy, P.B., et al., Effect of Home Noninvasive Ventilation With Oxygen Therapy vs Oxygen Therapy Alone on Hospital Readmission or Death After an Acute COPD Exacerbation: A Randomized Clinical Trial. JAMA, 2017. 317(21): p. 2177-2186.

125. Engelen, M.P., et al., Nutritional depletion in relation to respiratory and peripheral skeletal muscle function in out-patients with COPD. Eur Respir J, 1994. 7(10): p. 1793-7.

126. Vrieze, A., et al., Low bone mineral density in COPD patients related to worse lung function, low weight and decreased fat-free mass. Osteoporos Int, 2007. 18(9): p. 1197-202.

127. O'Hare, P.E., et al., Coronary artery calcification on computed tomography correlates with mortality in chronic obstructive pulmonary disease. J Comput Assist Tomogr, 2014. 38(5): p. 753-9.

128. Rutten, F.H., et al., Unrecognized heart failure in elderly patients with stable chronic obstructive pulmonary disease. Eur Heart J, 2005. 26(18): p. 1887-94.

129. Triest, F.J.J., et al., Disease-Specific Comorbidity Clusters in COPD and Accelerated Aging. J Clin Med, 2019. 8(4).

130. Rutten, E.P., et al., GI symptoms in patients with COPD. Chest, 2014. 145(6): p. 1437-8.

131. Enaud, R., et al., The Gut-Lung Axis in Health and Respiratory Diseases: A Place for Inter-Organ and Inter-Kingdom Crosstalks. Front Cell Infect Microbiol, 2020. 10: p. 9.

132. Zamarron, C., et al., Heart rate variability in patients with severe chronic obstructive pulmonary disease in a home care program. Technol Health Care, 2014. 22(1): p. 91-8.

133. Malo, O., et al., [Systemic inflammation during exacerbations of chronic obstructive pulmonary disease]. Arch Bronconeumol, 2002. 38(4): p. 172-6.

134. Wild, G.E., et al., The mechanisms of prednisone inhibition of inflammation in Crohn's disease involve changes in intestinal permeability, mucosal TNFalpha production and nuclear factor kappa B expression.

Aliment Pharmacol Ther, 2003. 18(3): p. 309-17.

135. Cazzola, M., et al., Beta2-agonist therapy in lung disease. Am J Respir Crit Care Med, 2013. 187(7): p. 690-6.

136. Grassi, G., Assessment of sympathetic cardiovascular drive in human hypertension: achievements and perspectives. Hypertension, 2009. 54(4): p. 690-7.

137. Haarmann, H., et al., Inhaled beta-agonist does not modify sympathetic activity in patients with COPD. BMC Pulm Med, 2015. 15: p. 46.

138. Sandek, A., et al., Altered intestinal function in patients with chronic heart failure. J Am Coll Cardiol, 2007. 50(16): p. 1561-9.

139. Chelakkot, C., J. Ghim, and S.H. Ryu, Mechanisms regulating intestinal barrier integrity and its pathological implications. Exp Mol Med, 2018. 50(8): p. 103.

140. Thomsen, M., et al., Inflammatory biomarkers and exacerbations in chronic obstructive pulmonary disease. JAMA, 2013. 309(22): p. 2353-61.

141. Oga, T., et al., Analysis of the relationship between health status and mortality in hypercapnic patients with noninvasive ventilation. Clin Respir J, 2017. 11(6): p. 772-780.

142. Duiverman, M.L., Noninvasive ventilation in stable hypercapnic COPD: what is the evidence? ERJ Open Res, 2018. 4(2).

143. Zantah, M., et al., The Mechanisms of Benefit of High-Flow Nasal Therapy in Stable COPD. J Clin Med, 2020. 9(12).

144. Storgaard, L.H., et al., Long-term effects of oxygen-enriched high-flow nasal cannula treatment in COPD patients with chronic hypoxemic respiratory failure. Int J Chron Obstruct Pulmon Dis, 2018. 13: p. 11951205.

145. Braunlich, J., et al., Nasal high-flow versus noninvasive ventilation in patients with chronic hypercapnic COPD. Int J Chron Obstruct Pulmon Dis, 2019. 14: p. 1411-1421.

146. Rea, H., et al., The clinical utility of long-term humidification therapy in chronic airway disease. Respir Med, 2010. 104(4): p. 525-33.

147. Pisani, L., et al., Extracorporeal CO2 removal (ECCO2R) in patients with stable COPD with chronic hypercapnia: a proof-of-concept study. Thorax, 2020. 75(10): p. 897-900. 


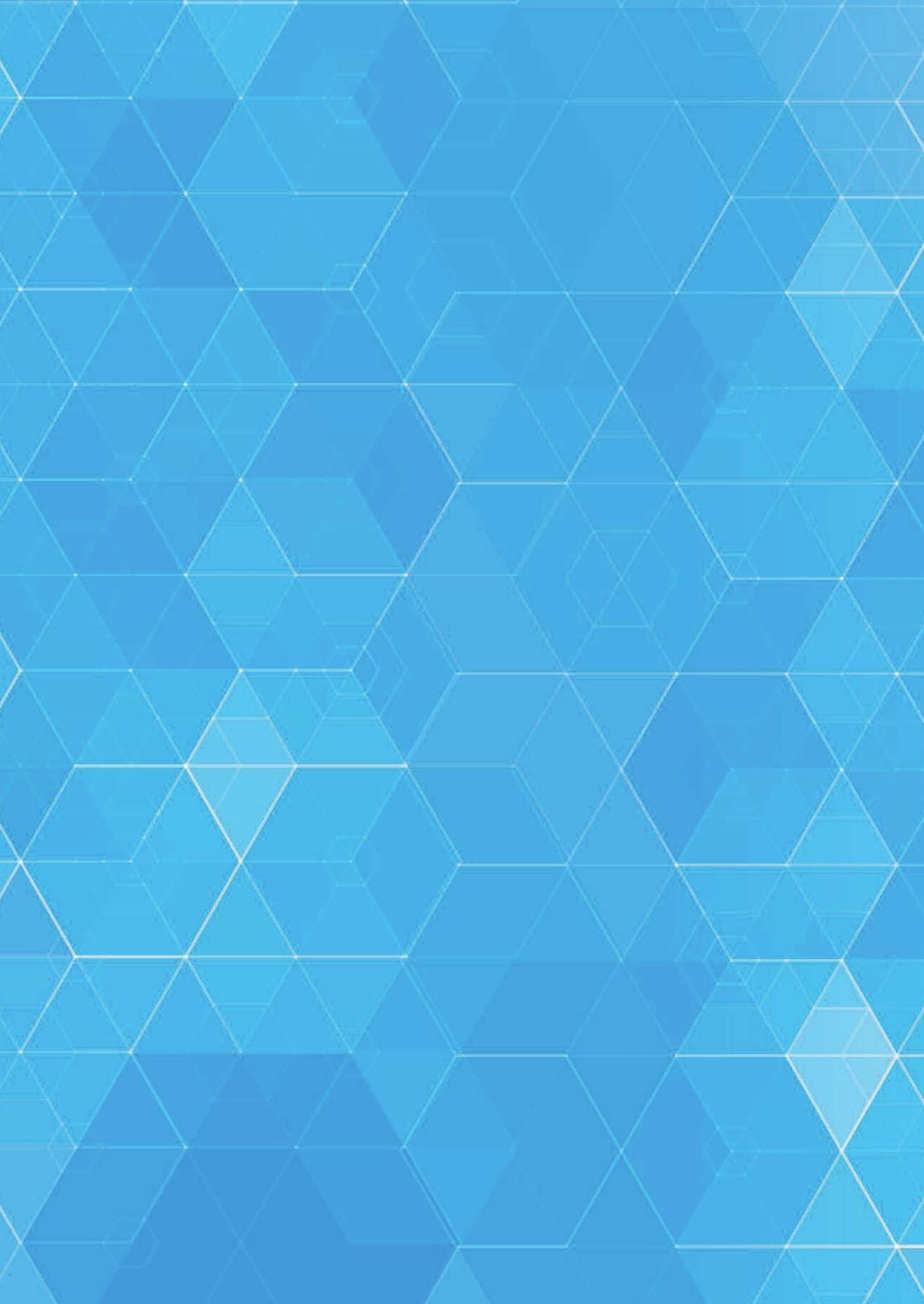




\section{Summary}





\section{Summary}

Chronic obstructive pulmonary disease (COPD) is characterized by persistent respiratory symptoms and airflow limitation, resulting from complex interactions between genetic susceptibility, lung development and environmental exposures. Worldwide, COPD is a highly prevalent disease with numbers expected to rise tremendously in the coming decades due to population growth and ageing. In 2017, COPD represented more than half of chronic respiratory disease prevalence and accounted for significant morbidity and mortality. In 2020 the Global Burden of Disease study reported that COPD was ranked as the fourth leading cause of death of the world in persons between $\mathbf{5 0 - 7 5}$ years, after ischemic heart disease, stroke and diabetes.

In addition to a variety of daily symptoms including dyspnea, fatigue, cough and sputum, the clinical course of COPD may be complicated by the occurrence of exacerbations of the disease. These events are defined by acute worsening of clinical symptoms resulting in additional therapies. Respiratory infections play an important role in the pathophysiology of exacerbations. Exacerbations vary in severity, ranging from events treated with rescue inhaler therapies at home to events requiring hospitalization for acute respiratory failure. These hospitalizations represent a pivotal healthcare problem in the Netherlands as direct costs of COPD in the Netherlands are for more than $55 \%$ driven by emergency visits and hospitalizations related to exacerbations. Also, they are associated with disease progression and substantial mortality risk.

The presentation of COPD exacerbations (ECOPD) in the hospital is heterogeneous, regarding patient characteristics, underlying triggers for exacerbation and the degree and nature of respiratory failure. Also, comorbid conditions contribute to the symptomatic deterioration in individual patients, while the impact of the exacerbation per se on other organs, including gut homeostasis, is largely unexplored. Thus, it remains difficult to predict short- and longer-term outcomes in individual patients. Especially, management of patients with acute life-threatening hypercapnic respiratory failure remains challenging, as prognostic indicators are less studied in this very severe subgroup and treatment options for preventing recurrence and lowering mortality risk are limited.

This thesis aimed to investigate the short- and long-term mortality rates for COPD patients hospitalized for severe exacerbations with or without respiratory failure and to study predictors for poor outcomes. Also, investigating the effects of non-invasive 
ventilation (NIV) in the post-acute phase of severe exacerbation and studying gastrointestinal (GI) integrity in relation to exacerbations of COPD were goals of this thesis.

In chapter 3 long-term survival following hospitalization for severe exacerbation was studied in a large population of COPD patients and compared with a gender- and agematched general population. The 5, 10 and 15 years survival in this COPD group was $43.7 \%, 19.9 \%$ and $7.3 \%$, respectively. In the general population survival was $76.9 \%$, $57.2 \%$ and $40.6 \%$ respectively. The results confirm that patients hospitalized for exacerbation have poor prognosis. The 15 years survival categorized by GOLD stage I-IV was $24 \%, 11.1 \%, 5.3 \%$ and $0 \%$, respectively. The mean life expectancy for these patients per GOLD stage I-IV was estimated at 9.7, 7.1, 6.1 and 3.4 years, whereas the life expectancy in the health matched controls was 10.2 years. Several independent prognostic factors were identified, including advanced age, male gender, low $\mathrm{FEV}_{1}$, low TLCO, respiratory failure, the presence of comorbidities, ICU admission during index admission and exacerbation frequency.

The study presented in chapter 4 reported on 1-year prognosis following hospitalized exacerbation of COPD in a different patient population. An in-hospital mortality rate of $5.8 \%$ and a 1 -year mortality of $27.7 \%$ were observed. Furthermore, six clinically available and strong independent predictors for 1 year mortality at hospital admission for ECOPD were identified: age, male gender, previous hospitalization for exacerbation, congestive heart failure in medical history, hypercapnia and elevated urea at admission. Next to the high mortality rate during 1-year the follow-up, another important aspect was the high readmission rate. Of those surviving the hospital admission for ECOPD more than half (55.1\%) had been re-hospitalized during follow up.

Developing an easy-to-use model to identify patients who are at the greatest risk for death during or shortly after hospitalization will be helpful in clinical daily practice, will facilitate shared decision making and eventually might improve disease management. The use of CART analysis to construct such a model or risk stratification could be a useful novel alternative approach. In the study in chapter 5 almost $10 \%$ of patient did not survive the hospital admission for ECOPD and $16.2 \%$ died within 90 days after admission. Based on analysis of an extensive dataset collected at time of admission, four independent predictors for 90-days mortality were identified in the CART: advanced age, hypercapnia, low body mass index and previous admission for ECOPD in the last 2 years. The data showed that if patients with ECOPD were admitted accompanied with these four positive factors, mortality risk was $50 \%$. In absence of any of these factors, none of the hospitalized ECOPD patients died within 90 days follow-up. 
Chapter 6 focussed on COPD patients presenting with acute respiratory failure and requiring NIV for the first time in their disease trajectory. In this retrospective cohort study, in-hospital, 1-year and 2-year mortality rates of $14.1 \%, 43.6 \%$ and $56.4 \%$ respectively, indicating that these patients constitute a very severe subgroup. Independent predictors for poor prognosis were NIV usage longer than 8 days, older age, and non-successful respond to NIV.

In chapter 7 a randomized controlled study investigating the effectiveness of nocturnal non-invasive ventilation in patients with COPD with prolonged hypercapnia after ventilatory support for acute respiratory failure is described. Patients underwent randomisation to NIV or standard care and were followed for 1 year. Time to readmission for respiratory causes or death were comparable in both groups, although NIV improved day- and night-time carbon dioxide levels.

In the second part of this thesis interaction between exacerbations and functional integrity of the gastro-intestinal (GI) tract were explored. The presented study in chapter $\mathbf{8}$ was the first to demonstrate increased small intestinal permeability during severe exacerbation complicated by hypoxemic respiratory failure compared to a following clinical stable condition.

In chapter 9, it was reported that GI integrity is decreased in COPD compared to controls. No difference in GI integrity was observed between COPD patients with or without frequent exacerbations or hospitalizations. Furthermore, baseline GI integrity was not a predictive of subsequent exacerbations.

In chapter $\mathbf{1 0}$ the results of the thesis were discussed and put in a broader perspective and directions for further studies were provided.

In conclusion, this thesis confirmed the detrimental impact of severe exacerbations on prognosis of COPD patients and identified several risk factors for poor outcome on short- and longer-term. Also, it contributed to our understanding of the role of NIV in the management of patients with persistent hypercapnia during hospitalization and it provided insights in disturbances in gastro-intestinal integrity in relation to exacerbations of COPD. 


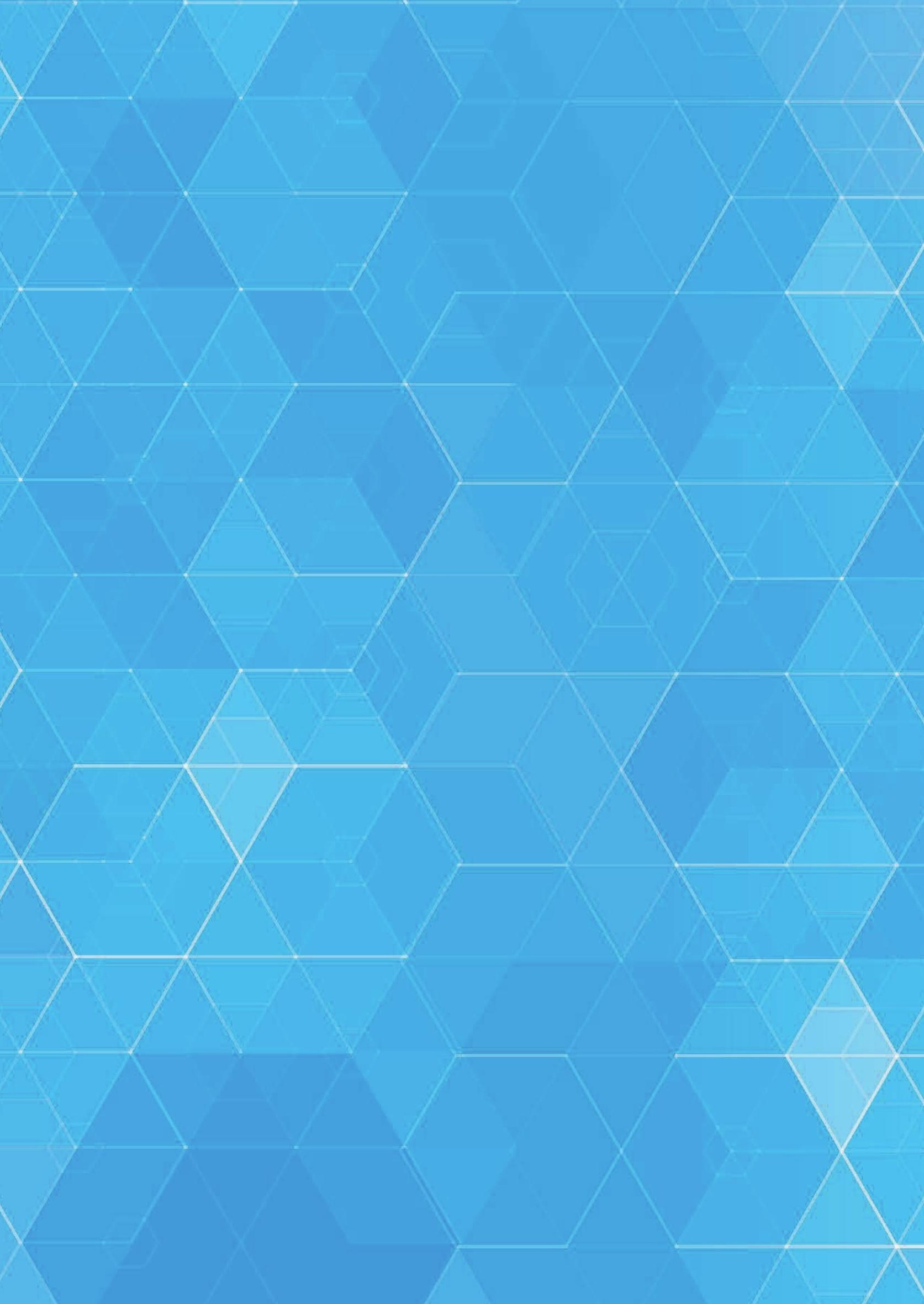




\section{Samenvatting}





\section{Samenvatting}

Chronische obstructieve Longaandoening (COPD) wordt gekenmerkt door aanhoudende longklachten en luchtwegobstructie als gevolg van complexe interacties tussen genetische gevoeligheid, longontwikkeling en blootstelling aan het milieu. Wereldwijd is COPD een veel voorkomende ziekte waarvan het aantal naar verwachting de komende decennia enorm zal stijgen als gevolg van bevolkingsgroei en vergrijzing. In 2017 vertegenwoordigde COPD meer dan de helft van de prevalentie van chronische luchtwegaandoeningen en was dit verantwoordelijk voor significante morbiditeit en mortaliteit. In 2020 meldde de Global Burden of Disease-studie dat COPD werd gerangschikt als de vierde belangrijkste doodsoorzaak ter wereld bij personen tussen 50-75 jaar, na ischemische hartziekte, beroerte en diabetes.

Naast een verscheidenheid aan dagelijkse symptomen, waaronder kortademigheid, vermoeidheid, hoesten en sputum, kan het klinische beloop van COPD gecompliceerd worden door het optreden van exacerbaties van de ziekte. Deze gebeurtenissen worden gedefinieerd door een acute verslechtering van klinische symptomen, resulterend in aanvullende therapieën. Luchtweginfecties spelen een belangrijke rol bij de pathofysiologie van exacerbaties. Exacerbaties variëren in ernst, variërend van voorvallen die thuis worden behandeld met rescue-inhalatietherapieën tot voorvallen waarvoor ziekenhuisopname vereist is voor acuut respiratoir falen. Deze ziekenhuisopnames vormen een cruciaal gezondheidsprobleem in Nederland, aangezien de directe kosten van COPD in Nederland voor meer dan 55\% worden veroorzaakt door spoedbezoeken en ziekenhuisopnames in verband met exacerbaties. Ze worden ook in verband gebracht met ziekteprogressie en een aanzienlijk sterfterisico.

De presentatie van COPD-exacerbaties (ECOPD) in het ziekenhuis is heterogeen, wat betreft patiëntkenmerken, onderliggende triggers voor exacerbatie en de aard en de ernst van respiratoir falen. Ook dragen co-morbiditeiten bij aan de symptomatische verslechtering bij individuele patiënten, terwijl de impact van de exacerbatie op zich op andere organen, waaronder de homeostase van de darmen, grotendeels nog onontgonnen is. Het blijft dus moeilijk om uitkomsten op korte en langere termijn bij individuele patiënten te voorspellen. Vooral de behandeling van patiënten met acuut levensbedreigend hypercapnisch respiratoir falen tijdens exacerbatie COPD blijft een uitdaging. Met name aangezien prognostische indicatoren minder bestudeerd zijn in deze zeer ernstige subgroep en behandelingsopties, om herhaling te voorkomen en het sterfterisico te verlagen, beperkt zijn. 
Dit proefschrift had tot doel het sterftecijfer op korte en lange termijn te onderzoeken van COPD patiënten die in het ziekenhuis zijn opgenomen voor ernstige exacerbaties met of zonder respiratoir falen, en om voorspellers voor slechte uitkomsten te bestuderen. Ook het onderzoeken van de effecten van niet-invasieve beademing (NIV) in de post-acute fase van ernstige exacerbatie en het bestuderen van de gastrointestinale (GI) integriteit in relatie tot exacerbaties van COPD waren doelstellingen van dit proefschrift.

In hoofdstuk 3 werd de overleving op de lange termijn na ziekenhuisopname voor ernstige exacerbaties bestudeerd in een grote populatie van COPD-patiënten en vergeleken met een algemene populatie van hetzelfde geslacht en leeftijd. De 5, 10 en 15 jaar overleving in deze COPD-groep was respectievelijk 43,7\%, 19,9\% en 7,3\%. In de algemene populatie was de overleving respectievelijk $76,9 \%, 57,2 \%$ en $40,6 \%$. De resultaten bevestigen dat patiënten die wegens exacerbatie in het ziekenhuis zijn opgenomen, een slechte prognose hebben. De overleving na 15 jaar gecategoriseerd volgens GOLD stadium I-IV was respectievelijk 24\%, 11,1\%, 5,3\% en 0\%. De gemiddelde levensverwachting voor deze patiënten per GOLD-stadium I-IV werd geschat op 9,7, 7,1, 6,1 en 3,4 jaar, terwijl de levensverwachting in de gezonde gematchte controles 10,2 jaar was. Verschillende onafhankelijke prognostische factoren werden

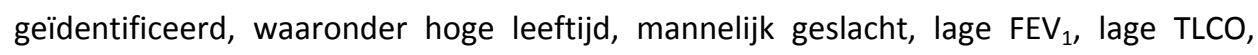
respiratoire insufficiëntie, de aanwezigheid van co-morbiditeit, IC-opname tijdens indexopname en exacerbatiefrequentie.

De studie gepresenteerd in hoofdstuk 4 rapporteerde over een prognose van 1 jaar na exacerbatie van COPD in het ziekenhuis bij een andere patiëntenpopulatie. Er werd een sterftecijfer in het ziekenhuis van 5,8\% en een 1-jaars sterfte van $27,7 \%$ waargenomen. Verder werden zes klinisch beschikbare en sterke onafhankelijke voorspellers voor 1 jaar mortaliteit bij ziekenhuisopname voor ECOPD geïdentificeerd: leeftijd, mannelijk geslacht, eerdere ziekenhuisopname wegens exacerbatie, congestief hartfalen in de medische geschiedenis, hypercapnie en verhoogd ureum bij opname. Naast het hoge sterftecijfer tijdens de follow-up van 1 jaar, waren de hoge heropnamen een ander belangrijk aspect. Van degenen die de ziekenhuisopname voor ECOPD overleefden, was meer dan de helft $(55,1 \%)$ opnieuw in het ziekenhuis opgenomen tijdens de follow-up.

Het ontwikkelen van een gebruiksvriendelijk model om patiënten te identificeren, die het grootste risico lopen op overlijden tijdens of kort na ziekenhuisopname, zal nuttig zijn in de dagelijkse klinische praktijk, zal de gedeelde besluitvorming vergemakkelijken en zou uiteindelijk het ziektebeheer kunnen verbeteren. Het gebruik van CART-analyse 
om een dergelijk model of risicostratificatie te construeren, zou een bruikbare nieuwe alternatieve benadering kunnen zijn. In de studie in hoofdstuk 5 overleefde bijna $10 \%$ van de patiënten de ziekenhuisopname voor ECOPD niet en stierf 16,2\% binnen 90 dagen na opname. Op basis van analyse van een uitgebreide dataset verzameld op het moment van opname, werden vier onafhankelijke voorspellers voor 90-dagen mortaliteit geïdentificeerd in de CART: gevorderde leeftijd, hypercapnie, lage body mass index en eerdere opname voor ECOPD in de afgelopen 2 jaar. De gegevens toonden aan dat als patiënten met ECOPD werden opgenomen in combinatie met deze vier positieve factoren, het sterfterisico $50 \%$ was. Bij afwezigheid van een van deze factoren stierf geen van de in het ziekenhuis opgenomen patiënten met een exacerbatie COPD binnen 90 dagen follow-up.

Hoofdstuk 6 concentreerde zich op COPD-patiënten met acuut respiratoir falen en die voor het eerst NIV nodig hadden in hun ziektetraject. In deze retrospectieve cohortstudie waren de sterftecijfers in het ziekenhuis, 1 jaar en 2 jaar respectievelijk $14,1 \%, 43,6 \%$ en $56,4 \%$, hetgeen aangeeft dat deze patiënten een zeer ernstige subgroep vormen. Onafhankelijke voorspellers voor een slechte prognose waren NIVgebruik langer dan 8 dagen, oudere leeftijd en niet-succesvolle respons op NIV.

In hoofdstuk 7 wordt een gerandomiseerde gecontroleerde studie beschreven waarin de effectiviteit van nachtelijke niet-invasieve beademing wordt onderzocht bij patiënten met COPD met langdurige hypercapnie na beademingsondersteuning voor acuut respiratoir falen. Patiënten ondergingen randomisatie naar NIV of standaardzorg en werden gedurende 1 jaar gevolgd. De tijd tot heropname wegens respiratoire oorzaken of overlijden was vergelijkbaar in beide groepen, hoewel de NIV de kooldioxidegehaltes overdag en 's nachts verbeterde.

In het tweede deel van dit proefschrift werd de interactie tussen exacerbaties en functionele integriteit van het maagdarmkanaal (GI) onderzocht. De gepresenteerde studie in hoofdstuk 8 was de eerste die een verhoogde permeabiliteit van de dunne darm aantoonde tijdens ernstige exacerbaties, gecompliceerd door hypoxemisch respiratoir falen in vergelijking met een later volgende klinische stabiele toestand.

In hoofdstuk 9 werd gerapporteerd dat de integriteit van het maagdarmkanaal verminderd is bij COPD in vergelijking met controles. Er werd geen verschil in GIintegriteit waargenomen tussen COPD-patiënten met of zonder frequente exacerbaties of ziekenhuisopnames. Bovendien was baseline Gl-integriteit geen voorspellende waarde voor latere exacerbaties. 
In hoofdstuk 10 werden de resultaten van dit proefschrift bediscussieerd en in een breder perspectief geplaatst en werden aanwijzingen gegeven voor verder onderzoek.

Concluderend bevestigde dit proefschrift de enorme negatieve impact van ernstige exacerbaties op de prognose van COPD patiënten en identificeerde verschillende risicofactoren voor een slechte uitkomst op korte en langere termijn. Het proefschrift droeg ook bij tot ons begrip van de rol van NIV bij de behandeling van patiënten met aanhoudende hypercapnie na een ziekenhuisopname en het proefschrift leverde nieuwe inzichten over de stoornissen in de gastro-intestinale integriteit in relatie tot exacerbaties van COPD. 



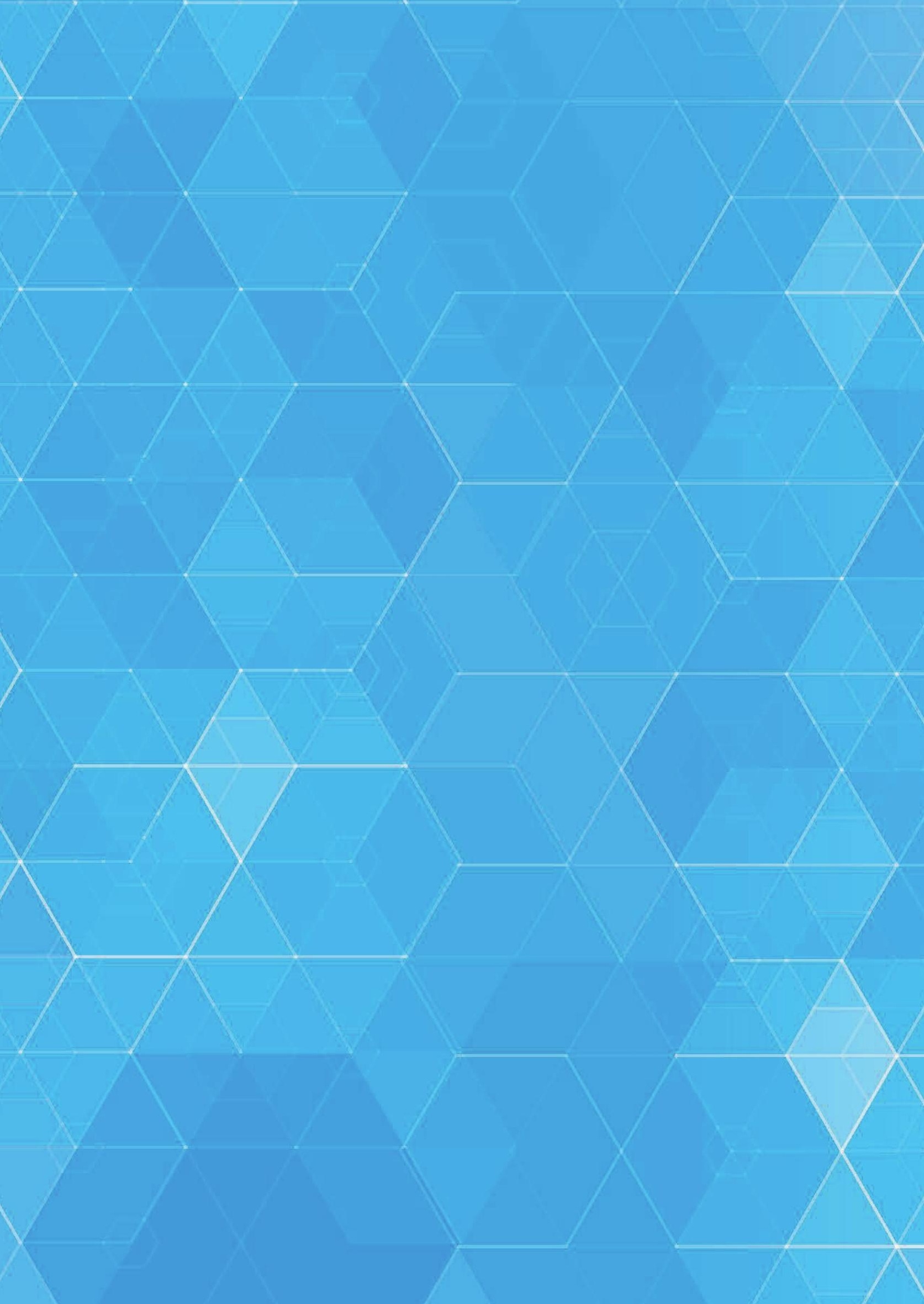




\section{Impact paragraph}





\section{Impact paragraph}

The present chapter describes the scientific and social impact of this thesis. Otherwise, a description of the impact of the created value from the gathered knowledge by making it suitable for and/or available in clinical practice and translating that information into practical services, products or tools. First, the aims and main findings of the thesis are briefly described. Next, the scientific and public relevance and its impact on clinical practice are discussed. Thereafter, target groups of this thesis are mentioned. Finally, activities and opportunities will be discussed.

\section{Aims and main findings of this thesis}

Severe exacerbations of chronic obstructive pulmonary disease (COPD) have a detrimental impact on patients with this disease and impose a great burden on healthcare systems and societies. In order to improve management and outcomes of these events, this thesis aimed to investigate short- and longer-term mortality rates for COPD patients hospitalized for exacerbations with or without acute respiratory failure. The second aim of the thesis was to identify predictors for poor outcomes and to analyze the effects of non-invasive ventilation (NIV) in the acute and post-acute phase of severe exacerbations. Finally, the thesis investigated gastro-intestinal permeability in relation to exacerbations, as alterations in gut barrier function might be causally related to these events.

In four chapters of this thesis, high mortality rates following hospitalization for COPD exacerbation were shown in different patient populations. These vary from approximately $6-14 \%$ for inpatient mortality, to $16-30 \%$ after three months, $28-44 \%$ after one year and $>70 \%$ after more than ten years of follow up. The thesis shows that the hospitalized COPD population is heterogeneous regarding disease severity and need for medical management, which probably accounts for the variation in mortality rates between different chapters.

Several independent predictors for mortality following hospitalized COPD exacerbations were identified in this thesis, including age, male gender, poor lung function, increased arterial carbon dioxide levels, previous exacerbations, co-occurring diseases and low body weight. A risk stratification tool for 90-day mortality was developed in this thesis and included four of these factors. 
The effectiveness of long-term nocturnal NIV in patients with COPD with prolonged hypercapnia after ventilatory support for acute respiratory failure was investigated in this thesis and was negative regarding its primary endpoints: time to readmission for respiratory causes or death.

Increased small intestine permeability was observed COPD patients hospitalized with acute hypoxemic respiratory failure, while in another chapter, reduced gut integrity was observed in stable COPD patients in comparison to controls, with no association with the occurrence of exacerbations.

\section{Relevance}

The results regarding the impact of severe exacerbations on in-hospital, short- and long-term mortality and their predictors are highly relevant for the scientific community. They raise awareness among scientists and enforce the need for research in this field of COPD to study interventions to modify the risk factors for poor outcomes and reduce mortality. Possible traits that were identified in this thesis and that could be accessible for intervention include (accelerated) aging, low body weight, increased arterial carbon dioxide, comorbid conditions and poor lung function.

The negative results regarding the early initiation of NIV following ventilatory support, contributed to the better understanding of the target population for NIV and the development of subsequent intervention studies by other scientific groups in this field. Ultimately, this has resulted in the implementation of NIV in the management of severe COPD with chronic respiratory failure.

The observations regarding alterations in gut function in COPD patients and the association with exacerbations help to increase scientific awareness regarding the systemic consequences of COPD and the need for a holistic approach if scientists aim to unravel the complex pathophysiology of COPD and discover targets for innovative, potentially disease-modifying, interventions.

\section{Target groups}

In addition to the scientific community, the main target group of this thesis are the clinicians such as the pulmonologists, intensive care physicians and general 
practitioners, who are involved in the management of the COPD patients. Other health care providers who are involved in caring for COPD patients like physician assistants, nurse practitioners and respiratory nurses are also a target audience. For these health care workers, the results of this thesis point out the impact of severe exacerbations and the need to optimize patient management in order to reduce this impact. Also, the results of this thesis provide them with knowledge regarding treatable patient characteristics if they aim to reduce mortality risk. With the risk stratification tool provided in this thesis, clinicians have the opportunity to assess the adverse outcome on admission and this tool may support shared decision making and advance care planning leading to personalized medicine. Also, the results of this thesis highlight that patients with at first sight mild or moderate COPD may develop acute respiratory failure and need for NIV.

Pharmaceutical companies might be interested in the potentially modifiable traits associated with mortality in severe exacerbations in their search for targets for novel medications. This also applies to organizations involved in the development of nonpharmacological interventions.

From an educational point of view the results of the thesis are also relevant. For students and trainees, they provide important information about the tremendous impact of COPD on mortality and its determinants, the positioning of NIV and some of the systemic aspects of the disease.

Moreover, the results of this thesis are relevant to patients and their informal caregivers. They emphasize the importance of preventing severe exacerbations and give them insight in the predictors of poor outcomes. Also, they demonstrate to patients that COPD is more than a respiratory disease, demanding a more holistic approach.

Furthermore, the reported detrimental impact of severe exacerbations in this thesis is relevant for health care policy makers and payers and could result in prioritization of COPD management and reinforcement of health care resources and development of innovative programmes to change this trend. 


\section{Activities}

The demonstrated results of the thesis have led to several activities in this domain of research and field of expertise. The findings of chapters $\mathbf{3}$ to $\mathbf{9}$ were transformed into original manuscripts and published or submitted in different scientific international journals. Moreover, several experts in the field wrote editorial letters to highlight on these manuscripts, especially to the manuscripts of chapter $\mathbf{4 , 5}$ and 7. Furthermore, the results presented in chapters $\mathbf{3}$ to $\mathbf{9}$ were presented during the annual European Respiratory Society (ERS) congresses in 2013 (Barcelona, Spain), 2014 (Munich, Germany), 2016 (London, United Kingdom), 2017 (Milan, Italy) and 2020 (virtual, Vienna, Austria). The knowledge presented in this thesis is also used in daily clinical practice at the department of Respiratory Medicine in Maastricht UMC+ and included in the education of trainees during the round table visits and of medical and other students at Faculty of Health, Medicine and Life Sciences of Maastricht University. As a consequence of these activities, the findings of the thesis have been distributed broadly and hopefully this thesis will support clinicians identifying new clinically-related questions, inspire researchers for innovative studies and raise awareness among policy makers and payers regarding the personal and societal burden and subsequent needs associated with severe COPD exacerbations. 



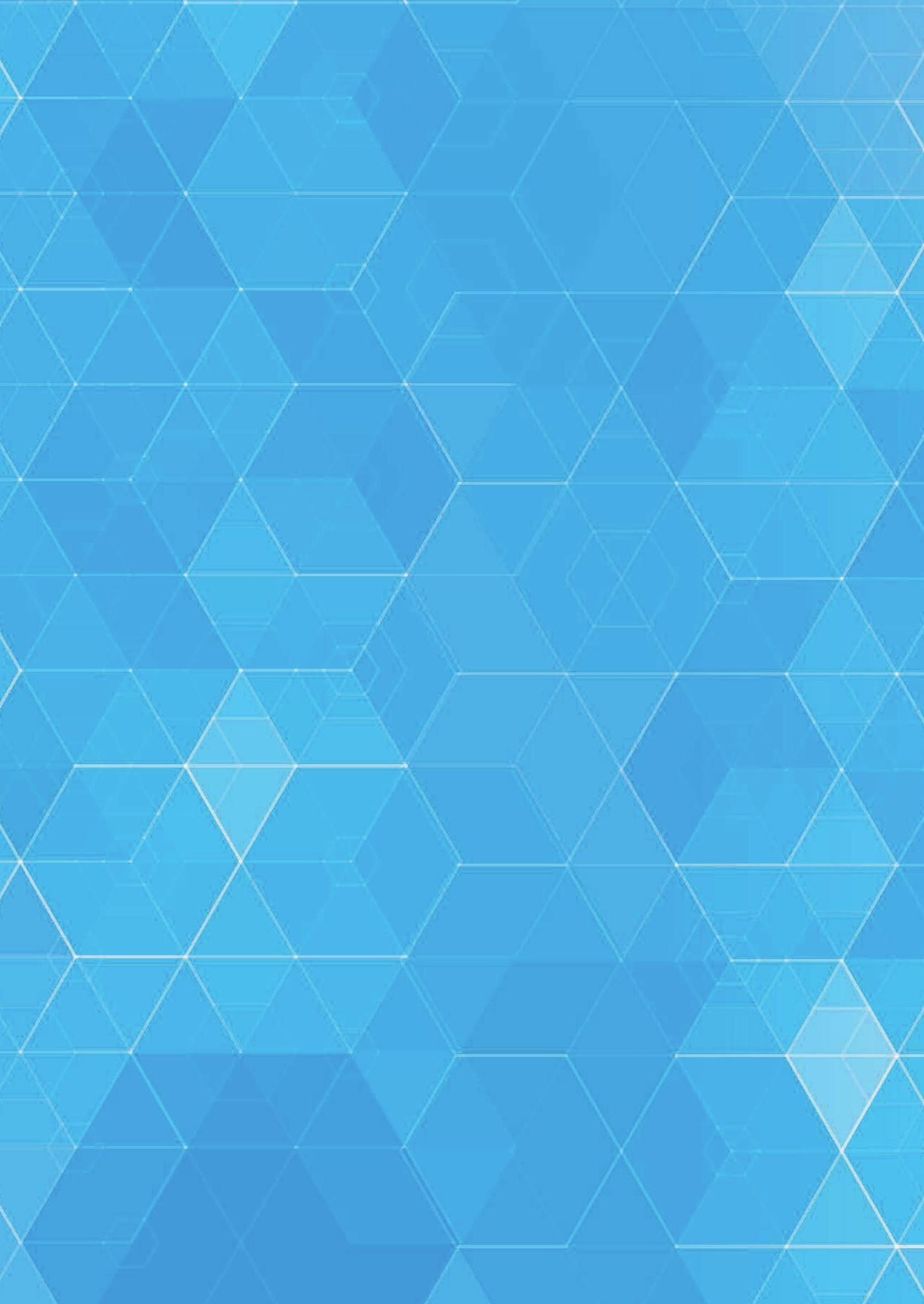





\section{Dankwoord}

Het is gelukt! De aanhouder wint! Een proefschrift maken doe je niet alleen!

Zodoende is dit hoofdstuk van "het Boekje" een zeer belangrijk hoofdstuk. Het biedt mij met veel plezier de gelegenheid om iedereen te bedanken die, op welke wijze dan ook, heeft bijgedragen aan het tot stand komen van dit proefschrift.

Graag wil ik mijn promotor professor dr. Wouters bedanken voor zijn coaching, reflectie, kritische blik en feedback tijdens het proces om te komen tot dit proefschrift. Tevens ben ik professor dr. Wouters zeer erkentelijk voor het in mij gestelde vertrouwen en voor zijn geduld met mij en mijn onderzoek. Mede doorslaggevend voor mij om dit project op te pakken, waren de door hem geboden mogelijkheden. Bij deze wil ik graag ook mijn bewondering uitspreken over zijn planmatige en visionaire begeleiding tijdens mijn promotietraject. Met name de momenten waarop ik uitgedaagd werd om de verdieping te zoeken, waren voor mij waardevolle "eyeopeners". Ik ben er erg trots op deel te mogen zijn van de "Wouters promovendi family". Mijn oprechte dank hiervoor!

(Co)promotoren: professor dr. Gernot Rohde en professor dr. Frits Franssen.

Beste Gernot, beste Frits, bedankt voor jullie intense en prettige begeleiding. Zonder jullie hulp was dit alles niet gelukt!

Gernot, je hebt mij geholpen om dit project aan te zwengelen. Heel veel dank hiervoor. Jouw tomeloze inzet om mij te helpen vergeet ik zeker niet. Ook jouw motto, "ask for forgiveness not for permission", heb ik omarmd. Helaas kon je de Duitse aanbieding van de Goethe kliniek niet weerstaan. Maar ook op afstand hield je een oogje in het zeil. In de korte tijd dat we samenwerkten bij de afdeling longziekten van het MUMC+ is er een hechte vriendschap ontstaan.

Frits, jij kwam later in dit spel. Maar het klikte meteen tussen jou en mij, als longartsen in het MUMC+, maar ook als team tijdens dit promotietraject. Je brede blik, je nauwgezette werkwijze en grote betrokkenheid bij dit project, hebben op een zeer positieve en prettige wijze bijgedragen aan mijn persoonlijke ontwikkeling en last but not least aan dit proefschrift. Tevens was je de waakhond die mij gefocust hield. Je manier van werken, stap voor stap, is voor mij een voorbeeld. 
Beste heren, nogmaals wil ik mijn respect en bewondering uitspreken over jullie wetenschappelijke ambitie, jullie enthousiasme en schrijfkunsten. Voor mij waren jullie mijn baken en inspiratiebron!

Geertjan Wesseling, Monique Hochstenbag, Nicolle Cobben, Lennart Conemans, Sami Simons, Marijke Rutten, Janna Schoenmaekers, Lizza Hendriks, Rein Posthuma, Bettine Vosse, Kim Walraven en Safiye Dursun: mijn geweldige collega's longartsen. Jullie wil ik graag bedanken voor jullie steun en oprechte interesse in mij en dit onderzoek. Daarnaast bewonder ik het begrip en het vertrouwen in elkaar. Dit leidt tot een hechte groep met een constructieve en plezierige werksfeer.

Natuurlijk mag ik de verpleegkundigen van de afdeling Longziekten niet vergeten te bedanken.

Veel werk werd mij ook uit handen genomen door mijn collega's van het secretariaat Longziekten. Met name Marjo van Tol was zeer waardevol bij het organiseren en plannen van mijn agenda en afspraken.

Ook een speciaal woord van dank voor mijn collega's van het Centrum voor Thuisbeademing Maastricht. Jullie oprechte interesse in mij en dit onderzoek waardeer ik zeer. Ik heb bewondering voor jullie enthousiasme, geduld en commitment in verband met de zorg van onze patiënten.

Ook de "WESP studenten" (Roel, Ilvy, Evelien en Wouter) wil ik graag bedanken voor hun ondersteuning bij de voorbereiding van onderzoek en invoer van de studieresultaten.

Oud collega Astrid Otte, jou wil ik bedanken voor jouw raad en daad in barre tijden. Helaas ben je naar het Zuyderland ziekenhuis vertrokken. Maar onze respectvolle vriendschap blijft.

Kortom betere collega's kan een mens zich niet wensen!

Beste co-auteurs, jullie zijn met teveel om allemaal op te noemen. Bedankt voor jullie inhoudelijke, kritische feedback en waardevolle aanvullingen op de artikelen. Elk artikel is het resultaat van een gezamenlijke inspanning.

Dear co-authors, thank for your critical appraisal and valuable input on the articles. Each article we achieved together.

De leden van de beoordelingscommissie dank ik voor de grondige bestudering, kritische beoordeling en goedkeuring van dit proefschrift. Naast de voorzitter professor dr. Richard Koopmans bestond de beoordelingscommissie uit professor dr. Harry Crijns, professor dr. Wim Janssens (Katholieke Universiteit Leuven, België), professor dr. Johan Verbraecken (Universiteit Antwerpen, België) en Dr. Dennis Bergmans. 
Dear professor dr. Richard Koopmans (chair), professor dr. Harry Crijns, professor dr. Wim Janssens (Katholieke Universiteit Leuven, België), professor dr. Johan Verbraecken (Universitair Ziekenhuis Antwerpen, België), Dr. Dennis Bergmans thank you for your critical assessment and approval of this thesis.

De "fietsbreurs" Jules, Pascal en Roger. Tijdens deze promotie en full time longarts kon de boog niet altijd gespannen blijven. De fiets-uitjes/-rondes zorgden voor een goede balans van spanning en ontspanning en zijn dus van onschatbare waarde geweest. "Breurs" dank hiervoor!

Beste Rob, onze gewaardeerde en hechte vriendschap dateert al vanaf onze jeugdtijd. We steunen elkaar al jaren door dik en dun. Ook tijdens dit promotietraject was jij mijn maatje. Onze gezamenlijke "voetbalavonden" hielpen zeker mee om de balans tussen werk en ontspanning te bewaren!

Het "Rieleks" studenten groepje Niels, Diederd en Gian-Pierro, samen hebben we geweldige avonden, waarbij we allerlei ethische en maatschappelijke dilemma's bediscussiëren. Het liefst bij een heerlijk diner in een Maastrichts restaurant, waarbij dan ook nog oude herinneringen naar boven komen drijven.

Kortom beste vrienden, dankjewel voor de gezellige avonden en leuke feestjes zij zorgden voor mijn boordnodige ontspanning!

Lieve schoonfamilie, het zal voor jullie vaak een raadsel zijn geweest waar ik de afgelopen jaren mee bezig hield. Jullie support, gastvrijheid en flexibiliteit zijn lovenswaardig. Dank voor de het warme bad en interesse en gezelligheid en ik hoop nog heel lang van jullie levensplezier te kunnen genieten. Met name mijn schoonouders Wiel en Philomène, jullie staan altijd paraat met een positief woord en zorgen in ons drukke gezin voor rust en regelmaat. Heel veel dank daarvoor en ik bewonder het feit dat jullie altijd voor mij en mijn gezin klaar staan.

Dank wil ik ten zeerste uitspreken voor mijn vader die mij inspireerde en stimuleerde om te gaan studeren. Helaas werd hij ernstig ziek en stierf jong, als succesvol beeldhouwer. Zijn tomeloze inzet en zijn vastberadenheid om zijn inspiraties en zijn kunst te delen met de maatschappij vond ik bewonderingswaardig. De illustratie "Gebroken leven" op de voorzijde van dit proefschrift heb ik aan hem te danken. Het was voor mij een treffend symbool om de impact van ziekenhuisopname bij ernstige longaanvallen weer te geven. Van mijn vader heb ik de "ideeën bron" geërfd. Dit is van onschatbare waarde geweest bij de totstandkoming van de ideeën voor dit promotietraject. Hij stimuleerde mij ook om uitdagingen niet uit de weg te gaan. 
Mijn moeder gaf mij altijd goede raad, stond altijd voor me klaar en samen hebben we veel plezier beleefd. We dronken vaak koffie samen om mooie herinneringen op te halen en om moeilijke dilemma's te overzien. Ook was ze voor mijn kinderen een hele fijne oma. Helaas is het alweer bijna 3 jaar geleden dat een groot bloedig CVA haar plots noodlottig werd. Ik mis haar elke dag en dat zal eeuwig duren. Maar Mamma, met veel plezier denk ik vooral terug aan onze mooie herinneringen en jouw wijze raad.

Kortom lieve papa en mama, zonder mij ooit in bepaalde richting te hebben gedwongen, weet ik dat jullie erg trots zouden zijn op mij.

Dank spreek ik ook uit naar mijn zus Jolande (Jopie) en mijn schoonbroer Ben in Enschede. Ondanks de verre afstand waren jullie toch dichtbij. Bedankt voor jullie interesse in mijn wel en wee.

Ook mijn oudste zus Ivette (lefke) en mijn schoonbroer en speciale vriend Hans verdienen een bijzonder woord van dank.

Hans, jij bent al heel lang mijn schoonbroer en beste vriend. Je hebt mij sinds mijn jeugd geholpen bij het maken van belangrijke keuzes op mijn levenspad. Ik heb bewondering voor jouw reflecterend vermogen. De kritische maar doch respectvolle manier waarop je mijn gedachten en keuzes hebt ondersteund, waren voor mij een inspirerende leidraad. Het bood me de gelegenheid om met een heldere kijk mezelf te ontwikkelen en eigen beslissingen te nemen. Onze ontspannende momenten, waarop we als klus team optreden, waren vaak hilarisch en zorgden voor een vrolijke noot en ontspanning.

Ivette, jij was mijn partner in crime, jij geloofde altijd in mijn mogelijkheden en gaf mij het gevoel een bijzonder mens te zijn. Ook als het effe tegen zat, bleef je mij stimuleren en onvoorwaardelijk steunen. "De aanhouder wint" is ons gezamenlijk adagium! Je onuitputtelijke hulp en steun waren goud waard bij mijn medische studie. Met name de vele sessies Engelse bijles door jou tijdens mijn VWO-jaren waren onontbeerlijk. Je open-minded view, bevlogenheid en rijke levenservaring geven kleur aan mijn persoonlijke kijk op het leven. Bij het samenstellen van dit dankwoord en bij de keuze van de voorblad illustratie, heb ik dankbaar gebruik gemaakt van jouw bijzonder gevoel voor taal en creativiteit.

Tot slot ons hechte gezin. Onze kinderen Lars en Esmé. Ze schenken mij elke dag veel liefde en vreugde en ze zijn ongelofelijk belangrijk voor mij. Zij houden mij vaker een spiegel voor, soms confronterend, maar meestal verhelderend en vaak vergezeld met goede raad en hulp: goud waard en inspiratiebron. Ons gezin bestaat ook uit Teuntje, onze brave hond, die ons tijdens de Corona crisis in beweging houdt. 
Tenslotte wil ik mijn allerliefste en meest dierbare van iedereen, Inge mijn vrouw bedanken. Woorden schieten te kort. We kennen elkaar al heel lang. Je stralende lach in "den Ingel", zomer 1988, vergeet ik nooit. Je bent en blijft mijn eerste liefde. Zonder jou stond ik hier niet. Je onvoorwaardelijke steun, liefde en geloof in mij waren voor mij van onschatbare waarde en tevens een drive om samen gelukkig te zijn. Door de hectische baan als longarts in MUMC+, met onregelmatige werktijden en een promotie traject, was er soms minder tijd voor jou en mijn gezin. Desondanks maar vooral dankzij jou bleef alles in balans. De laatste tijd werd er vaker gevraagd wanneer is "het boekje" af. Er leek nooit een einde te komen aan mijn promotieonderzoek. Maar nu ben ik ECHT klaar met dit traject. Nogmaals heel veel dank voor je onvoorwaardelijke liefde, steun en het geloof in mij.

Ik draag dit proefschrift met trots op aan mijn ouders Marcel Sprooten en Leny van Loo. 


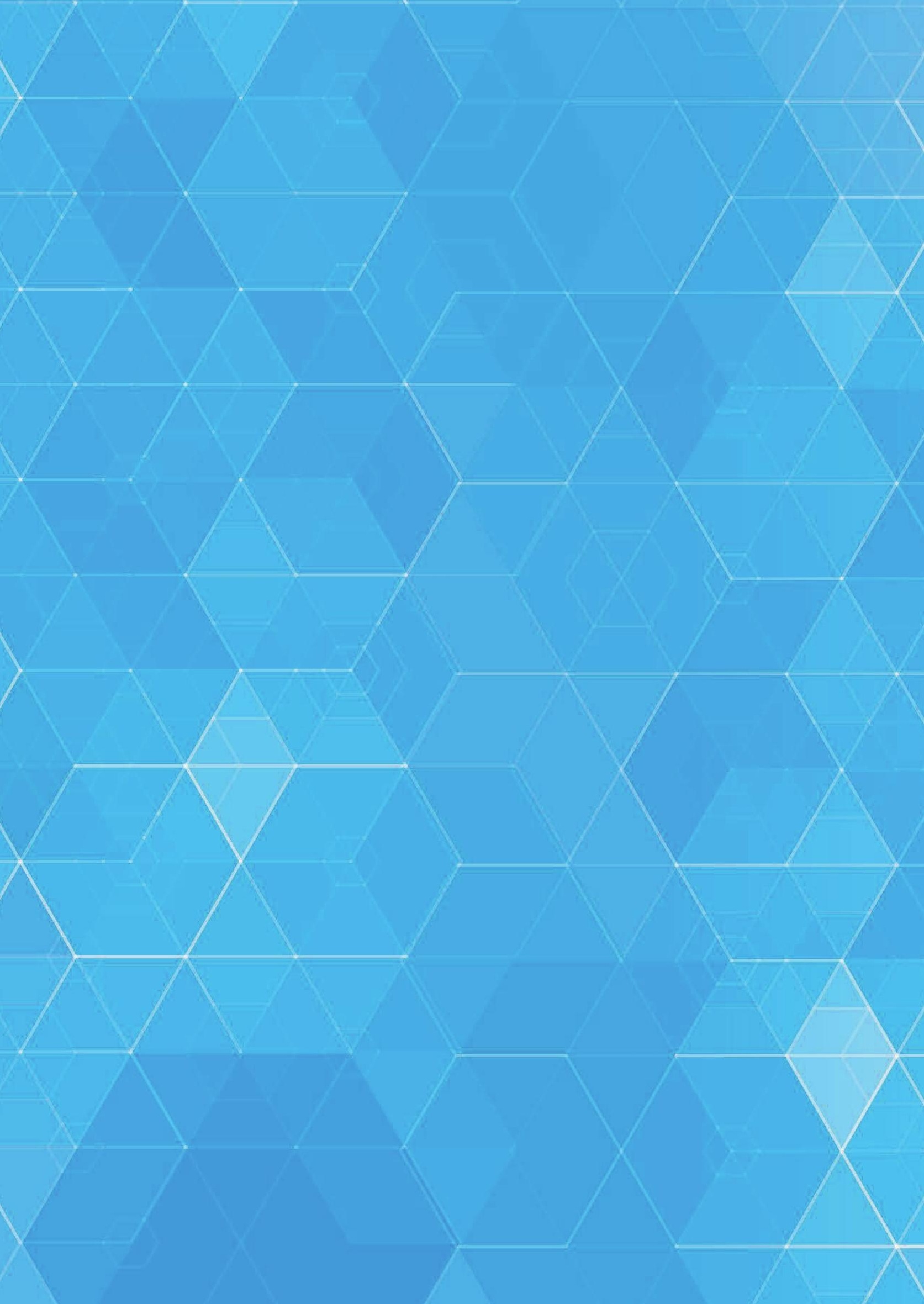




\section{Curriculum Vitae}





\section{Curriculum Vitae}

Roy (roepnaam) Theodora Marcel werd geboren op 18 september 1970 in Maastricht. In 1991 behaalde hij zijn VWO diploma aan het Trichter College in Maastricht. Dat zelfde jaar kon hij aan zijn felbegeerde opleiding Geneeskunde aan de Rijksuniversiteit Limburg beginnen. In 1997 rondde hij hier de studie geneeskunde af. Hierna werkte hij als arts assistent niet in opleiding bij interne geneeskunde en longziekten in verschillende zieken-huizen en uiteindelijk in het De Wever ziekenhuis (nu Zuyderland) in Heerlen bij de maatschap Longziekten "Oude Mijnstreek" alwaar hem een opleidingsplaats voor het specialisme Longziekten en Tuberculose werd aangeboden. Na 6 jaar noeste arbeid en plezier werd dit succesvol afgerond.

Sinds 2007 is Roy werkzaam als algemeen longarts en als thuisbeademingsarts bij de afdeling Longziekten te MUMC+ en het Centrum voor Thuisbeademing Maastricht (CtbM). Naast het klinische werk is hij nauw betrokken bij het onderwijs in het MUMC+. Hij geeft onderwijs, onder andere aan de geneeskunde faculteit in zowel het reguliere programma als in het internationale programma. Daarnaast verzorgt hij trainingen voor zorgprofessionals zoals bronchoscopie simulator training, cursus echografie thorax en de multidisciplinaire training in acute en chronische non-invasieve beademing. Naast deze taken is Roy sinds 2016 medisch coördinator van de Behandelkamer Longziekten en sinds 2019 chef de Polikliniek Longzieken. Namens de vakgroep Longziekten is hij lid van het Stafconvent in MUMC+, lid van de Trombosecommissie en lid van de Commissie Medische Technologie. Hij is lid van de Nederlandse Vereniging voor Artsen voor Longziekten en Tuberculose (NVALT) (Lid van secties COPD en pulmonale interventies) en European Respiratory Society (ERS). Verder is er een nauwe samenwerking met het Expertisecentrum voor Chronisch Orgaanfalen (CIRO), waarbij hij lid van de projectgroep Chronisch Respiratoir Falen bij COPD.

Eén van zijn ambities is, middels chronische beademing meer kwaliteit van leven aan patiënten met chronisch respiratoir falen te bieden. Zijn wetenschappelijk onderzoek is vooral gericht op onderzoek bij patiënten met (chronisch) respiratoir falen, met daarbij speciale aandacht voor diafragma dysfunctie en COPD. Verder heeft ook klinisch onderzoek naar COPD hospitalisaties en non invasieve beademing grote aandacht van Roy. Hiernaast bestaat een grote interesse voor endobronchiale interventies zoals de endobrochiale echografie (EBUS) en starre bronchoscopie met optie tot debulking.

Roy is een echte teamplayer die streeft naar collegialiteit, van elkaar leren en gebruik maken van elkaars expertise. Deze aspecten zijn belangrijke randvoorwaarden om samen te streven naar de best mogelijke zorg voor de "longpatient". 


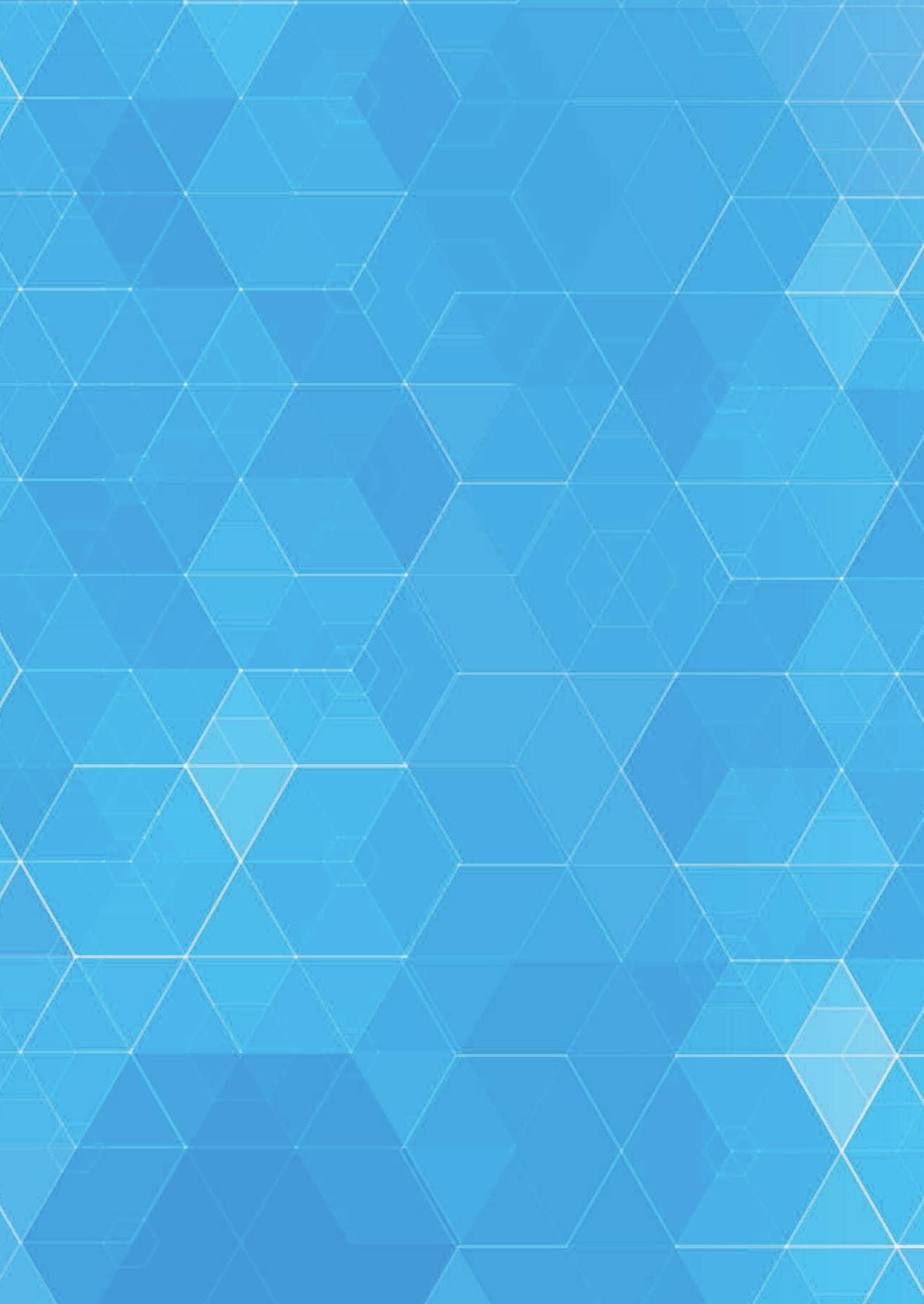




\section{List of publications}





\section{List of publications}

Predictors for long-term mortality in COPD patients requiring non-invasive positive pressure ventilation for the treatment of acute respiratory failure.

Sprooten R.T.M., Rohde G.G.U., Janssen M.T.H.F., Cobben N.A.M., E.F.M. Wouters, Franssen F.M.E.

Clinical Respiratory Journal; December 2020; Volume 14; Issue 12; Pages 1144-1152.

Mechanical ventilation variability due to tracheal compression in a patient with a mycotic descending aortic aneurysm: inverted relation to blood pressure.

Heuts S., Sprooten R.T.M., Heines S.J.H., Mees B.M.E.

European Journal of Cardio-Thoracic Surgery; October 2020; Volume 58; Issue 4; Pages 864-866.

Risk stratification for short-term mortality at hospital admission for acute exacerbation of COPD.

Sprooten R.T.M.*, Rohde G.G.U.*, Lawyer G., Leijte W.T., Wouters E.FM., Franssen F.M.E.

Respirology. August 2019; Volume 224, Issue 8; Pages 765-776.

*Equal authorship

Surgical treatment of bronchial rupture in blunt chest trauma: a review of literature. van Roozendaal L.M., van Gool M.H., Sprooten R.T.M., Maesen B.A.E., Poeze M., Hulsewé K.W.E., Vissers Y.L.J., de Loos E.R.

Journal of Thoracic Disease; September 2018; Volume 10; Issue 9; Pages 5576-5583.

Phrenic neuropathy and diaphragm dysfunction in neuralgic amyotrophic.

van Alfen N., Doorduin J., van Rosmalen M.H.J., van Eijk J.J.J., Heijdra Y., Boon A.J., Gaytant M.A., van den Biggelaar R.J.M., Sprooten R.T.M., Wijkstra P.J., Groothuis J.T. Neurology; August 2018; Volume 91; Issue 9; Pages e843-e849.

Increased small intestinal permeability during severe acute exacerbations of COPD.

Sprooten R.T.M., Lenaerts K., Braeken D.C.W., Grimbergen I., Rutten E.P., Wouters E.F.M., Rohde GGU.

Respiration; May 2018; Volume 95; Issue 5; Pages 334-342. 
Long term survival after admission for COPD exacerbation: a comparison with the general population.

van Hirtum P.V.*, Sprooten R.T.M.*, van Noord J.A., van Vliet M., de Kruif M.D.

Respiratory Medicine. April 2018; Volume 137; Pages 77-82.

*Equal authorship

Increased exercise tolerance using daytime mouthpiece ventilation for patients with diaphragm paralysis.

Koopman M., Vanfleteren L.E.G.W., Steijns S., Wouters E.F.M., Sprooten R.T.M.

Breathe; September 2017; Volume 13; Issue 3; Pages 225-229.

Case report: Bilateral diaphragmatic dysfunction due to Borrelia Burgdorferi.

Basunaid S., van der Grinten C., Cobben N., Otte A., Sprooten R.T.M., Gernot R.

F1000Research. October 2014; Volume 6; Issue 3; Page 235.

Nocturnal non-invasive ventilation in COPD patients with prolonged hypercapnia after ventilatory support for acute respiratory failure: a randomized, controlled, parallel-group study.

Struik F.M., Sprooten R.T.M., Kerstjens H.A., Bladder G., Zijnen M., Asin J., Cobben N.A., Vonk J.M., Wijkstra P.J.

Thorax; September 2014; Volume 69; Issue 9; Pages 826-34.

The Severe Respiratory Insufficiency Questionnaire scored best in the assessment of health-related quality of life in chronic obstructive pulmonary disease.

Struik F.M., Kerstjens H.A., Bladder G., Sprooten R.T.M., Zijnen M., Asin J., van der Molen T., Wijkstra P.J.

Journal of Clinical Epidemiology; October 2013; Volume 66; Issue 10; Pages 1166-74.

Predictors of 1-year mortality at hospital admission for acute exacerbations of chronic pulmonary disease.

Slenter R.H.*, Sprooten R.T.M.*, Kotz D., Wesseling G., Wouters E.F., Rohde G.G.

Respiration; December 2013; Volume 85 Issue 1; Pages 15-26.

*equal authorship

An adolescent with chest pain

Vleeshouwers G., Sprooten R.T.M., Rohde G.U.

Breath; 2013 volume 9: pages 309-316 


\section{Groente imiteert bronchuscarcinoom}

Vanfleteren L., Abdul-Hamid M., Sprooten R.T.M.

Tijdschrift voor Geneeskunde en Gezondheidszorg; 2010 volume 8: pages 374-6. 


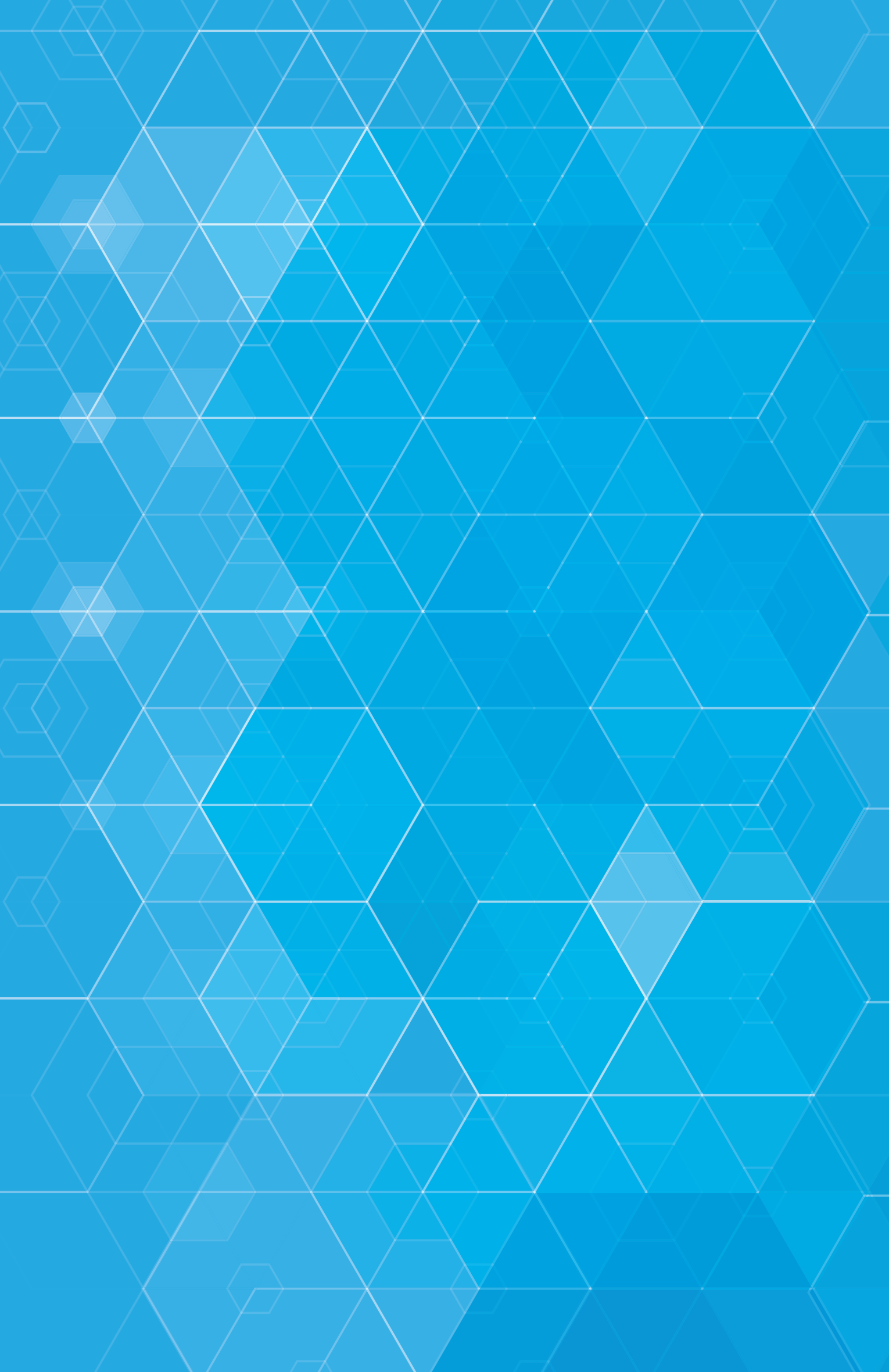

